NISTIR 8273

\title{
NIST Fatty Acid Quality Assurance Program 2017 Final Report
}

Bruce A. Benner, Jr. Jacolin A. Murray

This publication is available free of charge from:

https://doi.org/10.6028/NIST.IR.8273
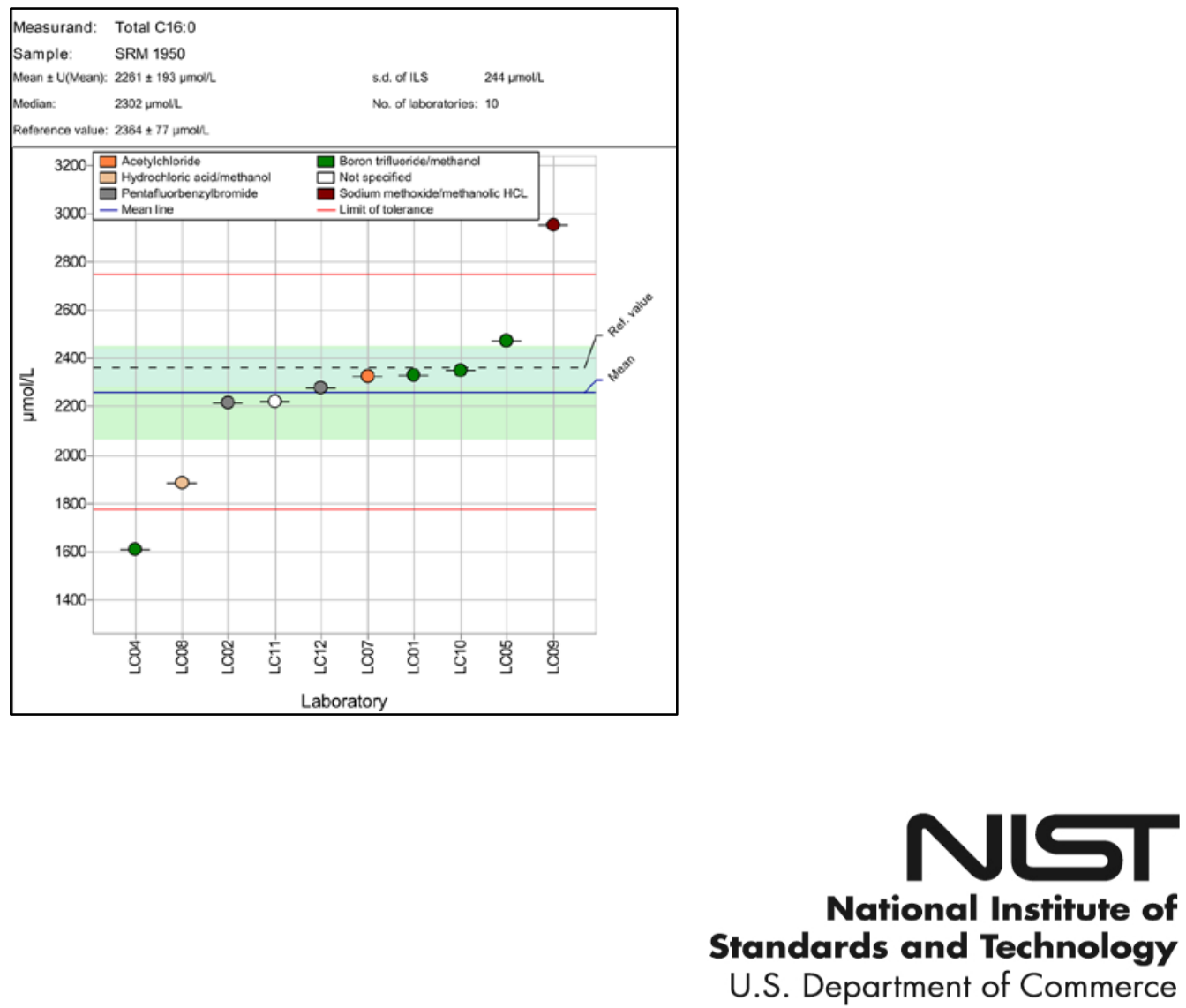
This page intentionally blank. 
NISTIR 8273

\title{
NIST Fatty Acid Quality Assurance Program 2017 Final Report
}

\author{
Bruce A. Benner, Jr. \\ Jacolin A. Murray \\ Chemical Sciences Division \\ Material Measurement Laboratory
}

This publication is available free of charge from:

https://doi.org/10.6028/NIST.IR.8273

October 2019

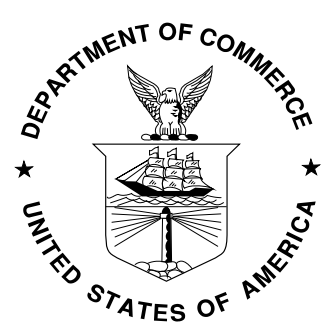

U.S. Department of Commerce

Wilbur L. Ross, Jr., Secretary

National Institute of Standards and Technology Walter Copan, NIST Director and Undersecretary of Commerce for Standards and Technology 
Certain commercial entities, equipment, or materials may be identified in this document in order to describe an experimental procedure or concept adequately. Such identification is not intended to imply recommendation or endorsement by the National Institute of Standards and Technology, nor is it intended to imply that the entities, materials, or equipment are necessarily the best available for the purpose.

National Institute of Standards and Technology Interagency or Internal Report 8273

Natl. Inst. Stand. Technol. Interag. Intern. Rep. 8273, 124 pages (October 2019)

This publication is available free of charge from: https://doi.org/10.6028/NIST.IR.8273 


\begin{abstract}
At the request of the National Institutes of Health (NIH) Office of Dietary Supplements (ODS) and in conjunction with the Centers for Disease Control and Prevention (CDC), in 2017 the National Institute of Standards and Technology (NIST) conducted the fourth Fatty Acid Quality Assurance Program (FAQAP) interlaboratory study of fatty acid (FA) concentrations in human serum. This 2017 FAQAP study included measurements of free FAs (extraction and measurement prior to acid/base hydrolysis) and total FAs (measurement after acid/base hydrolysis). Participants were requested to analyze SRM 1950 Metabolites in Frozen Human Plasma as a control, three "unknown" sera, and a free fatty acid (FFA) solution. Participants measuring fatty acid methyl ester (FAME) derivatives of FAs were asked to analyze a FAME solution. The results from this fourth exercise are reported along with a summary of the analytical methods used by the 14 participating laboratories.
\end{abstract}

\title{
Keywords
}

Fatty Acids (FAs), Free Fatty Acids (FFAs), Fatty Acid Methyl Esters (FAMEs) Human Serum, Interlaboratory Comparison Study, SRM 1950 


\section{Table of Contents}

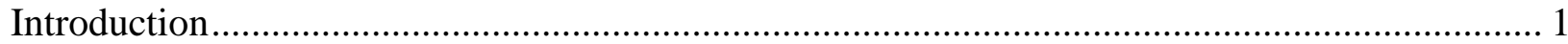

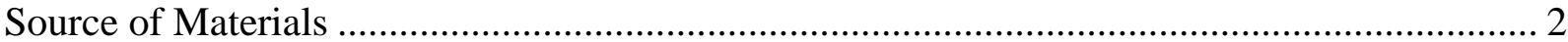

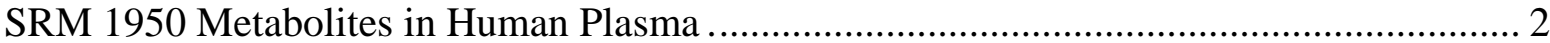

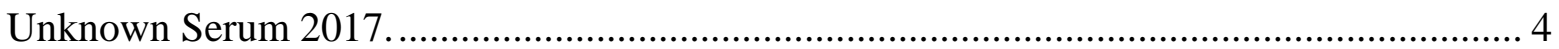

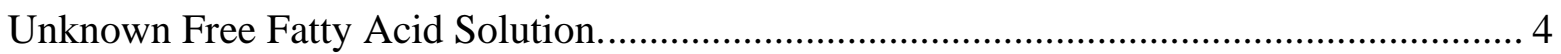

Unknown Fatty Acid/Fatty Acid Methyl Ester Solution....................................................... 5

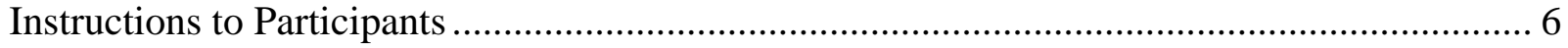

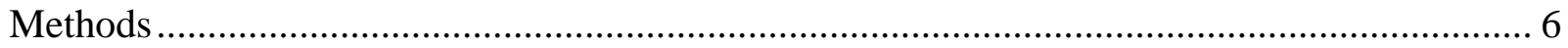

Overview of Data Treatment and Representation.................................................................... 9

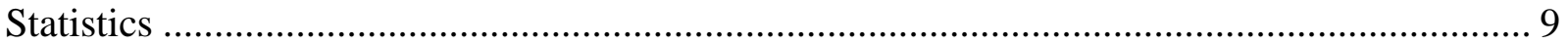

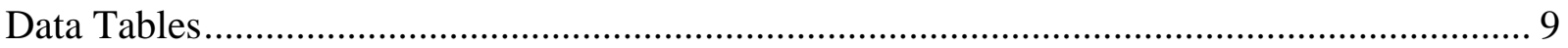

Graphical Representation: ............................................................................................. 9

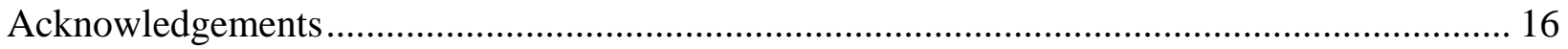

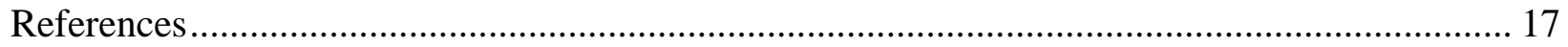

Appendix A: Free and Total Fatty Acids in Plasma and Serum ................................................... 18

Appendix B: Free Fatty Acids and Fatty Acid Methyl Esters in Solutions ................................... 58

Appendix C: Free Glycerol and Total Glycerides in 2017 Unknown Serum ................................ 87

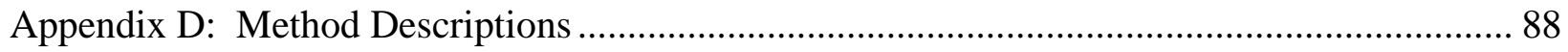

\section{List of Tables}

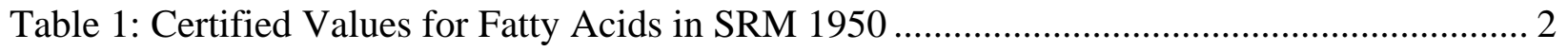

Table 2: Reference Values for Fatty Acids in SRM 1950 ............................................................ 3

Table 3: Gravimetric Concentration of Free Fatty Acids in the Unknown FFA Solution............... 4

Table 4: Fatty Acids and Fatty Acid Methyl Esters in the Unknown FA/FAME Solution............ 5

Table 5: Summary of Participant's Methods for Total Fatty Acids in Serum................................. 7

Table 6: Summary of Participant's Methods for Free Fatty Acids in Serum. .................................. 7

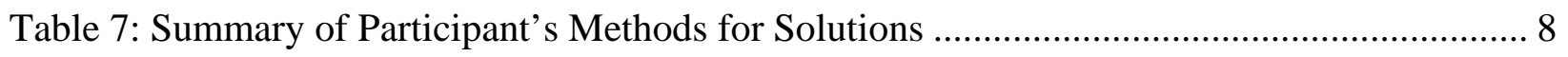

Table 8: Summary of Participant's Methods for Total Glycerides in Serum. ................................. 8

Table 9: Summary of Participant Method for Free Glycerol in Serum ........................................... 8

Table 10: Comparison of Consensus Mean and Range to SRM 1950 Total FA Values ............... 11

Table 11: Total Fatty Acids in Unknown Serum ...................................................................... 13

Table 12: Consensus Mean of Select Free Fatty Acids in SRM 1950 And Unknown Serum...... 14

Table 13: Comparison of Consensus Ranges to Gravimetric Target in Unknown FFA Solution 15

Table 14: Consensus Mean of atty Acid Methyl Esters in FAME Solution D............................ 16 


\section{Introduction}

The fatty acid (FA) content of human plasma and serum can serve as indicators of health status and diet quality. Additionally, levels of FAs measured in their free form in serum or plasma, prior to any acid or base hydrolysis, versus conjugated as triglycerides and phospholipids, have been proposed as possible health markers for metabolic, ${ }^{1}$ neurological, ${ }^{2}$ and cardiovascular $^{3},{ }^{4}$ diseases The National Institute of Standards and Technology (NIST) has offered the Fatty Acid Quality Assurance Program (FAQAP) through the NIST Clinical Quality Assurance Program (ClinQAP) since 2012. The first exercise of FAQAP was conducted in 2012 with 11 participants returning data for FAs in serum and plasma samples. ${ }^{5}$ The second exercise of the FAQAP was conducted in 2015 with 14 participants returning data for FAs in serum samples. ${ }^{6}$ The third exercise was conducted in 2016 with 13 participants reporting results for serum and plasma samples. ${ }^{7}$

This report summarizes the results from the 14 participants of the 2017 FAQAP. In this study, participants were asked to measure free and total FAs in three human sera samples (labeled as Unknown Serum 2017 A, B, and C, all three were the same serum material), SRM 1950 Metabolites in Frozen Human Plasma (control material), three free fatty acid solutions (labeled as Unknown Free FA Solution A, B, and C, all three were the same solution), and a fatty acid (FA)/fatty acid methyl ester (FAME) solution (labeled Unknown FA/FAME Sol D). Total FAs are defined as the fatty acid content after acid and/or base hydrolysis of the sample that converts the lipids into their individual fatty acid constituents. Free FAs are defined as those present inherently in the serum as unconjugated, endogenous free acids. Although, not the focus of the study, participants could also report free glycerol and total glycerides in the sera samples (Unknown Serum 2017 A, B, and C) and in the plasma control (SRM 1950). Samples were shipped to participants in March 2017 and results were returned to NIST in May 2017.

The 2017 FAQAP was the last NIST-conducted interlaboratory study devoted entirely to FAs and related analytes in human serum. The FA measurement community is now served through the Health Assessment Measurements Quality Assurance Program (HAMQAP). HAMQAP, in part a collaboration with the National Institutes of Health (NIH) Office of Dietary Supplements (ODS), represents NIST's ongoing and future support of the communities previously served by the Dietary Supplements QAP (DSQAP), Micronutrients Measurement QAP (MMQAP), Vitamin D Metabolites QAP (VitDQAP), as well as the FAQAP. NIST has decades of experience in the administration of these programs. HAMQAP builds on this experience by providing a wide range of matrices and analytes: nutritional and toxic elements, fat- and water-soluble vitamins, FAs, active and/or marker compounds, and contaminants. The HAMQAP design emphasizes emerging and challenging measurements in the dietary supplement, food, and clinical matrix categories. 


\section{Source of Materials}

Study samples were provided to the participants free of charge. Participants received one ampoule of a control plasma material, SRM 1950, three ampoules of an unknown serum labeled as Unknown Serum 2017 Sample A, B, and C, three ampoules of an unknown free fatty acid solution labeled as Unknown Free FA Solution A, B, and C, and one ampoule of an unknown fatty acid/fatty acid methyl ester solution labeled as Unknown FA/FAME Solution D. Additional information of each of the materials is described below.

SRM 1950 Metabolites in Frozen Human Plasma ${ }^{8}$.

Standard Reference Material (SRM) 1950 is a "normal” human plasma intended for validating methods for determining metabolites. The material has certified values for eight total FAs (Table 1) and reference values for an additional 19 total FAs (Table 2). A certified value is a value for which NIST has the highest confidence in its accuracy. A NIST reference value is a noncertified value that is the best estimate of the true value based on available data. The certified values were provided to the participants in the reporting sheet.

Table 1: Certified Values for Fatty Acids in SRM 1950

\begin{tabular}{|l|l|c|c|}
\hline \multicolumn{1}{|c|}{$\begin{array}{c}\text { Lipid } \\
\text { Name }\end{array}$} & \multicolumn{1}{c|}{ Chemical Name / Common Name } & $\begin{array}{c}\text { Mass Fraction } \\
\mu \mathrm{g} / \mathrm{g}\end{array}$ & $\begin{array}{c}\text { Concentration } \\
\mu \mathrm{mol} / \mathrm{L}\end{array}$ \\
\hline C12:0 & Dodecanoic Acid / Lauric Acid & $1.86 \pm 0.11$ & $9.47 \pm 0.57$ \\
\hline C16:0 & Hexadecanoic Acid / Palmitic Acid & $594 \pm 19$ & $2364 \pm 77$ \\
\hline C16:1n7 & $\begin{array}{l}\text { (Z)-9-Hexadecenoic Acid / Palmitoleic } \\
\text { Acid }\end{array}$ & $53.5 \pm 6.4$ & $215 \pm 26$ \\
\hline C18:0 & Octadecanoic Acid / Stearic Acid & $179 \pm 12$ & $644 \pm 41$ \\
\hline C18:3n3 & $\begin{array}{l}\text { Z,Z,Z)-9,12,15-Octadecatrienoic Acid / a- } \\
\text { Linolenic Acid }\end{array}$ & $14.9 \pm 1.0$ & $54.6 \pm 3.6$ \\
\hline C18:1n9 & (Z)-9-Octadecenoic Acid / Oleic Acid & $447 \pm 43$ & $1610 \pm 150$ \\
\hline C18:2n6 & $\begin{array}{l}\text { Z,Z)-9,12-Octadecadienoic Acid / } \\
\text { Linoleic Acid }\end{array}$ & $780 \pm 39$ & $2840 \pm 140$ \\
\hline C22:0 & Docosanoic Acid / Behenic Acid & $15.9 \pm 1.5$ & $47.8 \pm 4.6$ \\
\hline
\end{tabular}


Table 2: Reference Values for Fatty Acids in SRM 1950

\begin{tabular}{|l|l|c|c|}
\hline \multicolumn{1}{|c|}{$\begin{array}{c}\text { Lipid } \\
\text { Name }\end{array}$} & \multicolumn{1}{|c|}{ Chemical Name / Common Name } & $\begin{array}{c}\text { Mass Fraction } \\
\mu \mathrm{g} / \mathrm{g}\end{array}$ & $\begin{array}{c}\text { Concentration } \\
\mu \mathrm{mol} / \mathrm{L}\end{array}$ \\
\hline C14:0 & Tetradecanoic Acid / Myristic Acid & $17.9 \pm 3.8$ & $80 \pm 17$ \\
\hline C14:1 & (Z)-9-Tetradecenoic Acid / Myristoleic Acid & $1.57 \pm 0.03$ & $7.1 \pm 0.1$ \\
\hline C15:0 & Pentadecanoic Acid & $1.08 \pm 0.01$ & $4.56 \pm 0.04$ \\
\hline C17:0 & Heptadecanoic Acid / Margaric Acid & $4.7 \pm 0.2$ & $17.6 \pm 0.7$ \\
\hline C18:3n6 & $\begin{array}{l}\text { (Z,Z,Z)-6,9,12-Octadecatrienoic Acid / } \gamma \text {-Linolenic } \\
\text { Acid }\end{array}$ & $10.9 \pm 2.3$ & $39.9 \pm 8.5$ \\
\hline C18:1n7 & (Z)-11-Octadecenoic Acid / cis-Vaccenic Acid & $37.7 \pm 0.9$ & $136 \pm 3$ \\
\hline C20:0 & Eicosanoic Acid / Arachidic Acid & $5.5 \pm 0.2$ & $18.0 \pm 0.5$ \\
\hline C20:1 & (Z)-11-Eicosenoic Acid / Gondolic Acid & $3.5 \pm 0.1$ & $11.5 \pm 0.5$ \\
\hline C20:2 & (Z,Z)-11,14-Eicosadienoic Acid & $5.7 \pm 0.2$ & $18.8 \pm 0.6$ \\
\hline C20:3n6 & $\begin{array}{l}\text { (Z,Z,Z)-8,11,14-Eicosatrienoic Acid / Homo- } \gamma- \\
\text { Linolenic Acid }\end{array}$ & $41.8 \pm 1.1$ & $139 \pm 4$ \\
\hline C20:4n6 & $\begin{array}{l}\text { (Z,Z,Z,Z)-5,8,11,14-Eicosatetraenoic Acid / } \\
\text { Arachidonic Acid }\end{array}$ & $293 \pm 54$ & $980 \pm 180$ \\
\hline C20:5n3 & $\begin{array}{l}\text { (Z,Z,Z,Z,Z)-5,8,11,14,17-Eicosapentaenoic Acid / } \\
\text { EPA }\end{array}$ & $11.4 \pm 0.1$ & $38.6 \pm 0.5$ \\
\hline C22:1 & (Z)-13-Docosenoic Acid / Erucic Acid & $1.1 \pm 0.4$ & $3.4 \pm 1.3$ \\
\hline C22:4n6 & (Z,Z,Z,Z)-7,10,13,16-Docosatetraenoic Acid & $8.3 \pm 0.2$ & $25.5 \pm 0.6$ \\
\hline C22:5n3 & $\begin{array}{l}\text { (Z,Z,Z,Z,Z)-7,10,13,16,19-Docosapentaenoic Acid/ } \\
\text { DPA }\end{array}$ & $12.5 \pm 0.2$ & $38.5 \pm 0.7$ \\
\hline C22:5n6 & (Z,Z,Z,Z,Z)-4,7,10,13,16-Docosapentaenoic Acid & $6.3 \pm 0.1$ & $19.5 \pm 0.4$ \\
\hline C22:6n3 & $\begin{array}{l}\text { (Z,Z,Z,Z,Z,Z)-4,7,10,13,16,19-Docosahexaenoic } \\
\text { Acid / DHA }\end{array}$ & $37.9 \pm 6.8$ & $118 \pm 21$ \\
\hline C24:0 & Tetracosanoic Acid / Lignoceric Acid & $16.8 \pm 0.9$ & $46.6 \pm 2.6$ \\
\hline C24:1 & (Z)-15-Tetracosenoic Acid / Nervonic Acid & $25.6 \pm 1.2$ & $71.3 \pm 3.2$ \\
\hline & & & \\
\hline
\end{tabular}


Unknown Serum 2017.

This material was prepared from plasma obtained and processed into serum by a contractor in 2007. The serum was bottled in $1 \mathrm{~mL}$ aliquots and stored at $-80{ }^{\circ} \mathrm{C}$ until shipment under dry ice. The approximate fatty acid concentrations $(\mu \mathrm{mol} / \mathrm{L})$ of the unknown serum material were not provided to the participants.

Unknown Free Fatty Acid Solution.

The unknown Free Fatty Acid Solution was prepared by diluting known amounts of nine free FAs in toluene. The solution was prepared at fatty acid levels that are anticipated in serum and plasma samples, and the gravimetric molar concentrations of nine FAs in the Unknown Free Fatty Acid Solution are listed in Table 3. The expanded uncertainty was approximated as $5 \%$ of the mass fraction. Purity of the neat FAs used to prepare the Unknown Free Fatty Acid Solution was not determined. Since only free FAs were used in the solution preparation, the values for total and free FAs are the same (i.e., a method to determine total FAs should get the same value as a method to determine free FAs in this solution). The molar concentrations of the Unknown Free FA Solutions were unknown to the participants.

Table 3: Gravimetric Concentration of Free Fatty Acids in the Unknown FFA Solution.

\begin{tabular}{|c|c|c|}
\hline $\begin{array}{l}\text { Lipid } \\
\text { Name }\end{array}$ & Chemical Name / Common Name & $\begin{array}{c}\text { Concentration } \\
\mu \mathrm{mol} / \mathrm{L}\end{array}$ \\
\hline C18:0 & Octadecanoic Acid / Stearic Acid & $549 \pm 28$ \\
\hline C18:1n7 & (Z)-11-Octadecenoic Acid / cis-Vaccenic Acid & $115 \pm 5.7$ \\
\hline C18:1n9 & (Z)-9-Octadecenoic Acid / Oleic Acid & $1313 \pm 66$ \\
\hline $\mathrm{C} 18: 2 \mathrm{n} 6$ & (Z,Z)-9,12-Octadecadienoic Acid / Linoleic Acid & $2480 \pm 124$ \\
\hline C18:3n3 & $\begin{array}{l}\text { (Z,Z,Z)-9,12,15-Octadecatrienoic Acid / } \alpha \text {-Linolenic } \\
\text { Acid }\end{array}$ & $44.4 \pm 2.2$ \\
\hline $\mathrm{C} 20: 4 \mathrm{n} 6$ & $\begin{array}{l}\text { (Z,Z,Z,Z)-5,8,11,14-Eicosatetraenoic Acid / } \\
\text { Arachidonic Acid }\end{array}$ & $804 \pm 40$ \\
\hline C20:5n3 & $\begin{array}{l}\text { (Z,Z,Z,Z,Z)-5,8,11,14,17-Eicosapentaenoic Acid / } \\
\text { EPA }\end{array}$ & $36.7 \pm 1.8$ \\
\hline C22:5n3 & $\begin{array}{l}\text { (Z,Z,Z,Z,Z)-7,10,13,16,19-Docosapentaenoic Acid / } \\
\text { DPA }\end{array}$ & $38.1 \pm 1.9$ \\
\hline C22:6n3 & $\begin{array}{l}\text { (Z,Z,Z,Z,Z,Z)-4,7,10,13,16,19-Docosahexaenoic Acid } \\
\text { / DHA }\end{array}$ & $98.4 \pm 4.9$ \\
\hline
\end{tabular}


Unknown Fatty Acid/Fatty Acid Methyl Ester Solution.

The Unknown Fatty Acid/Fatty Acid Methyl Ester Solution was prepared by a collaborating laboratory and contains three FAs and 29 fatty acid methyl esters. The gravimetric molar concentration of each component in the solution is unknown to NIST and to the participants. Table 4 lists the FAs and the fatty acid methyl esters that are in the Unknown FA/FAME Solution. Since the solution contained both FAs and fatty acid methyl esters, participants were given the choice of reporting the molar concentration $(\mu \mathrm{mol} / \mathrm{L})$ either as the FA or as the FAME. Some participants reported molar concentrations for both.

Table 4: Fatty Acids and Fatty Acid Methyl Esters in the Unknown FA/FAME Solution.

\begin{tabular}{|c|c|}
\hline Lipid Name & Analyte \\
\hline C14:0 & Myristic acid methyl ester \\
\hline C14:1n5 & Myristoleic acid methyl ester \\
\hline C16:0 & Palmitic acid methyl ester \\
\hline C16:1n7 & Palmitoleic acid methyl ester \\
\hline $\mathrm{C} 16: 1 \mathrm{n} 7 \mathrm{t}$ & Palmitelaidic acid methyl ester \\
\hline C16:1n10 & Sapienic acid \\
\hline C16:1n5 & cis-11-hexadecenoic acid \\
\hline $\mathrm{C} 16: 1 \mathrm{n} 5 \mathrm{t}$ & trans-11-hexadecenoic acid \\
\hline C18:0 & Stearic acid methyl ester \\
\hline C18:1n7 & cis-Vaccenic acid methyl ester \\
\hline C18:1n9 & Oleic acid methyl ester \\
\hline C18:1n9t & Elaidic acid methyl ester \\
\hline C18:1n7t & trans-vaccenic acid methyl ester \\
\hline C18:1n12 & Petroselinic acid methyl ester \\
\hline C18:2n6 & Linoleic acid methyl ester \\
\hline C18:2n6t,9t & Linoelaidic acid methyl ester \\
\hline C18:3n3 & $\alpha$-Linolenic acid methyl ester \\
\hline C18:3n6 & $\gamma$-Linolenic acid methyl ester \\
\hline C20:0 & Arachidic acid methyl ester \\
\hline C20:1n9 & 11-Eicosenoic acid methyl ester \\
\hline C20:2n6 & 11,14-Eicosadienoic acid methyl ester \\
\hline C20:3n6 & homo- $\gamma$-Linolenic acid methyl ester \\
\hline $\mathrm{C} 20: 4 \mathrm{n} 6$ & Arachidonic acid methyl ester \\
\hline $\mathrm{C} 20: 5 n 3$ & Eicosapentaenoic acid methyl ester \\
\hline C22:0 & Docosanoic acid methyl ester \\
\hline C22:1n9 & Docosenoic acid methyl ester \\
\hline $\mathrm{C} 22: 4 \mathrm{n} 6$ & Docosatetraenoic acid methyl ester \\
\hline C22:5n3 & Docosapentaenoic acid methyl ester \\
\hline C22:5n6 & Docosapentaenoic acid methyl ester \\
\hline $\mathrm{C} 22: 6 \mathrm{n} 3$ & Docosahexaenoic acid methyl ester \\
\hline $\mathrm{C} 24: 0$ & Lignoceric acid methyl ester \\
\hline C24:1n9 & Nervonic acid methyl ester \\
\hline
\end{tabular}




\section{Instructions to Participants}

Participants were instructed to analyze a single aliquot from each of the unknown serum samples (Unknown Serum 2017 A, B, and C), one aliquot of SRM 1950, a single aliquot from each of the unknown free fatty acid solutions (Unknown Free FA Sol. A, B, and C), and one aliquot of the unknown fatty acid methyl ester/free fatty acid solution (Unknown FAME/FFA sol. D) using their analytical protocols. Participants could report concentrations for total FAs, free FAs, free glycerol, and total triglycerides. Participants were not required to report values for all measurands listed on the reporting sheet and could add additional compounds when reporting data. Participants were asked to report values in $\mu \mathrm{mol} / \mathrm{L}$ for $\mathrm{FAs}$ and $\mathrm{mmol} / \mathrm{L}$ for glycerol and total triglycerides. The density of each material was provided for those converting from mass fraction to molar concentration. For the Unknown FA/FAME Sol., participants could either choose to report the molar concentration as FA, or FAME (when converting from mass fraction to molar concentration, results can be slightly different depending if the molecular weight of the FA or FAME is used). Some participants reported results for both, while others reported results for one or the other. No calculations were done to convert one from the other after results were reported by the participants. The data file template given to participants for reporting data included multiple sheets for the participants to list information on analytical methods as well as results.

\section{Methods}

Participants were asked to use analytical protocols currently used in their laboratory. For total fatty acid analysis, there were several participants that performed base hydrolysis with $\mathrm{BF}_{3} / \mathrm{methanol}$ derivatization, as well as acid/base hydrolysis and pentafluorobenzylbromide derivatization. Table 5 summarizes the method details for total FAs.

Four participants reported results for free FAs using different measurement protocols. Table 6 summarizes the method details for free FAs. The method summary for the analysis of the fatty acid solutions (Unknown FA Sol. and unknown FA/FAME Sol.) is shown in Table 7. Two participants reported total glycerides and the summary of the methods used is in Table 8. Table 9 shows the summary of the laboratory that reported free glycerol. Full detail method descriptions as reported by each laboratory can be found in Appendix D. 
Table 5: Summary of Participant's Methods for Total Fatty Acids in Serum.

\begin{tabular}{|l|l|l|}
\hline Code & \multicolumn{1}{|c|}{ Sample Preparation Method } & \multicolumn{1}{c|}{ Analytical Method } \\
\hline LC01 & BF3/methanol derivatization & $\begin{array}{l}\text { GC-FID on non-bonded } \\
\text { poly(biscyanopropyl siloxane) }\end{array}$ \\
\hline LC02 & $\begin{array}{l}\text { Acid/base hydrolysis and } \\
\text { pentafluorbenzylbromide derivatization }\end{array}$ & $\begin{array}{l}\text { GC-MS on 5 \% phenyl-95 \% } \\
\text { methylpolysiloxane }\end{array}$ \\
\hline LC04 & BF3/methanol derivatization & GC-FID on FFAP phase column \\
\hline LC05 & BF3/methanol derivatization & GC-FID on FFAP phase column \\
\hline LC07 & $\begin{array}{l}\text { Extraction and acetylchloride } \\
\text { derivatization }\end{array}$ & $\begin{array}{l}\text { GC-FID on (88 \%-cyanopropyl)aryl- } \\
\text { polysiloxane }\end{array}$ \\
\hline LC08 & Acid hydrolysis and methanol & $\begin{array}{l}\text { GC-MS on 5 \% phenyl-95 \% } \\
\text { methylpolysiloxane }\end{array}$ \\
\hline LC09 & $\begin{array}{l}\text { Dry methanolic sodium methoxide plus } \\
\text { methanolic HCL }\end{array}$ & $\begin{array}{l}\text { GC-MS on (50 \% cyanopropylphenyl)- } \\
\text { dimethylpolysiloxane }\end{array}$ \\
\hline LC10 & BF3/methanol derivatization & $\begin{array}{l}\text { GC-FID on (50 \%-cyanopropyl)- } \\
\text { methylpolysiloxane }\end{array}$ \\
\hline LC11 & Liu et al. 2010 & $\begin{array}{l}\text { GC-MS on 5 \% phenyl-95 \% } \\
\text { methylpolysiloxane }\end{array}$ \\
\hline LC12 & $\begin{array}{l}\text { Acid/base hydrolysis and } \\
\text { pentafluorbenzylbromide derivatization }\end{array}$ & GC-MS on a select FAME column \\
\hline LC14 & $\begin{array}{l}\text { Acid/base hydrolysis and } \\
\text { pentafluorbenzylbromide derivatization }\end{array}$ & GC-MS on TG-Polar phase \\
\hline
\end{tabular}

Table 6: Summary of Participant's Methods for Free Fatty Acids in Serum.

\begin{tabular}{|l|l|l|}
\hline Code & \multicolumn{1}{|c|}{ Sample Preparation Method } & \multicolumn{1}{c|}{ Analytical Method } \\
\hline LC01 & BF3 in Methanol/hexane cold temperature & $\begin{array}{l}\text { GC-FID on non-bonded } \\
\text { poly(biscyanopropyl siloxane) }\end{array}$ \\
\hline LC05 & $\begin{array}{l}\text { BF3/methanol derivatization after FFA } \\
\text { separation by thin layer chromatography }\end{array}$ & GC-FID on FFAP phase column \\
\hline LC06 & $\begin{array}{l}\text { Liquid/liquid extraction and no } \\
\text { derivatization }\end{array}$ & GC-MS on FFAP column \\
\hline LC09 & $\begin{array}{l}\text { Extraction and (trimethylsilyl)diazomethane } \\
\text { derivatization }\end{array}$ & $\begin{array}{l}\text { GC-MS on (50 \% cyanopropylphenyl)- } \\
\text { dimethylpolysiloxane }\end{array}$ \\
\hline
\end{tabular}


Table 7: Summary of Participant’s Methods for Solutions

\begin{tabular}{|l|l|l|}
\hline Code & \multicolumn{1}{|c|}{ Sample Preparation Method } & \multicolumn{1}{|c|}{ Analytical Method } \\
\hline LC01 & BF3/methanol derivatization & $\begin{array}{l}\text { GC-FID on non-bonded } \\
\text { poly(biscyanopropyl siloxane) }\end{array}$ \\
\hline LC02 & $\begin{array}{l}\text { Acid/base hydrolysis and } \\
\text { pentafluorbenzylbromide derivatization }\end{array}$ & $\begin{array}{l}\text { GC-MS on 5\% phenyl-95 \% } \\
\text { methylpolysiloxane }\end{array}$ \\
\hline LC04 & BF3/methanol derivatization & GC-FID on FFAP phase column \\
\hline LC05 & BF3/methanol derivatization & GC-FID on FFAP phase column \\
\hline LC07 & extraction and acetylchloride derivatization & $\begin{array}{l}\text { GC-FID on (88 \%-cyanopropyl)aryl- } \\
\text { polysiloxane }\end{array}$ \\
\hline LC08 & Acid hydrolysis and methanol & $\begin{array}{l}\text { GC-MS on 5 \%henyl-95 \% } \\
\text { methylpolysiloxane }\end{array}$ \\
\hline LC09 & $\begin{array}{l}\text { Dilute and (trimethylsilyl)diazomethane } \\
\text { derivatization }\end{array}$ & $\begin{array}{l}\text { GC-MS on (50 \% cyanopropylphenyl)- } \\
\text { dimethylpolysiloxane }\end{array}$ \\
\hline LC10 & BF3/methanol derivatization & $\begin{array}{l}\text { GC-FID on 50 \%-cyanopropyl)- } \\
\text { methylpolysiloxane }\end{array}$ \\
\hline LC11 & Liu et al. 2010 & $\begin{array}{l}\text { GC-MS on 5 \% phenyl-95 \% } \\
\text { methylpolysiloxane }\end{array}$ \\
\hline LC12 & $\begin{array}{l}\text { Acid/base hydrolysis and } \\
\text { pentafluorbenzylbromide derivatization }\end{array}$ & GC-MS on a select FAME column \\
\hline LC14 & $\begin{array}{l}\text { Acid/base hydrolysis and } \\
\text { pentafluorbenzylbromide derivatization }\end{array}$ & GC-MS on TG-Polar phase \\
\hline
\end{tabular}

Table 8: Summary of Participant's Methods for Total Glycerides in Serum.

\begin{tabular}{|c|l|l|}
\hline Code & \multicolumn{1}{|c|}{ Sample Preparation Method } & \multicolumn{1}{c|}{ Analytical Method } \\
\hline LC03 & $\begin{array}{l}\text { Base hydrolysis and pyridine and acetic } \\
\text { anhydride derivatization }\end{array}$ & $\begin{array}{l}\text { GC-MS on 50 \% phenly- } \\
\text { methylpolysiloxane }\end{array}$ \\
\hline LC13 & $\begin{array}{l}\text { Base hydrolysis and pyridine and acetic } \\
\text { anhydride derivatization }\end{array}$ & $\begin{array}{l}\text { GC-MS on 50 \% phenly- } \\
\text { methylpolysiloxane }\end{array}$ \\
\hline
\end{tabular}

Table 9: Summary of Participant Method for Free Glycerol in Serum

\begin{tabular}{|c|l|l|}
\hline Code & \multicolumn{1}{|c|}{ Sample Preparation Method } & \multicolumn{1}{c|}{ Analytical Method } \\
\hline LC13 & $\begin{array}{l}\text { Ethanol precipitation and pyridine and } \\
\text { acetic anhydride derivatization }\end{array}$ & $\begin{array}{l}\text { GC-MS on 50\% phenly- } \\
\text { methylpolysiloxane }\end{array}$ \\
\hline
\end{tabular}




\section{Overview of Data Treatment and Representation}

Data tables and graphs are provided throughout this report using anonymized laboratory codes.

\section{$\underline{\text { Statistics }}$}

Data tables and graphs throughout this report contain information about the relative performance of each laboratory. All calculations were performed in PROLab Plus (QuoData GmbH, Dresden, Germany). The consensus mean and standard deviation are calculated according to the robust Q/Hampel method outlined in ISO 13528:2015(E), Annex C.

\section{Data Tables}

The data submitted by the participants, listed by lab code, are detailed in Appendix A for free and total FAs in plasma and serum samples (Unknown Serum 2017 samples A, B, and C, and SRM 1950), Appendix B for FAs and fatty acid methyl esters in the solutions (Unknown Free FA Sol A, B, and C and Unknown FA/FAME Sol. D), and Appendix C for free glycerol and total glycerides in plasma and serum samples. The results are organized by analyte (indicated on the top line of each table) The laboratory average and standard deviation for samples with multiple measurements (unknown serum 2017 samples and unknown Free Fatty Acid Sol.) are listed. Community statistics are summarized at the bottom of each sample column, including the consensus mean and standard deviation calculated by the robust Q/Hampel method and the number of participants determined in the analysis. Blank spaces in the data table next to a laboratory indicate that that laboratory did not return data for that analyte-sample combination. NIST values listed for SRM 1950 are the certified or reference values and their associated uncertainties as listed in the Certificate of Analysis. ${ }^{8}$

\section{Graphical Representation:}

For each analyte-sample combination when two or more participants reported results, a summary page was generated. At the top of each summary page is the measurand and sample name in bold. Below this information is the consensus mean and uncertainty that was determined using the robust $\mathrm{Q} /$ Hampel method. The median is also listed below the consensus mean. If available, the reference value is listed with its associated uncertainty. This reference value is either the certified or reference value of SRM 1950 or the gravimetric value of the Unknown Free Fatty Acid Solution. The robust standard deviation is listed on the right along with the number of participants in the calculation.

Below this information in the summary page is a graphical representation of the data. In this view, individual laboratory data (circles) are plotted with the individual laboratory standard deviations (rectangles). The blue solid line represents the consensus mean, and the green shaded area represents the $95 \%$ confidence interval for the consensus mean, based on the standard error of the consensus mean. The uncertainty in the consensus mean is calculated using the equation below (ISO 13528), based on the robust standard deviation ( $\mathrm{s}^{*}$, listed as s.d. of ILS at the top of each summary page) and the number of participants reporting data (n).

$$
u_{\text {mean }}=1.25 \frac{s *}{\sqrt{n}}
$$


The black dotted line is the reference value (when available). The blue shaded region represents the expanded uncertainty of the reference value. The solid red lines represent the range of tolerance (values that result in an acceptable $Z$ score, $|Z| \leq 2$ ). If the lower limit is below zero, the lower limit has been set to zero. In this view, the relative locations of individual laboratory data and consensus zones with respect to the reference zone can be compared easily. In most cases, the target zone and the consensus zone overlap, which is the expected result. The results are color coded based on the analytical method used, a short description of the analytical methods is indicated in the legend of the plot. This can be used to determine if there are method biases.

\section{Total Fatty Acids in SRM 1950:}

\section{Results and Discussion}

SRM 1950 was included in the study as a control. Eight total FAs are certified in SRM 1950 and an additional 19 total FAs have reference values (noncertified values). The certified values were listed on the reporting sheet, and hence participants knew the certified values before submitting results. The data tables and the graphical representation summarizing the results submitted by the participants for SRM 1950 are in Appendix A. There was good agreement for most total FAs when comparing the consensus range, the $95 \%$ confidence level of the consensus mean (the green shaded area in the graphical representation of the data), with the certified or reference range. This along with the $95 \%$ confidence level (the blue shaded area in the graphical representation of the data), indicates that the consensus values obtained in this study are comparable to the certified or reference values of SRM 1950. The consensus range for 23 out of 25 FAs overlapped with the certified or reference range (see Table 10). This can be easily viewed in the graphical representation when the green and blue shaded areas overlap. Of these $23 \mathrm{FAs}$, the consensus mean for eight FAs were within the certified or reference range (indicated when the blue solid line that represents the mean lies within the blue shaded region in the graphs). However, for some FAs the uncertainty of the consensus mean was large. This could be due to large variability in the data returned and/or the low number of labs returning data since the uncertainty is inversely proportional to the square root of the number of participants.

The consensus range was outside the reference range for both total pentadecanoic acid (C15:0) and total arachidonic acid (C20:4 n-6) in SRM 1950. It is important to note that for both of these FAs, only a reference (noncertified) value is available in SRM 1950, which may not capture all sources of bias in the uncertainty.

The between-laboratory variability, estimated as the percent relative standard deviation (RSD), for the total FAs that have certified or reference values in SRM 1950 is also summarized in Table 10. The RSD was low for most FAs (less than $30 \%$ ), though ten FAs measured yielded RSDs > $30 \%$ and three FAs had RSDs $>60 \%$ for this control material. The largest RSDs were typically for FAs with low molar concentrations. Between-laboratory variability improved with higher molar concentration analytes. 
Table 10: Comparison of Consensus Mean and Range to SRM 1950 Total FA Values ${ }^{\mathrm{a}}$

\begin{tabular}{|l|l|c|c|c|c|}
\hline \multicolumn{1}{|c|}{ Lipid } & \multicolumn{1}{|c|}{ Common Name } & $\begin{array}{c}\text { SRM 1950 } \\
\text { Certified? }\end{array}$ & $\begin{array}{c}\text { Consensus } \\
\text { Mean }\end{array}$ & $\begin{array}{c}\text { Consensus } \\
\text { Range }\end{array}$ & $\begin{array}{c}\text { RSD }^{\mathrm{c}} \\
\%\end{array}$ \\
\hline C12:0 & Lauric acid & Yes & Below & NA $^{\mathrm{b}}$ & 91 \\
\hline C14:0 & Myristic Acid & No & Within & Overlaps & 30 \\
\hline C14:1n5 & Myristoleic Acid & No & Above & Overlaps & 67 \\
\hline C15:0 & Pentadecanoic Acid & No & Above & Above & 60 \\
\hline C16:0 & Palmitic Acid & Yes & Below & Overlaps & 11 \\
\hline C16:1n7 & Palmitoleic Acid & Yes & Above & Overlaps & 11 \\
\hline C17:0 & Margaric Acid & No & Below & Overlaps & 45 \\
\hline C18:0 & Stearic Acid & Yes & Within & Overlaps & 25 \\
\hline C18:1n7 & cis-Vaccenic Acid & No & Above & Overlaps & 22 \\
\hline C18:1n9 & Oleic Acid & Yes & Within & Overlaps & 16 \\
\hline C18:2n6 & Linoleic Acid & Yes & Within & Overlaps & 14 \\
\hline C18:3n3 & $\alpha$-Linolenic Acid & Yes & Below & Overlaps & 26 \\
\hline C18:3n6 & $\gamma$-Linolenic Acid & No & Within & Overlaps & 25 \\
\hline C20:0 & Arachidic Acid & No & Above & Overlaps & 27 \\
\hline C20:1n9 & 11 -Eicosenoic acid & No & Below & Overlaps & 54 \\
\hline C20:2n6 & $11,14-$-icosadienoic acid & No & Below & Overlaps & 40 \\
\hline C20:3n6 & homo- $\gamma$-Linolenic acid & No & Below & Overlaps & 31 \\
\hline C20:4n6 & Arachidonic Acid & No & Below & Below & 32 \\
\hline C20:5n3 & EPA & No & Below & Overlaps & 55 \\
\hline C22:0 & Docosanoic acid & Yes & Within & Overlaps & 24 \\
\hline C22:4n6 & Docosatetraenoic acid & No & Below & Overlaps & 25 \\
\hline C22:5n3 & DPA & No & Within & Overlaps & 8 \\
\hline C22:5n6 & Docosapentaenoic acid & No & Below & Overlaps & 23 \\
\hline C22:6n3 & DHA & No & Within & Overlaps & 24 \\
\hline C24:0 & Lignoceric acid & No & Below & Overlaps & 32 \\
\hline
\end{tabular}

${ }^{a}$ Interlaboratory Relative Standard Deviation: RSD $=100 *$ SD/Mean.

${ }^{\mathrm{b}}$ Not applicable as the RSD for this FA is too large.

${ }^{\text {c }}$ Comparison between expanded uncertainties of the certificate values with standard deviations of the means for the results from the participating laboratories. 
Total Fatty Acids in Unknown Serum:

Two to eleven participants reported results for 38 total FAs in Unknown serum A, B, and C, which were the same material (see Appendix A). The replicates of the unknown Serum 2017 study are plotted for each laboratory in the graphical representation in Appendix A (individual laboratory data points are represented by circles, with the individual laboratory standard deviations represented by the rectangles). While for most participants and FAs, the intralaboratory repeatability is good, the interlaboratory variability for many of the FAs in the Unknown Serum 2017 samples are large (Table 11). There are 12 total FAs with a RSD of less than $30 \%$, and 26 total FAs with a RSD greater than $30 \%$, and 12 of the 26 FAs have RSD greater than $60 \%$. Similar to SRM 1950, many of the FAs in the unknown serum samples that have high variability have low molar concentrations and/or a low number of participants returning data. It is difficult to draw conclusions from data for analytes with low participation rates. Robust statistics does not work well with a low number of participants, and hence, outlier results may influence the consensus mean and standard deviation more compared to when larger number of participants report results. Generally good agreement was observed for the total FAs measured by most participants. Out of the 38 total FAs in Table 11, eight or more participants submitted data for 19 FAs. Out of these 19 FAs, 11 of the FAs had RSD below $30 \%$.

There is both large interlaboratory variability and low participation rates for the trans FAs. For example, four participants reported results for elaidic acid (C18: 1n9t); two participants reported molar concentrations higher than $100 \mu \mathrm{mol} / \mathrm{L}$, one laboratory reported a molar concentration around $40 \mu \mathrm{mol} / \mathrm{L}$, and the fourth reported a molar concentration of $1 \mu \mathrm{mol} / \mathrm{L}$. One possible reason for the high variability is due to inadequate separation of the trans FAs from other components. Other reasons of high variability in the data could be due to method biases, such as incomplete hydrolyses of the triglycerides and phospho-lipids containing the FAs. 
Table 11: Total Fatty Acids in Unknown Serum

\begin{tabular}{|c|c|c|c|c|}
\hline Lipid & Common Name & $N$ & $\begin{array}{c}\text { Mean } \pm \text { SD } \\
\mu \mathrm{mol} / \mathrm{L}\end{array}$ & $\begin{array}{c}\text { RSD } \\
\%\end{array}$ \\
\hline C10:0 & Decanoic acid & 4 & $7.0 \pm 8.1$ & 117 \\
\hline C12:0 & Lauric acid & 6 & $15 \pm 16$ & 103 \\
\hline C12:1n1 & 11-Dodecenoic acid & 2 & $2.7 \pm 1.5$ & 55 \\
\hline C14:0 & Myristic acid & 10 & $121 \pm 43$ & 36 \\
\hline C14:1n5 & Myristoleic acid & 7 & $8.5 \pm 8.7$ & 102 \\
\hline C15:0 & Pentadecanoic acid & 6 & $20 \pm 14$ & 72 \\
\hline C16:0 & Palmitic acid & 10 & $2699 \pm 445$ & 16 \\
\hline C16:1n7 & Palmitoleic acid & 11 & $236 \pm 49$ & 21 \\
\hline $\mathrm{C} 16: 1 \mathrm{n} 7 \mathrm{t}$ & Palmitelaidic acid & 3 & $22 \pm 22$ & 102 \\
\hline C17:0 & Margaric acid & 5 & $27 \pm 16$ & 59 \\
\hline C18:0 & Stearic acid & 11 & $829 \pm 169$ & 20 \\
\hline C18:1n7 & cis-Vaccenic acid & 7 & $171 \pm 57$ & 33 \\
\hline C18:1n9 & Oleic acid & 11 & $2103 \pm 430$ & 20 \\
\hline C18:1n9t & Elaidic acid & 4 & $77 \pm 140$ & 182 \\
\hline C18:2n6 & Linoleic acid & 11 & $3433 \pm 726$ & 21 \\
\hline C18:2n6t,9t & Linoelaidic acid & 5 & $70 \pm 80$ & 113 \\
\hline C18:3n3 & $\alpha$-Linolenic acid & 11 & $56 \pm 20$ & 35 \\
\hline C18:3n6 & $\gamma$-Linolenic acid & 11 & $39 \pm 22$ & 56 \\
\hline C18:4n3 & Stearidonic acid & 3 & $4.3 \pm 4.4$ & 102 \\
\hline C19:0 & Nonadecanoic acid & 2 & $2.8 \pm 8.9$ & 314 \\
\hline C20:0 & Arachidic acid & 9 & $25.4 \pm 8.3$ & 33 \\
\hline C20:1n9 & 11-Eicosenoic acid & 9 & $11.3 \pm 6.0$ & 53 \\
\hline $\mathrm{C} 20: 2 \mathrm{n} 6$ & 11,14-Eicosadienoic acid & 9 & $20.2 \pm 8.6$ & 42 \\
\hline C20:3n3 & 11,14,17-Eicosatrienoic acid & 2 & $4.6 \pm 7.4$ & 161 \\
\hline C20:3n6 & homo- $\gamma$-Linolenic acid & 11 & $152 \pm 30$ & 20 \\
\hline C20:3n9 & 5,8,11-Eicosatrienoic acid & 3 & $11.0 \pm 6.8$ & 62 \\
\hline $\mathrm{C} 20: 4 \mathrm{n} 6$ & Arachidonic acid & 11 & $681 \pm 110$ & 16 \\
\hline C20:5n3 & Eicosapentaenoic acid & 10 & $44 \pm 19$ & 42 \\
\hline C22:0 & Docosanoic acid & 8 & $61 \pm 22$ & 37 \\
\hline C22:1n9 & Docosenoic acid & 6 & $7.5 \pm 8.0$ & 107 \\
\hline $\mathrm{C} 22: 2 \mathrm{n} 6$ & Docosadienoic acid & 2 & $2.7 \pm 1.4$ & 52 \\
\hline C22:4n6 & Docosatetraenoic acid & 9 & $23.2 \pm 4.3$ & 19 \\
\hline $\mathrm{C} 22: 5 \mathrm{n} 3$ & Docosapentaenoic acid & 9 & $41.3 \pm 3.0$ & 7 \\
\hline C22:5n6 & Docosapentaenoic acid & 9 & $26.6 \pm 7.9$ & 30 \\
\hline C22:6n3 & Docosahexaenoic acid & 10 & $168 \pm 20$ & 12 \\
\hline C23:0 & Tricosanoic acid & 4 & $20 \pm 14$ & 70 \\
\hline C24:0 & Lignoceric acid & 8 & $47 \pm 13$ & 27 \\
\hline C24:1n9 & Nervonic acid & 8 & $71 \pm 10$ & 14 \\
\hline
\end{tabular}


Free Fatty Acids in SRM 1950 and Unknown Serum A, B, C:

There was a low number of labs returning data for free FAs in the SRM 1950 and Unknown Serum 2017 samples (see Appendix A for data tables and graphical representation). Table 12 lists the consensus mean and standard deviation of free FAs determined in both SRM 1950 and in the Unknown Serum 2017 samples. Free FAs with the largest standard deviations were typically observed for the FAs present at lower molar concentrations. It is interesting to note that different methods were used by the four participants that reported free FAs. Most of the participants took care to avoid exposing the samples to elevated temperatures during the extraction steps, so as not to induce deconjugation of the FAs from triglycerides or phospholipids.

Table 12: Consensus Mean of Select Free Fatty Acids in SRM 1950 And Unknown Serum

\begin{tabular}{|c|c|c|c|c|c|}
\hline & & \multicolumn{2}{|r|}{ SRM 1950} & \multicolumn{2}{|c|}{ Unknown Serum } \\
\hline Lipid & Common Name & $N$ & $\begin{array}{c}\text { Mean } \pm \text { SD } \\
\mu \mathrm{mol} / \mathrm{L}\end{array}$ & $N$ & $\begin{array}{c}\text { Mean } \pm \text { SD } \\
\mu \mathrm{mol} / \mathrm{L}\end{array}$ \\
\hline C14:0 & Myristic acid & 4 & $8.0 \pm 1.3$ & 4 & $9.3 \pm 1.4$ \\
\hline C16:0 & Palmitic acid & 4 & $156 \pm 91$ & 4 & $178 \pm 16$ \\
\hline C16:1n7 & Palmitoleic acid & 4 & $17.4 \pm 3.6$ & 4 & $14.0 \pm 2.6$ \\
\hline C18:0 & Stearic Acid & 4 & $32 \pm 36$ & 4 & $46 \pm 34$ \\
\hline C18:1n7 & cis-Vaccenic Acid & 2 & $16 \pm 12$ & 3 & $10 \pm 14$ \\
\hline C18:1n9 & Oleic Acid & 4 & $149 \pm 30$ & 4 & $133 \pm 18$ \\
\hline C18:2n6 & Linoleic Acid & 4 & $70 \pm 43$ & 4 & $109 \pm 20$ \\
\hline C18:3n3 & $\alpha$-Linolenic Acid & 2 & $3.9 \pm 2.0$ & 2 & $3.4 \pm 1.8$ \\
\hline C18:3n6 & $\gamma$-Linolenic Acid & 3 & $0.997 \pm 0.025$ & 3 & $0.86 \pm 0.38$ \\
\hline C20:3n6 & homo- $\gamma$-Linolenic acid & 3 & $1.3 \pm 1.0$ & 3 & $2.2 \pm 2.0$ \\
\hline C20:4n6 & Arachidonic acid & 3 & $7.2 \pm 6.9$ & 3 & $12.8 \pm 6.0$ \\
\hline C20:5n3 & EPA (C20:5 n-3) & 2 & $0.48 \pm 0.50$ & 3 & $0.92 \pm 1.0$ \\
\hline C22:4n6 & Docosatetraenoic acid & 2 & $0.83 \pm 0.36$ & 3 & $0.45 \pm 0.61$ \\
\hline C22:5n3 & DPA & 3 & $1.25 \pm 1.4$ & 3 & $0.79 \pm 0.38$ \\
\hline C22:5n6 & Docosapentaenoic acid & 2 & $0.546 \pm 0.080$ & 3 & $0.54 \pm 0.41$ \\
\hline C22:6n3 & DHA & 3 & $2.4 \pm 2.4$ & 3 & $3.8 \pm 1.4$ \\
\hline
\end{tabular}


Fatty Acid Solutions A,B,C:

Eight to twelve participants reported results for the Unknown Fatty Acid Sol. A, B, and C, which were of the same material (See Appendix B). The consensus range (95\% confidence interval of the consensus mean) overlapped with the gravimetric value with an estimated expanded uncertainty of $5 \%$ of the gravimetric value for all FAs. All but two consensus mean values fell within the estimated gravimetric uncertainty except for EPA and DPA. The interlaboratory variability for the unknown fatty acid sol. for all FAs but EPA were less than $30 \%$.

Table 13: Comparison of Consensus Ranges to Gravimetric Target in Unknown FFA Solution

\begin{tabular}{|l|l|c|c|c|}
\hline \multicolumn{1}{|c|}{ Lipid } & \multicolumn{1}{c|}{ Common Name } & $\begin{array}{c}\text { Consensus } \\
\text { Mean }\end{array}$ & $\begin{array}{c}\text { Consensus } \\
\text { Range }\end{array}$ & $\begin{array}{c}\text { RSD } \\
\%\end{array}$ \\
\hline C18:0 & Stearic Acid & within & overlaps & 17 \\
\hline C18:1n7 & cis-Vaccenic Acid & within & overlaps & 19 \\
\hline C18:1n9 & Oleic Acid & within & overlaps & 17 \\
\hline C18:2n6 & Linoleic Acid & within & overlaps & 15 \\
\hline C18:3n3 & $\alpha$-Linolenic Acid & within & overlaps & 22 \\
\hline C20:4n6 & Arachidonic Acid & Within & overlaps & 24 \\
\hline C20:5n3 & EPA & below & overlaps & 34 \\
\hline C22:5n3 & DPA & Slightly below & overlaps & 13 \\
\hline C22:6n3 & DHA & within & overlaps & 17 \\
\hline
\end{tabular}

Unknown Fatty Acid/Fatty Acid Methyl Ester Solution D:

The Unknown Fatty Acid/Fatty Acid Methyl Ester Sol. consisted of three FAs and 29 fatty acid methyl esters. Since the solution contained both FAs and fatty acid methyl esters, participants were given the choice of reporting the molar concentration either as the fatty acid or as the fatty acid methyl ester. The choice was given to the participants in case participants were converting mass fraction values to molar concentration. However, the choice of reporting the molar concentration as the FA or the FAME confused some of the participants. Some participants reported the molar concentration as the FA or the FAME, while some participants reported both (see Appendix B). For the participants that reported results for both, it was unclear how these values were determined. For at least one laboratory, the results before and after methylation were reported as the FAME and FA respectively.

Two to four participants reported results for the Unknown Fatty Acid/FAME Sol. as FAME. Results for nine selected FAs in Table 14 suggest generally good agreement between the labs with all RSD less than $30 \%$ except for arachidonic acid (43\%). Six to four participants reported molar concentration of the Unknown Fatty Acid/FAME Sol. as $\mu \mathrm{mol} / \mathrm{L}$ of the FA. The selected nine analytes had RSD of less than $30 \%$, indicating good between lab agreement. 
Table 14: Consensus Mean of Fatty Acid Methyl Esters in FAME Solution D

\begin{tabular}{|l|l|}
\hline \multicolumn{1}{|c|}{ Lipid } & \multicolumn{1}{c|}{ Common Name } \\
\hline C18:0 & Stearic Acid \\
\hline C18:1n7 & cis-Vaccenic Acid \\
\hline C18:1n9 & Oleic Acid \\
\hline C18:2n6 & Linoleic Acid \\
\hline C18:3n3 & $\alpha$-Linolenic Acid \\
\hline C20:4n6 & Arachidonic Acid \\
\hline C20:5n3 & EPA \\
\hline C22:5n3 & DPA \\
\hline C22:6n3 & DHA \\
\hline
\end{tabular}

\begin{tabular}{|l|c|}
\hline \multicolumn{2}{|c|}{ Reported as FAME } \\
\hline$N$ & $\begin{array}{c}\text { Mean } \pm \text { SD } \\
\text { mol/L }\end{array}$ \\
\hline 4 & $559 \pm 29$ \\
\hline 2 & $220 \pm 37$ \\
\hline 4 & $1814 \pm 360$ \\
\hline 4 & $2239 \pm 457$ \\
\hline 4 & $100 \pm 28$ \\
\hline 4 & $469 \pm 202$ \\
\hline 4 & $223 \pm 38$ \\
\hline 3 & $53.3 \pm 7.4$ \\
\hline 4 & $310 \pm 45$ \\
\hline
\end{tabular}

\begin{tabular}{|c|c|}
\hline \multicolumn{2}{|c|}{ Reported as FA } \\
\hline$N$ & $\begin{array}{c}\text { Mean } \pm \text { SD } \\
\mu \mathrm{mol} / \mathrm{L}\end{array}$ \\
\hline 8 & $534 \pm 80$ \\
\hline 6 & $216 \pm 59$ \\
\hline 8 & $1647 \pm 246$ \\
\hline 8 & $2082 \pm 229$ \\
\hline 7 & $112 \pm 3.2$ \\
\hline 8 & $435 \pm 50$ \\
\hline 7 & $190 \pm 37$ \\
\hline 7 & $52.5 \pm 5.8$ \\
\hline 7 & $279 \pm 44$ \\
\hline
\end{tabular}

Total Glycerides and Free Glycerol in SRM 1950 and Unknown Serum 2017:

Two participants reported values for total glycerides for SRM 1950 and for Unknown Serum 2017 Samples (see Appendix C). The consensus mean $(1.10 \mathrm{mmol} / \mathrm{L} \pm 0.03 \mathrm{mmol} / \mathrm{L})$ for total glyceride in SRM 1950 is comparable to the certified value in SRM $1950(1.12 \mathrm{mmol} / \mathrm{L} \pm 02 \mathrm{mmol} / \mathrm{L})$. The two participants also reported similar values for total glycerides in the Unknown Serum 2017 samples as well $(1.53 \mathrm{mmol} / \mathrm{L}$ and $1.54 \mathrm{mmol} / \mathrm{L})$. Only one of these participants also reported a value for free glycerol.

\section{Acknowledgements}

Financial support for this intercomparison exercise was provided by the National Institutes of Health (NIH) Office of Dietary Supplements (ODS).

We thank our NIST colleague David Duewer for his editorial assistance. The time and effort of the analysts and management of the participating laboratories are gratefully acknowledged. 


\section{References}

1 Chu, X.; He, X.; Shi, Z.; Li, C.; Guo, F.; et al.; Ursolic acid increases energy expenditure through enhancing free fatty acid uptake and $\beta$-oxidation via an UCP3/AMPK-dependent pathway in skeletal muscle. Mol Nutr Food Res 59(8); pp. 1491-1503 (2015). https://doi.org/10.1002/mnfr.201400670

2 Nishikiori, M.; Iizuka, H.; Ichiba, H.; Sadamoto, K.; Fukushima, T.; Determination of free fatty acids in human serum by HPLC with fluorescence detection. J Chromatogr Sci 53(4); pp. 537-541 (2015). https://doi,org/10.1093/chromsci/bmu081

3 Guo, S.-X.; Yan, Y.-Z.; Mu, L.-T.; Niu, Q.; He, J.; et al.; Association of Serum Free Fatty Acids with Hypertension and Insulin Resistance among Rural Uyghur Adults in Far Western China. Int J Environ Res Public Health 12(6); pp. 6582-6590 (2015). https://doi.org/10.3390/ijerph120606582

4 Salerno, A.; Fragasso, G.; Esposito, A.; Canu, T.; Lattuada, G.; et al. Effects of short-term manipulation of serum FFA concentrations on left ventricular energy metabolism and function in patients with heart failure: no association with circulating bio-markers of inflammation. Acta Diabetol 52(4):753-761 (2015) https://doi.org/10.1007/s00592-014-0695-7

5 Schantz, M.M.; Powers, C.D.; Schleicher, R.L. Interlaboratory Analytical Comparison Study of Total Fatty Acid Concentrations in Human Serum: Results for Exercise 01: QA12FASER01. NISTIR 7953, Gaithersburg, MD (2013) https://doi.org/10.6028/NIST.IR.7953

6 Schantz, M.M. Interlaboratory Analytical Comparison Study of Fatty Acid Concentrations in Human Serum-Results for Exercise 02: QA15FASER02. NISTIR 8086, Gaithersburg, MD (2015) https://doi.org/10.6028/NIST.IR.8086

7 Schantz, M.M. Interlaboratory Analytical Comparison Study of Fatty Acid Concentrations in Human Serum-Results for Exercise 03: QA16FASER03. NISTIR 8146, Gaithersburg, MD (2016) https://doi.org/10.6028/NIST.IR.8146

8 Certificate of Analysis, SRM 1950 Metabolites in Human Plasma. Gaithersburg, MD USA (2016) https://www-s.nist.gov/srmors/view_cert.cfm?srm=1950 


\section{Appendix A: Free and Total Fatty Acids in Plasma and Serum}

\section{List of Tables}

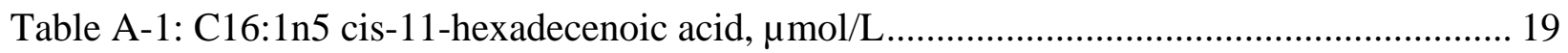

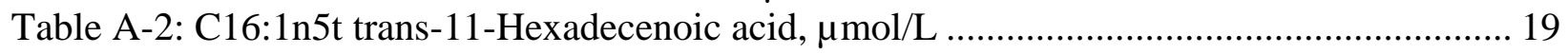

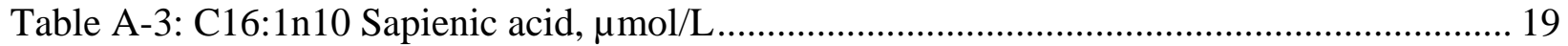

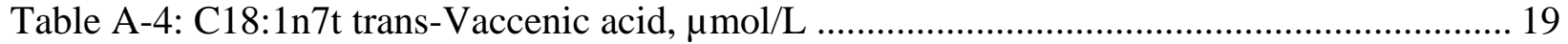

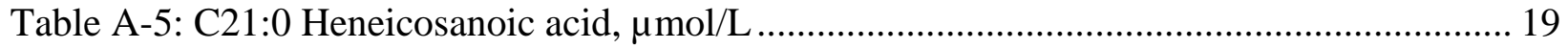

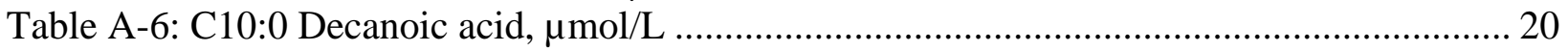

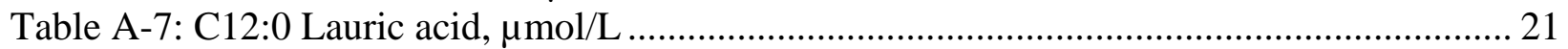

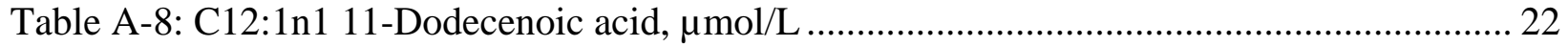

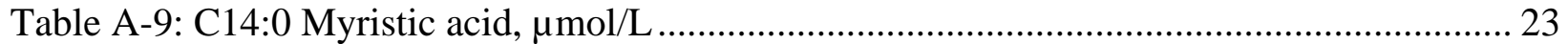

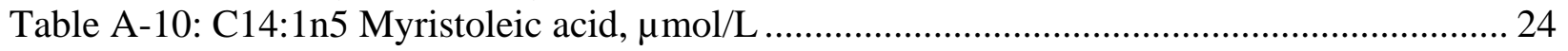

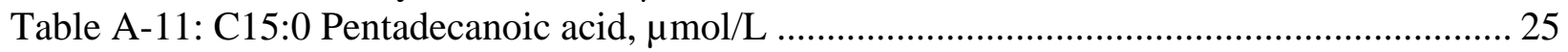

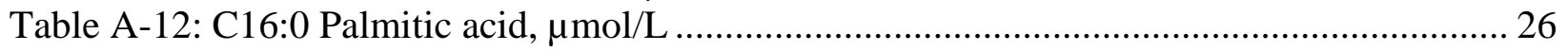

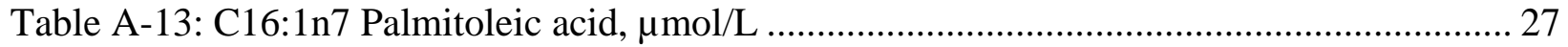

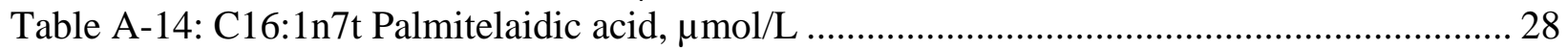

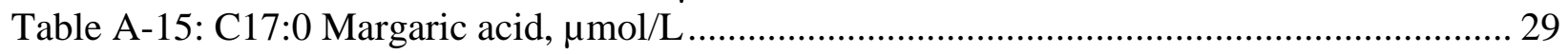

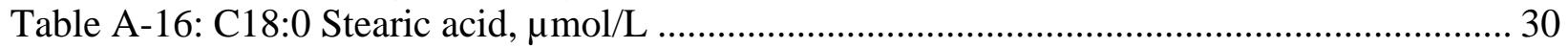

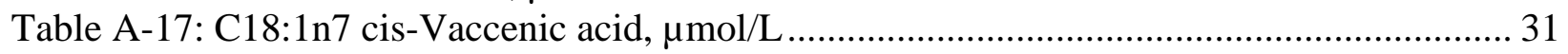

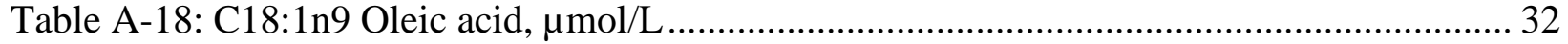

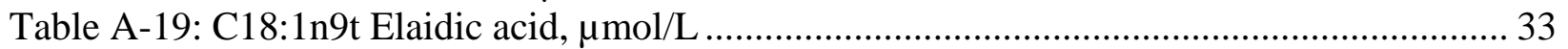

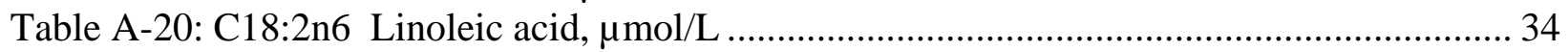

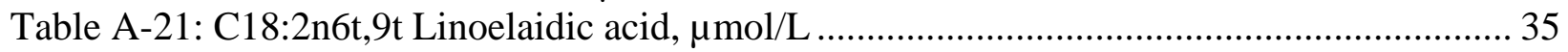

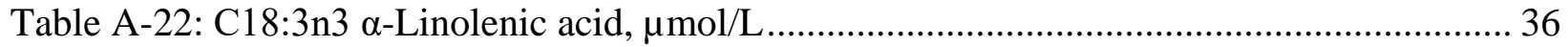

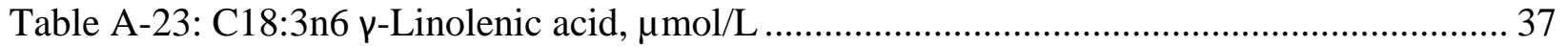

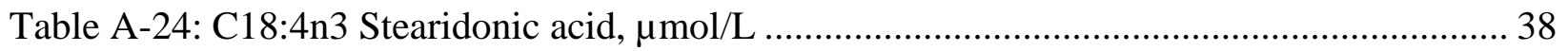

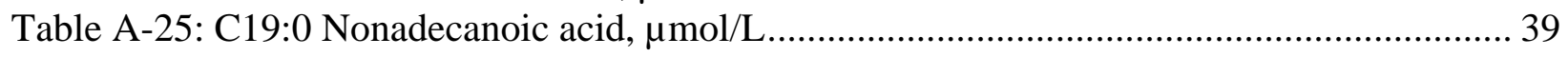

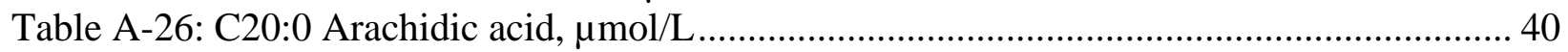

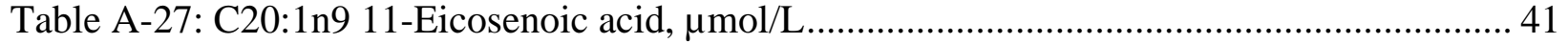

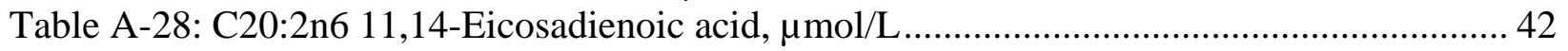

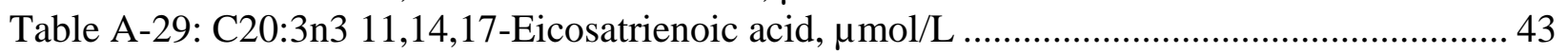

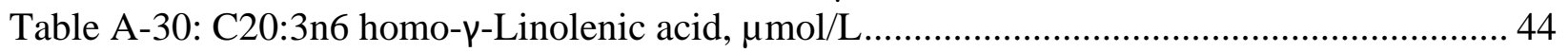

Table A-31: C20:3n9 5,8,11-Eicosatrienoic acid (Mead acid), pmol/L ......................................... 45

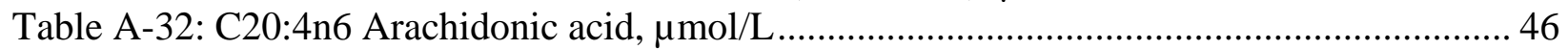

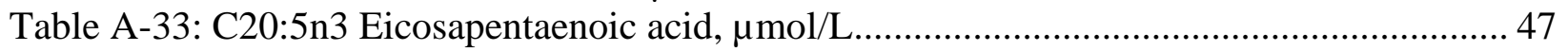

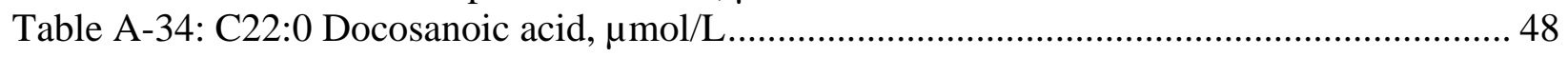

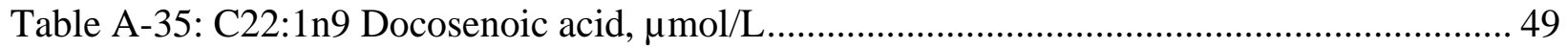

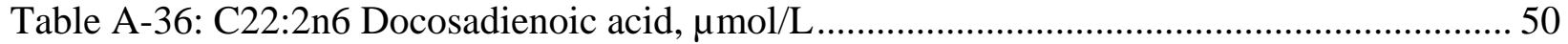

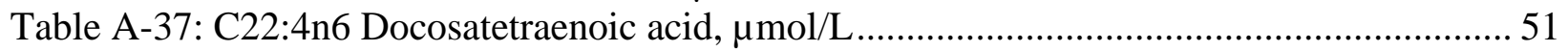

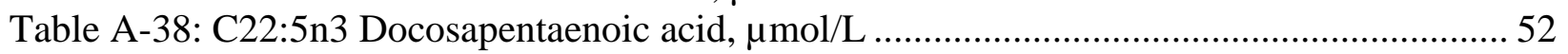

Table A-39: C22:5n6 Docosapentaenoic acid, $\mu \mathrm{mol} / \mathrm{L}$................................................................... 53

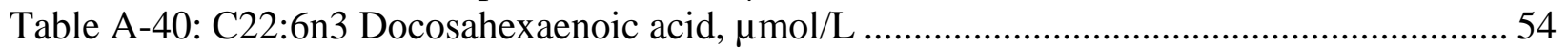

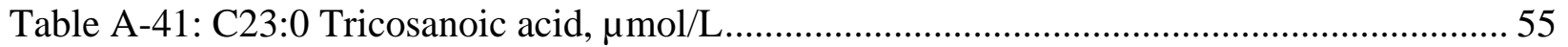

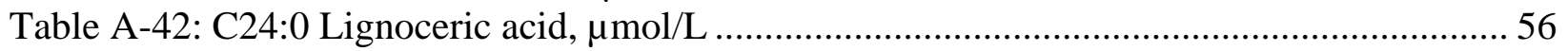

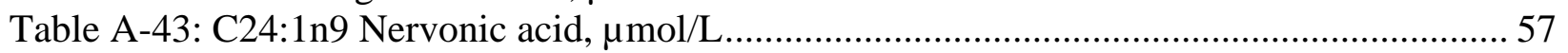




\section{Acronyms Used in Tables}

$\begin{array}{ll}\text { Avg } & \text { Mean } \\ \text { FA } & \text { Total fatty acid } \\ \text { FFA } & \text { Free fatty acid } \\ \text { Lab } & \text { Participant code } \\ <\text { LOD } & \text { Less than the limit of detection } \\ \text { N } & \text { Number of quantitative results } \\ \text { SD } & \text { Standard deviation }\end{array}$

Table A-1: C16:1n5 cis-11-hexadecenoic acid, $\mu \mathrm{mol} / \mathrm{L}$

\begin{tabular}{|c|c|c|c|c|c|c|c|c|c|c|c|c|}
\hline & FA & \multicolumn{5}{|c|}{2017 Unknown, FA } & FFA & \multicolumn{5}{|c|}{2017 Unknown, FFA } \\
\hline Lab & SRM 1950 & A & B & C & Avg & SD & SRM 1950 & A & B & C & Avg & SD \\
\hline LC05 & 6.80 & 9.04 & 8.32 & 8.63 & 8.67 & 0.36 & 0.578 & & & & & \\
\hline
\end{tabular}

Table A-2: C16:1n5t trans-11-Hexadecenoic acid, $\mu \mathrm{mol} / \mathrm{L}$

\begin{tabular}{|c|c|c|c|c|c|c|c|c|c|c|c|c|}
\hline & FA & \multicolumn{5}{|c|}{2017 Unknown, FA } & FFA & \multicolumn{5}{|c|}{2017 Unknown, FFA } \\
\hline Lab & SRM 1950 & A & $\mathrm{B}$ & $\mathrm{C}$ & Avg & SD & SRM 1950 & A & $\mathrm{B}$ & $\mathrm{C}$ & Avg & SD \\
\hline LC05 & 6.92 & 12.4 & 11.6 & 11.9 & 12.0 & 0.40 & 0.969 & 1.06 & 1.39 & 1.24 & 1.23 & 0.17 \\
\hline
\end{tabular}

Table A-3: C16:1n10 Sapienic acid, $\mu \mathrm{mol} / \mathrm{L}$

\begin{tabular}{|c|c|c|c|c|c|c|c|c|c|c|c|c|}
\hline & FA & \multicolumn{5}{|c|}{2017 Unknown, FA } & FFA & \multicolumn{5}{|c|}{2017 Unknown, FFA } \\
\hline Lab & SRM 1950 & A & $\mathrm{B}$ & $\mathrm{C}$ & Avg & SD & SRM 1950 & A & B & $\mathrm{C}$ & Avg & SD \\
\hline LC05 & 49.0 & 53.6 & 54.6 & 54.7 & 54.3 & 0.57 & & & & & & \\
\hline
\end{tabular}

Table A-4: C18:1n7t trans-Vaccenic acid, $\mu \mathrm{mol} / \mathrm{L}$

\begin{tabular}{|c|c|c|c|c|c|c|c|c|c|c|c|c|}
\hline & FA & \multicolumn{5}{|c|}{2017 Unknown, FA } & FFA & \multicolumn{5}{|c|}{2017 Unknown, FFA } \\
\hline $\mathrm{Lab}$ & SRM 1950 & A & $\mathrm{B}$ & $\mathrm{C}$ & Avg & SD & SRM 1950 & A & $\mathrm{B}$ & $\mathrm{C}$ & Avg & SD \\
\hline LC12 & 20.4 & 35.4 & 36.0 & 35.5 & 35.6 & 0.33 & & & & & & \\
\hline
\end{tabular}

Table A-5: C21:0 Heneicosanoic acid, $\mu \mathrm{mol} / \mathrm{L}$

\begin{tabular}{|c|c|c|c|c|c|c|c|c|c|c|c|c|}
\hline & FA & \multicolumn{5}{|c|}{2017 Unknown, FA } & FFA & \multicolumn{5}{|c|}{2017 Unknown, FFA } \\
\hline Lab & SRM 1950 & A & $\mathrm{B}$ & $\mathrm{C}$ & Avg & SD & SRM 1950 & A & $\mathrm{B}$ & $\mathrm{C}$ & Avg & SD \\
\hline LC05 & 2.09 & 3.02 & 3.55 & 2.81 & 3.13 & 0.38 & 0.201 & 0.286 & 0.225 & 0.180 & 0.230 & 0.053 \\
\hline
\end{tabular}


Table A-6: C10:0 Decanoic acid, $\mu \mathrm{mol} / \mathrm{L}$

\begin{tabular}{|c|c|c|c|c|c|c|c|c|c|c|c|c|}
\hline & FA & \multicolumn{5}{|c|}{2017 Unknown, FA } & FFA & \multicolumn{5}{|c|}{2017 Unknown, FFA } \\
\hline Lab & SRM 1950 & A & B & $\mathrm{C}$ & Avg & SD & SRM 1950 & A & B & C & Avg & SD \\
\hline LC02 & & 8.00 & 8.00 & 8.00 & 8.00 & 0.00 & & & & & & \\
\hline LC05 & 11.5 & 11.1 & 11.7 & 14.4 & 12.37 & 1.8 & & & & & & \\
\hline LC08 & 1.85 & 3.21 & 3.49 & 3.19 & 3.30 & 0.17 & & & & & & \\
\hline LC10 & $<\mathrm{LOD}$ & $<\mathrm{LOD}$ & $<\mathrm{LOD}$ & $<\mathrm{LOD}$ & $<\mathrm{LOD}$ & & & & & & & \\
\hline LC14 & 2.61 & 4.14 & 4.25 & 4.00 & 4.13 & 0.13 & & & & & & \\
\hline & Avg 5.32 & & & & Avg & 6.95 & & & & & & \\
\hline & SD & & & & SD & 8.1 & & & & & & \\
\hline & $\mathrm{N}$ & & & & $\mathrm{N}$ & 4 & & & & & & \\
\hline
\end{tabular}

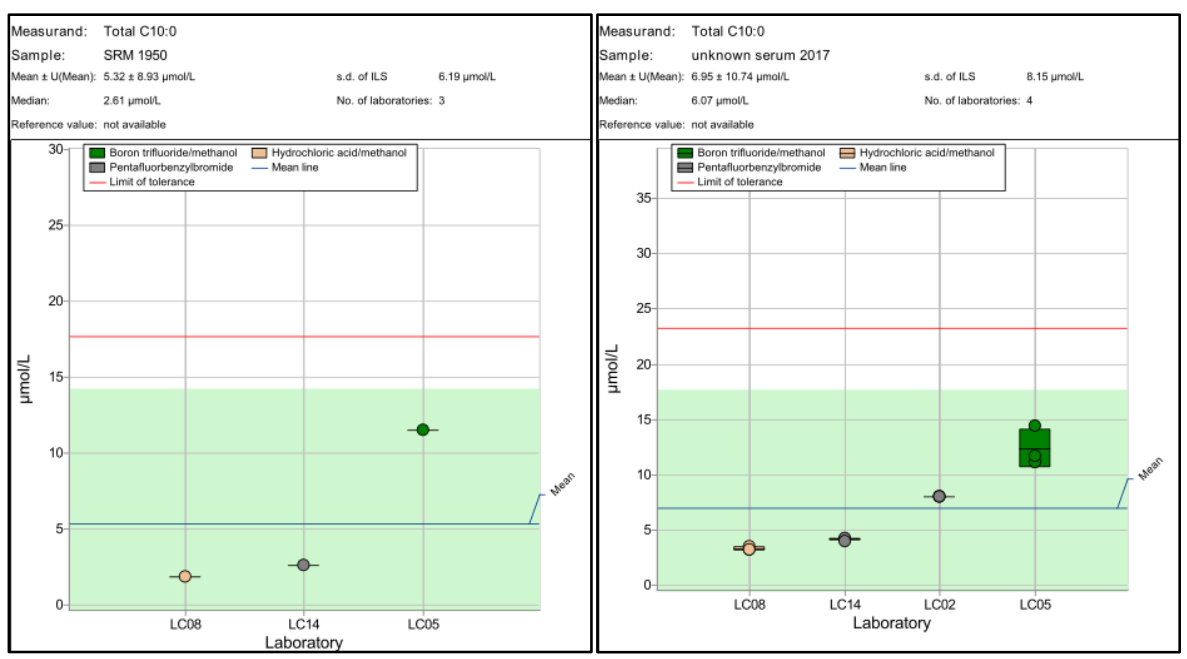


Table A-7: C12:0 Lauric acid, $\mu \mathrm{mol} / \mathrm{L}$

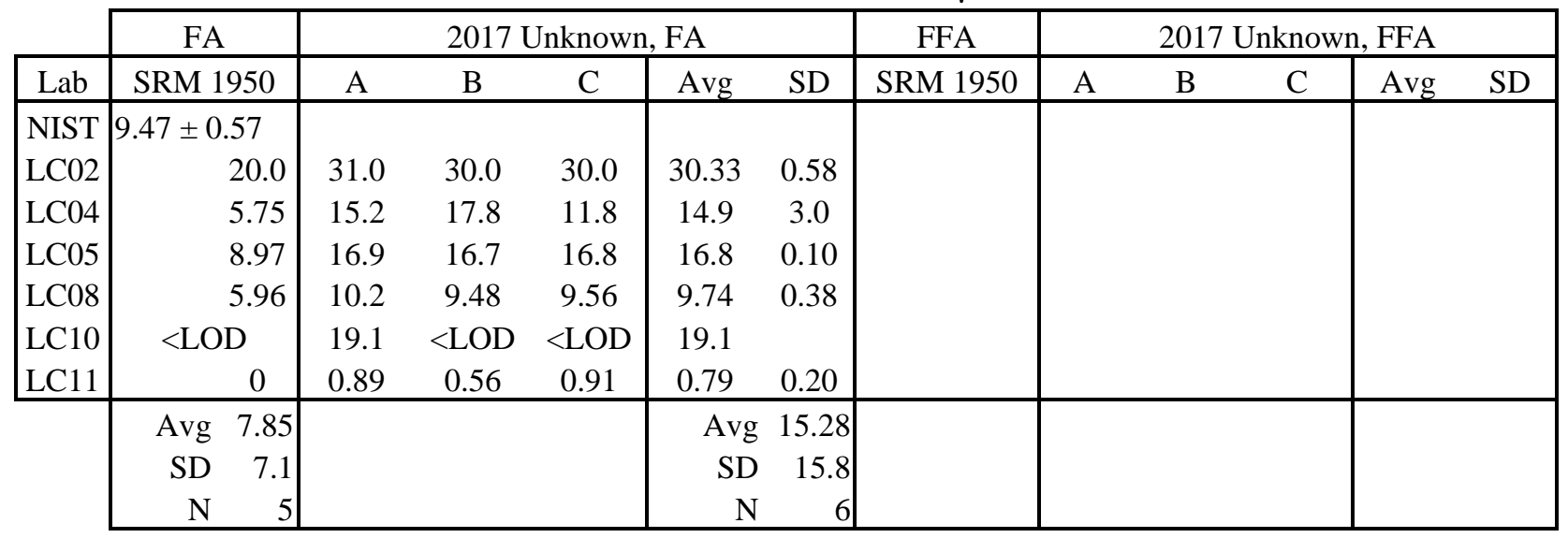
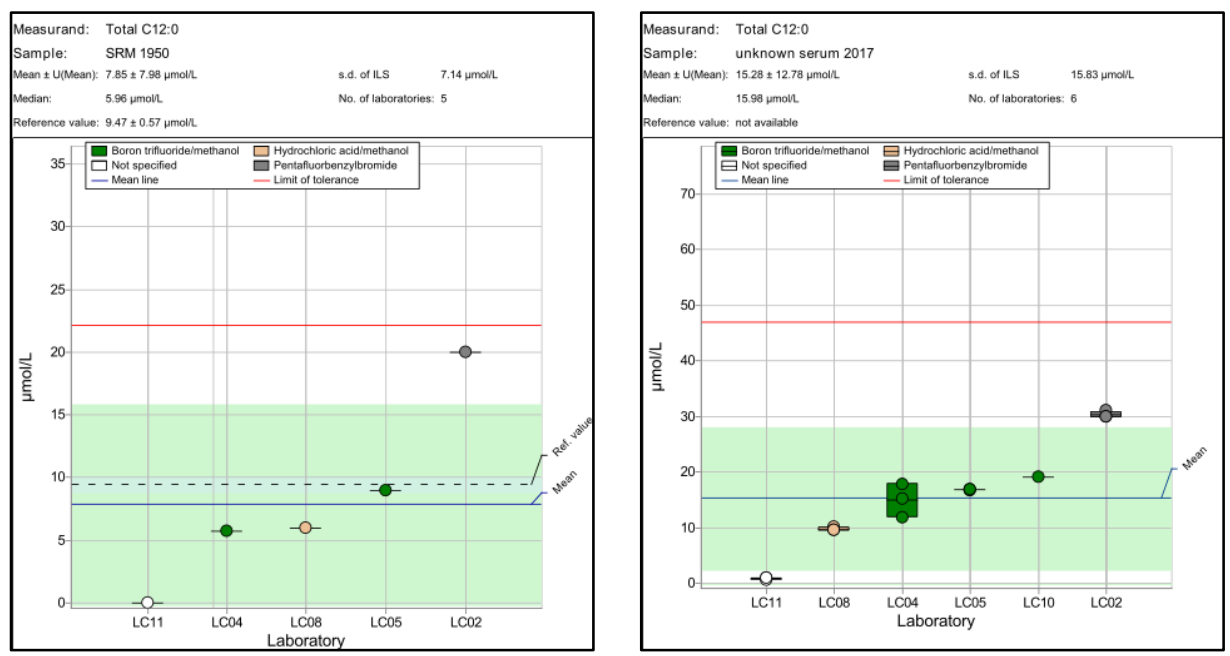
Table A-8: C12:1n1 11-Dodecenoic acid, $\mu \mathrm{mol} / \mathrm{L}$

\begin{tabular}{|c|c|c|c|c|c|c|c|c|c|c|c|c|}
\hline & FA & \multicolumn{5}{|c|}{2017 Unknown, FA } & FFA & \multicolumn{5}{|c|}{2017 Unknown, FFA } \\
\hline Lab & SRM 1950 & A & $\mathrm{B}$ & $\mathrm{C}$ & Avg & SD & SRM 1950 & A & B & $\mathrm{C}$ & Avg & SD \\
\hline LC02 & & 2.9 & 2.8 & 2.8 & 2.83 & 0.06 & & & & & & \\
\hline LC05 & 1.756 & 1.69 & 2.22 & 3.72 & 2.54 & 1.05 & & & & & & \\
\hline & $\mathrm{N}$ & & & & $\begin{array}{r}\text { Avg } \\
\text { SD } \\
\mathrm{N}\end{array}$ & $\begin{array}{r}2.65 \\
1.5 \\
2\end{array}$ & & & & & & \\
\hline
\end{tabular}

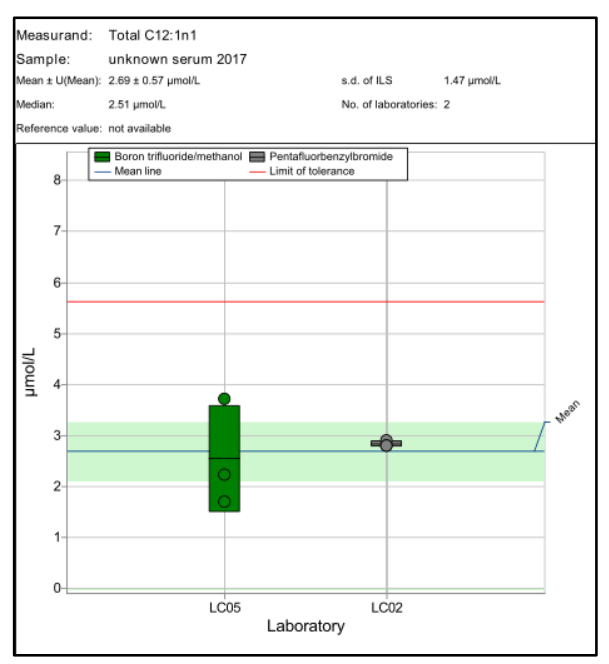


Table A-9: C14:0 Myristic acid, $\mu \mathrm{mol} / \mathrm{L}$

\begin{tabular}{|c|c|c|c|c|c|c|c|c|c|c|c|c|c|}
\hline \multirow[b]{2}{*}{ Lab } & FA & \multicolumn{5}{|c|}{2017 Unknown, FA } & \multicolumn{2}{|c|}{ FFA } & \multicolumn{5}{|c|}{2017 Unknown, FFA } \\
\hline & SRM 1950 & A & $\mathrm{B}$ & $\mathrm{C}$ & Avg & SD & & М 1950 & A & $\mathrm{B}$ & $\mathrm{C}$ & Avg & SD \\
\hline NIST & $80 \pm 17$ & & & & & & & & & & & & \\
\hline LC01 & 109 & 125 & 144 & 135 & 135 & 9.3 & & 8.07 & 8.33 & 8.07 & 7.98 & 8.13 & 0.18 \\
\hline LC02 & & 181 & 176 & 177 & 178 & 2.6 & & & & & & & \\
\hline LC04 & 58.2 & 84.1 & 95.2 & 91.3 & 90.2 & 5.7 & & & & & & & \\
\hline LC05 & 104 & 146 & 144 & 144 & 145 & 1.1 & & 8.87 & 10.1 & 10.2 & 10.4 & 10.2 & 0.16 \\
\hline LC06 & & & & & & & & 7.27 & 9.37 & 8.39 & 7.80 & 8.52 & 0.79 \\
\hline LC07 & 111 & 167 & 156 & 162 & 162 & 5.5 & & & & & & & \\
\hline LC08 & 121 & 72.0 & 63.9 & 60.8 & 65.5 & 5.8 & & & & & & & \\
\hline LC09 & 62.4 & 89.4 & 89.1 & 93.0 & 90.5 & 2.1 & & 7.84 & 9.91 & 9.58 & 10.9 & 10.1 & 0.69 \\
\hline LC10 & 92.2 & 141 & 130 & 135 & 135 & 5.5 & & & & & & & \\
\hline LC11 & 45.7 & 57.8 & 75.9 & 70.3 & 68.0 & 9.3 & & & & & & & \\
\hline LC12 & 96.8 & 141 & 144 & 142 & 142 & 1.4 & & & & & & & \\
\hline & Avg 89.3 & & & & Avg & 121 & Avg & 8.01 & & & & Avg & 9.25 \\
\hline & SD 26 & & & & SD & 43 & SD & 1.3 & & & & SD & 1.3 \\
\hline & $\mathrm{N}$ & & & & $\mathrm{N}$ & 10 & $\mathrm{~N}$ & 4 & & & & $\mathrm{~N}$ & 4 \\
\hline
\end{tabular}
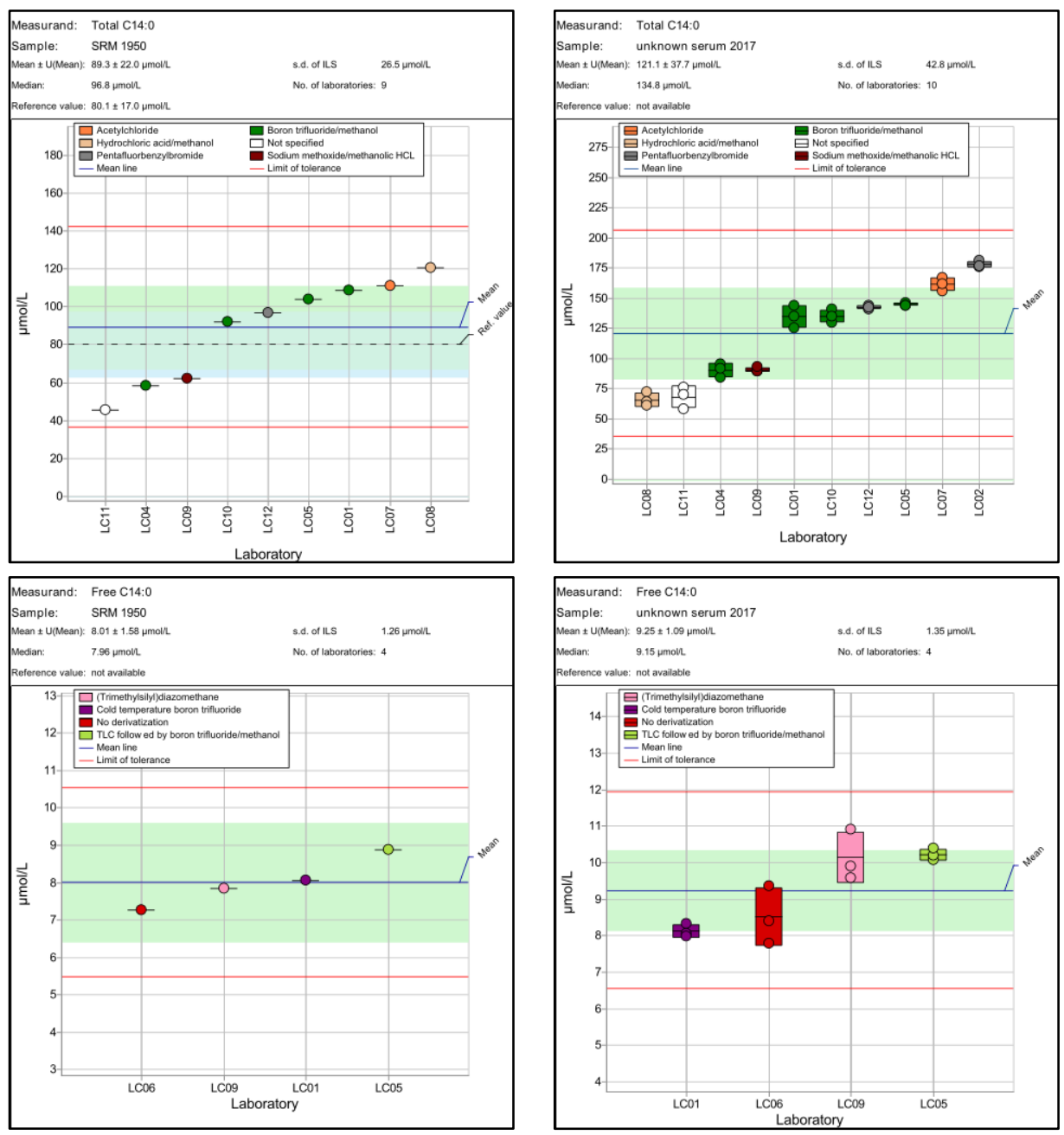
Table A-10: C14:1n5 Myristoleic acid, $\mu \mathrm{mol} / \mathrm{L}$

\begin{tabular}{|c|c|c|c|c|c|c|c|c|c|c|c|c|}
\hline & FA & & 2017 & knov & & & FFA & & 2017 & nknov & FFA & \\
\hline Lab & \multirow{2}{*}{$\frac{\text { SRM } 1950}{7.1 \pm 0.1}$} & A & B & $\mathrm{C}$ & Avg & SD & SRM 1950 & A & B & $\mathrm{C}$ & Avg & SD \\
\hline NIST & & & & & & & \multirow{8}{*}{1.28} & \multirow{8}{*}{1.44} & \multirow{8}{*}{1.29} & \multirow{8}{*}{1.17} & \multirow{8}{*}{1.30} & \multirow{8}{*}{0.13} \\
\hline LC02 & & 21.0 & 21.0 & 21.0 & 21.0 & 0.00 & & & & & & \\
\hline LC05 & 7.85 & 10.4 & 9.94 & 10.1 & 10.1 & 0.21 & & & & & & \\
\hline LC08 & 16.3 & 3.78 & 2.90 & 1.20 & 2.63 & 1.3 & & & & & & \\
\hline LC09 & 5.46 & 5.18 & 3.18 & 3.73 & 4.03 & 1.0 & & & & & & \\
\hline LC11 & 1.75 & 0.960 & 0.810 & 1.51 & 1.09 & 0.37 & & & & & & \\
\hline LC12 & 9.57 & 12.3 & 12.5 & 12.6 & 12.5 & 0.11 & & & & & & \\
\hline LC14 & 7.15 & 8.18 & 8.12 & 7.39 & 7.90 & 0.44 & & & & & & \\
\hline & Avg 7.95 & & & & Avg & 8.47 & & & & & & \\
\hline & SD & & & & SD & 8.7 & & & & & & \\
\hline & $\mathrm{N}$ & & & & $\mathrm{N}$ & 7 & $\mathrm{~N}$ & & & & $\Gamma$ & 1 \\
\hline
\end{tabular}
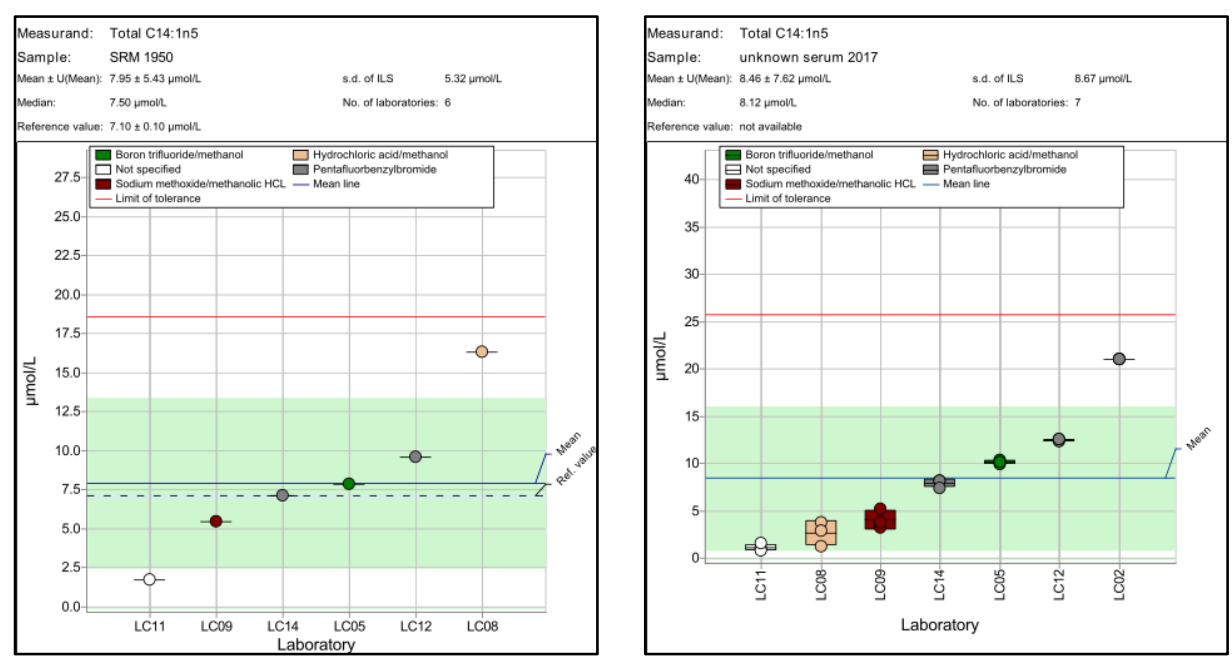
Table A-11: C15:0 Pentadecanoic acid, $\mu \mathrm{mol} / \mathrm{L}$

\begin{tabular}{|c|c|c|c|c|c|c|c|c|c|c|c|c|}
\hline & FA & & 2017 & know & FA & & FFA & & 2017 & nknov & FFA & \\
\hline Lab & SRM 1950 & A & B & $\mathrm{C}$ & Avg & SD & SRM 1950 & A & $\mathrm{B}$ & $\mathrm{C}$ & Avg & SD \\
\hline NIST & $4.56 \pm 0.04$ & & & & & & \multirow{7}{*}{0.360} & \multirow{7}{*}{0.775} & \multirow{7}{*}{0.879} & \multirow{7}{*}{2.47} & \multirow{7}{*}{1.37} & \multirow{7}{*}{0.95} \\
\hline LC05 & 21.1 & 29.4 & 29.8 & 29.5 & 29.6 & 0.19 & & & & & & \\
\hline LC08 & 6.47 & 11.5 & 11.7 & 10.6 & 11.3 & 0.56 & & & & & & \\
\hline LC09 & 4.60 & 9.67 & 8.55 & 9.60 & 9.28 & 0.63 & & & & & & \\
\hline LC10 & 20.8 & 26.7 & 29.2 & 30.4 & 28.8 & 1.9 & & & & & & \\
\hline LC11 & 7.26 & 12.6 & 21.0 & 20.4 & 18.0 & 4.7 & & & & & & \\
\hline \multirow[t]{4}{*}{ LC14 } & 15.2 & 21.5 & 20.9 & 19.8 & 20.7 & 0.85 & & & & & & \\
\hline & Avg 12.6 & & & & Avg & 19.6 & & & & & & \\
\hline & SD 7.5 & & & & SD & 14 & & & & & & \\
\hline & $\mathrm{N}$ & & & & $\mathrm{N}$ & & $\mathrm{N}$ & & & & I & 1 \\
\hline
\end{tabular}
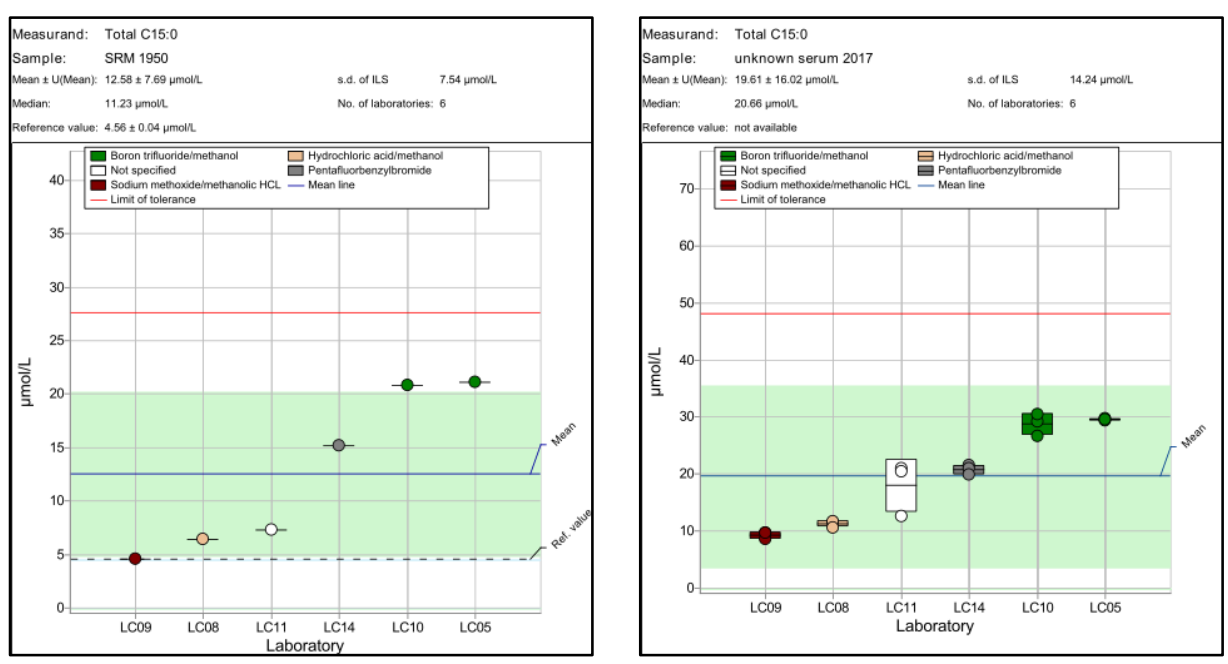
Table A-12: C16:0 Palmitic acid, $\mu \mathrm{mol} / \mathrm{L}$

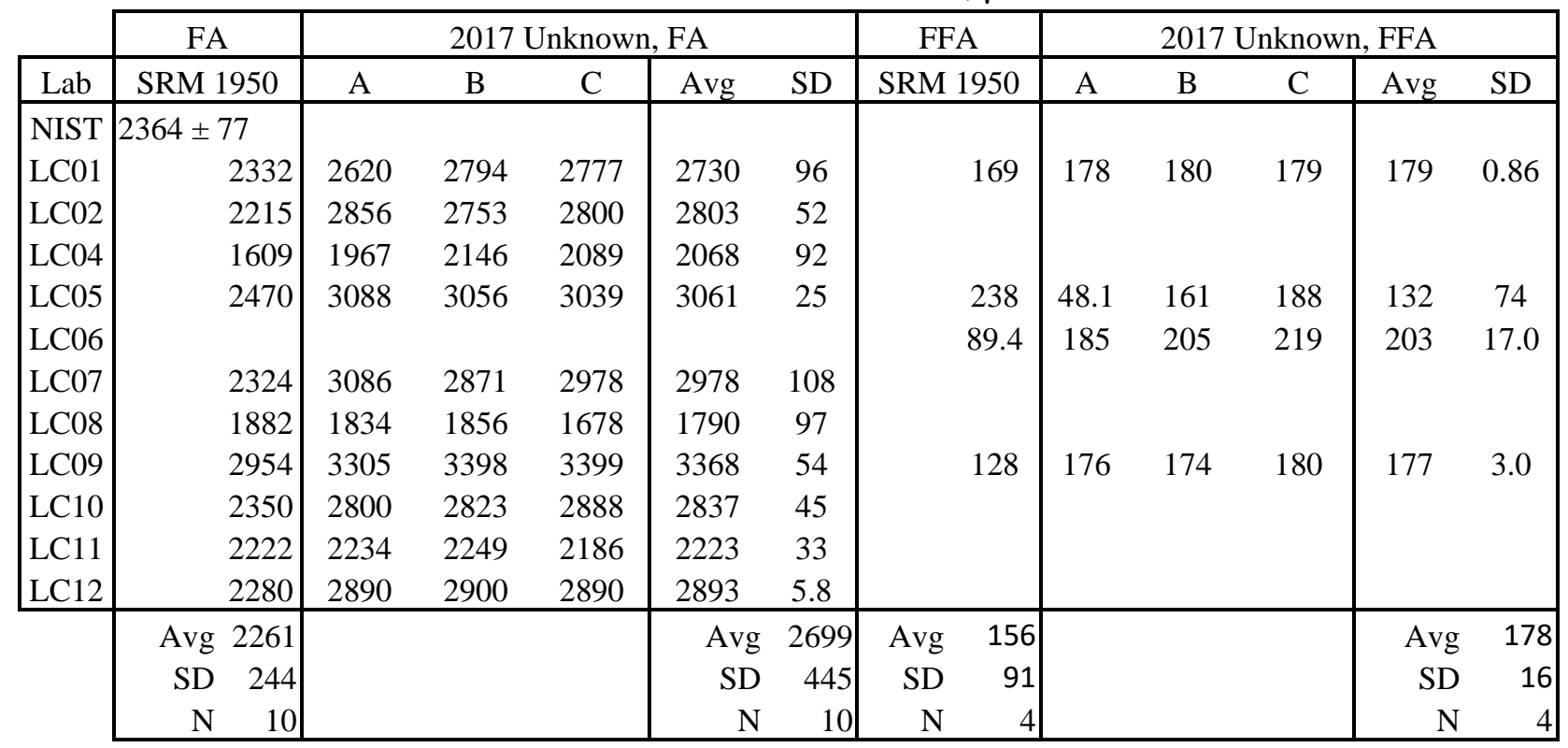
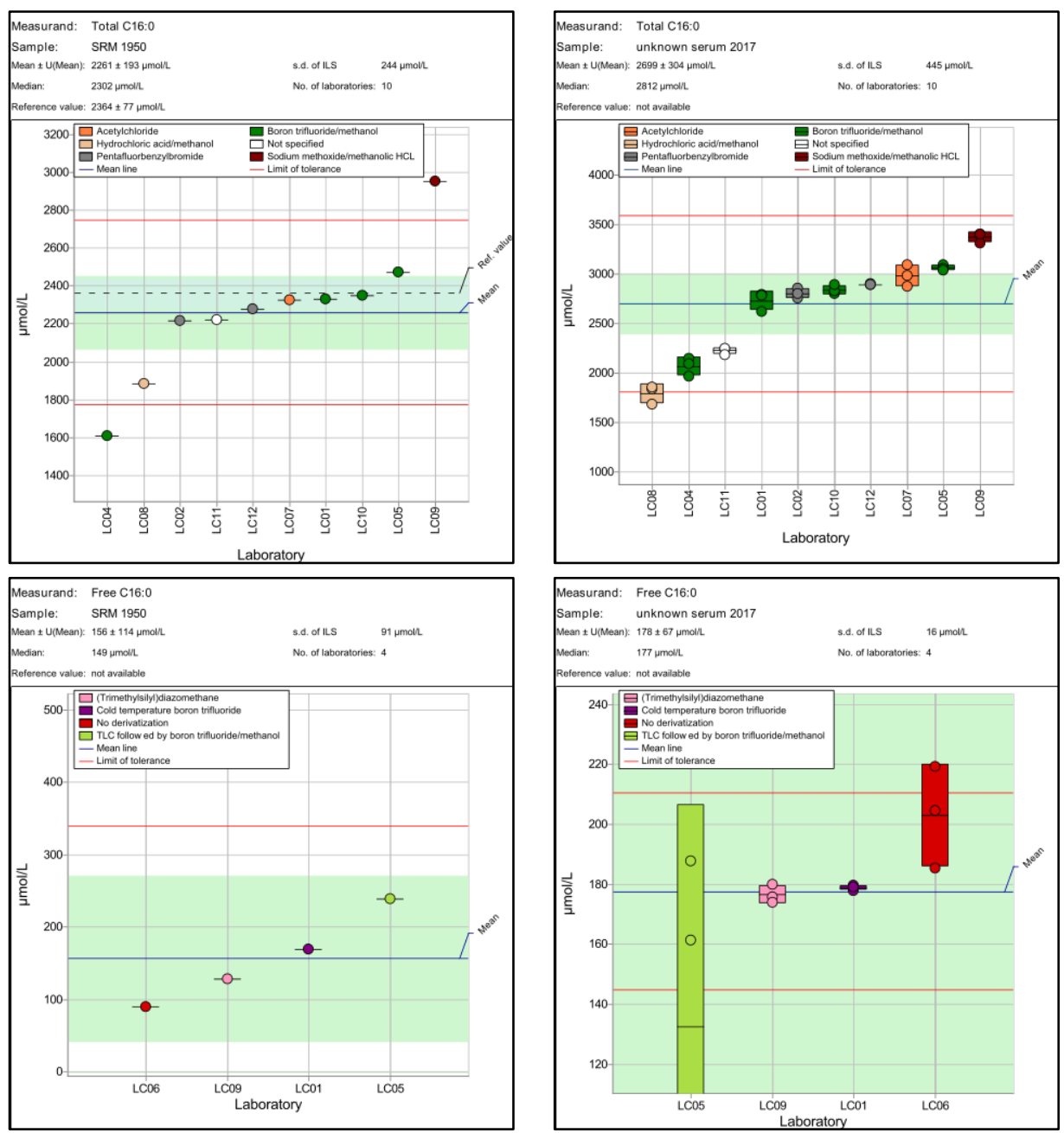
Table A-13: C16:1n7 Palmitoleic acid, $\mu \mathrm{mol} / \mathrm{L}$

\begin{tabular}{|c|c|c|c|c|c|c|c|c|c|c|c|c|c|}
\hline & \multirow{2}{*}{$\frac{\text { FA }}{\text { SRM } 1950}$} & \multicolumn{5}{|c|}{2017 Unknown, FA } & \multicolumn{2}{|l|}{ FFA } & \multicolumn{5}{|c|}{2017 Unknown, FFA } \\
\hline Lab & & A & B & $\mathrm{C}$ & Avg & SD & SRM 19 & 950 & A & B & $\mathrm{C}$ & Avg & SD \\
\hline NIST & $215 \pm 26$ & & & & & & & & & & & & \\
\hline LC01 & 251 & 237 & 238 & 241 & 238 & 2.0 & & 8.7 & 12.5 & 13.8 & 13.9 & 13.4 & 0.76 \\
\hline LC02 & 251 & 303 & 293 & 292 & 296 & 6.1 & & & & & & & \\
\hline LC04 & 155 & 158 & 177 & 168 & 167 & 9.6 & & & & & & & \\
\hline LC05 & 276 & 285 & 282 & 279 & 282 & 3.0 & & 16.7 & 15.6 & 15.8 & 16.1 & 15.9 & 0.26 \\
\hline LC06 & & & & & & & & 19.0 & 9.5 & 13.4 & 17.7 & 13.6 & 4.09 \\
\hline LC07 & 258 & 280 & 257 & 271 & 269 & 12 & & & & & & & \\
\hline LC08 & 231 & 123 & 95 & 94 & 104 & 16 & & & & & & & \\
\hline LC09 & 280 & 247 & 249 & 261 & 253 & 7.6 & & 15.1 & 14.1 & 13.1 & 12.7 & 13.3 & 0.71 \\
\hline LC10 & 264 & 258 & 258 & 260 & 259 & 1.4 & & & & & & & \\
\hline LC11 & 207 & 209 & 246 & 242 & 232 & 20 & & & & & & & \\
\hline LC12 & 249 & 245 & 245 & 246 & 245 & 0.70 & & & & & & & \\
\hline LC14 & 240 & 200 & 193 & 182 & 192 & 9.0 & & & & & & & \\
\hline & Avg 247 & & & & Avg & & Avg & 17.4 & & & & Avg & 14.03 \\
\hline & SD $\quad 28$ & & & & SD & 49 & SD & 3.6 & & & & SD & 2.61 \\
\hline & 11 & & & & $\mathrm{~N}$ & 11 & $\mathrm{~N}$ & 4 & & & & $\mathrm{~N}$ & 4 \\
\hline
\end{tabular}
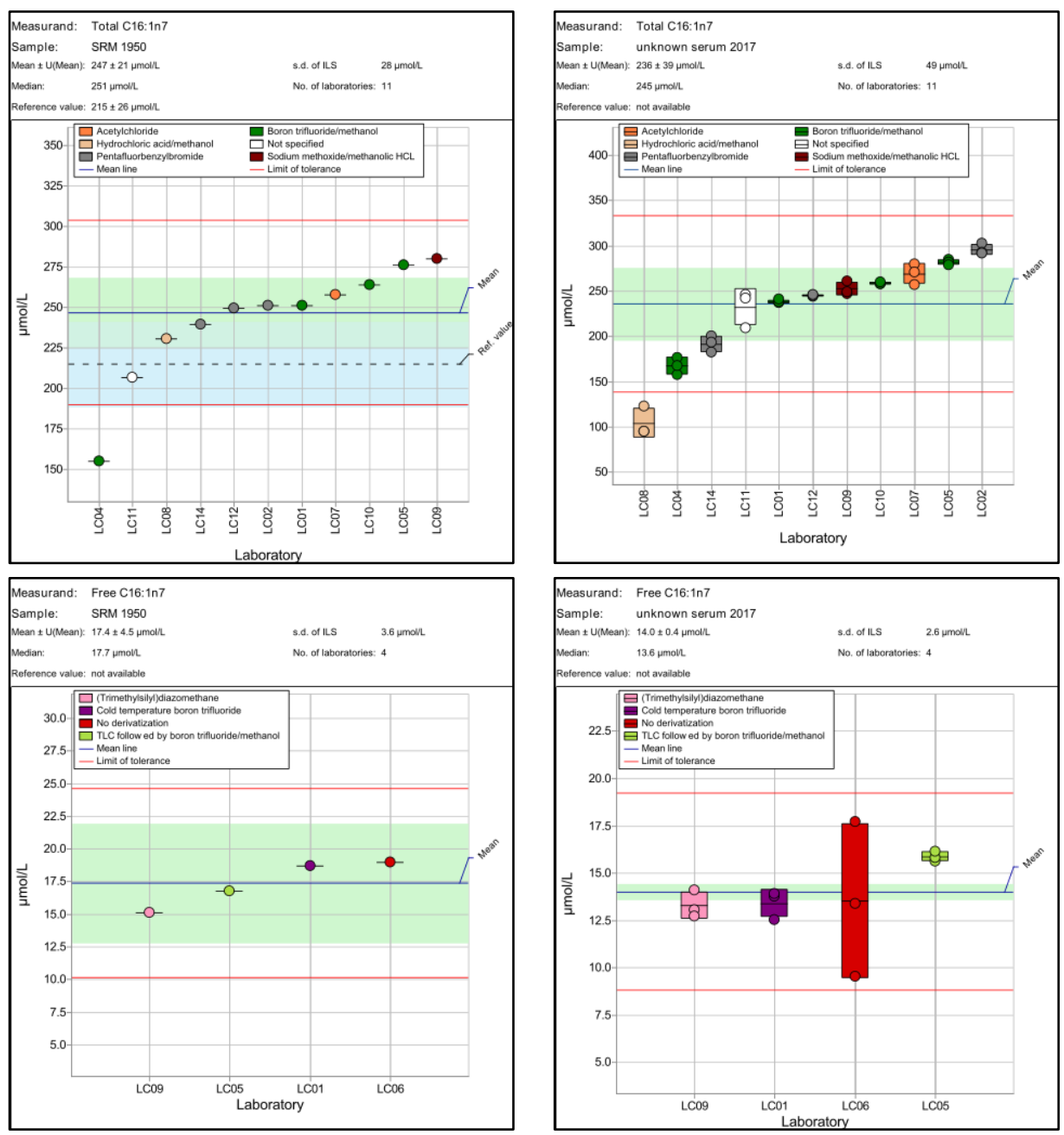
Table A-14: C16:1n7t Palmitelaidic acid, $\mu \mathrm{mol} / \mathrm{L}$

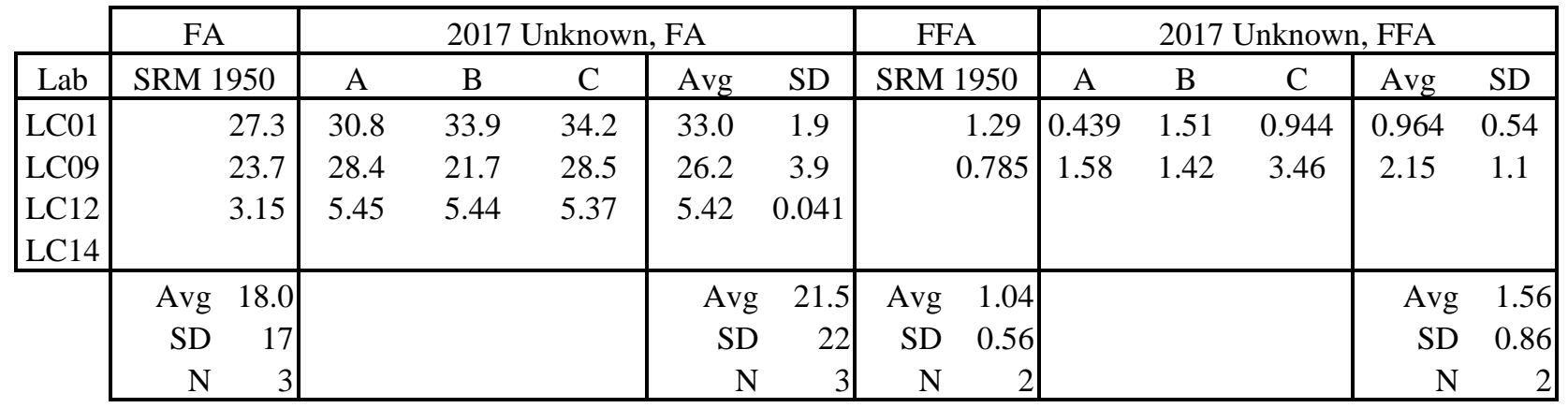
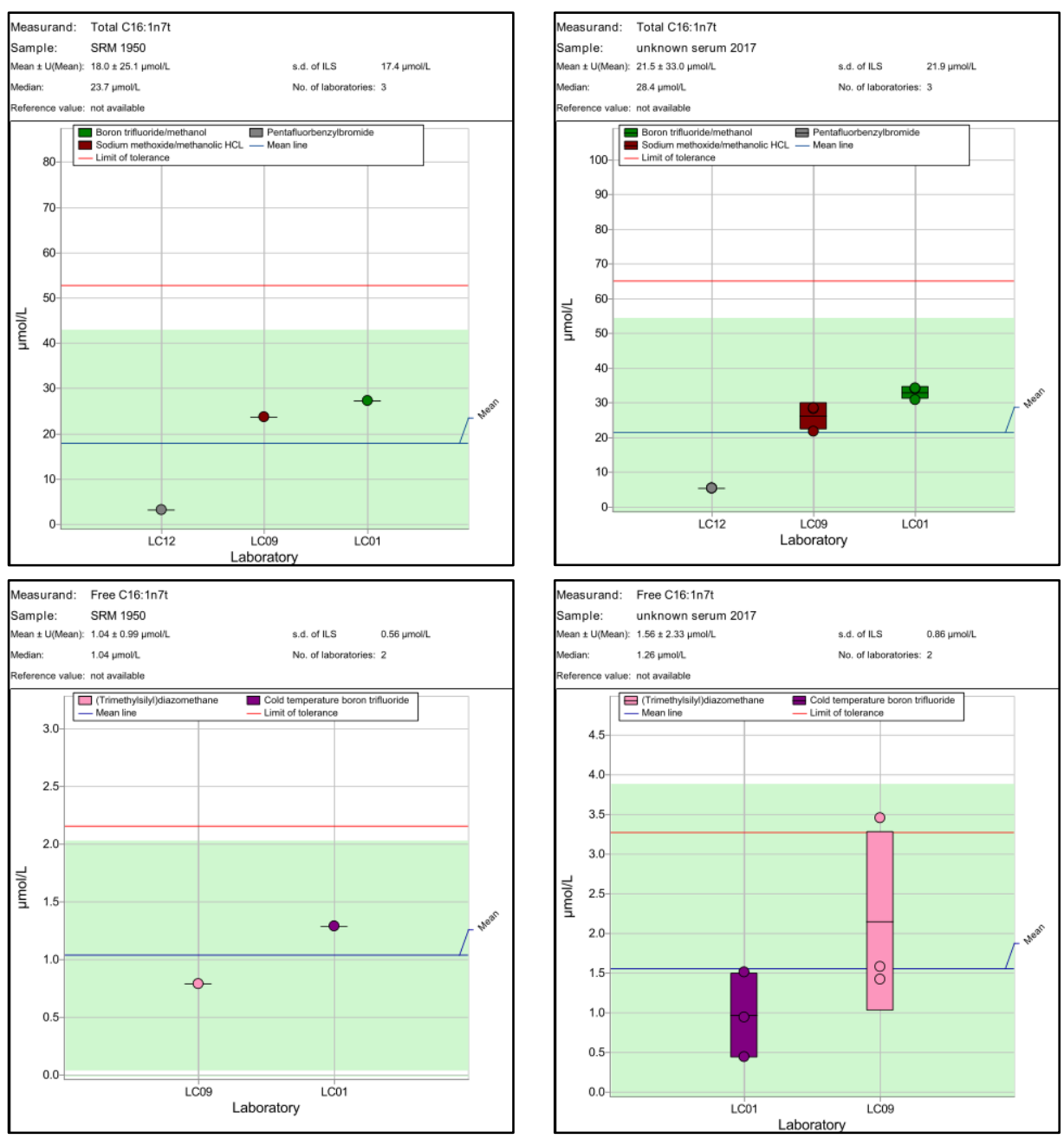
Table A-15: C17:0 Margaric acid, $\mu \mathrm{mol} / \mathrm{L}$

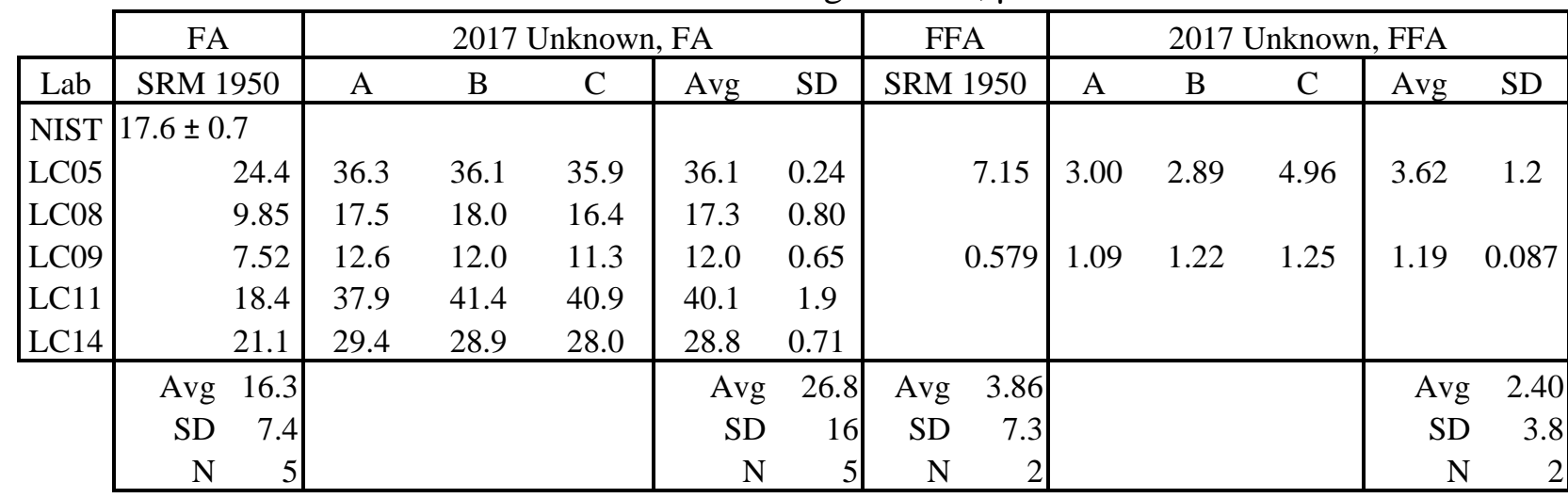
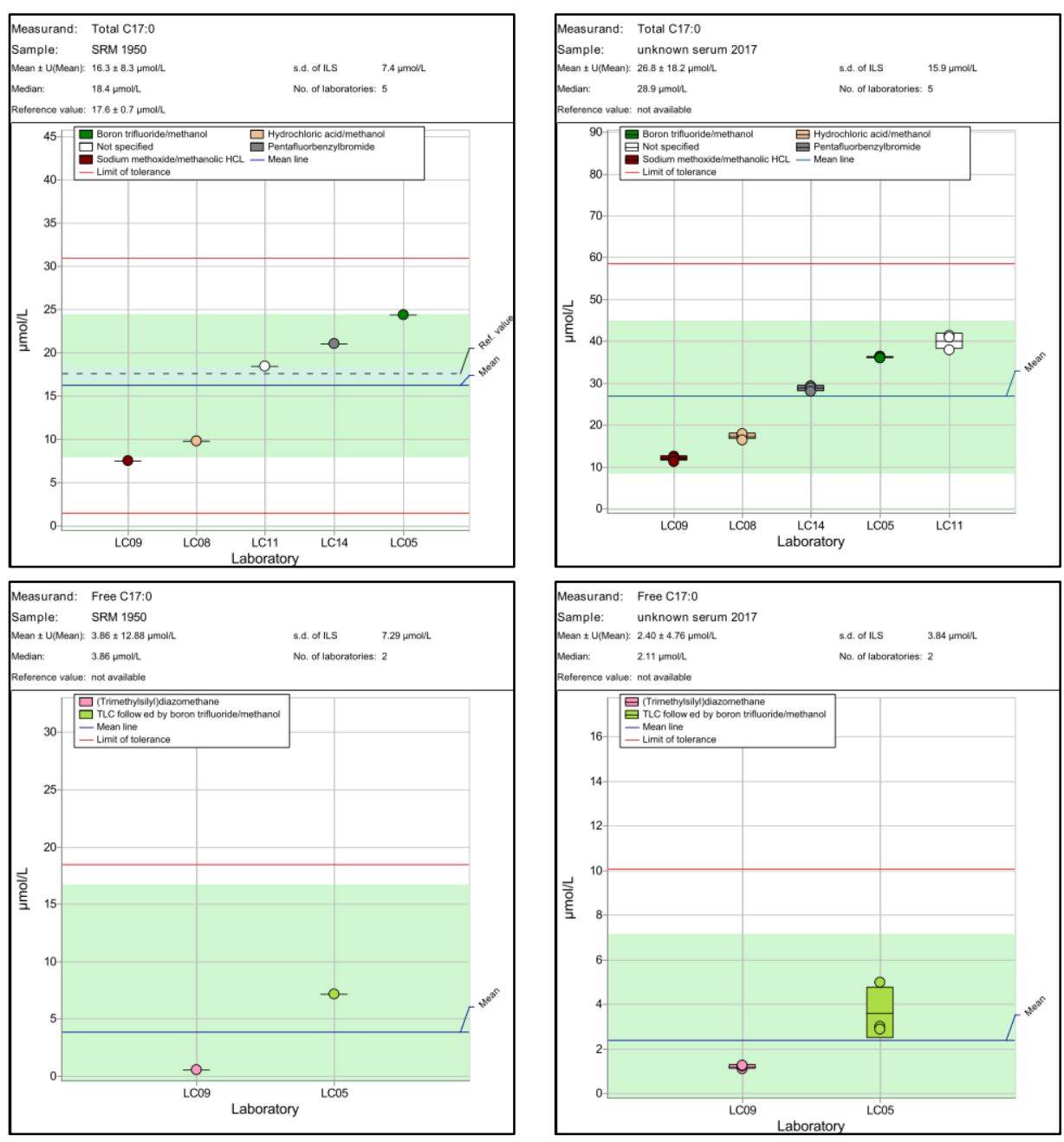
Table A-16: C18:0 Stearic acid, $\mu \mathrm{mol} / \mathrm{L}$

\begin{tabular}{|c|c|c|c|c|c|c|c|c|c|c|c|c|c|}
\hline & \multirow{2}{*}{$\frac{\text { FA }}{\text { SRM } 1950}$} & \multicolumn{5}{|c|}{2017 Unknown, FA } & \multirow{2}{*}{\multicolumn{2}{|c|}{$\frac{\text { FFA }}{\text { SRM } 1950}$}} & \multicolumn{5}{|c|}{2017 Unknown, FFA } \\
\hline Lab & & A & B & C & Avg & SD & & & A & B & $\mathrm{C}$ & Avg & SD \\
\hline NIST & $644 \pm 41$ & & & & & & & & & & & & \\
\hline LC01 & 698 & 858 & 915 & 906 & 893 & 31 & & 4.1 & 70.3 & 70.0 & 70.0 & 70.1 & 0.17 \\
\hline LC02 & 767 & 1055 & 1025 & 1026 & 1035 & 17 & & & & & & & \\
\hline LC04 & 548 & 713 & 762 & 745 & 740 & 25 & & & & & & & \\
\hline LC05 & 692 & 920 & 914 & 911 & 915 & 4.2 & & 5.5 & 17.5 & 24.5 & 26.5 & 22.9 & 4.7 \\
\hline LC06 & & & & & & & & 8.1 & 60.6 & 54.4 & 48.6 & 54.5 & 6.0 \\
\hline LC07 & 704 & 990 & 904 & 939 & 944 & 43 & & & & & & & \\
\hline LC08 & 440 & 566 & 592 & 559 & 572 & 17 & & & & & & & \\
\hline LC09 & 249 & 452 & 356 & 425 & 411 & 49 & & .35 & 36.2 & 33.1 & 34.2 & 34.5 & 1.55 \\
\hline LC10 & 736 & 884 & 906 & 945 & 912 & 30 & & & & & & & \\
\hline LC11 & 919 & 977 & 963 & 947 & 963 & 15 & & & & & & & \\
\hline LC12 & 639 & 863 & 871 & 870 & 868 & 4.5 & & & & & & & \\
\hline LC14 & 499 & 709 & 723 & 653 & 695 & 36.8 & & & & & & & \\
\hline & Avg 636 & & & & Avg & & Avg & 31.8 & & & & Avg & 45.5 \\
\hline & SD 157 & & & & SD & 169 & SD & 36 & & & & SD & 34 \\
\hline & 11 & & & & $\mathrm{~N}$ & 11 & $\mathrm{~N}$ & 4 & & & & $\mathrm{~N}$ & 4 \\
\hline
\end{tabular}
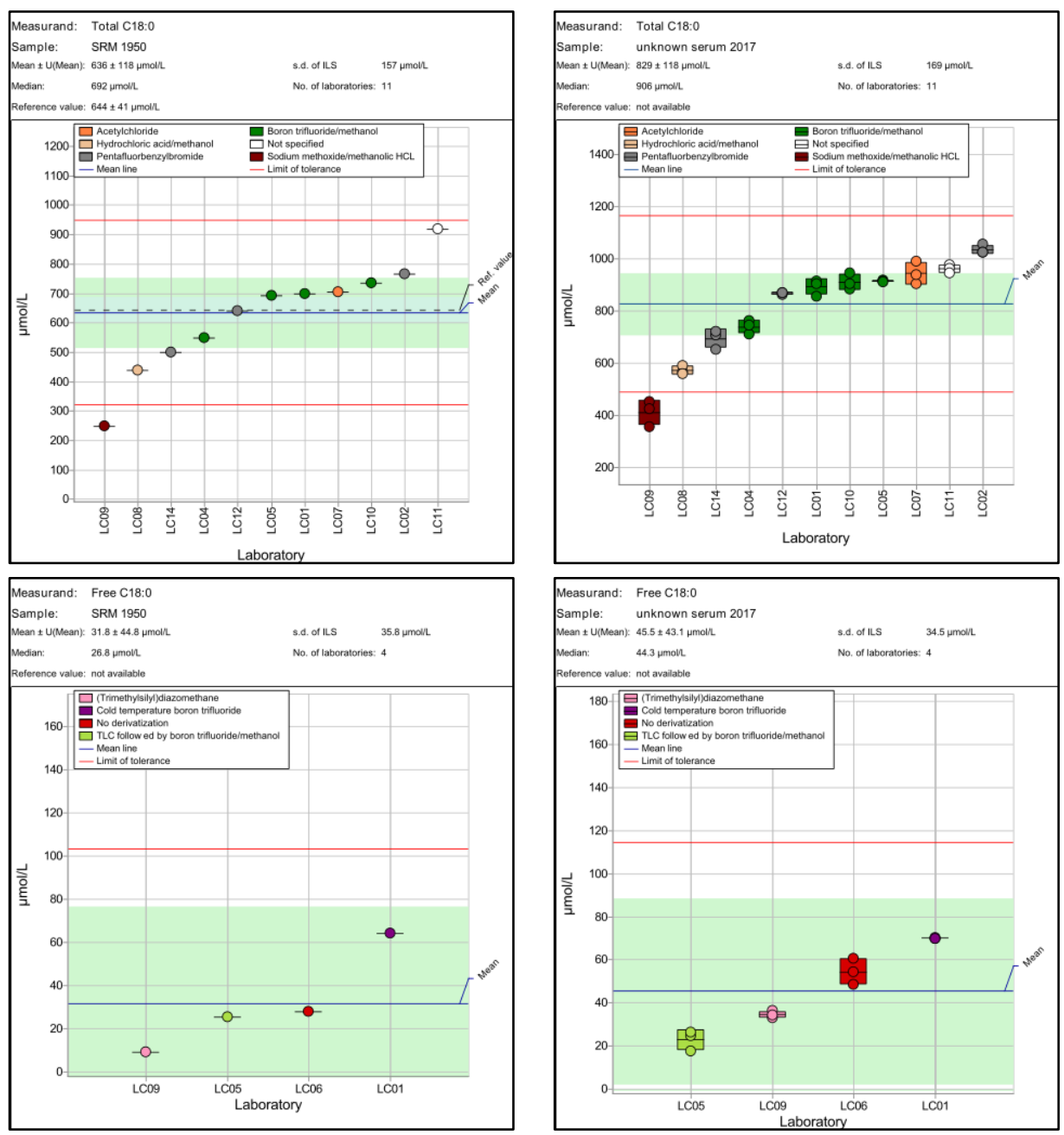
Table A-17: C18:1n7 cis-Vaccenic acid, $\mu \mathrm{mol} / \mathrm{L}$

\begin{tabular}{|c|c|c|c|c|c|c|c|c|c|c|c|c|c|}
\hline & FA & & 2017 & knov & & & FFA & & & 2017 & nknov & FFA & \\
\hline Lab & SRM 1950 & A & B & $\mathrm{C}$ & Avg & SD & SRM 19 & & A & B & $\mathrm{C}$ & Avg & SD \\
\hline NIST & $136 \pm 3$ & & & & & & \multirow{9}{*}{\multicolumn{2}{|c|}{$\begin{array}{l}10.8 \\
21.9\end{array}$}} & \multirow{9}{*}{$\begin{array}{c}11.3 \\
16.4 \\
0.306\end{array}$} & \multirow{9}{*}{$\begin{array}{l}11.2 \\
17.5 \\
2.64\end{array}$} & \multirow{9}{*}{$\begin{array}{l}11.1 \\
17.3 \\
2.92\end{array}$} & \multirow{9}{*}{$\begin{array}{l}11.2 \\
17.1 \\
1.95\end{array}$} & \multirow{9}{*}{$\begin{array}{c}0.076 \\
0.57 \\
1.4\end{array}$} \\
\hline LC02 & & 456 & 484 & 441 & 460 & 22 & & & & & & & \\
\hline LC04 & 131 & 159 & 179 & 175 & 171 & 11 & & & & & & & \\
\hline LC05 & \multirow[t]{2}{*}{199} & \multirow[t]{2}{*}{251} & \multirow[t]{2}{*}{248} & \multirow[t]{2}{*}{247} & \multirow[t]{2}{*}{249} & \multirow[t]{2}{*}{2.3} & & & & & & & \\
\hline LC06 & & & & & & & & & & & & & \\
\hline LC09 & 166 & 174 & 177 & 174 & 175 & 1.6 & & & & & & & \\
\hline LC10 & 186 & 148 & 148 & 153 & 150 & 2.8 & & & & & & & \\
\hline LC12 & 140 & 161 & 162 & 161 & 161 & 0.79 & & & & & & & \\
\hline LC14 & 126 & 129 & 122 & 116 & 123 & 6.6 & & & & & & & \\
\hline & Avg 158 & & & & Avg & 171 & Avg & 16.4 & & & & Avg & 10.1 \\
\hline & SD & & & & $\mathrm{SD}$ & 57 & SD & 12 & & & & SD & 14 \\
\hline & $\mathrm{N}$ & & & & $\mathrm{N}$ & 7 & $\mathrm{~N}$ & 2 & & & & $\mathrm{~N}$ & 3 \\
\hline
\end{tabular}
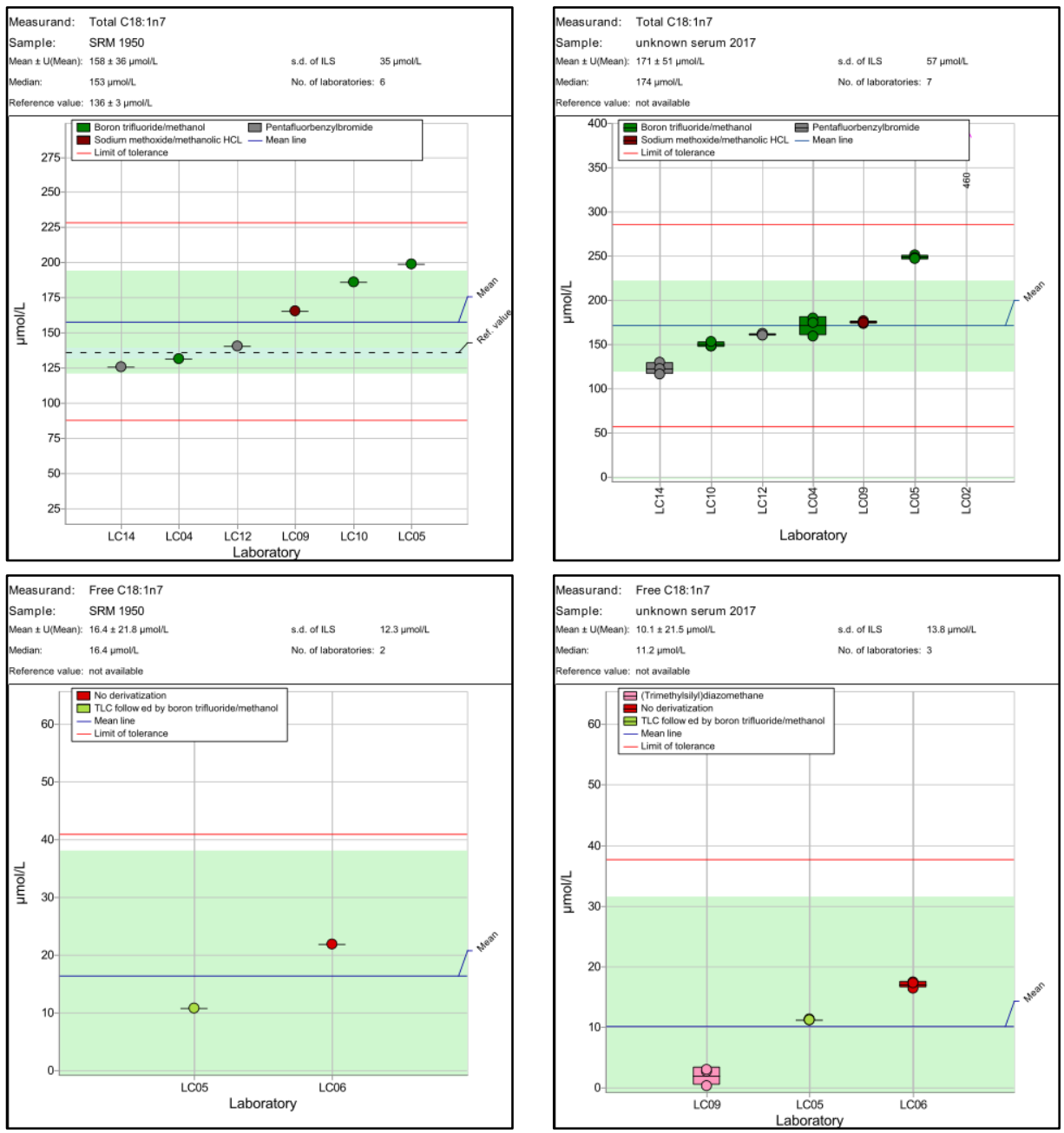
Table A-18: C18:1n9 Oleic acid, $\mu \mathrm{mol} / \mathrm{L}$

\begin{tabular}{|c|c|c|c|c|c|c|c|c|c|c|c|c|c|}
\hline \multirow{3}{*}{ Lab } & FA & \multicolumn{5}{|c|}{2017 Unknown, FA } & \multicolumn{2}{|c|}{ FFA } & \multicolumn{5}{|c|}{2017 Unknown, FFA } \\
\hline & SRM 1950 & A & B & $\mathrm{C}$ & Avg & SD & SRM & 950 & A & B & $\mathrm{C}$ & Avg & SD \\
\hline & $1614 \pm 154$ & & & & & & & & & & & & \\
\hline LC01 & 1840 & 2173 & 2316 & 2300 & 2263 & 79 & & 171 & 139 & 144 & 139 & 141 & 2.8 \\
\hline LC02 & 1744 & 2295 & 2211 & 2262 & 2256 & 42 & & & & & & & \\
\hline LC04 & 1090 & 1338 & 1560 & 1467 & 1455 & 112 & & & & & & & \\
\hline LC05 & 1852 & 2459 & 2364 & 2346 & 2390 & 61 & & 125 & 119 & 120 & 120 & 120 & 0.44 \\
\hline LC06 & & & & & & & & 157 & 114 & 136 & 127 & 126 & 11.1 \\
\hline LC07 & 1713 & 2348 & 2176 & 2261 & 2262 & 86 & & & & & & & \\
\hline LC08 & 998 & 631 & 610 & 560 & 601 & 37 & & & & & & & \\
\hline LC09 & 2688 & 3253 & 3310 & 3196 & 3253 & 57 & & 144 & 152 & 149 & 140 & 147 & 6.3 \\
\hline LC10 & 1971 & 2348 & 2390 & 2405 & 2381 & 29 & & & & & & & \\
\hline LC11 & 1884 & 1769 & 1764 & 1795 & 1776 & 17 & & & & & & & \\
\hline LC12 & 1660 & 2160 & 2170 & 2160 & 2163 & 5.8 & & & & & & & \\
\hline LC14 & 1670 & 1868 & 1759 & 1665 & 1764 & 102 & & & & & & & \\
\hline & Avg 1721 & & & & Avg & 2103 & Avg & & & & & Avg & 133 \\
\hline & SD 268 & & & & SD & 430 & SD & & & & & SD & 18 \\
\hline & $\mathrm{N} \quad 11$ & & & & $\mathrm{~N}$ & 11 & $\mathrm{~N}$ & 4 & & & & $\mathrm{~N}$ & 4 \\
\hline
\end{tabular}
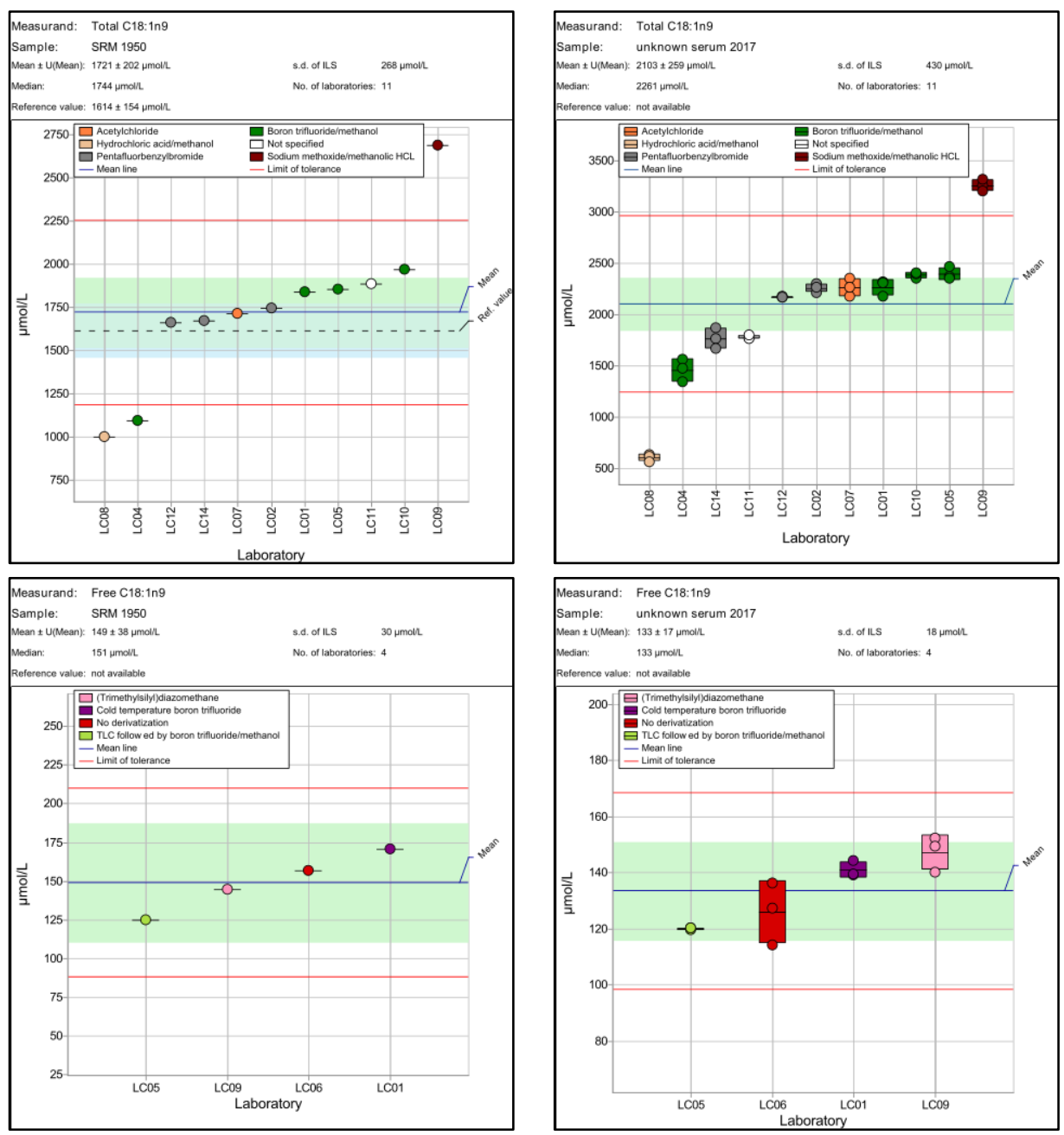
Table A-19: C18:1n9t Elaidic acid, $\mu \mathrm{mol} / \mathrm{L}$

\begin{tabular}{|c|c|c|c|c|c|c|c|c|c|c|c|c|}
\hline & FA & \multicolumn{5}{|c|}{2017 Unknown, FA } & FFA & \multicolumn{5}{|c|}{2017 Unknown, FFA } \\
\hline Lab & SRM 1950 & A & B & $\mathrm{C}$ & Avg & SD & SRM 1950 & A & B & $\mathrm{C}$ & Avg & SD \\
\hline LC01 & 113 & 156 & 171 & 182 & 170 & 13.0 & \multirow[t]{4}{*}{20.1} & \multirow[t]{4}{*}{15.0} & \multirow[t]{4}{*}{20.4} & \multirow[t]{4}{*}{16.2} & \multirow[t]{4}{*}{17.2} & \multirow[t]{4}{*}{2.8} \\
\hline LC08 & 3.15 & 0.500 & 2.12 & 0.450 & 1.02 & 0.95 & & & & & & \\
\hline LC11 & 80.5 & 108 & 102 & 110 & 107 & 4.3 & & & & & & \\
\hline LC12 & 18.5 & 30.8 & 30.8 & 31.0 & 30.9 & 0.10 & & & & & & \\
\hline & Avg 53.8 & & & & Avg & 77.0 & & & & & & \\
\hline & SD & & & & SD & 140 & & & & & & \\
\hline & $\mathrm{N}$ & & & & $\mathrm{N}$ & 4 & $\mathrm{~N}$ & & & & & \\
\hline
\end{tabular}
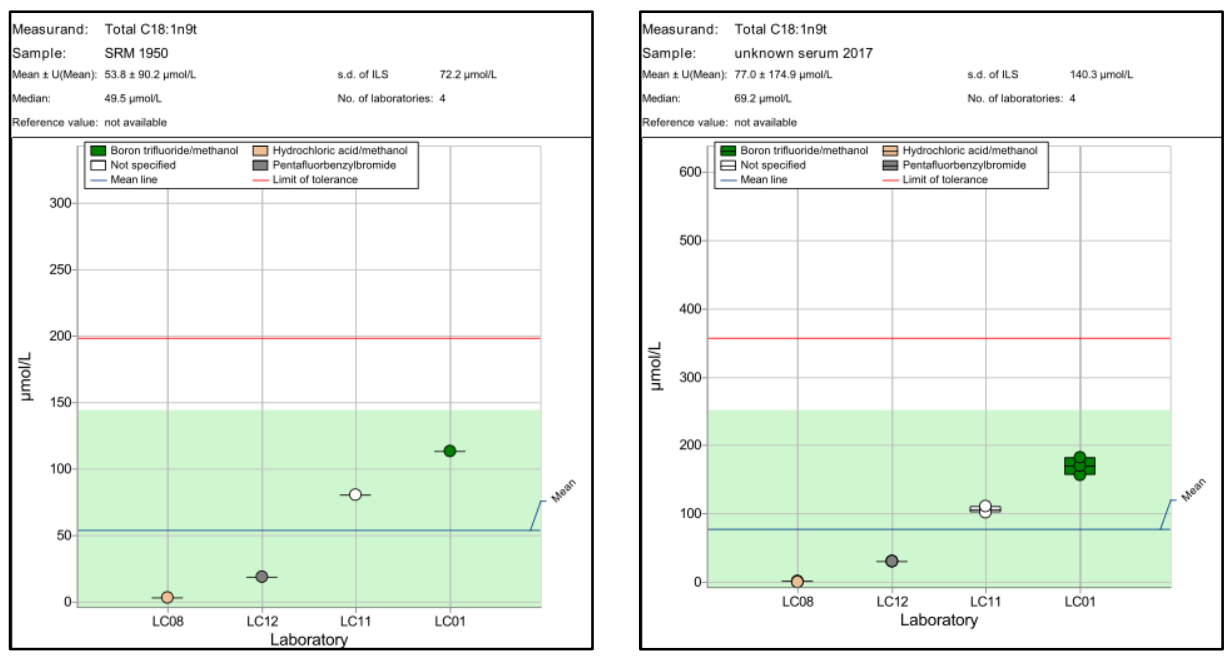
Table A-20: C18:2n6 Linoleic acid, $\mu \mathrm{mol} / \mathrm{L}$

\begin{tabular}{|c|c|c|c|c|c|c|c|c|c|c|c|c|c|}
\hline & \multirow{2}{*}{$\frac{\text { FA }}{\text { SRM } 1950}$} & \multicolumn{5}{|c|}{2017 Unknown, FA } & \multicolumn{2}{|l|}{ FFA } & \multicolumn{5}{|c|}{2017 Unknown, FFA } \\
\hline Lab & & A & B & C & Avg & SD & SRM 19 & & A & B & $\mathrm{C}$ & Avg & SD \\
\hline NIST & $2838 \pm 143$ & & & & & & & & & & & & \\
\hline LC01 & 2903 & 3639 & 3879 & 3858 & 3792 & 133 & & 4.1 & 113 & 113 & 113 & 113 & 0.082 \\
\hline LC02 & 2629 & 3590 & 3482 & 3651 & 3574 & 86 & & & & & & & \\
\hline LC04 & 1704 & 2209 & 2561 & 2403 & 2391 & 176 & & & & & & & \\
\hline LC05 & 2896 & 3913 & 3888 & 3859 & 3886 & 27 & & 1.6 & 92.8 & 92.5 & 92.7 & 92.7 & 0.16 \\
\hline LC06 & & & & & & & & 1.3 & 108 & 150 & 124 & 128 & 21 \\
\hline LC07 & 2830 & 4143 & 3842 & 3997 & 3994 & 151 & & & & & & & \\
\hline LC08 & 1393 & 1529 & 1352 & 1267 & 1383 & 134 & & & & & & & \\
\hline LC09 & 4049 & 5115 & 5196 & 5023 & 5111 & 86 & & 4.6 & 107 & 104 & 94.6 & 102 & 6.5 \\
\hline LC10 & 2970 & 3885 & 3950 & 3980 & 3938 & 48 & & & & & & & \\
\hline LC11 & 2835 & 2714 & 2713 & 2734 & 2720 & 12 & & & & & & & \\
\hline LC12 & 2660 & 3700 & 3700 & 3690 & 3697 & 5.8 & & & & & & & \\
\hline LC14 & 2562 & 3082 & 2888 & 2741 & 2904 & 171 & & & & & & & \\
\hline & Avg 2712 & & & & Avg & 3433 & & 70.4 & & & & Avg & 109 \\
\hline & SD 380 & & & & SD & 726 & SD & 43 & & & & SD & 20 \\
\hline & $\mathrm{N}$ & & & & $\mathrm{N}$ & 11 & $\mathrm{~N}$ & 4 & & & & $\mathrm{~N}$ & 4 \\
\hline
\end{tabular}
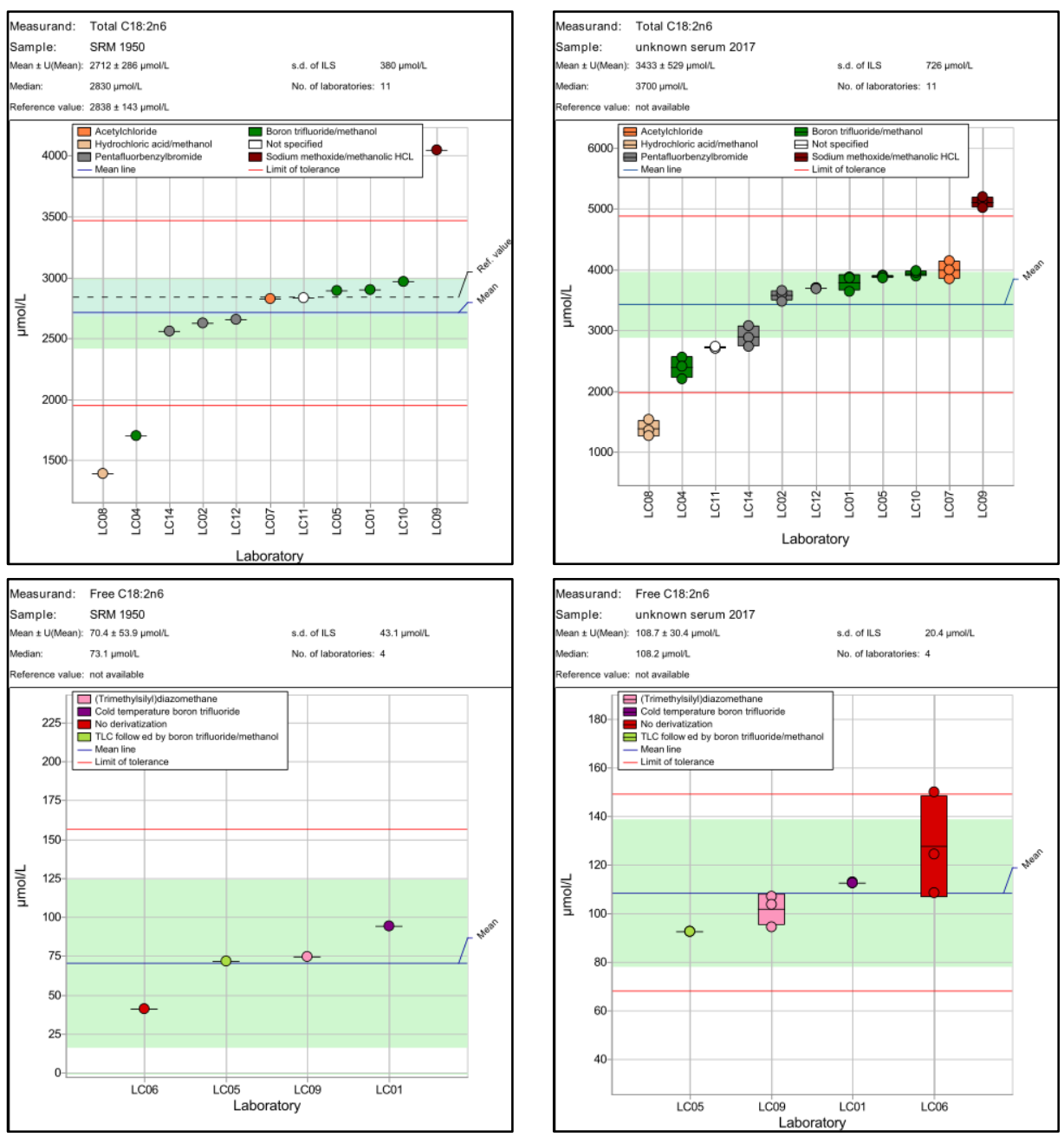
Table A-21: C18:2n6t,9t Linoelaidic acid, $\mu \mathrm{mol} / \mathrm{L}$

\begin{tabular}{|c|c|c|c|c|c|c|c|c|c|c|c|c|}
\hline & FA & \multicolumn{5}{|c|}{2017 Unknown, FA } & FFA & \multicolumn{5}{|c|}{2017 Unknown, FFA } \\
\hline Lab & SRM 1950 & A & B & C & Avg & SD & SRM 1950 & A & B & $\mathrm{C}$ & Avg & SD \\
\hline LC01 & 59.0 & 88.6 & 77.0 & 81.4 & 82.3 & 5.9 & \multirow[t]{5}{*}{8.95} & \multirow[t]{5}{*}{9.71} & \multirow[t]{5}{*}{8.97} & \multirow{5}{*}{5.74} & \multirow[t]{5}{*}{8.14} & \multirow[t]{5}{*}{2.1} \\
\hline LC08 & 58.3 & 55.4 & 56.4 & 57.5 & 56.4 & 1.0 & & & & & & \\
\hline LC10 & $<\mathrm{LOD}$ & 20.8 & 20.3 & $<\mathrm{LOD}$ & 20.5 & 0.30 & & & & & & \\
\hline LC11 & 228 & 222 & 230 & 234 & 229 & 6.3 & & & & & & \\
\hline LC12 & 1.60 & 2.08 & 1.88 & 2.09 & 2.01 & 0.12 & & & & & & \\
\hline & Avg 86.7 & & & & Avg & 70.2 & & & & & & \\
\hline & SD 126 & & & & SD & 80 & & & & & & \\
\hline & $\mathrm{N} \quad 4$ & & & & $\mathrm{~N}$ & 5 & $\mathrm{~N}$ & & & & & \\
\hline
\end{tabular}
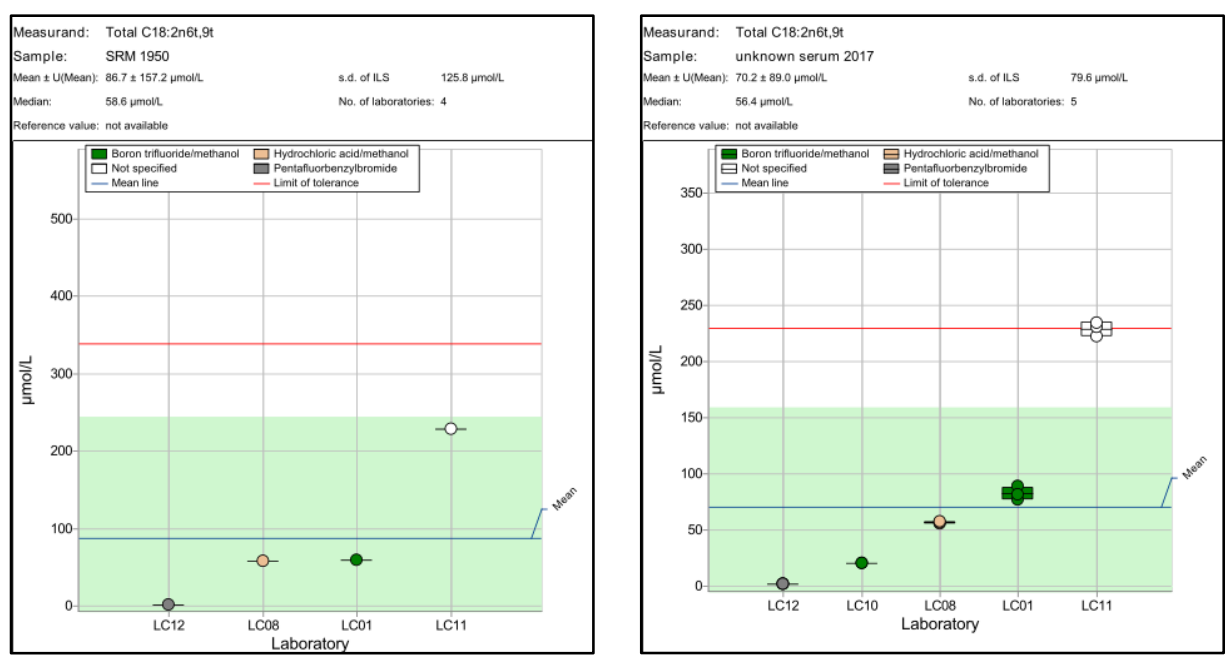
Table A-22: C18:3n3 $\alpha$-Linolenic acid, $\mu \mathrm{mol} / \mathrm{L}$

\begin{tabular}{|c|c|c|c|c|c|c|c|c|c|c|c|c|c|}
\hline & \multirow{2}{*}{$\frac{\text { FA }}{\text { SRM } 1950}$} & \multicolumn{5}{|c|}{2017 Unknown, FA } & \multicolumn{2}{|c|}{ FFA } & \multicolumn{5}{|c|}{2017 Unknown, FFA } \\
\hline Lab & & A & B & $\mathrm{C}$ & Avg & SD & SRM & 1950 & A & B & $\mathrm{C}$ & Avg & SD \\
\hline NIST & $54.6 \pm 3.6$ & & & & & & & & & & & & \\
\hline LC01 & 53.1 & 60.6 & 63.6 & 62.8 & 62.3 & 1.6 & & 4.79 & 3.72 & 3.71 & 4.58 & 4.00 & 0.50 \\
\hline LC02 & 54.0 & 79.0 & 73.0 & 76.0 & 76.0 & 3.0 & & & & & & & \\
\hline LC04 & 33.0 & 40.2 & 45.4 & 42.4 & 42.7 & 2.6 & & & & & & & \\
\hline LC05 & 51.8 & 64.9 & 64.7 & 63.7 & 64.4 & 0.65 & & 2.97 & 2.93 & 2.90 & 2.72 & 2.85 & 0.11 \\
\hline LC07 & 58.2 & 79.0 & 73.3 & 75.8 & 76.0 & 2.9 & & & & & & & \\
\hline LC08 & 17.4 & 18.9 & 15.4 & 13.9 & 16.0 & 2.6 & & & & & & & \\
\hline LC09 & 28.9 & 28.5 & 28.9 & 27.5 & 28.3 & 0.71 & & & & & & & \\
\hline LC10 & 57.4 & 66.0 & 67.1 & 68.1 & 67.1 & 1.1 & & & & & & & \\
\hline LC11 & 46.7 & 49.0 & 53.5 & 57.1 & 53.2 & 4.0 & & & & & & & \\
\hline LC12 & 45.0 & 56.8 & 57.9 & 57.2 & 57.3 & 0.56 & & & & & & & \\
\hline LC14 & 46.5 & 70.3 & 70.2 & 52.8 & 64.5 & 10.1 & & & & & & & \\
\hline & Avg 45.7 & & & & Avg & 56.3 & Avg & 3.88 & & & & Avg & 3.43 \\
\hline & SD 12 & & & & SD & 20 & SD & 2.0 & & & & SD & 1.8 \\
\hline & $\mathrm{N}$ & & & & $\mathrm{N}$ & 11 & $\mathrm{~N}$ & 2 & & & & $\mathrm{~N}$ & 2 \\
\hline
\end{tabular}
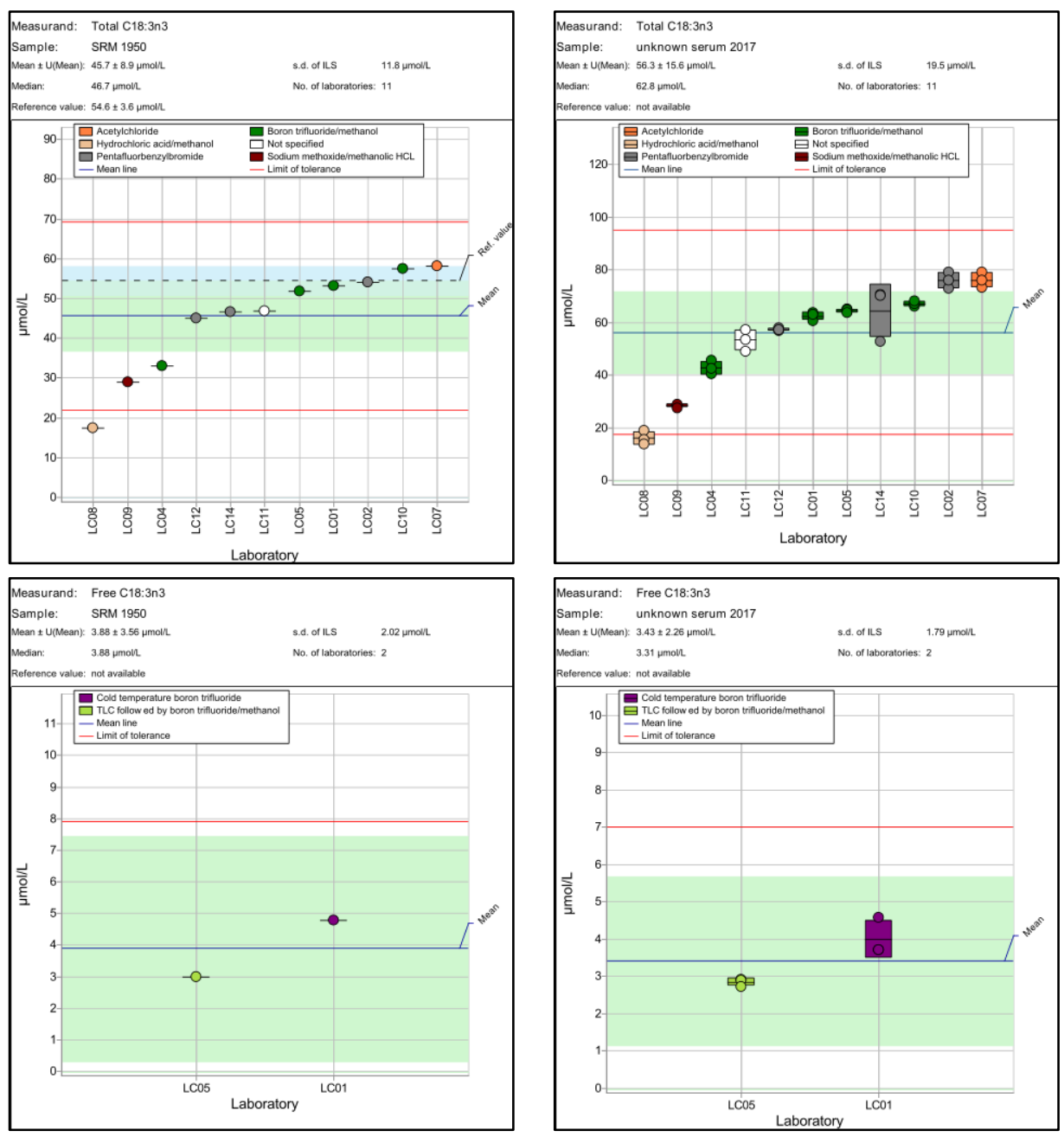
Table A-23: C18:3n6 $\psi$-Linolenic acid, $\mu \mathrm{mol} / \mathrm{L}$

\begin{tabular}{|c|c|c|c|c|c|c|c|c|c|c|c|c|}
\hline & \multirow{2}{*}{$\frac{\text { FA }}{\text { SRM } 1950}$} & \multicolumn{5}{|c|}{2017 Unknown, FA } & \multirow{2}{*}{$\frac{\text { FFA }}{\text { SRM } 1950}$} & \multicolumn{5}{|c|}{2017 Unknown, FFA } \\
\hline Lab & & A & $\mathrm{B}$ & $\mathrm{C}$ & Avg & SD & & A & $\mathrm{B}$ & $\mathrm{C}$ & Avg & SD \\
\hline NIST & $39.9 \pm 8.5$ & & & & & & & & & & & \\
\hline LC01 & 48.7 & 50.7 & 55.5 & 49.9 & 52.0 & 3.0 & 0.992 & 0.651 & 0.672 & 0.583 & 0.635 & 0.047 \\
\hline LC02 & & 60.0 & 59.0 & 61.0 & 60.0 & 1.0 & & & & & & \\
\hline LC04 & 28.2 & 24.9 & 29.8 & 27.6 & 27.5 & 2.4 & & & & & & \\
\hline LC05 & 51.3 & 49.6 & 48.6 & 48.5 & 48.9 & 0.62 & 1.02 & 1.09 & 1.14 & 0.900 & 1.04 & 0.13 \\
\hline LC07 & 56.7 & 63.9 & 56.4 & 57.5 & 59.3 & 4.1 & & & & & & \\
\hline LC08 & 29.5 & 17.9 & 13.7 & 13.8 & 15.1 & 2.4 & & & & & & \\
\hline LC09 & 44.5 & 28.9 & 30.2 & 29.7 & 29.6 & 0.66 & 0.984 & 0.764 & 0.838 & 1.13 & 0.911 & 0.19 \\
\hline LC10 & 56.6 & 49.0 & 49.6 & 48.6 & 49.1 & 0.50 & & & & & & \\
\hline LC11 & 2.50 & 2.46 & 1.23 & 2.08 & 1.92 & 0.63 & & & & & & \\
\hline LC12 & 48.1 & 44.4 & 45.6 & 45.0 & 45.0 & 0.61 & & & & & & \\
\hline LC14 & 48.2 & 39.3 & 36.9 & 34.7 & 37.0 & 2 & & & & & & \\
\hline & Avg 45.0 & & & & Avg & 39.1 & Avg 0.997 & & & & Avg & 0.863 \\
\hline & SD 11 & & & & SD & 22 & SD 0.025 & & & & SD & 0.38 \\
\hline & 10 & & & & $\mathrm{~N}$ & 11 & $\mathrm{~N}$ & & & & $\mathrm{~N}$ & 3 \\
\hline
\end{tabular}
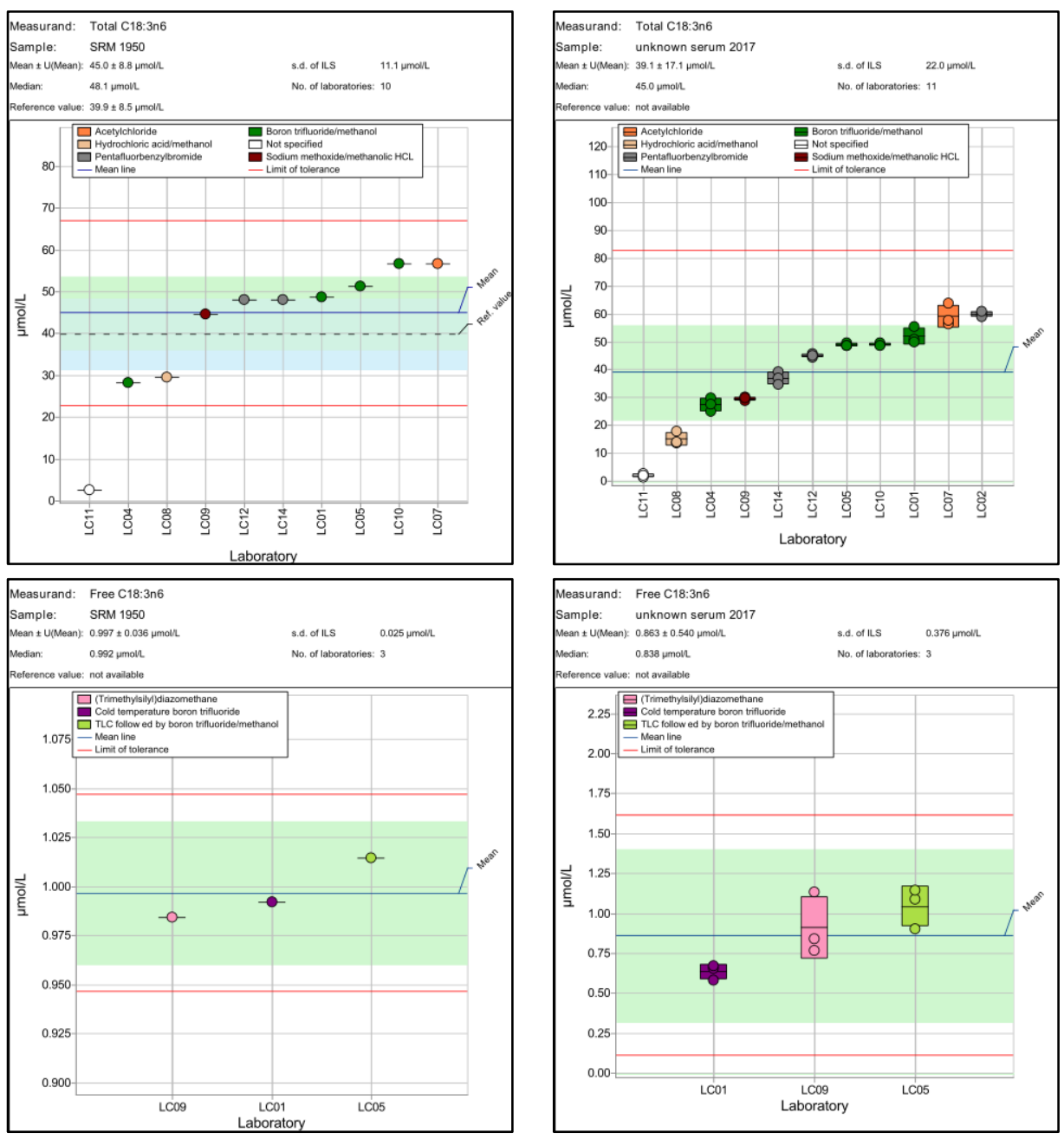
Table A-24: C18:4n3 Stearidonic acid, $\mu \mathrm{mol} / \mathrm{L}$

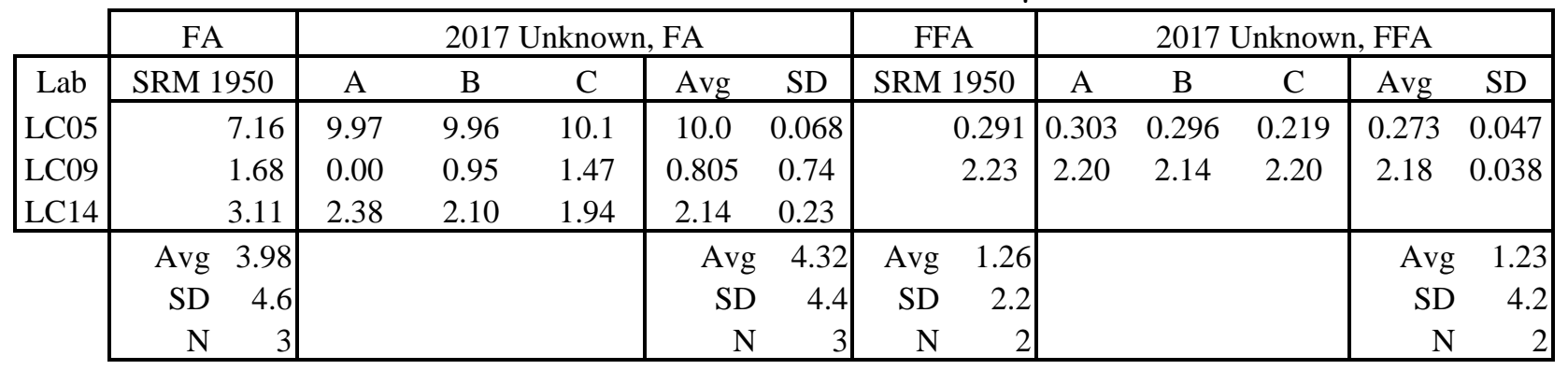
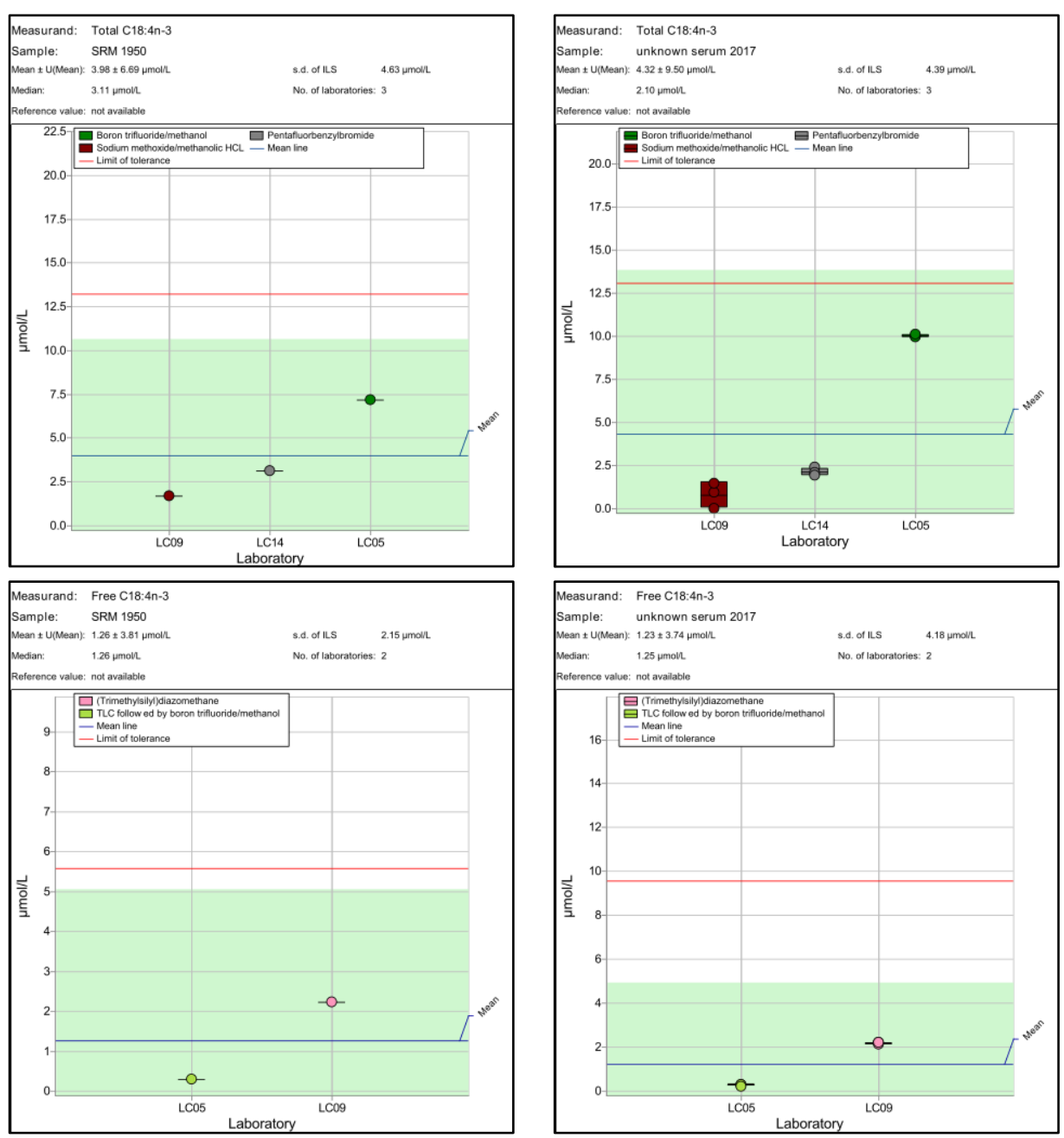
Table A-25: C19:0 Nonadecanoic acid, $\mu \mathrm{mol} / \mathrm{L}$

\begin{tabular}{|c|c|c|c|c|c|c|c|c|c|c|c|c|}
\hline & FA & \multicolumn{5}{|c|}{2017 Unknown, FA } & FFA & \multicolumn{5}{|c|}{2017 Unknown, FFA } \\
\hline Lab & SRM 1950 & A & B & $\mathrm{C}$ & Avg & SD & SRM 1950 & A & B & $\mathrm{C}$ & Avg & SD \\
\hline LC05 & 3.67 & 5.43 & 4.72 & 4.74 & 4.96 & 0.40 & 0.387 & 0.404 & 0.450 & 0.242 & 0.365 & 0.11 \\
\hline LC09 & 0.423 & 0.659 & 0.671 & 0.825 & 0.718 & 0.093 & & & & & & \\
\hline & $\begin{array}{rr}\text { Avg } & 2.05 \\
\text { SD } & 3.6 \\
\mathrm{~N} & 2\end{array}$ & & & & $\begin{array}{r}\text { Avg } \\
\text { SD } \\
\mathrm{N}\end{array}$ & $\begin{array}{r}2.84 \\
8.9 \\
2\end{array}$ & $\mathrm{~N}$ & & & & $\mathrm{~N}$ & 1 \\
\hline
\end{tabular}
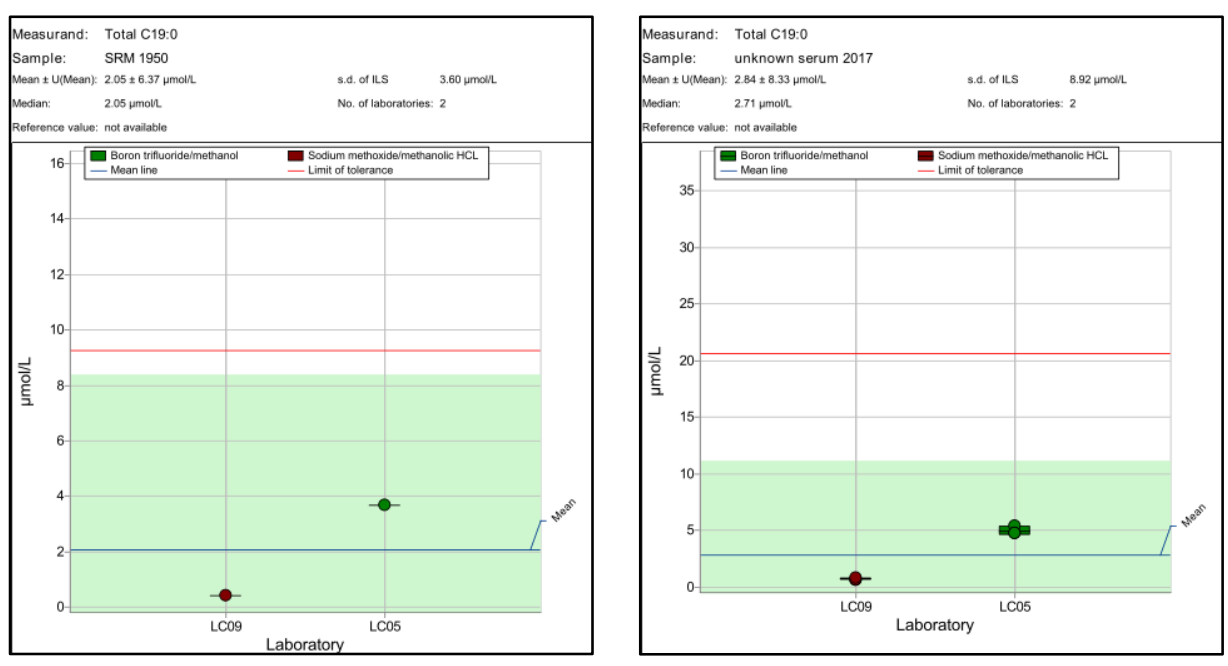
Table A-26: C20:0 Arachidic acid, $\mu \mathrm{mol} / \mathrm{L}$

\begin{tabular}{|c|c|c|c|c|c|c|c|c|c|c|c|c|c|}
\hline \multirow[b]{2}{*}{ Lab } & FA & \multicolumn{5}{|c|}{2017 Unknown, FA } & \multicolumn{2}{|c|}{ FFA } & \multicolumn{5}{|c|}{2017 Unknown, FFA } \\
\hline & SRM 1950 & A & $\mathrm{B}$ & $\mathrm{C}$ & Avg & SD & SRM & 1950 & A & B & $\mathrm{C}$ & Avg & SD \\
\hline NIST & $18.0 \pm 0.5$ & & & & & & & & & & & & \\
\hline LC01 & 14.14 & 11.0 & 13.2 & 14.3 & 12.8 & 1.6 & & 0.512 & 0.395 & 0.324 & 0.397 & 0.372 & 0.042 \\
\hline LC02 & 25.0 & 36.0 & 35.0 & 35.0 & 35.3 & 0.58 & & & & & & & \\
\hline LC04 & 22.7 & 32.2 & 36.1 & 37.4 & 35.3 & 2.7 & & & & & & & \\
\hline LC05 & 16.1 & 23.8 & 23.5 & 24.1 & 23.8 & 0.30 & & 1.63 & 1.43 & 1.53 & 1.56 & 1.51 & 0.071 \\
\hline LC07 & 18.6 & 29.4 & 26.9 & 28.1 & 28.1 & 1.3 & & & & & & & \\
\hline LC08 & 34.3 & 13.2 & 13.0 & 13.0 & 13.0 & 0.12 & & & & & & & \\
\hline LC11 & 21.4 & 27.1 & 33.4 & 33.1 & 31.2 & 3.6 & & & & & & & \\
\hline LC12 & 16.5 & 25.7 & 25.6 & 25.6 & 25.6 & 0.087 & & & & & & & \\
\hline LC14 & 17.2 & 23.8 & 23.2 & 23.7 & 23.5 & 0.32 & & & & & & & \\
\hline & Avg 20.0 & & & & Avg & 25.4 & Avg & 1.07 & & & & Avg & 0.939 \\
\hline & SD 5.4 & & & & SD & 8.3 & SD & 1.2 & & & & $\mathrm{SD}$ & 2.4 \\
\hline & $\mathrm{N}$ & & & & $\mathrm{N}$ & 9 & $\mathrm{~N}$ & 2 & & & & $\mathrm{~N}$ & 2 \\
\hline
\end{tabular}
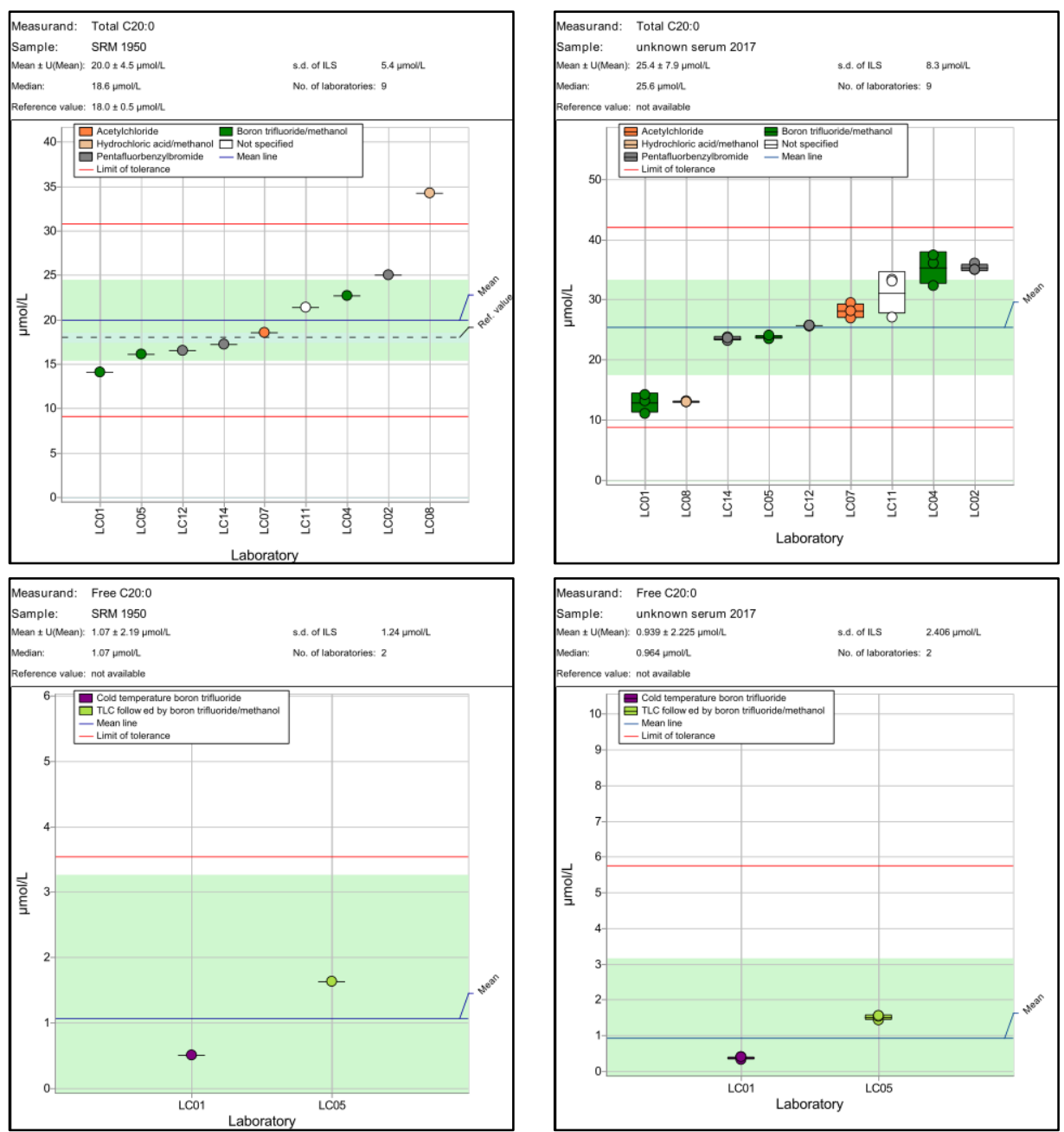
Table A-27: C20:1n9 11-Eicosenoic acid, $\mu \mathrm{mol} / \mathrm{L}$

\begin{tabular}{|c|c|c|c|c|c|c|c|c|c|c|c|c|c|}
\hline & \multirow{2}{*}{$\frac{\text { FA }}{\text { SRM } 1950}$} & \multicolumn{5}{|c|}{2017 Unknown, FA } & \multirow{2}{*}{\multicolumn{2}{|c|}{$\begin{array}{c}\text { FFA } \\
\text { SRM } 1950\end{array}$}} & \multicolumn{5}{|c|}{2017 Unknown, FFA } \\
\hline Lab & & A & $\mathrm{B}$ & $\mathrm{C}$ & Avg & SD & & & A & B & C & Avg & SD \\
\hline NIST & $11.5 \pm 0.5$ & & & & & & & & & & & & \\
\hline LC01 & 12.4 & 17.7 & 16.7 & 18.3 & 17.6 & 0.82 & & .11 & 0.856 & 0.655 & 0.730 & 0.747 & 0.10 \\
\hline LC04 & 6.98 & 8.26 & 8.96 & 8.77 & 8.67 & 0.36 & & & & & & & \\
\hline LC05 & 11.5 & 14.3 & 14.2 & 14.1 & 14.2 & 0.10 & & 17 & 1.12 & 1.37 & 1.13 & 1.21 & 0.14 \\
\hline LC08 & 6.99 & 5.80 & 4.12 & 3.72 & 4.55 & 1.1 & & & & & & & \\
\hline LC09 & 4.48 & 3.74 & 3.99 & 4.94 & 4.22 & 0.63 & & & & & & & \\
\hline LC10 & $<\mathrm{LOD}$ & 11.6 & $<$ LOD & $<\mathrm{LOD}$ & 11.6 & & & & & & & & \\
\hline LC11 & 16.8 & 17.9 & 16.2 & 17.1 & 17.1 & 0.86 & & & & & & & \\
\hline LC12 & 10.1 & 12.8 & 12.9 & 12.9 & 12.9 & 0.054 & & & & & & & \\
\hline LC14 & 10.3 & 11.3 & 11.5 & 10.9 & 11.2 & 0.32 & & & & & & & \\
\hline & Avg & & & & Avg & 11.3 & & 1.14 & & & & Avg & 0.978 \\
\hline & SD & & & & SD & 6.0 & SD 0 . & .064 & & & & $\mathrm{SD}$ & 0.81 \\
\hline & $\mathrm{N}$ & & & & $\mathrm{N}$ & 9 & $\mathrm{~N}$ & 2 & & & & $\mathrm{~N}$ & 2 \\
\hline
\end{tabular}
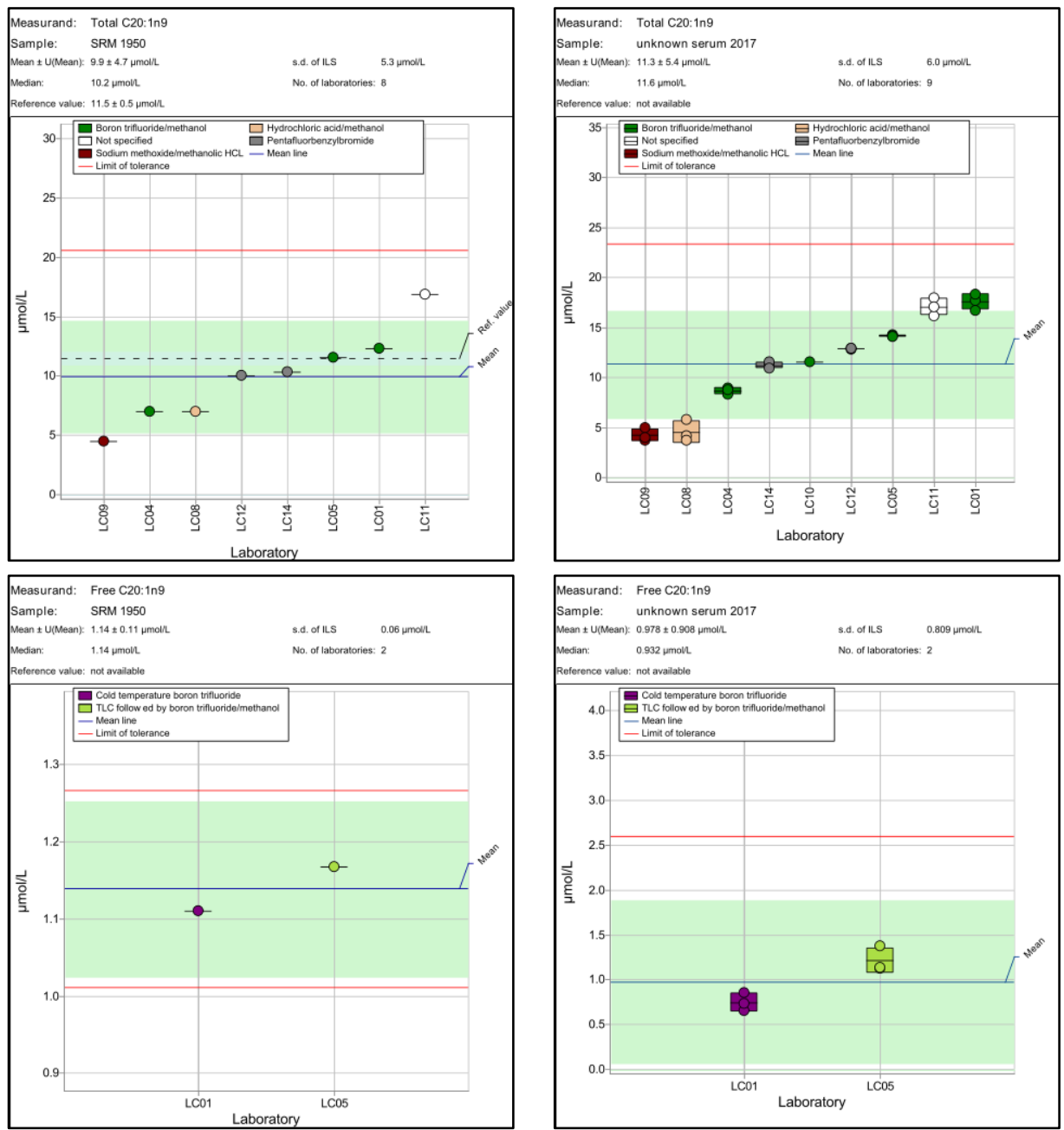
Table A-28: C20:2n6 11,14-Eicosadienoic acid, $\mu \mathrm{mol} / \mathrm{L}$

\begin{tabular}{|c|c|c|c|c|c|c|c|c|c|c|c|c|}
\hline & \multirow{2}{*}{$\frac{\text { FA }}{\text { SRM } 1950}$} & \multicolumn{5}{|c|}{2017 Unknown, FA } & FFA & \multicolumn{5}{|c|}{2017 Unknown, FFA } \\
\hline Lab & & A & $\mathrm{B}$ & $\mathrm{C}$ & Avg & SD & SRM 195 & A & B & C & Avg & SD \\
\hline NIST & $18.8 \pm 0.6$ & & & & & & & & & & & \\
\hline LC01 & 25.5 & 28.7 & 25.7 & 25.3 & 26.6 & 1.9 & 0.88 & 0.834 & 0.651 & 0.636 & 0.707 & 0.11 \\
\hline LC04 & 15.0 & 14.5 & 18.0 & 17.0 & 16.5 & 1.8 & & & & & & \\
\hline LC05 & 18.6 & 21.8 & 22.5 & 22.0 & 22.1 & 0.37 & $0.9 \mathrm{~s}$ & 0.855 & 0.925 & 0.842 & 0.874 & 0.045 \\
\hline LC08 & 8.96 & 10.4 & 10.1 & 11.5 & 10.6 & 0.72 & & & & & & \\
\hline LC09 & 8.27 & 7.69 & 8.28 & 7.75 & 7.91 & 0.33 & 0.27 & 0.234 & 0.312 & 0.247 & 0.264 & 0.042 \\
\hline LC10 & 30.6 & 30.9 & 30.7 & 31.1 & 30.9 & 0.19 & & & & & & \\
\hline LC11 & 18.2 & 24.9 & 25.9 & 25.5 & 25.4 & 0.49 & & & & & & \\
\hline LC12 & 17.6 & 21.5 & 21.7 & 22.0 & 21.7 & 0.25 & & & & & & \\
\hline LC14 & 18.2 & 19.9 & 19.5 & 19.5 & 19.6 & 0.26 & & & & & & \\
\hline & $\begin{array}{ll}\text { Avg } & 17.6\end{array}$ & & & & Avg & 20.2 & Avg 0.7 & & & & Avg & 0.615 \\
\hline & SD $\quad 7.0$ & & & & $\mathrm{SD}$ & 8.6 & SD 0. & & & & SD & 0.52 \\
\hline & $\mathrm{N}$ & & & & $\mathrm{N}$ & 9 & $\mathrm{~N}$ & 3 & & & $\mathrm{~N}$ & 3 \\
\hline
\end{tabular}
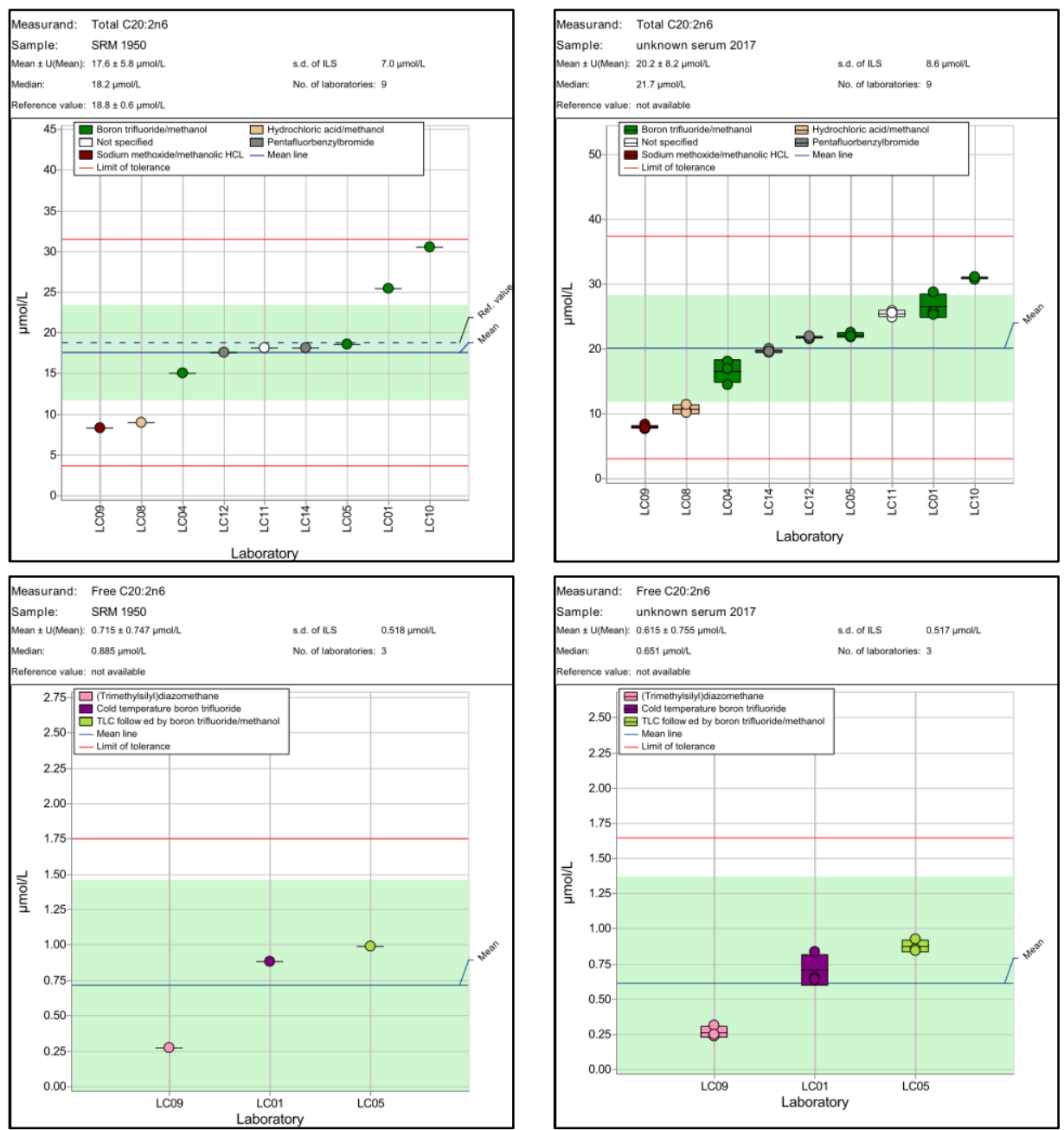
Table A-29: C20:3n3 11,14,17-Eicosatrienoic acid, $\mu \mathrm{mol} / \mathrm{L}$

\begin{tabular}{|c|c|c|c|c|c|c|c|c|c|c|c|}
\hline & FA & \multicolumn{5}{|c|}{2017 Unknown, FA } & FFA & \multicolumn{4}{|c|}{2017 Unknown, FFA } \\
\hline Lab & SRM 1950 & A & $\mathrm{B}$ & $\mathrm{C}$ & Avg & SD & SRM 1950 & A & $\mathrm{B}$ & $\mathrm{C}$ & Avg \\
\hline LC05 & 2.80 & 2.30 & 3.04 & 2.90 & 2.75 & 0.40 & 0.406 & 0.350 & 0.402 & 0.375 & $0.376 \quad 0.026$ \\
\hline LC08 & 2.54 & 6.68 & 6.39 & 6.12 & 6.40 & 0.28 & & & & & \\
\hline & $\begin{array}{rr}\text { Avg } & 2.7 \\
\text { SD } & 0.3 \\
\mathrm{~N} & \end{array}$ & & & & $\begin{array}{r}\text { Avg } \\
\text { SD } \\
\mathrm{N}\end{array}$ & $\begin{array}{r}4.57 \\
7.4 \\
2\end{array}$ & $\mathrm{~N}$ & & & & $\mathrm{~N}$ \\
\hline
\end{tabular}
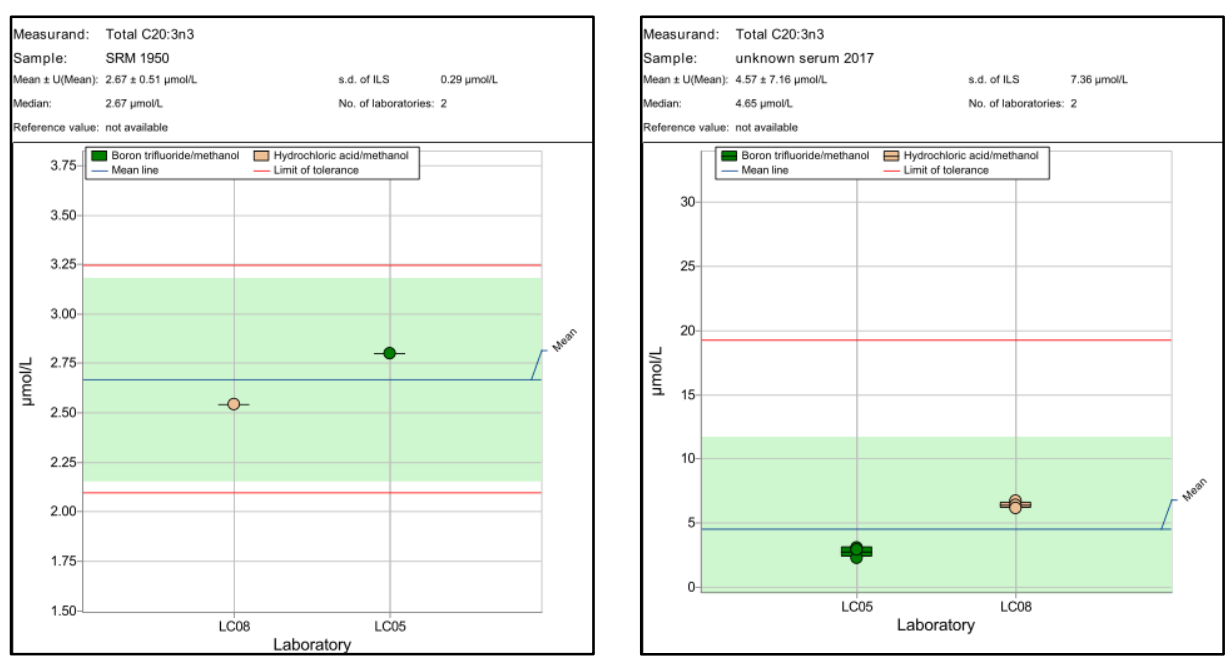
Table A-30: C20:3n6 homo- - -Linolenic acid, $\mu \mathrm{mol} / \mathrm{L}$

\begin{tabular}{|c|c|c|c|c|c|c|c|c|c|c|c|c|c|}
\hline & \multirow{2}{*}{$\frac{\text { FA }}{\text { SRM } 1950}$} & \multicolumn{5}{|c|}{2017 Unknown, FA } & \multicolumn{2}{|c|}{ FFA } & \multicolumn{5}{|c|}{2017 Unknown, FFA } \\
\hline Lab & & A & $\mathrm{B}$ & $\mathrm{C}$ & Avg & SD & SRM & 1950 & A & $\mathrm{B}$ & $\mathrm{C}$ & Avg & SD \\
\hline NIST & $139 \pm 4$ & & & & & & & & & & & & \\
\hline LC01 & 138 & 166 & 172 & 168 & 168 & 3.1 & & 2.27 & 3.80 & 3.34 & 4.04 & 3.73 & 0.36 \\
\hline LC02 & & 128 & 121 & 125 & 125 & 3.5 & & & & & & & \\
\hline LC04 & 106 & 123 & 132 & 128 & 128 & 4.6 & & & & & & & \\
\hline LC05 & 139 & 167 & 168 & 166 & 167 & 0.90 & & 0.848 & 1.87 & 1.81 & 1.76 & 1.81 & 0.056 \\
\hline LC07 & 162 & 216 & 200 & 206 & 207 & 8.1 & & & & & & & \\
\hline LC08 & 65.5 & 102 & 107 & 104 & 105 & 2 & & & & & & & \\
\hline LC09 & 60.6 & 65 & 65 & 61 & 63.9 & 2.38 & & 0.700 & 1.12 & 1.23 & 0.855 & 1.07 & 0.19 \\
\hline LC10 & 146 & 170 & 174 & 176 & 174 & 3.13 & & & & & & & \\
\hline LC11 & 183 & 174 & 173 & 176 & 174 & 1.44 & & & & & & & \\
\hline LC12 & 131 & 163 & 166 & 163 & 164 & 1.73 & & & & & & & \\
\hline LC14 & 127 & 162 & 162 & 148 & 157 & 8.1 & & & & & & & \\
\hline & Avg 127 & & & & Avg & & Avg & 1.27 & & & & Avg & 2.20 \\
\hline & SD $\quad 40$ & & & & SD & 30 & SD & 1.0 & & & & SD & 2.0 \\
\hline & 10 & & & & $\mathrm{~N}$ & 11 & $\mathrm{~N}$ & 3 & & & & $\mathrm{~N}$ & 3 \\
\hline
\end{tabular}
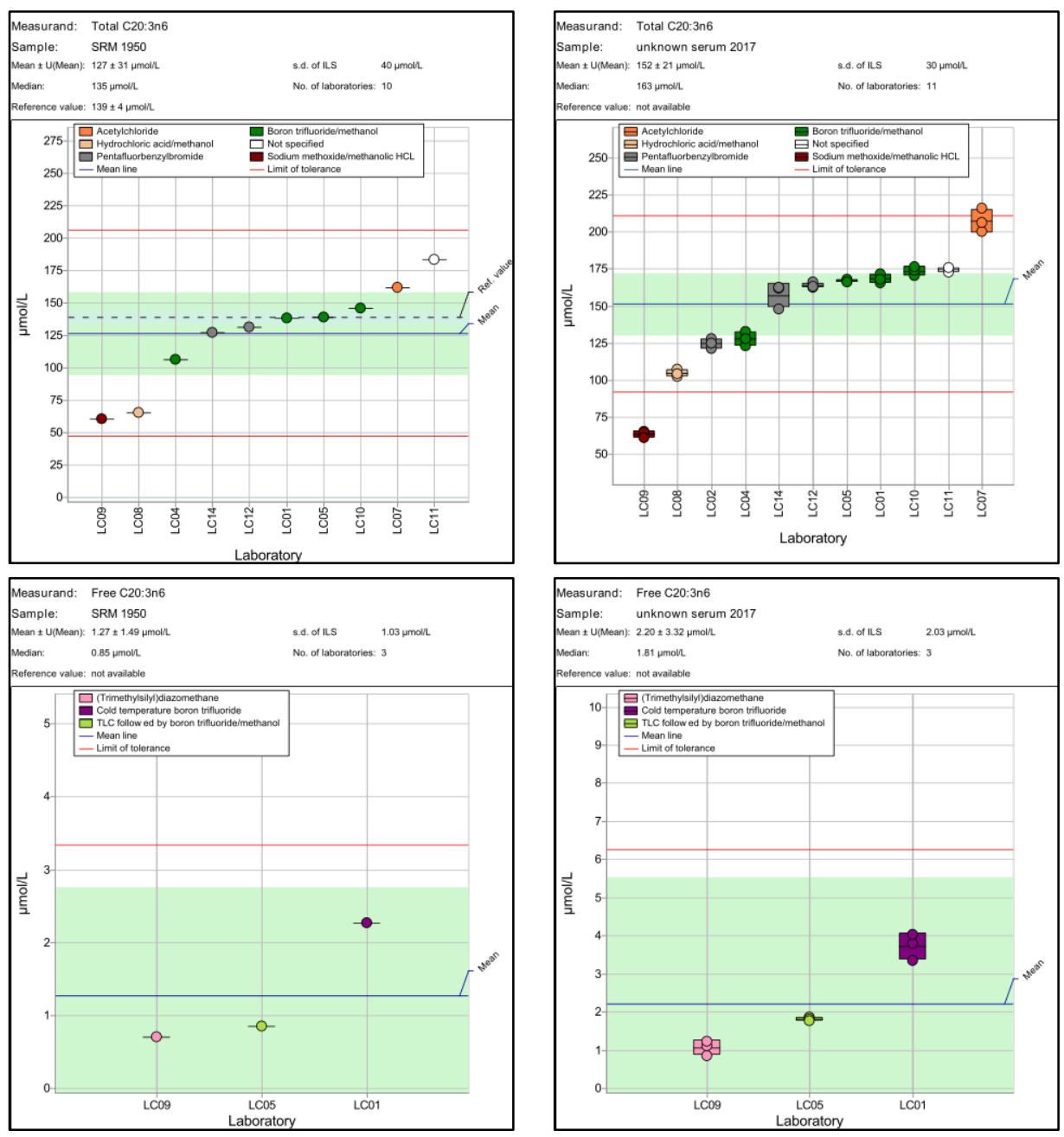
Table A-31: C20:3n9 5,8,11-Eicosatrienoic acid (Mead acid), $\mu \mathrm{mol} / \mathrm{L}$

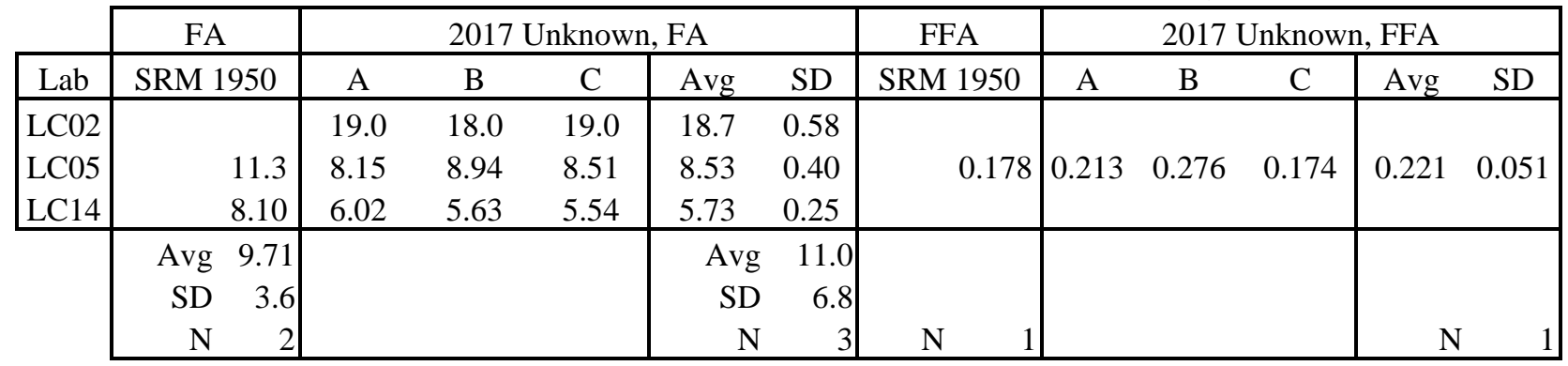
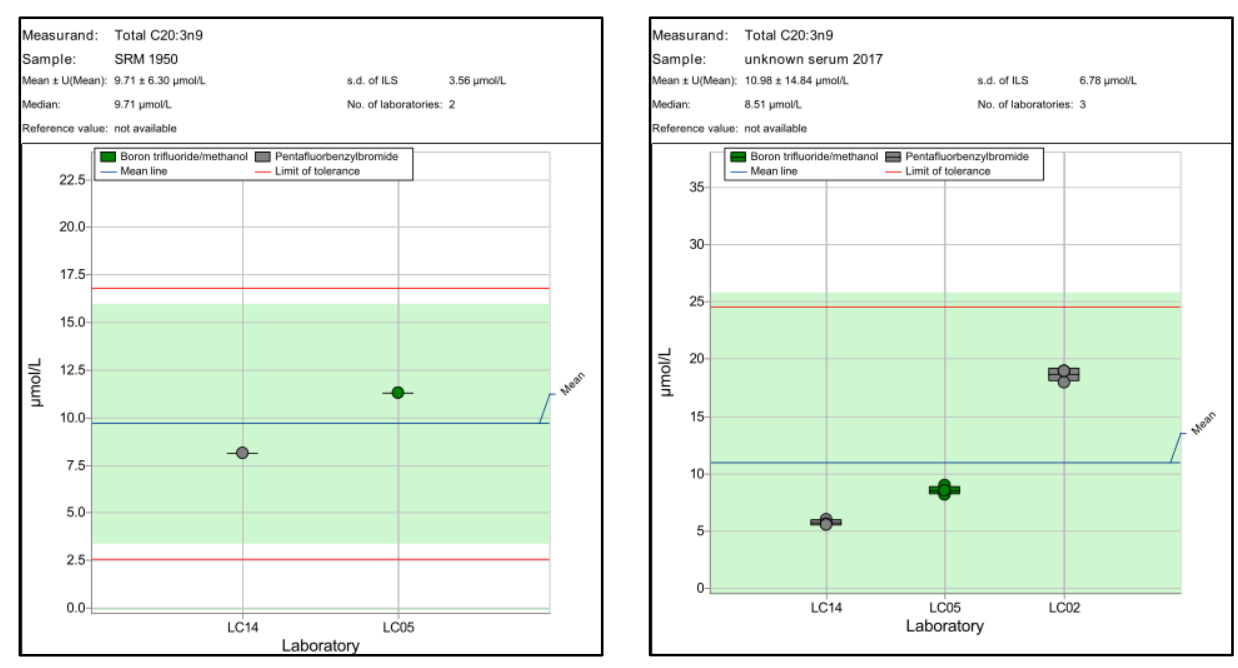
Table A-32: C20:4n6 Arachidonic acid, $\mu \mathrm{mol} / \mathrm{L}$

\begin{tabular}{|c|c|c|c|c|c|c|c|c|c|c|c|c|c|}
\hline & \multirow{2}{*}{$\frac{\text { FA }}{\text { SRM } 1950}$} & \multicolumn{5}{|c|}{2017 Unknown, FA } & \multicolumn{2}{|l|}{ FFA } & \multicolumn{5}{|c|}{2017 Unknown, FFA } \\
\hline Lab & & A & B & $\mathrm{C}$ & Avg & SD & SRM 19 & 950 & A & B & $\mathrm{C}$ & Avg & SD \\
\hline NIST & $984 \pm 180$ & & & & & & & & & & & & \\
\hline LC01 & 720 & 748 & 792 & 789 & 776 & 25 & & 1.4 & 19.9 & 18.1 & 19.2 & 19.1 & 0.87 \\
\hline LC02 & & 731 & 709 & 721 & 720 & 11 & & & & & & & \\
\hline LC04 & 495 & 529 & 587 & 561 & 559 & 29 & & & & & & & \\
\hline LC05 & 701 & 776 & 774 & 772 & 774 & 2.2 & & 3.83 & 10.9 & 10.8 & 10.7 & 10.8 & 0.097 \\
\hline LC07 & 510 & 616 & 570 & 594 & 593 & 23 & & & & & & & \\
\hline LC08 & 358 & 430 & 431 & 413 & 425 & 10 & & & & & & & \\
\hline LC09 & 489 & 458 & 445 & 428 & 443 & 15 & & .23 & 8.78 & 8.91 & 7.98 & 8.56 & 0.50 \\
\hline LC10 & 734 & 789 & 805 & 814 & 802 & 12 & & & & & & & \\
\hline LC11 & 853 & 715 & 720 & 722 & 719 & 3.5 & & & & & & & \\
\hline LC12 & 661 & 751 & 764 & 750 & 755 & 7.7 & & & & & & & \\
\hline LC14 & 627 & 800 & 801 & 683 & 761 & 68 & & & & & & & \\
\hline & Avg 615 & & & & Avg & & Avg & 7.16 & & & & Avg & 12.8 \\
\hline & SD & & & & SD & 110 & SD & 6.9 & & & & SD & 6.0 \\
\hline & 10 & & & & $\mathrm{~N}$ & 11 & $\mathrm{~N}$ & 3 & & & & $\mathrm{~N}$ & 3 \\
\hline
\end{tabular}
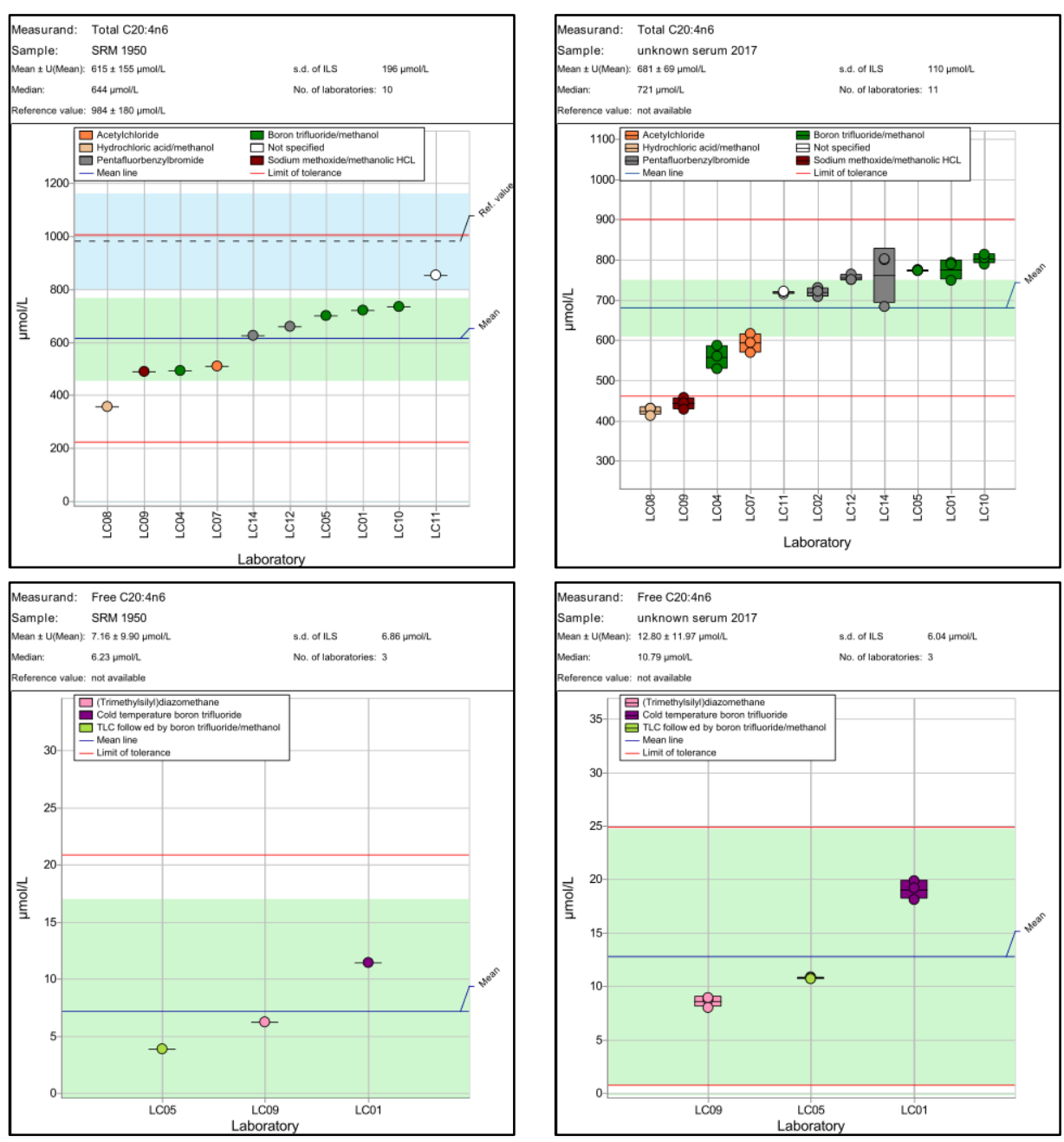
Table A-33: C20:5n3 Eicosapentaenoic acid, $\mu \mathrm{mol} / \mathrm{L}$

\begin{tabular}{|c|c|c|c|c|c|c|c|c|c|c|c|c|}
\hline & \multirow{2}{*}{$\frac{\text { FA }}{\text { SRM } 1950}$} & \multicolumn{5}{|c|}{2017 Unknown, FA } & FFA & \multicolumn{5}{|c|}{2017 Unknown, FFA } \\
\hline Lab & & A & $\mathrm{B}$ & $\mathrm{C}$ & Avg & SD & SRM 1950 & A & $\mathrm{B}$ & $\mathrm{C}$ & Avg & SD \\
\hline NIST & $38.6 \pm 0.5$ & & & & & & & & & & & \\
\hline LC01 & 35.3 & 40.5 & 47.3 & 53.8 & 47.2 & 6.7 & 0.257 & 2.34 & 0.465 & 1.01 & 1.27 & 0.96 \\
\hline LC02 & & 59.0 & 57.0 & 58.0 & 58.0 & 1.0 & & & & & & \\
\hline LC04 & 25.8 & 33.1 & 34.9 & 32.5 & 33.5 & 1.2 & & & & & & \\
\hline LC05 & 40.6 & 48.5 & 48.2 & 47.6 & 48.1 & 0.46 & 0.708 & 0.985 & 1.05 & 1.06 & 1.03 & 0.040 \\
\hline LC07 & 50.6 & 63.5 & 58.2 & 62.8 & 61.5 & 2.9 & & & & & & \\
\hline LC08 & 12.4 & 28.7 & 27.9 & 26.5 & 27.7 & 1.1 & & & & & & \\
\hline LC09 & 15.6 & 18.7 & 17.5 & 15.7 & 17.3 & 1.5 & & 0.470 & 0.534 & 0.343 & 0.449 & 0.10 \\
\hline LC10 & 40.6 & 45.9 & 47.0 & 47.4 & 46.8 & 0.80 & & & & & & \\
\hline LC11 & 58.0 & 57.1 & 58.1 & 60.2 & 58.4 & 1.6 & & & & & & \\
\hline LC12 & 33.4 & 41.4 & 41.8 & 42.1 & 41.8 & 0.37 & & & & & & \\
\hline & Avg 34.7 & & & & $\mathrm{Av} \xi$ & & Avg 0.482 & & & & Avg & 0.917 \\
\hline & SD 19 & & & & SI & 19 & SD $\quad 0.50$ & & & & SD & 1.0 \\
\hline & $\begin{array}{ll}\mathrm{N} & 9 \\
\end{array}$ & & & & $\mathrm{I}$ & 10 & $\mathrm{~N}$ & & & & $\mathrm{~N}$ & 3 \\
\hline
\end{tabular}
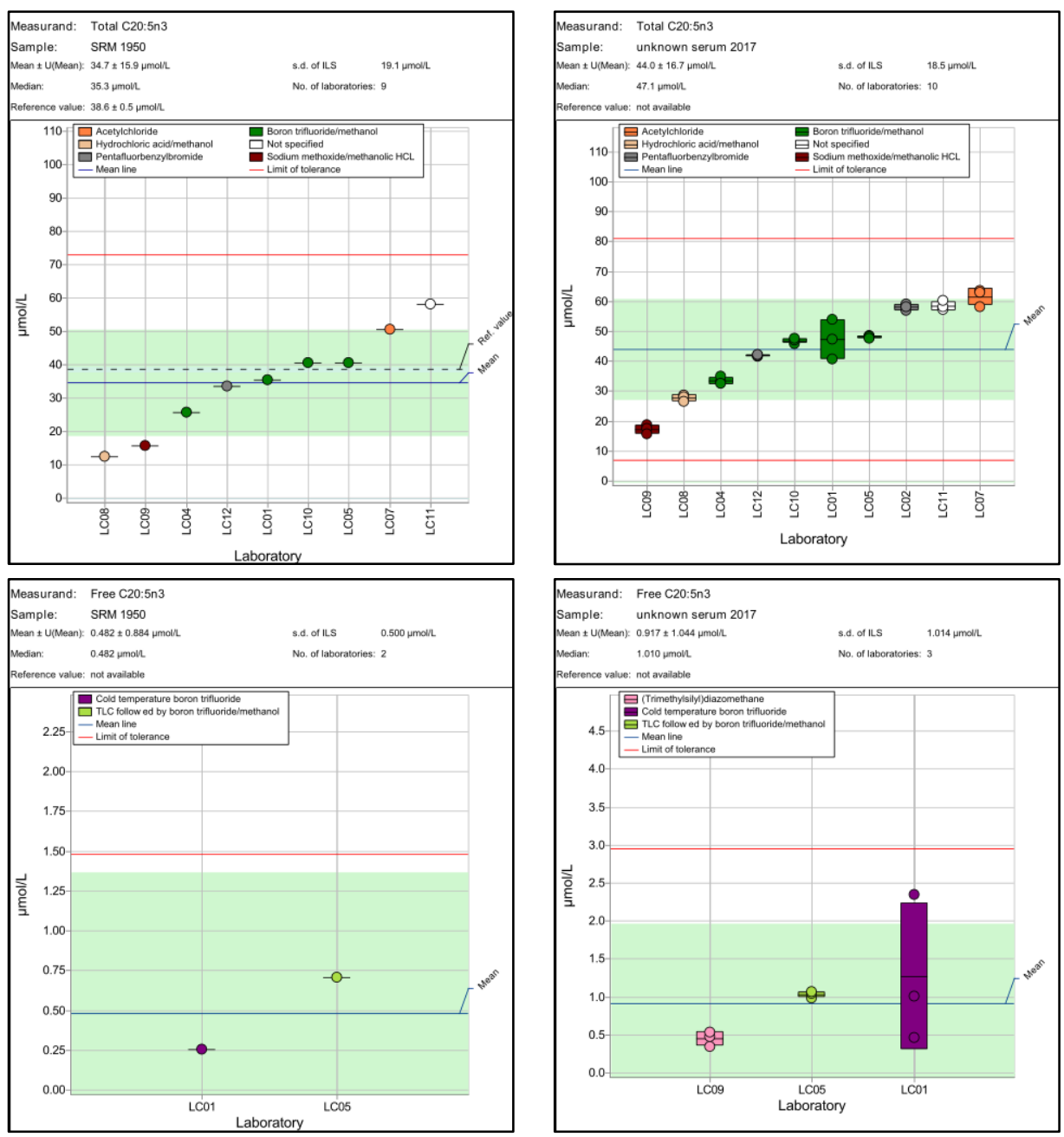
Table A-34: C22:0 Docosanoic acid, $\mu \mathrm{mol} / \mathrm{L}$

\begin{tabular}{|c|c|c|c|c|c|c|c|c|c|c|c|c|}
\hline & FA & \multicolumn{5}{|c|}{2017 Unknown, FA } & FFA & \multicolumn{5}{|c|}{2017 Unknown, FFA } \\
\hline Lab & SRM 1950 & A & B & $\mathrm{C}$ & Avg & SD & SRM 1950 & A & B & $\mathrm{C}$ & Avg & SD \\
\hline NIST & $47.8 \pm 4.6$ & & & & & & & & & & & \\
\hline LC01 & 19.4 & 27.2 & 29.6 & 27.9 & 28.2 & 1.3 & 0.435 & 0.684 & 0.317 & 0.293 & 0.431 & 0.22 \\
\hline LC02 & 54.0 & 79.3 & 75.7 & 77.7 & 77.6 & 1.8 & & & & & & \\
\hline LC04 & 44.3 & 67.0 & 74.2 & 76.3 & 72.5 & 4.9 & & & & & & \\
\hline LC05 & 43.8 & 62.0 & 62.2 & 64.1 & 62.8 & 1.2 & 0.618 & 0.567 & 0.531 & 0.571 & 0.556 & 0.022 \\
\hline LC07 & 52.3 & 83.1 & 77.0 & 82.5 & 80.9 & 3.4 & & & & & & \\
\hline LC08 & 31.5 & 41.2 & 37.8 & 38.1 & 39.0 & 1.9 & & & & & & \\
\hline LC11 & 50.1 & 52.0 & 47.5 & 52.0 & 50.5 & 2.6 & & & & & & \\
\hline LC12 & 50.4 & 73.9 & 75.6 & 75.4 & 75.0 & 0.90 & & & & & & \\
\hline & Avg 44.3 & & & & $\mathrm{Av}$ & 60.8 & Avg 0.526 & & & & Avg & 0.494 \\
\hline & SD 11 & & & & SI & 22 & SD $\quad 0.20$ & & & & SD & 0.32 \\
\hline & $\mathrm{N}$ & & & & $\mathrm{I}$ & 8 & $\mathrm{~N}$ & & & & $\mathrm{~N}$ & 2 \\
\hline
\end{tabular}
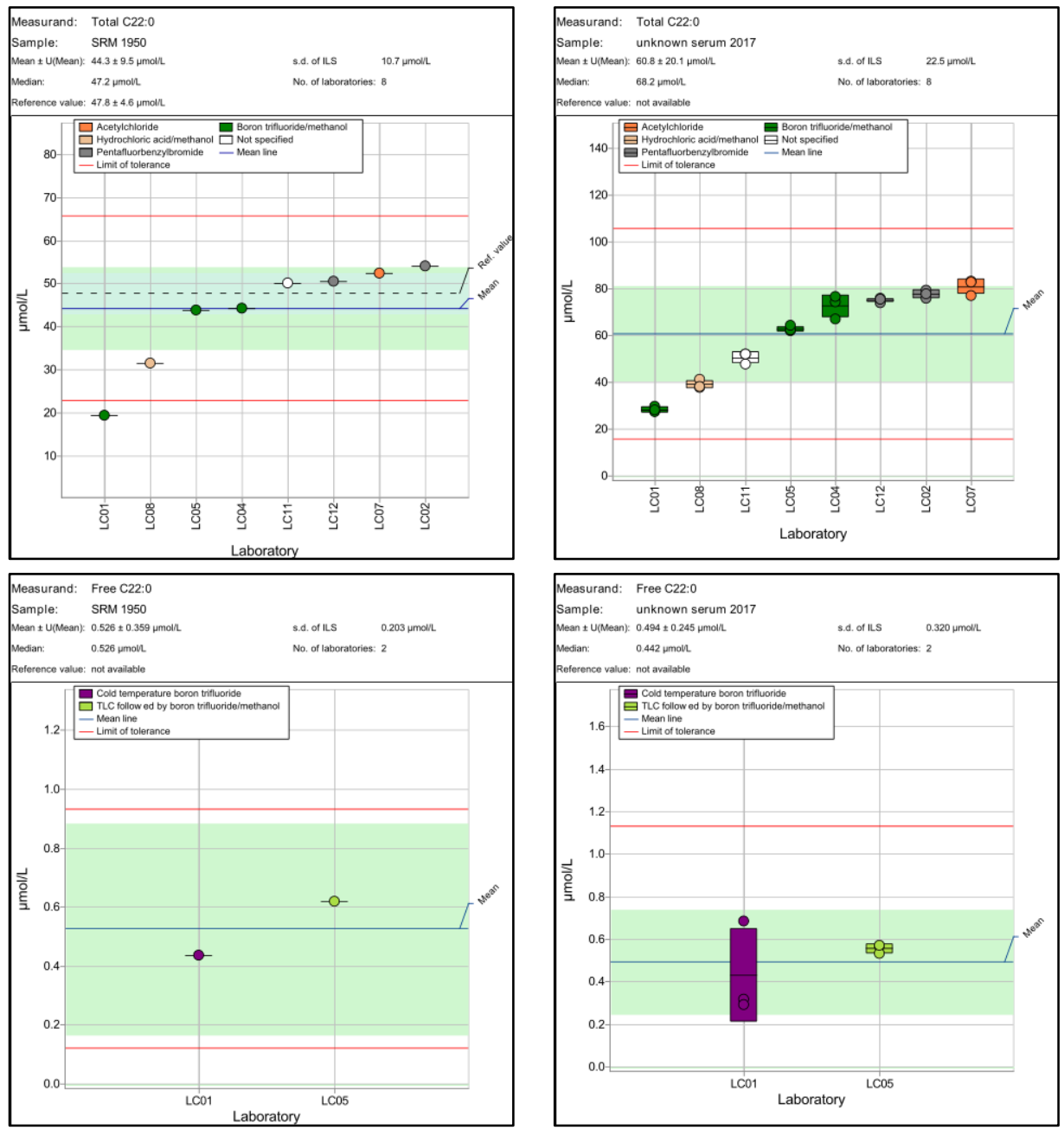
Table A-35: C22:1n9 Docosenoic acid, $\mu \mathrm{mol} / \mathrm{L}$

\begin{tabular}{|c|c|c|c|c|c|c|c|c|c|c|c|c|}
\hline & FA & & 2017 & nknow & FA & & FFA & & 2017 & nkno & FFA & \\
\hline Lab & SRM 1950 & A & B & $\mathrm{C}$ & Avg & SD & SRM 1950 & A & B & $\mathrm{C}$ & Avg & SD \\
\hline LC02 & & 10.0 & 10.0 & 10.0 & 10.0 & 0.0 & \multirow{6}{*}{0.984} & \multirow{6}{*}{1.32} & \multirow{6}{*}{1.20} & \multirow{6}{*}{1.35} & \multirow{6}{*}{1.29} & \multirow{6}{*}{0.078} \\
\hline LC04 & 3.58 & 4.37 & 3.25 & 2.53 & 3.38 & 0.92 & & & & & & \\
\hline LC05 & 3.43 & 3.95 & 3.51 & 4.60 & 4.02 & 0.55 & & & & & & \\
\hline LC08 & 10.9 & 0.960 & 0.580 & 0.360 & 0.633 & 0.30 & & & & & & \\
\hline LC10 & 27.1 & 12.4 & $<$ LOD & 10.2 & 11.3 & 1.6 & & & & & & \\
\hline \multirow[t]{4}{*}{ LC11 } & 14.1 & 16.9 & 14.5 & 15.5 & 15.6 & 1.2 & & & & & & \\
\hline & Avg 11.8 & & & & Avg & 7.50 & & & & & & \\
\hline & $\mathrm{SD} \quad 16$ & & & & SD & 8.0 & & & & & & \\
\hline & $\mathrm{N}$ & & & & $\mathrm{N}$ & 6 & $\mathrm{~N}$ & & & & & 1 \\
\hline
\end{tabular}
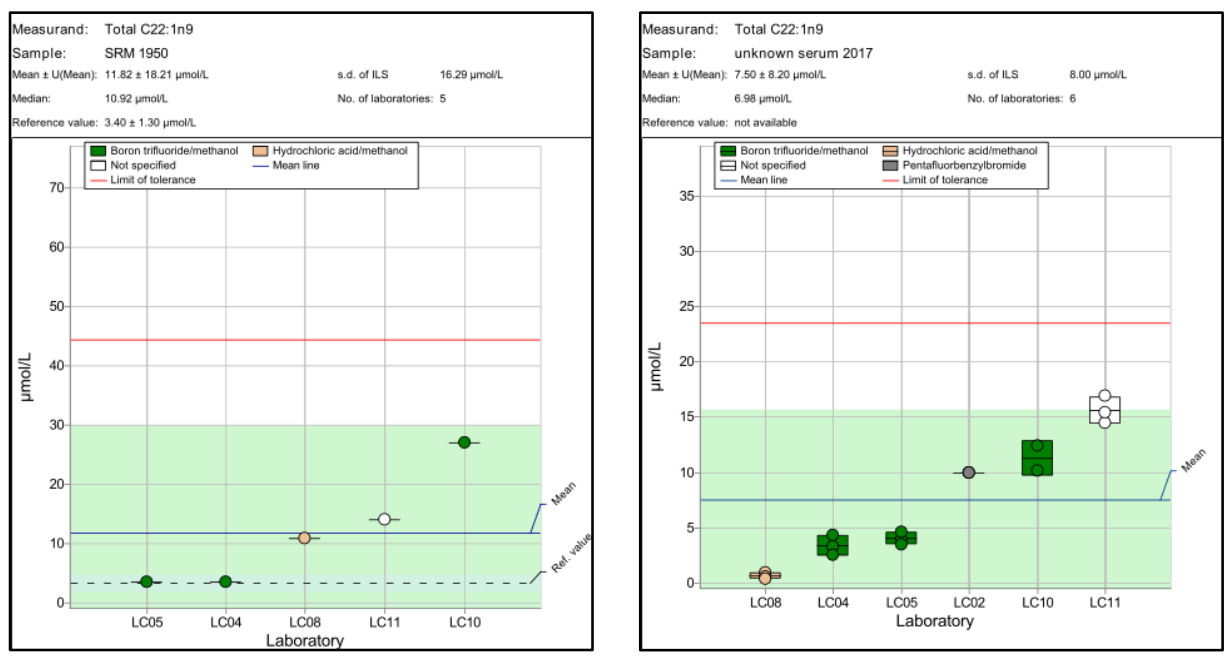
Table A-36: C22:2n6 Docosadienoic acid, $\mu \mathrm{mol} / \mathrm{L}$

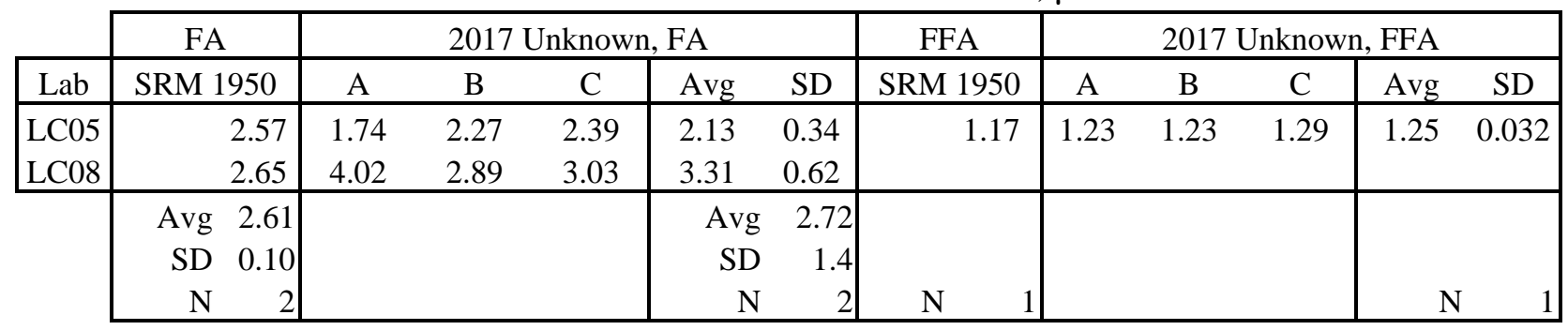
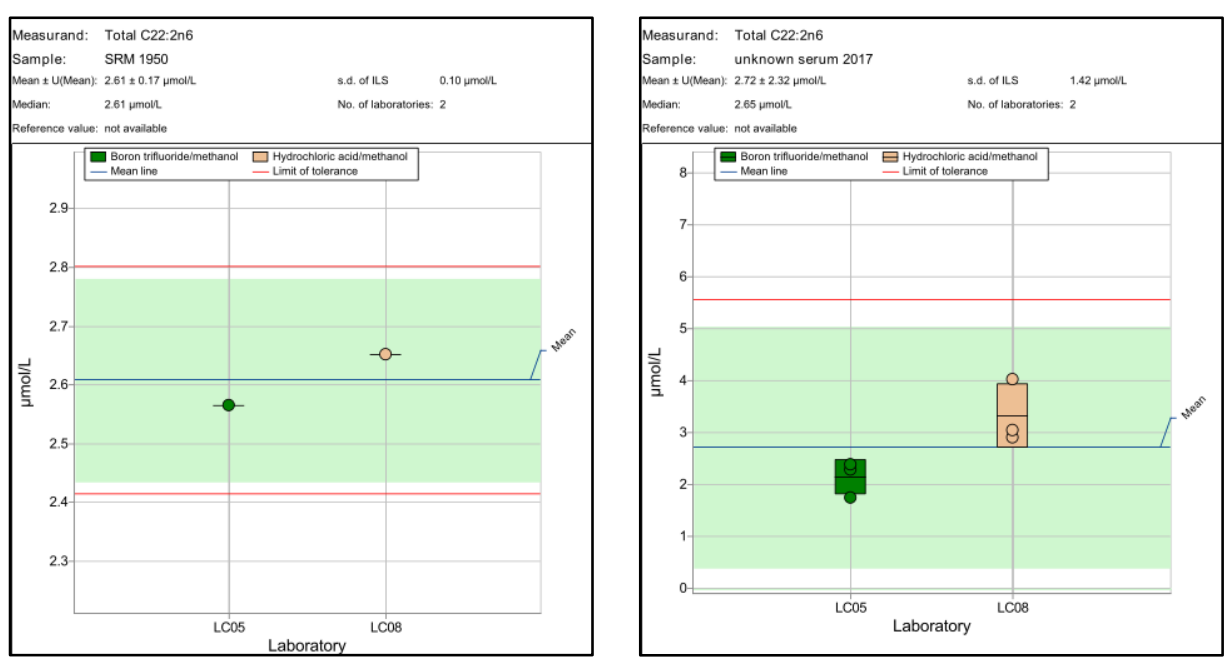
Table A-37: C22:4n6 Docosatetraenoic acid, $\mu \mathrm{mol} / \mathrm{L}$

\begin{tabular}{|c|c|c|c|c|c|c|c|c|c|c|c|c|}
\hline & \multirow{2}{*}{$\frac{\text { FA }}{\text { SRM } 1950}$} & \multicolumn{5}{|c|}{2017 Unknown, FA } & FFA & \multicolumn{5}{|c|}{2017 Unknown, FFA } \\
\hline Lab & & A & B & $\mathrm{C}$ & Avg & SD & SRM 1950 & A & B & $\mathrm{C}$ & Avg & SD \\
\hline NIST & $25.5 \pm 0.6$ & & & & & & & & & & & \\
\hline LC01 & 30.8 & 24.8 & 25.9 & 29.4 & 26.7 & 2.4 & 0.992 & 0.416 & 0.587 & 0.739 & 0.581 & 0.16 \\
\hline LC02 & & 27.0 & 27.0 & 27.0 & 27.0 & 0.0 & & & & & & \\
\hline LC04 & 18.1 & 19.4 & 17.7 & 18.5 & 18.5 & 0.85 & & & & & & \\
\hline LC05 & 23.3 & 23.9 & 24.3 & 24.0 & 24.0 & 0.18 & 0.67 & 0.740 & 0.770 & 0.677 & 0.729 & 0.047 \\
\hline LC09 & 18.9 & 20.4 & 13.0 & 16.0 & 16.5 & 3.7 & & 0.000 & 0.0900 & 0.0490 & 0.0463 & 0.045 \\
\hline LC10 & 28.5 & 25.6 & 25.9 & 28.7 & 26.7 & 1.7 & & & & & & \\
\hline LC11 & 23.1 & 19.9 & 20.5 & 21.0 & 20.5 & 0.54 & & & & & & \\
\hline LC12 & 23.0 & 24.1 & 23.9 & 23.8 & 23.9 & 0.13 & & & & & & \\
\hline LC14 & 26.0 & 23.9 & 25.0 & 24.1 & 24.4 & 0.59 & & & & & & \\
\hline & Avg 24.0 & & & & $\mathrm{Av}$ & 23.2 & Avg 0.83 & & & & Avg & 0.452 \\
\hline & SD $\quad 6.1$ & & & & SI & 4.3 & SD $\quad 0.3$ & & & & SD & 0.61 \\
\hline & 8 & & & & I & 9 & $\mathrm{~N}$ & 2 & & & $\mathrm{~N}$ & 3 \\
\hline
\end{tabular}
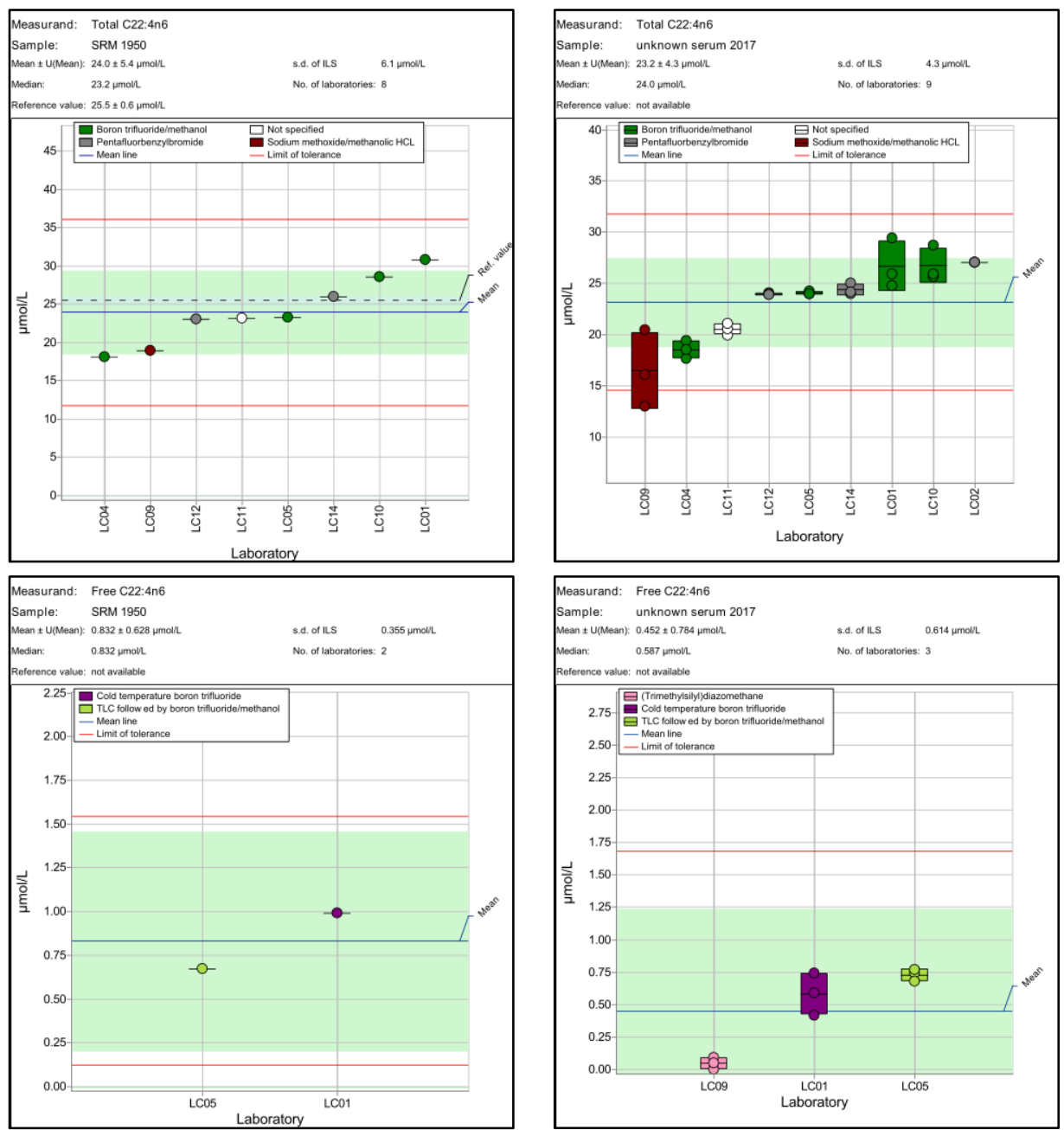
Table A-38: C22:5n3 Docosapentaenoic acid, $\mu \mathrm{mol} / \mathrm{L}$

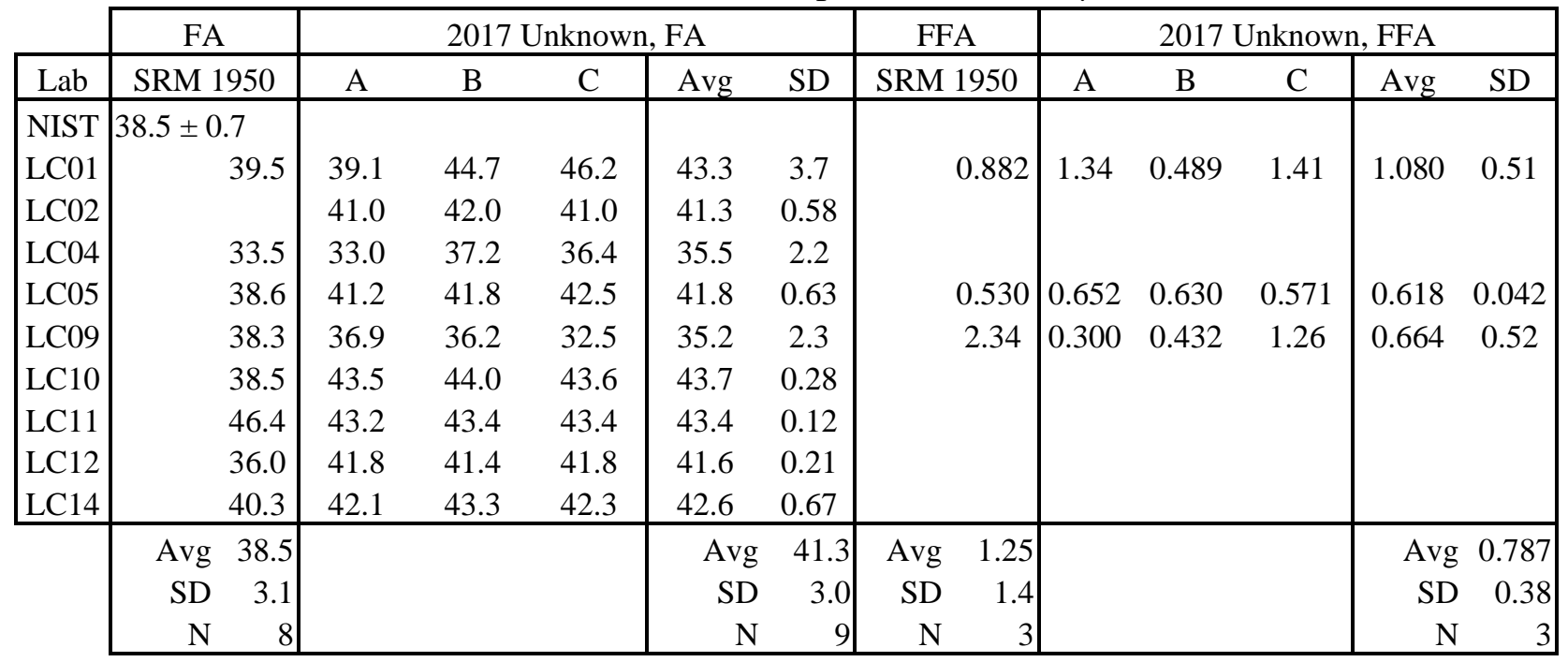
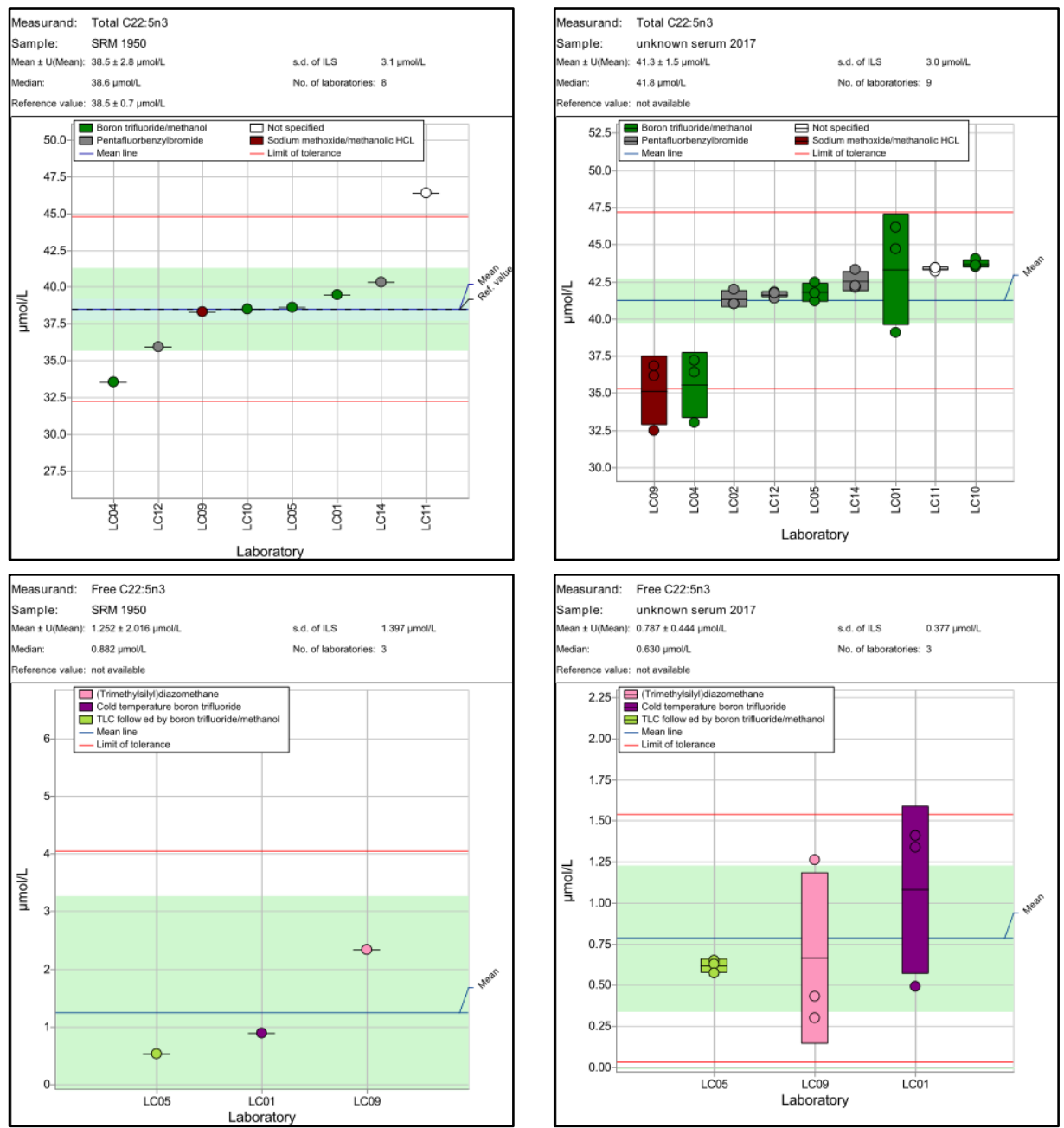
Table A-39: C22:5n6 Docosapentaenoic acid, $\mu \mathrm{mol} / \mathrm{L}$

\begin{tabular}{|c|c|c|c|c|c|c|c|c|c|c|c|c|}
\hline & \multirow{2}{*}{$\frac{\text { FA }}{\text { SRM } 1950}$} & \multicolumn{5}{|c|}{2017 Unknown, FA } & FFA & \multicolumn{5}{|c|}{2017 Unknown, FFA } \\
\hline Lab & & A & B & $\mathrm{C}$ & Avg & SD & SRM 1950 & A & B & $\mathrm{C}$ & Avg & SD \\
\hline NIST & $19.5 \pm 0.4$ & & & & & & & & & & & \\
\hline LC01 & 19.4 & 35.0 & 28.1 & 31.0 & 31.4 & 3.5 & 0.509 & 0.324 & 0.282 & 0.741 & 0.449 & 0.25 \\
\hline LC02 & & 35.0 & 34.0 & 35.0 & 34.7 & 0.58 & & & & & & \\
\hline LC04 & 14.2 & 16.7 & 19.7 & 18.9 & 18.5 & 1.6 & & & & & & \\
\hline LC05 & 17.4 & 27.0 & 26.7 & 27.1 & 26.9 & 0.19 & 0.583 & 0.620 & 0.623 & 1.017 & 0.753 & 0.228 \\
\hline LC09 & 17.4 & 22.2 & 16.1 & 16.4 & 18.2 & 3.4 & & 0.755 & 0.442 & 0.0700 & 0.422 & 0.34 \\
\hline LC10 & 21.0 & 28.4 & 28.8 & 29.9 & 29.0 & 0.80 & & & & & & \\
\hline LC11 & 27.3 & 71.6 & 65.2 & 59.2 & 65.3 & 6.2 & & & & & & \\
\hline LC12 & 17.0 & 26.4 & 26.8 & 26.4 & 26.6 & 0.24 & & & & & & \\
\hline LC14 & 19.4 & 27.4 & 28.0 & 27.0 & 27.5 & 0.48 & & & & & & \\
\hline & Avg 18.9 & & & & $\mathrm{Av}$ & 26.6 & Avg 0.546 & & & & Avg & 0.542 \\
\hline & SD 4.3 & & & & SI & 7.9 & SD 0.082 & & & & $\mathrm{SD}$ & 0.41 \\
\hline & $\mathrm{N}$ & & & & & 9 & $\mathrm{~N}$ & & & & $\mathrm{~N}$ & 3 \\
\hline
\end{tabular}
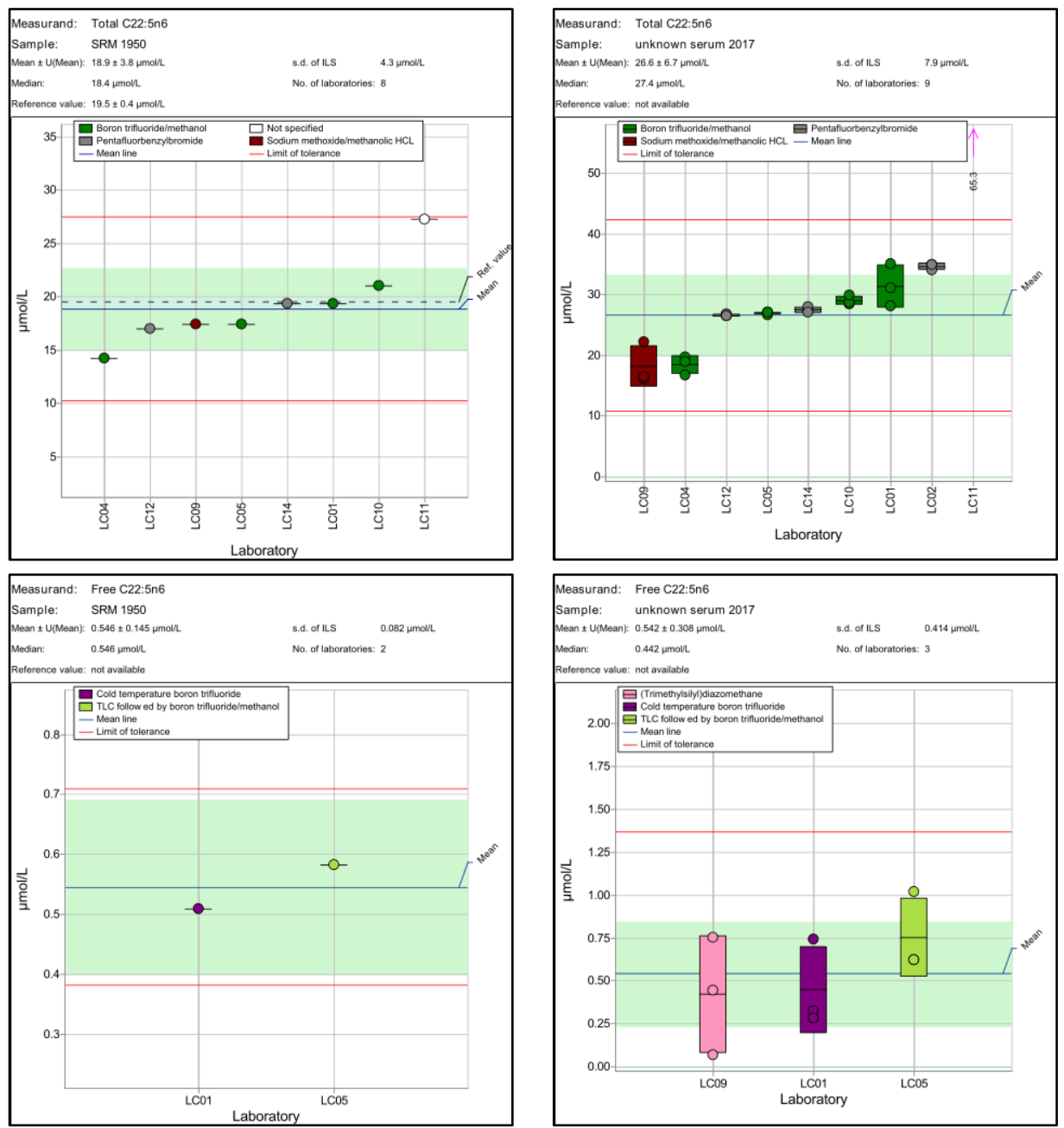
Table A-40: C22:6n3 Docosahexaenoic acid, $\mu \mathrm{mol} / \mathrm{L}$

\begin{tabular}{|c|c|c|c|c|c|c|c|c|c|c|c|c|}
\hline \multirow[b]{2}{*}{ Lab } & FA & \multicolumn{5}{|c|}{2017 Unknown, FA } & FFA & \multicolumn{5}{|c|}{2017 Unknown, FFA } \\
\hline & SRM 1950 & A & B & $\mathrm{C}$ & Avg & SD & SRM 1950 & A & B & $\mathrm{C}$ & Avg & SD \\
\hline NIST & $118 \pm 21$ & & & & & & & & & & & \\
\hline LC01 & 104 & 175 & 183 & 185 & 181 & 5.5 & 2.18 & 3.16 & 4.40 & 4.02 & 3.86 & 0.64 \\
\hline LC02 & & 181 & 177 & 179 & 179 & 2.0 & & & & & & \\
\hline LC04 & 78.0 & 127 & 139 & 135 & 134 & 6.2 & & & & & & \\
\hline LC05 & 103 & 170 & 172 & 174 & 172 & 1.8 & 1.23 & 2.74 & 2.82 & 2.77 & 2.78 & 0.042 \\
\hline LC07 & 120 & 244 & 215 & 225 & 228 & 15 & & & & & & \\
\hline LC08 & 165 & 95.2 & 97.5 & 95.3 & 96.0 & 1.3 & & & & & & \\
\hline LC09 & 103 & 150 & 140 & 131 & 140 & 9.6 & 3.64 & 4.07 & 4.57 & 5.24 & 4.62 & 0.59 \\
\hline LC10 & 105 & 176 & 180 & 181 & 179 & 2.6 & & & & & & \\
\hline LC11 & 126 & 176 & 170 & 170 & 172 & 3.0 & & & & & & \\
\hline LC12 & 91.7 & 169 & 169 & 169 & 169 & 0.29 & & & & & & \\
\hline & Avg 109 & & & & Avg & & Avg 2.35 & & & & Avg & 3.75 \\
\hline & SD 26 & & & & SD & 20 & SD & & & & SD & 1.37 \\
\hline & $\mathrm{N}$ & & & & $\mathrm{N}$ & 10 & $\mathrm{~N}$ & & & & $\mathrm{~N}$ & 3 \\
\hline
\end{tabular}
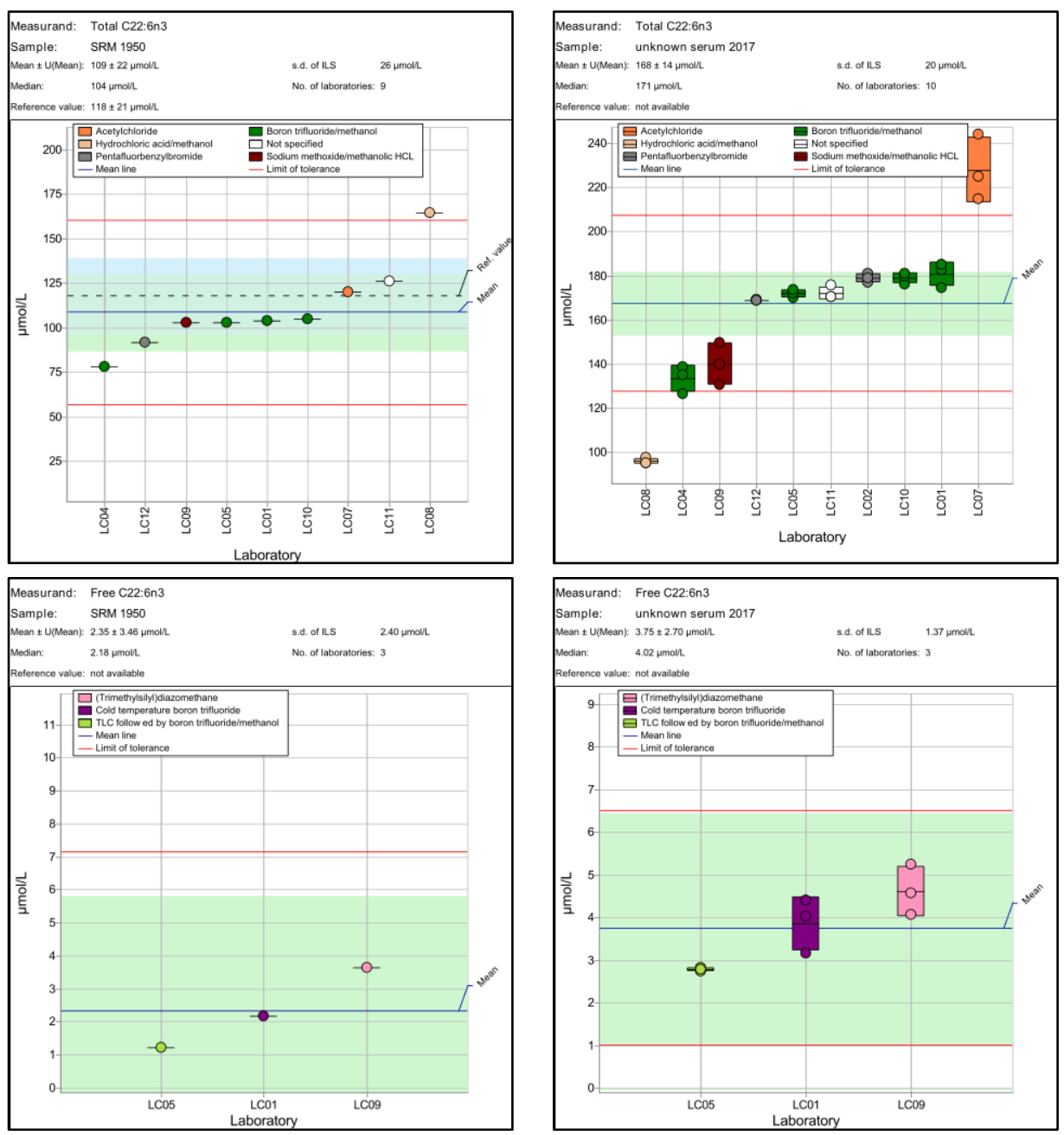
Table A-41: C23:0 Tricosanoic acid, $\mu \mathrm{mol} / \mathrm{L}$

\begin{tabular}{|c|c|c|c|c|c|c|c|c|c|c|c|}
\hline & FA & \multicolumn{5}{|c|}{2017 Unknown, FA } & FFA & \multicolumn{4}{|c|}{2017 Unknown, FFA } \\
\hline Lab & SRM 1950 & A & B & $\mathrm{C}$ & Avg & SD & SRM 1950 & A & $\mathrm{B}$ & $\mathrm{C}$ & Avg \\
\hline LC05 & 15.0 & 24.2 & 24.6 & 24.8 & 24.5 & 0.28 & 0.142 & 0.229 & 0.213 & 0.174 & $0.205 \quad 0.028$ \\
\hline LC08 & 3.52 & 14.0 & 13.5 & 13.2 & 13.6 & 0.39 & & & & & \\
\hline LC11 & 12.1 & 13.5 & 10.1 & 12.9 & 12.2 & 1.8 & & & & & \\
\hline LC14 & 19.7 & 31.2 & 31.0 & 30.4 & 30.9 & 0.41 & & & & & \\
\hline & Avg 12.6 & & & & Avg & 20.3 & & & & & \\
\hline & SD 10.4 & & & & SD & 14.3 & & & & & \\
\hline & $\mathrm{N}$ & & & & $\mathrm{N}$ & 4 & $\mathrm{~N}$ & & & & $\mathrm{~N}$ \\
\hline
\end{tabular}
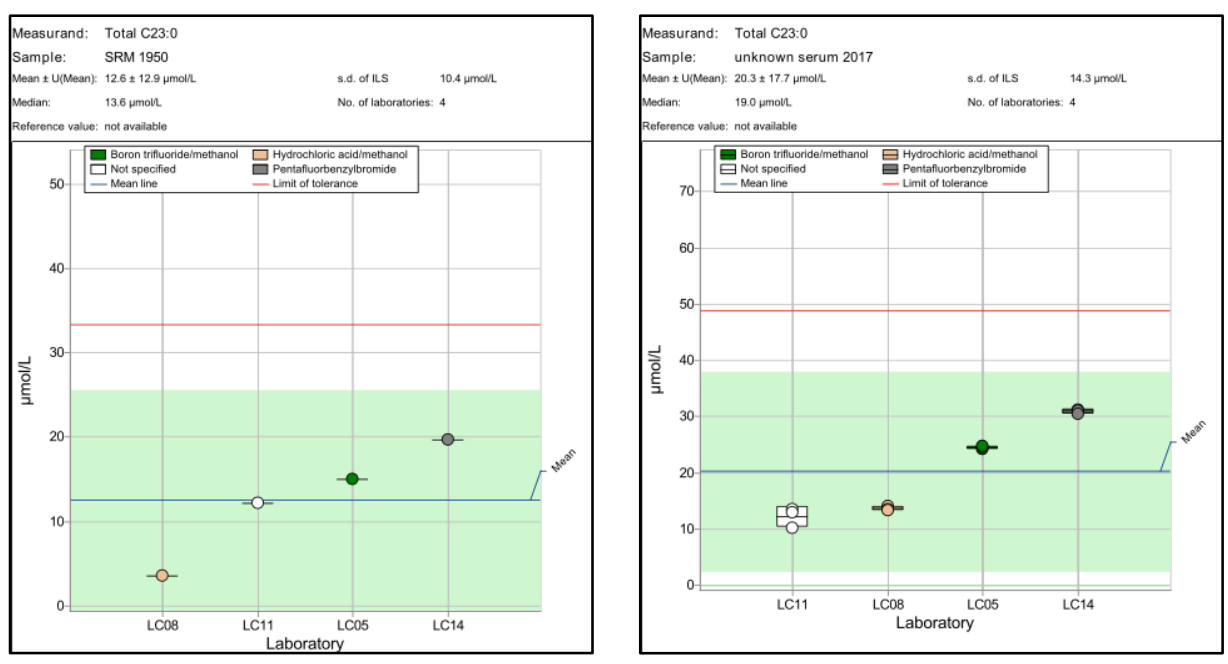
Table A-42: C24:0 Lignoceric acid, $\mu \mathrm{mol} / \mathrm{L}$

\begin{tabular}{|c|c|c|c|c|c|c|c|c|c|c|c|c|}
\hline & FA & \multicolumn{5}{|c|}{2017 Unknown, FA } & FFA & \multicolumn{5}{|c|}{2017 Unknown, FFA } \\
\hline Lab & SRM 1950 & A & B & $\mathrm{C}$ & Avg & SD & SRM 1950 & A & B & C & Avg & SD \\
\hline NIST & $46.6 \pm 2.6$ & & & & & & & & & & & \\
\hline LC01 & 23.5 & 18.7 & 23.0 & 19.7 & 20.4 & 2.2 & 0.568 & 0.661 & 0.444 & 0.349 & 0.485 & 0.16 \\
\hline LC02 & & 60.4 & 57.3 & 58.9 & 58.9 & 1.6 & & & & & & \\
\hline LC04 & 28.6 & 40.9 & 42.7 & 42.0 & 41.9 & 0.90 & & & & & & \\
\hline LC05 & 37.9 & 47.2 & 48.6 & 50.8 & 48.8 & 1.8 & 0.507 & 0.467 & 0.529 & 0.601 & 0.532 & 0.067 \\
\hline LC08 & 34.0 & 27.5 & 26.5 & 27.3 & 27.1 & 0.52 & & & & & & \\
\hline LC11 & 48.4 & 58.9 & 51.9 & 53.4 & 54.7 & 3.7 & & & & & & \\
\hline LC12 & 41.9 & 56.1 & 56.8 & 57.0 & 56.7 & 0.48 & & & & & & \\
\hline LC14 & 41.1 & 57.3 & 56.7 & 55.7 & 56.6 & 0.83 & & & & & & \\
\hline & Avg 36.5 & & & & Avg & 46.7 & Avg 0.538 & & & & Avg & 0.509 \\
\hline & SD 12 & & & & $\mathrm{SD}$ & 13 & SD 0.068 & & & & SD & 0.18 \\
\hline & $\mathrm{N}$ & & & & $\mathrm{N}$ & 8 & $\mathrm{~N}$ & & & & $\mathrm{~N}$ & 2 \\
\hline
\end{tabular}
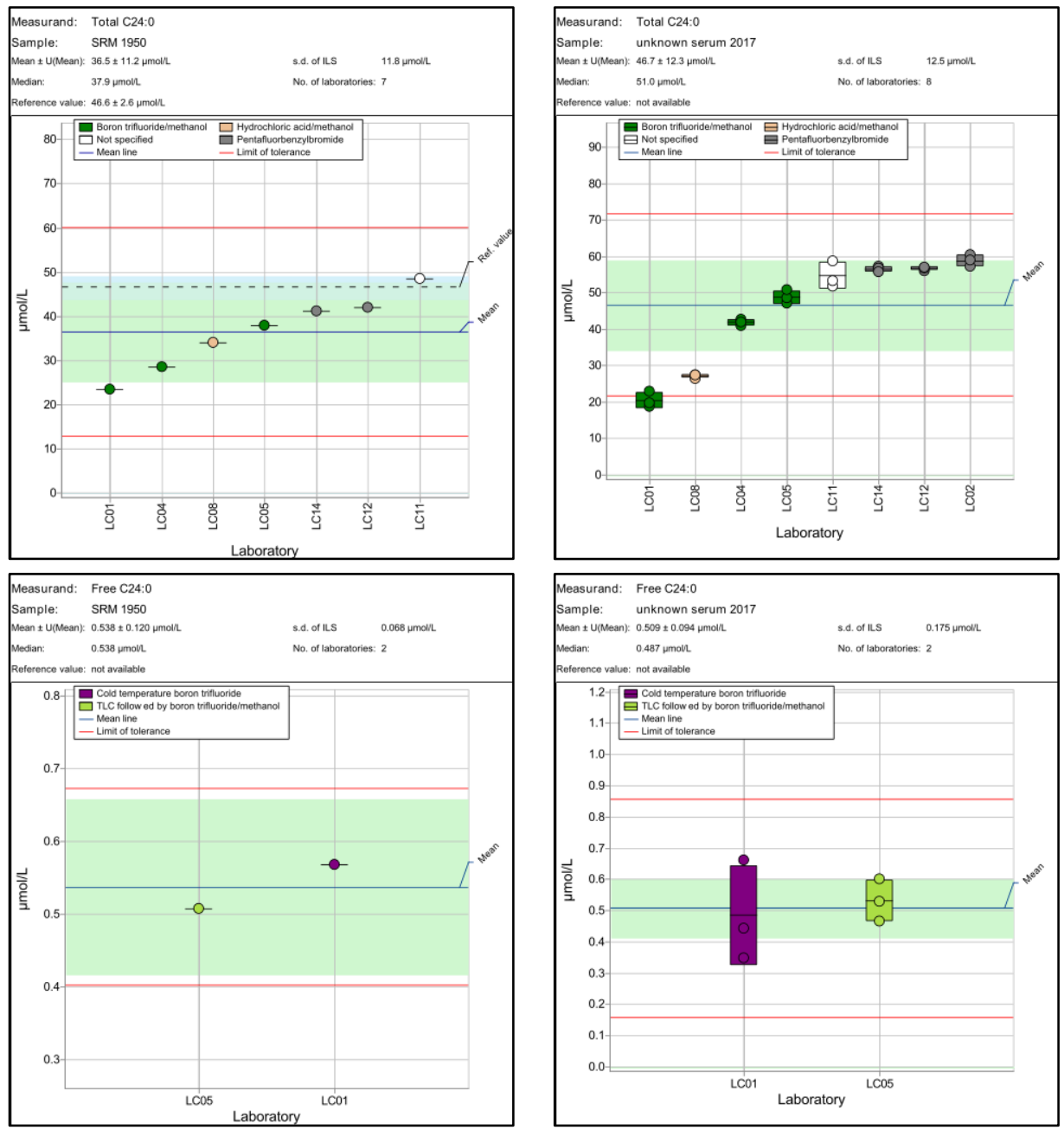
Table A-43: C24:1n9 Nervonic acid, $\mu \mathrm{mol} / \mathrm{L}$

\begin{tabular}{|c|c|c|c|c|c|c|c|c|c|c|c|c|}
\hline \multirow[b]{2}{*}{ Lab } & FA & \multicolumn{5}{|c|}{2017 Unknown, FA } & FFA & \multicolumn{5}{|c|}{2017 Unknown, FFA } \\
\hline & SRM 1950 & A & B & C & Avg & SD & SRM 1950 & A & B & C & Avg & SD \\
\hline NIST & $71.3 \pm 3.2$ & & & & & & & & & & & \\
\hline LC01 & 29.2 & 22.9 & 20.2 & 19.7 & 20.9 & 1.7 & 0.271 & 0.540 & 0.362 & 0.314 & 0.405 & 0.12 \\
\hline LC02 & & 77.0 & 72.0 & 74.0 & 74.3 & 2.5 & & & & & & \\
\hline LC04 & 49.0 & 68.5 & 69.1 & 76.3 & 71.3 & 4.4 & & & & & & \\
\hline LC05 & 52.2 & 57.6 & 58.4 & 59.5 & 58.5 & 1.0 & 0.132 & 0.139 & 0.138 & 0.088 & 0.122 & 0.029 \\
\hline LC07 & 68.7 & 81.0 & 76.4 & 79.6 & 79.0 & 2.4 & & & & & & \\
\hline LC11 & 79.7 & 51.9 & 65.8 & 76.1 & 64.6 & 12 & & & & & & \\
\hline LC12 & 63.1 & 69.9 & 71.7 & 72.0 & 71.2 & 1.1 & & & & & & \\
\hline LC14 & 67.4 & 77.5 & 74.3 & 74.2 & 75.3 & 1.8 & & & & & & \\
\hline & Avg 58.5 & & & & Avg & 70.6 & Avg 0.201 & & & & Avg & 0.263 \\
\hline & SD 24 & & & & SD & 10 & SD $\quad 0.15$ & & & & SD & 0.47 \\
\hline & $\mathrm{N}$ & & & & $\mathrm{N}$ & 8 & $\mathrm{~N}$ & & & & $\mathrm{~N}$ & 2 \\
\hline
\end{tabular}
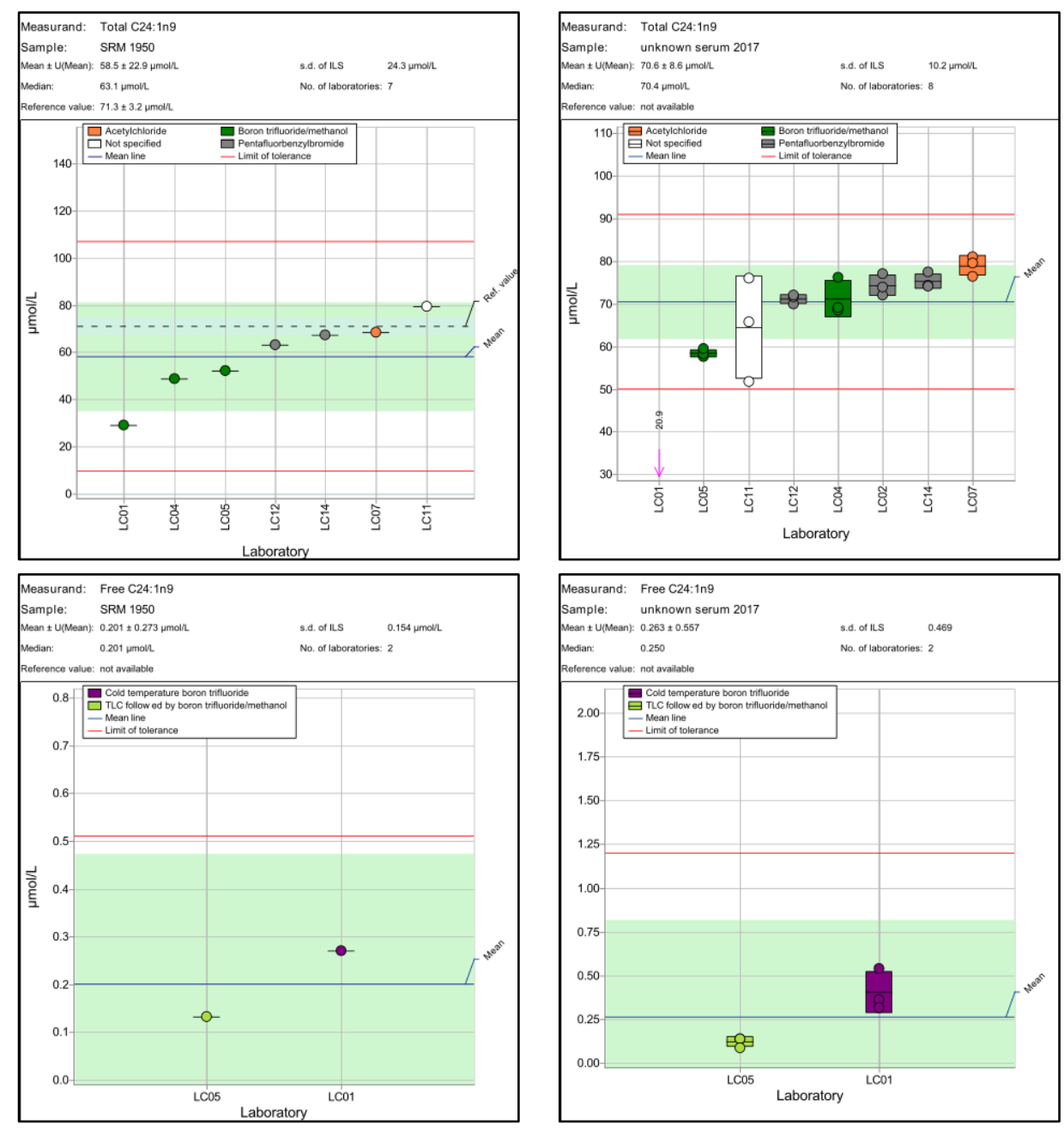


\section{Appendix B: Free Fatty Acids and Fatty Acid Methyl Esters in Solutions}

\section{List of Tables}

Table B-1: C16:1n5 cis-11-hexadecenoic acid, $\mu$ mol/L ................................................................ 59

Table B-2: C16:1n5t trans-11-Hexadecenoic acid, $\mu$ mol/L........................................................... 59

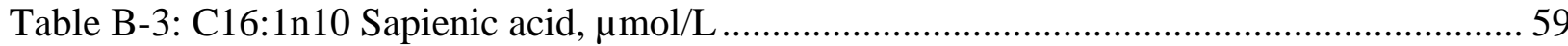

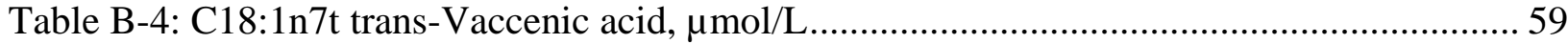

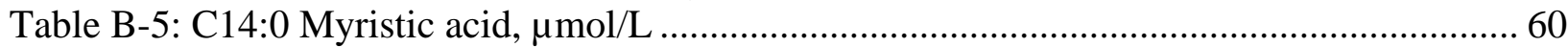

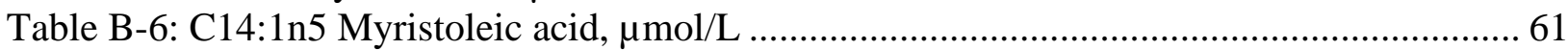

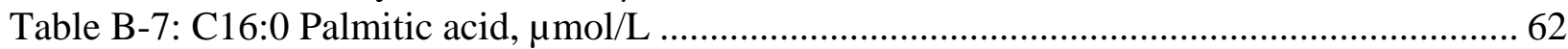

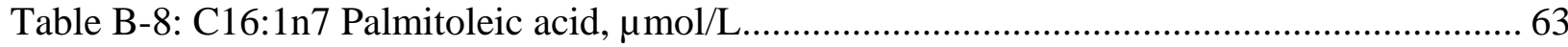

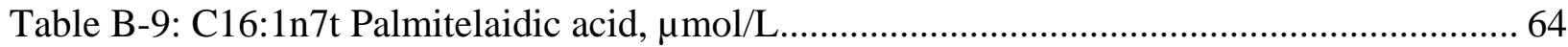

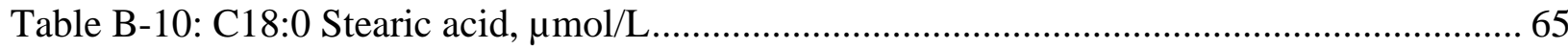

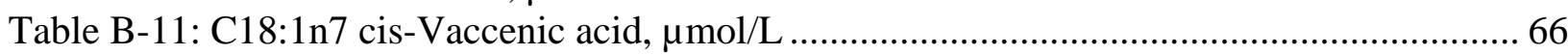

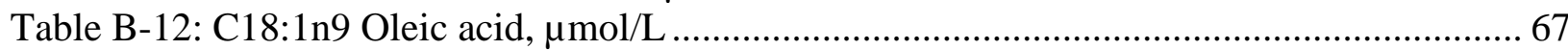

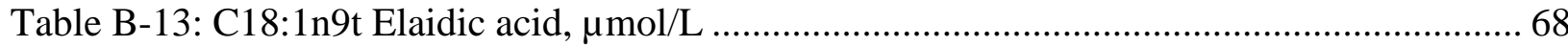

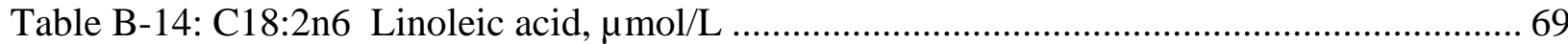

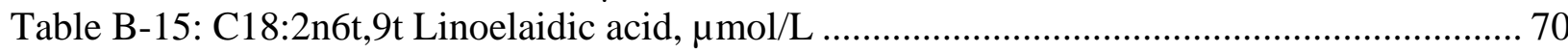

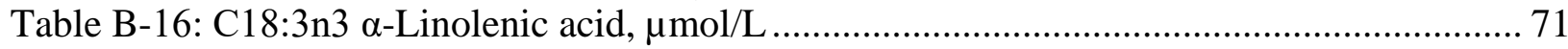

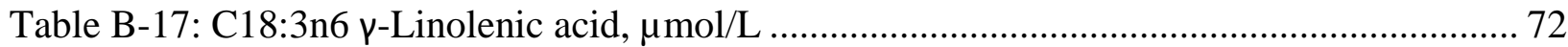

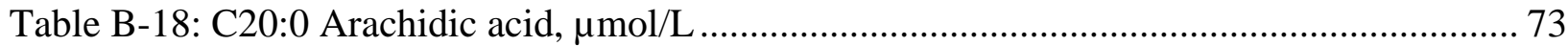

Table B-19: C20:1n9 11-Eicosenoic acid, $\mu$ mol/L …………................................................ 74

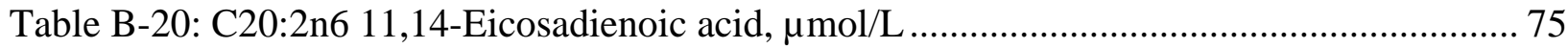

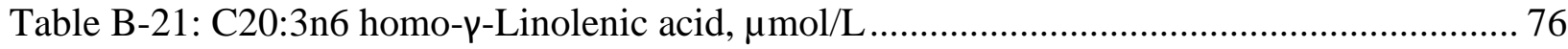

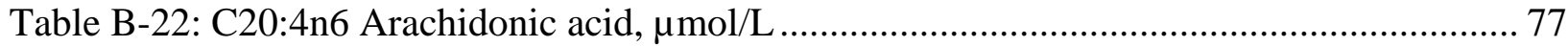

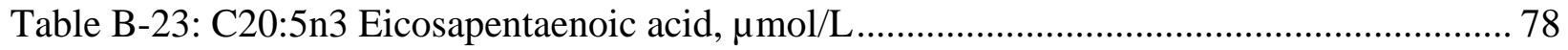

Table B-24: C22:0 Docosanoic acid, $\mu \mathrm{mol} / \mathrm{L}$...................................................................... 79

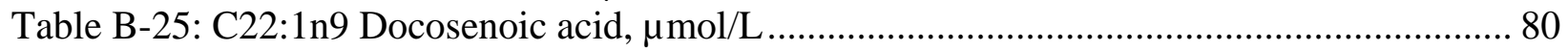

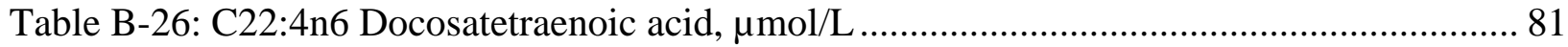

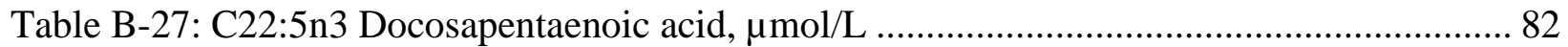

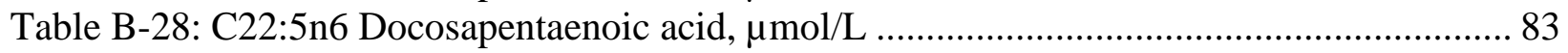

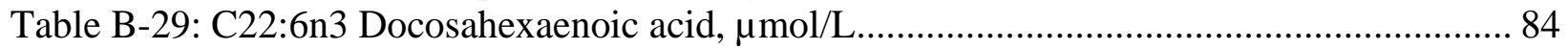

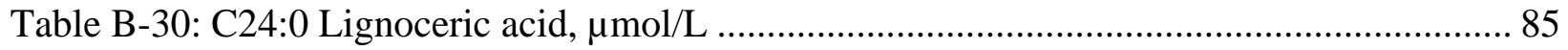

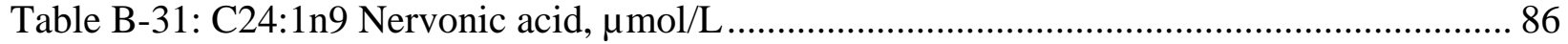

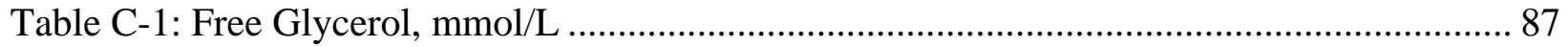

\section{Acronyms Used in Tables}

$\begin{array}{ll}\text { Avg } & \text { Mean } \\ \text { FA } & \text { Fatty acid } \\ \text { FAME } & \text { Fatty acid methyl ester } \\ \text { FFA } & \text { Free fatty acid } \\ \text { Lab } & \text { Participant code } \\ \text { N } & \text { Number of quantitative results } \\ \text { SD } & \text { Standard deviation }\end{array}$


Table B-1: C16:1n5 cis-11-hexadecenoic acid, $\mu \mathrm{mol} / \mathrm{L}$

\begin{tabular}{|c|ccc|cc|c|c|}
\cline { 2 - 7 } \multicolumn{1}{c|}{} & \multicolumn{4}{c|}{ FFA Solutions } & \multicolumn{2}{c|}{ FA/FAME Solution D } \\
\hline Lab & A & B & C & Avg & SD & As FA & As FAME \\
\hline LC05 & & & & & & 51.1 & \\
\hline
\end{tabular}

Table B-2: C16:1n5t trans-11-Hexadecenoic acid, $\mu \mathrm{mol} / \mathrm{L}$

\begin{tabular}{|c|ccc|cc|c|c|}
\cline { 2 - 7 } \multicolumn{1}{c|}{} & \multicolumn{4}{c|}{ FFA Solutions } & \multicolumn{2}{c|}{ FA/FAME Solution D } \\
\hline Lab & A & B & C & Avg & SD & As FA & As FAME \\
\hline LC05 & & & & & & 4.07 & \\
\hline
\end{tabular}

Table B-3: C16:1n10 Sapienic acid, $\mu \mathrm{mol} / \mathrm{L}$

\begin{tabular}{|c|ccc|cc|c|c|}
\cline { 2 - 7 } \multicolumn{1}{c|}{} & \multicolumn{4}{c|}{ FFA Solutions } & \multicolumn{2}{c|}{ FA/FAME Solution D } \\
\hline Lab & A & B & C & Avg & SD & As FA & As FAME \\
\hline LC05 & & & & & & 24.0 & \\
\hline
\end{tabular}

Table B-4: C18:1n7t trans-Vaccenic acid, $\mu \mathrm{mol} / \mathrm{L}$

\begin{tabular}{|c|ccc|cc|c|c|}
\cline { 2 - 7 } \multicolumn{1}{c|}{} & \multicolumn{4}{c|}{ FFA Solutions } & \multicolumn{2}{c|}{ FA/FAME Solution D } \\
\hline Lab & A & B & C & Avg & SD & As FA & As FAME \\
\hline LC12 & & & & & & 30.1 & \\
\hline
\end{tabular}


Table B-5: C14:0 Myristic acid, $\mu \mathrm{mol} / \mathrm{L}$

\begin{tabular}{|c|c|c|c|c|c|c|c|c|c|}
\hline & \multicolumn{5}{|c|}{ FFA Solutions } & \multicolumn{4}{|c|}{ FA/FAME Solution D } \\
\hline Lab & A & $\mathrm{B}$ & $\mathrm{C}$ & Avg & SD & \multicolumn{2}{|c|}{ As FA } & \multicolumn{2}{|c|}{ As FAME } \\
\hline LC01 & & & & & & & 234 & & \\
\hline LC02 & & & & & & & 181 & & \\
\hline LC05 & & & & & & & 156 & & 163 \\
\hline LC07 & & & & & & & 151 & & 168 \\
\hline LC09 & & & & & & & 145 & & \\
\hline LC10 & & & & & & & 133 & & 126 \\
\hline LC11 & & & & & & & & & 148 \\
\hline \multirow[t]{4}{*}{ LC12 } & & & & & & & 143 & & \\
\hline & & & & & & Avg & & Avg & 151 \\
\hline & & & & & & SD & & SD & 34 \\
\hline & & & & & & $\mathrm{N}$ & 7 & $\mathrm{~N}$ & 4 \\
\hline
\end{tabular}
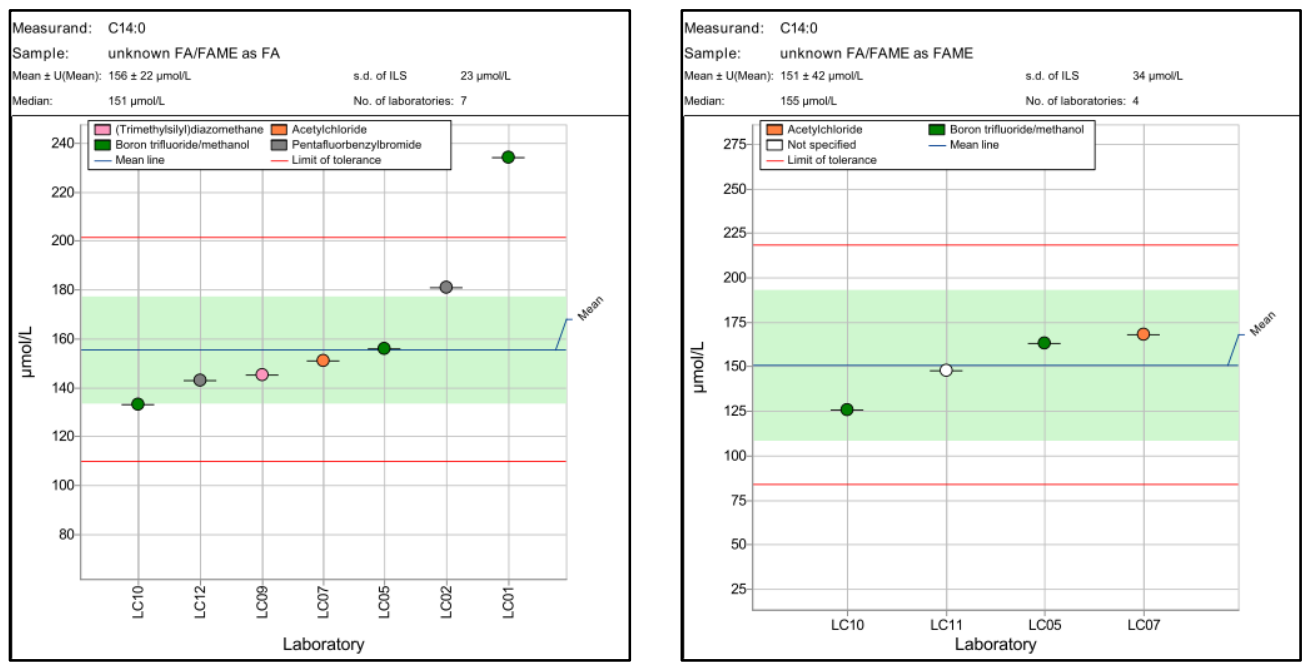
Table B-6: C14:1n5 Myristoleic acid, $\mu \mathrm{mol} / \mathrm{L}$

\begin{tabular}{|c|c|c|c|c|c|c|c|c|c|}
\hline & \multicolumn{5}{|c|}{ FFA Solutions } & \multicolumn{4}{|c|}{ FA/FAME Solution D } \\
\hline Lab & A & B & C & Avg & SD & \multicolumn{2}{|c|}{ As FA } & \multicolumn{2}{|c|}{ As FAME } \\
\hline LC02 & & & & & & & 36 & & \\
\hline LC05 & & & & & & & 26 & & 28 \\
\hline LC09 & & & & & & & 21 & & \\
\hline LC10 & & & & & & & 21 & & 20 \\
\hline LC11 & & & & & & & & & 14 \\
\hline LC12 & & & & & & & 35 & & \\
\hline \multirow[t]{4}{*}{ LC14 } & & & & & & & 26 & & \\
\hline & & & & & & Avg & 27.5 & Avg & 20.5 \\
\hline & & & & & & SD & & SD & 14 \\
\hline & & & & & & $\mathrm{N}$ & & $\mathrm{N}$ & 3 \\
\hline
\end{tabular}
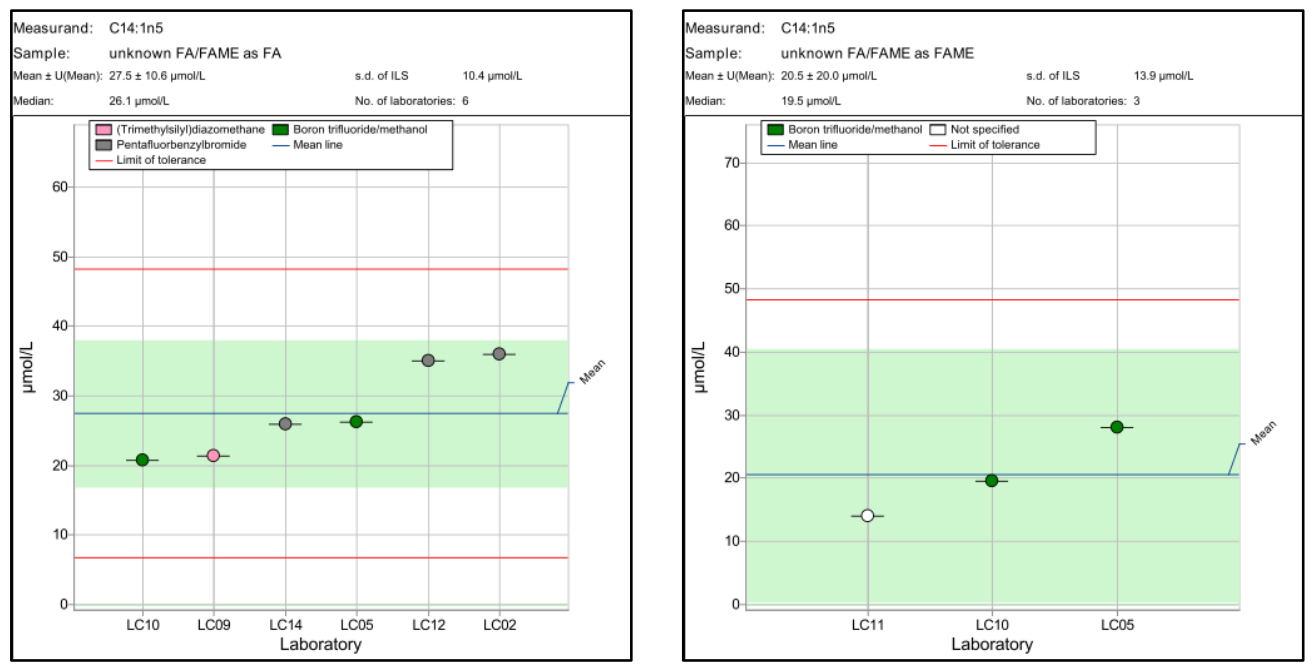
Table B-7: C16:0 Palmitic acid, $\mu \mathrm{mol} / \mathrm{L}$

\begin{tabular}{|c|c|c|c|c|c|c|c|c|c|}
\hline & \multicolumn{5}{|c|}{ FFA Solutions } & \multicolumn{4}{|c|}{ FA/FAME Solution D } \\
\hline $\mathrm{Lab}$ & A & B & $\mathrm{C}$ & Avg & SD & As & & \multicolumn{2}{|c|}{ As FAME } \\
\hline LC01 & & & & & & & 1974 & & \\
\hline LC02 & & & & & & & 2022 & & \\
\hline LC05 & & & & & & & 2173 & & 2186 \\
\hline LC07 & & & & & & & 1895 & & 2091 \\
\hline LC09 & & & & & & & 2397 & & \\
\hline LC10 & & & & & & & 2051 & & 1944 \\
\hline LC11 & & & & & & & & & 2283 \\
\hline \multirow[t]{4}{*}{ LC12 } & & & & & & & 1890 & & \\
\hline & & & & & & Avg & 2047 & Avg & 2126 \\
\hline & & & & & & $\mathrm{SD}$ & & SD & 216 \\
\hline & & & & & & $\mathrm{N}$ & 7 & $\mathrm{~N}$ & 4 \\
\hline
\end{tabular}
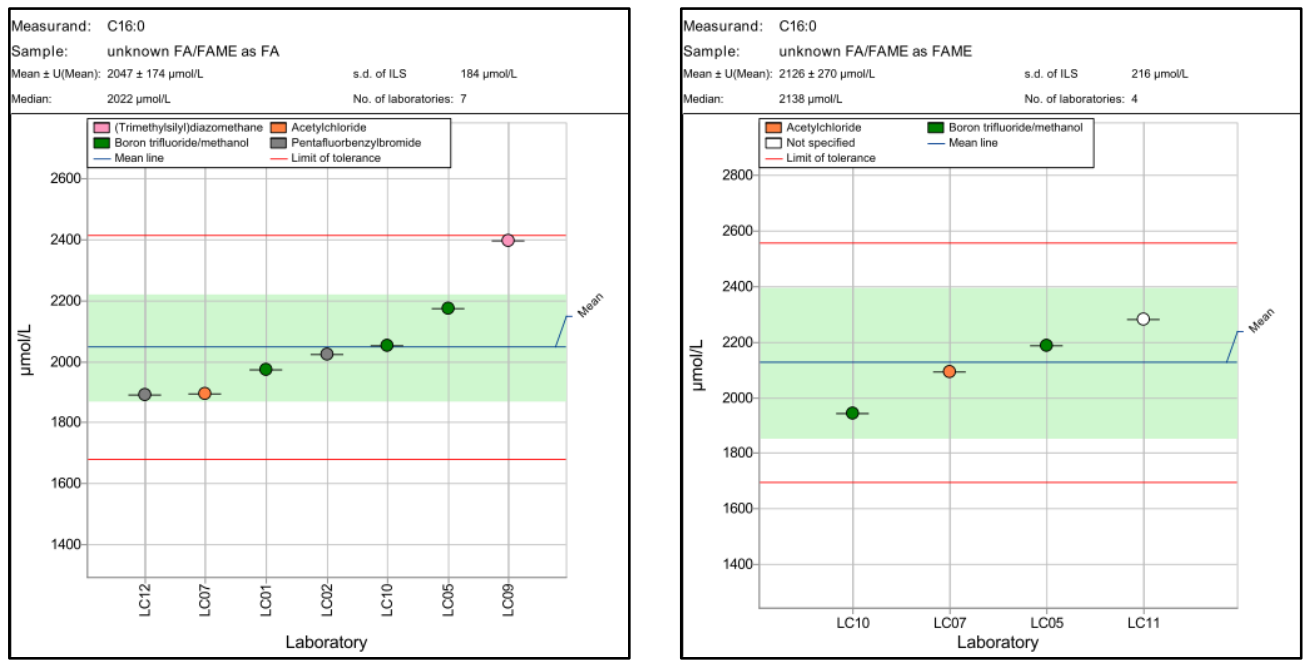
Table B-8: C16:1n7 Palmitoleic acid, $\mu \mathrm{mol} / \mathrm{L}$

\begin{tabular}{|c|c|c|c|c|c|c|c|c|c|}
\hline & \multicolumn{5}{|c|}{ FFA Solutions } & \multicolumn{4}{|c|}{ FA/FAME Solution D } \\
\hline Lab & A & B & $\mathrm{C}$ & Avg & SD & As & & As $\mathrm{F}$ & ME \\
\hline LC01 & & & & & & & 280 & & \\
\hline LC02 & & & & & & & 289 & & \\
\hline LC05 & & & & & & & 323 & & 326 \\
\hline LC07 & & & & & & & 284 & & 315 \\
\hline LC10 & & & & & & & 294 & & 279 \\
\hline LC11 & & & & & & & & & 337 \\
\hline LC12 & & & & & & & 282 & & \\
\hline LC14 & & & & & & & 290 & & \\
\hline & & & & & & Avg & & Avg & \\
\hline & & & & & & SD & & SD & \\
\hline & & & & & & $\mathrm{N}$ & & $\mathrm{N}$ & \\
\hline
\end{tabular}
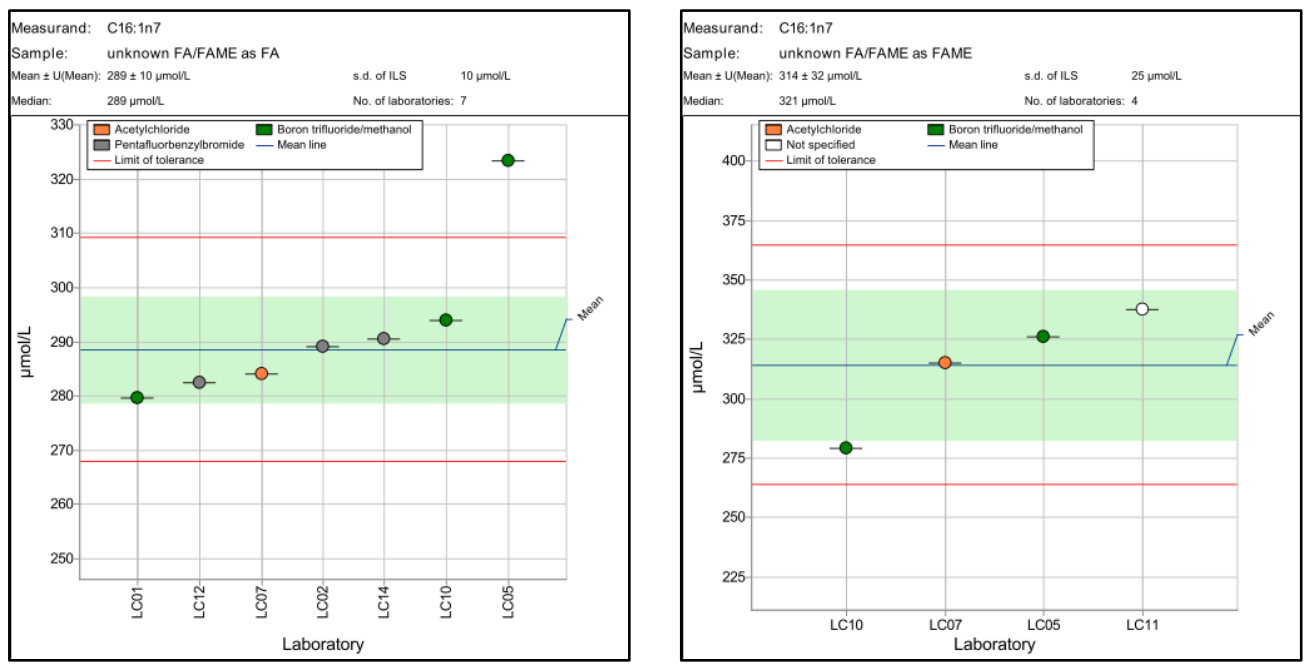
Table B-9: C16:1n7t Palmitelaidic acid, $\mu \mathrm{mol} / \mathrm{L}$

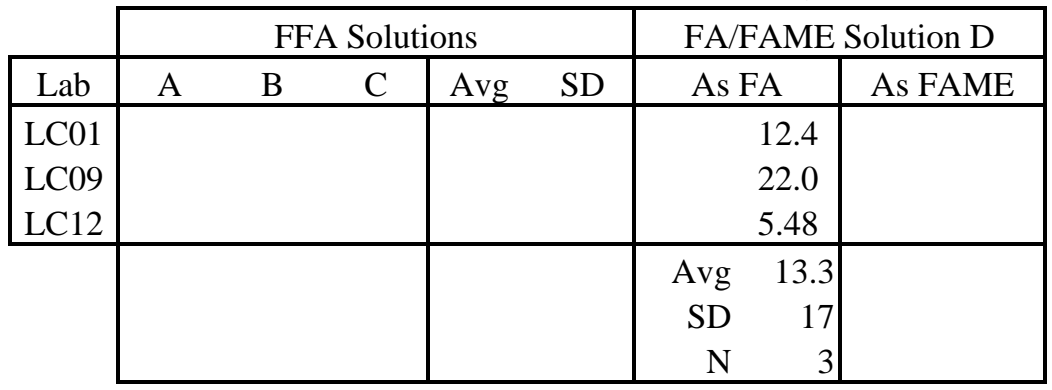

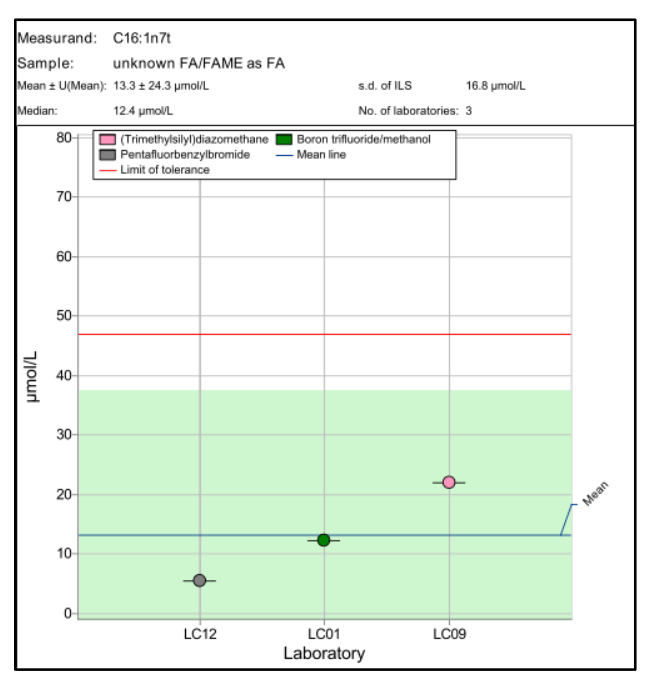


Table B-10: C18:0 Stearic acid, $\mu \mathrm{mol} / \mathrm{L}$

\begin{tabular}{|c|c|c|c|c|c|c|c|c|c|}
\hline \multirow[b]{2}{*}{ Lab } & \multicolumn{5}{|c|}{ FFA Solutions } & \multicolumn{4}{|c|}{ FA/FAME Solution D } \\
\hline & A & B & C & Avg & SD & \multicolumn{2}{|c|}{ As FA } & \multicolumn{2}{|c|}{ As FAME } \\
\hline LC01 & 501 & 508 & 524 & 511 & 12 & \multicolumn{2}{|c|}{550} & \multirow{5}{*}{\multicolumn{2}{|c|}{556}} \\
\hline LC02 & 600 & 609 & 604 & 604 & 4.5 & \multirow{2}{*}{\multicolumn{2}{|c|}{623}} & & \\
\hline LC04 & 506 & 500 & 512 & 506 & 6.1 & & & & \\
\hline LC05 & 572 & 577 & 570 & 573 & 4.0 & \multirow{2}{*}{\multicolumn{2}{|c|}{561}} & & \\
\hline LC06 & 572 & 586 & 567 & 575 & 10 & & & & \\
\hline LC07 & 581 & 586 & 597 & 588 & 8.2 & \multirow{2}{*}{\multicolumn{2}{|c|}{564}} & \multirow{2}{*}{\multicolumn{2}{|c|}{536}} \\
\hline LC08 & 393 & 388 & 391 & 391 & 2.7 & & & & \\
\hline LC09 & 599 & 513 & 515 & 542 & 49 & \multirow{2}{*}{\multicolumn{2}{|c|}{$\begin{array}{l}509 \\
576\end{array}$}} & \multirow{5}{*}{\multicolumn{2}{|c|}{$\begin{array}{l}549 \\
652\end{array}$}} \\
\hline LC10 & 534 & 570 & 547 & 550 & 18.2 & \multirow{3}{*}{\multicolumn{2}{|c|}{576}} & & \\
\hline LC11 & 1709 & 2338 & 1705 & 1918 & 364 & & & & \\
\hline LC12 & 477 & 478 & 481 & 478 & 2.08 & & & & \\
\hline \multirow[t]{4}{*}{ LC14 } & 452 & 456 & 435 & 447 & 11.2 & & 406 & & \\
\hline & & & & Avg & 525 & Avg & 534 & Avg & 559 \\
\hline & & & & SD & 87 & SD & 80 & SD & 29 \\
\hline & & & & $\mathrm{N}$ & 12 & $\mathrm{~N}$ & 8 & $\mathrm{~N}$ & 4 \\
\hline
\end{tabular}
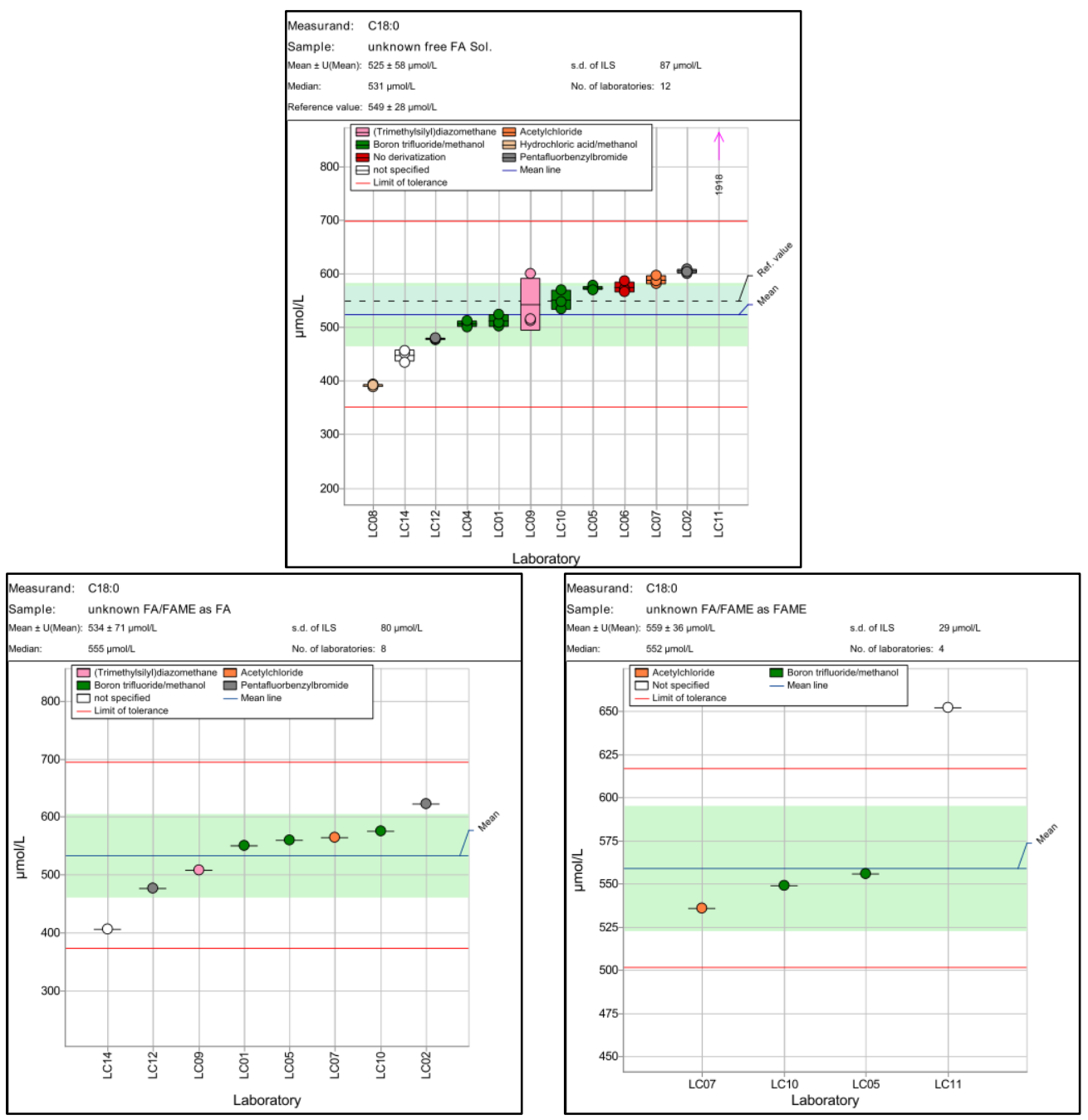
Table B-11: C18:1n7 cis-Vaccenic acid, $\mu \mathrm{mol} / \mathrm{L}$

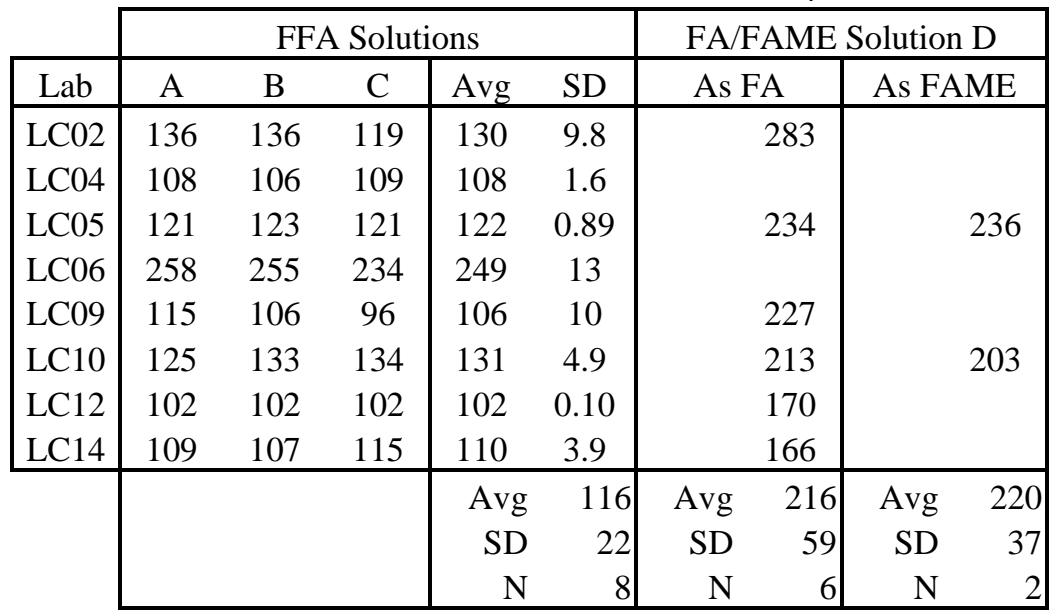
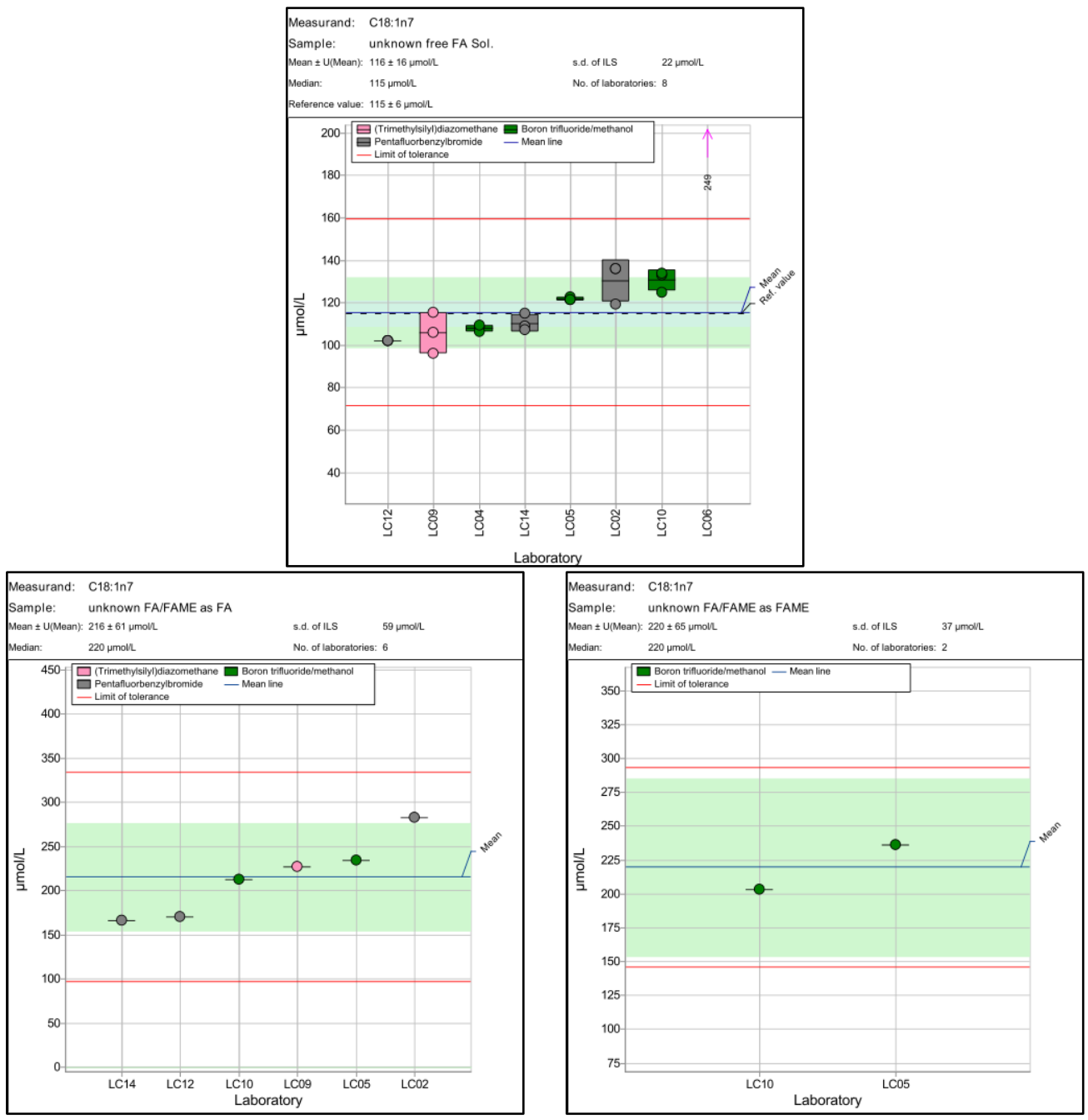
Table B-12: C18:1n9 Oleic acid, $\mu \mathrm{mol} / \mathrm{L}$

\begin{tabular}{|c|c|c|c|c|c|c|c|c|c|}
\hline \multirow[b]{2}{*}{ Lab } & \multicolumn{5}{|c|}{ FFA Solutions } & \multicolumn{4}{|c|}{ FA/FAME Solution D } \\
\hline & A & B & $\mathrm{C}$ & Avg & SD & \multicolumn{2}{|c|}{ As FA } & \multicolumn{2}{|c|}{ As FAME } \\
\hline LC01 & 1215 & 1218 & 1227 & 1220 & 6.5 & \multicolumn{2}{|r|}{1592} & \multirow{5}{*}{\multicolumn{2}{|c|}{1816}} \\
\hline LC02 & 1303 & 1319 & 1274 & 1299 & 23 & \multirow{2}{*}{\multicolumn{2}{|c|}{1711}} & & \\
\hline LC04 & 1183 & 1165 & 1178 & 1175 & 9.4 & & & & \\
\hline LC05 & 1379 & 1398 & 1398 & 1391 & 10.9 & \multirow{2}{*}{\multicolumn{2}{|c|}{1805}} & & \\
\hline LC06 & 1477 & 1414 & 1415 & 1436 & 36 & & & & \\
\hline LC07 & 1306 & 1289 & 1319 & 1305 & 15 & \multirow{2}{*}{\multicolumn{2}{|c|}{1482}} & \multirow{2}{*}{\multicolumn{2}{|c|}{1654}} \\
\hline LC08 & 683 & 676 & 685 & 682 & 4.8 & & & & \\
\hline LC09 & 1885 & 1606 & 1562 & 1685 & 175 & \multirow{2}{*}{\multicolumn{2}{|c|}{$\begin{array}{l}2102 \\
1685\end{array}$}} & \multirow{5}{*}{\multicolumn{2}{|c|}{$\begin{array}{l}1605 \\
2179\end{array}$}} \\
\hline LC10 & 1277 & 1273 & 1269 & 1273 & 4.0 & & & & \\
\hline LC11 & 2393 & 2304 & 2436 & 2377 & 67 & & & & \\
\hline LC12 & 1150 & 1150 & 1150 & 1150 & 0.0 & \multicolumn{2}{|r|}{1400} & & \\
\hline \multirow[t]{4}{*}{ LC14 } & 1292 & 1282 & 1282 & 1285 & 6.0 & & 1481 & & \\
\hline & & & & Avg & 1282 & Avg & 1647 & Avg & 1814 \\
\hline & & & & SD & 217 & SD & 246 & SD & 360 \\
\hline & & & & $\mathrm{N}$ & 12 & $\mathrm{~N}$ & 8 & $\mathrm{~N}$ & 4 \\
\hline
\end{tabular}
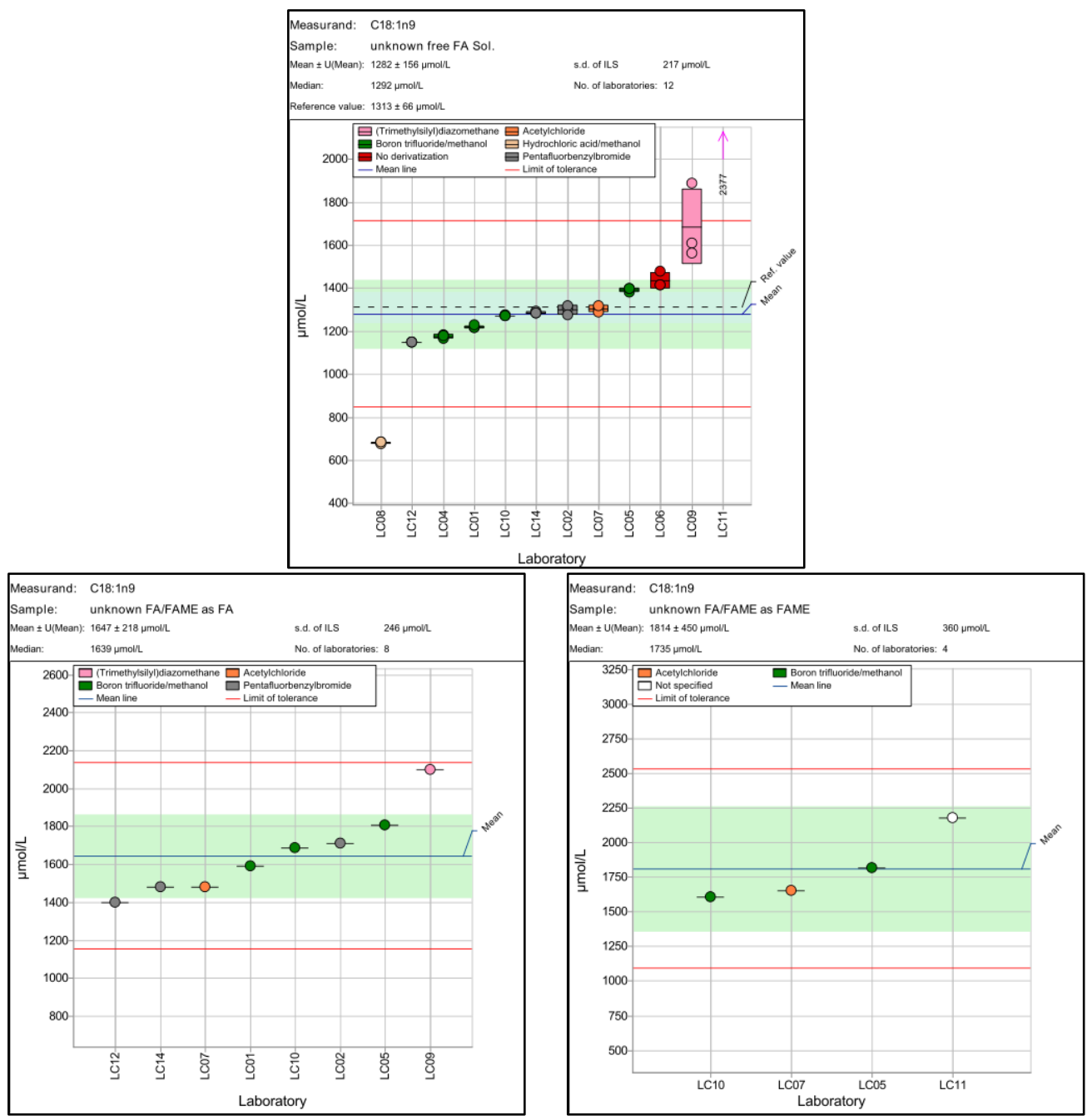
Table B-13: C18:1n9t Elaidic acid, $\mu \mathrm{mol} / \mathrm{L}$

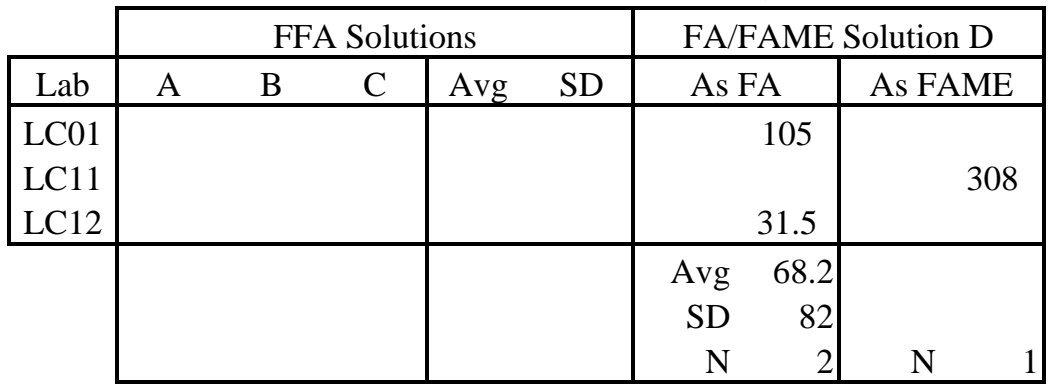

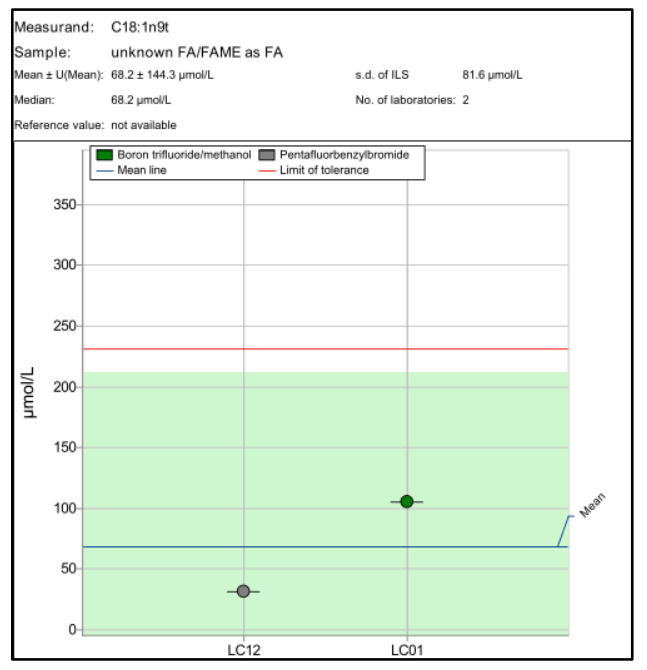


Table B-14: C18:2n6 Linoleic acid, $\mu \mathrm{mol} / \mathrm{L}$

\begin{tabular}{|c|c|c|c|c|c|c|c|c|c|}
\hline \multirow[b]{2}{*}{ Lab } & \multicolumn{5}{|c|}{ FFA Solutions } & \multicolumn{4}{|c|}{ FA/FAME Solution D } \\
\hline & A & B & $\mathrm{C}$ & Avg & SD & As & & \multicolumn{2}{|c|}{ As FAME } \\
\hline LC01 & 2241 & 2250 & 2266 & 2252 & 13 & \multicolumn{2}{|r|}{2017} & \multirow{5}{*}{\multicolumn{2}{|c|}{2250}} \\
\hline LC02 & 2362 & 2374 & 2342 & 2359 & 16 & \multirow{2}{*}{\multicolumn{2}{|c|}{2144}} & & \\
\hline LC04 & 2186 & 2153 & 2152 & 2164 & 20 & & & & \\
\hline LC05 & 2607 & 2635 & 2602 & 2615 & 18 & \multirow{2}{*}{\multicolumn{2}{|c|}{2233}} & & \\
\hline LC06 & 2530 & 2581 & 2620 & 2577 & 45 & & & & \\
\hline LC07 & 2447 & 2416 & 2466 & 2443 & 25 & \multirow{2}{*}{\multicolumn{2}{|c|}{1966}} & \multirow{3}{*}{\multicolumn{2}{|c|}{2176}} \\
\hline LC08 & 1370 & 1351 & 1366 & 1363 & 9.9 & & & & \\
\hline LC09 & 3706 & 3153 & 3076 & 3312 & 343 & \multirow{2}{*}{\multicolumn{2}{|c|}{$\begin{array}{l}2567 \\
2068\end{array}$}} & & \\
\hline LC10 & 2459 & 2404 & 2390 & 2418 & 36 & & & \multirow{4}{*}{\multicolumn{2}{|c|}{$\begin{array}{l}1970 \\
2558\end{array}$}} \\
\hline LC11 & 4122 & 3661 & 4114 & 3965 & 264 & & & & \\
\hline LC12 & 2160 & 2160 & 2170 & 2163 & 5.8 & \multicolumn{2}{|r|}{1840} & & \\
\hline \multirow[t]{4}{*}{ LC14 } & 2351 & 2313 & 2308 & 2324 & 23.7 & & 1964 & & \\
\hline & & & & Avg & 2375 & Avg & 2082 & Avg & 2239 \\
\hline & & & & $\mathrm{SD}$ & 367 & SD & 229 & SD & 457 \\
\hline & & & & $\mathrm{N}$ & 12 & $\mathrm{~N}$ & & $\mathrm{~N}$ & 4 \\
\hline
\end{tabular}
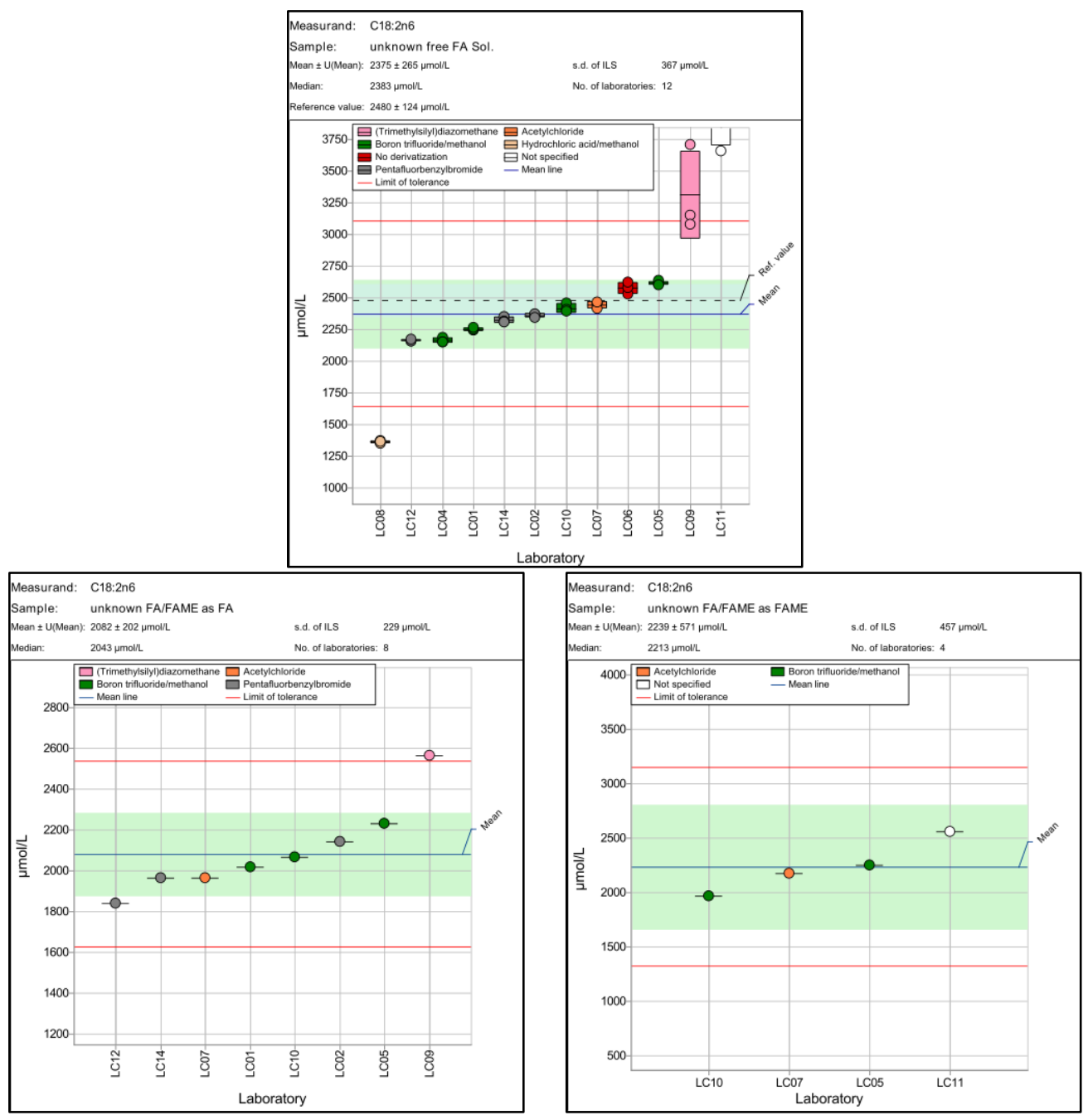
Table B-15: C18:2n6t,9t Linoelaidic acid, $\mu \mathrm{mol} / \mathrm{L}$

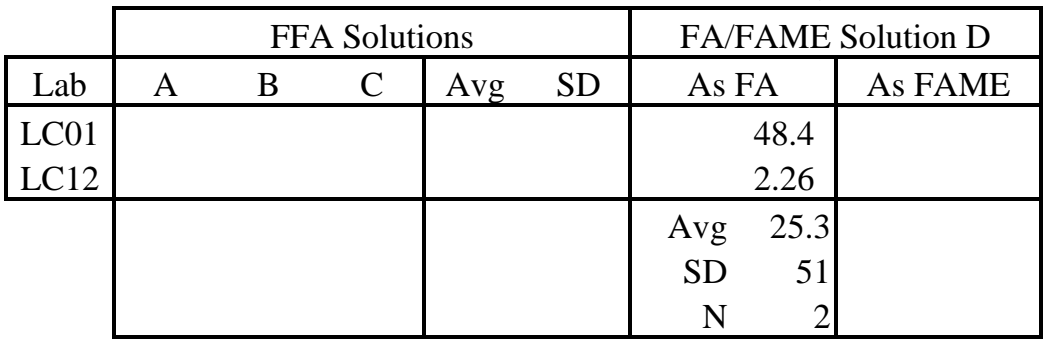

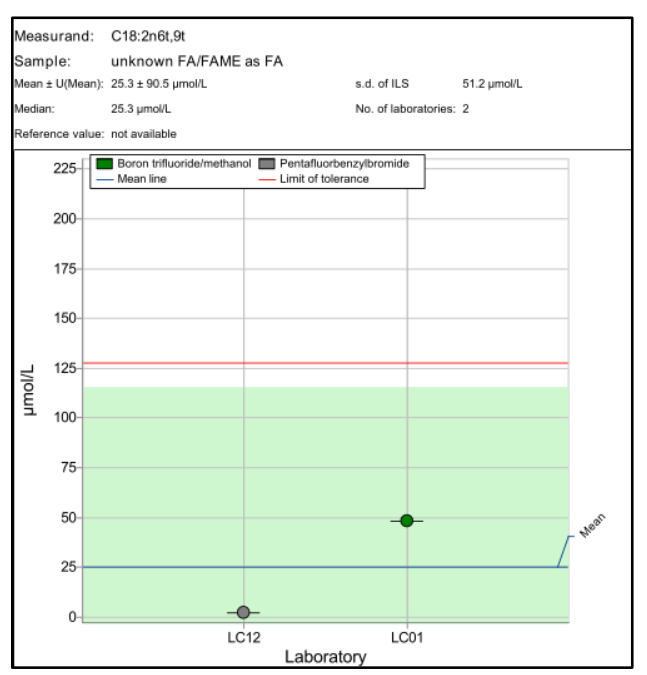


Table B-16: C18:3n3 $\alpha$-Linolenic acid, $\mu \mathrm{mol} / \mathrm{L}$

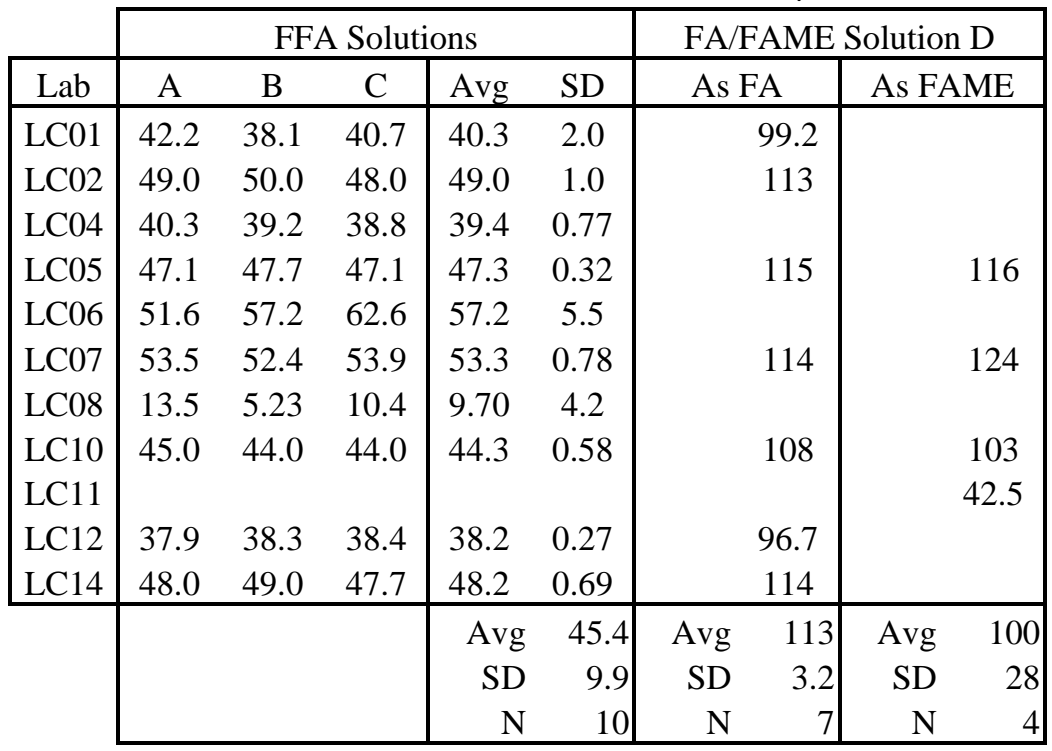
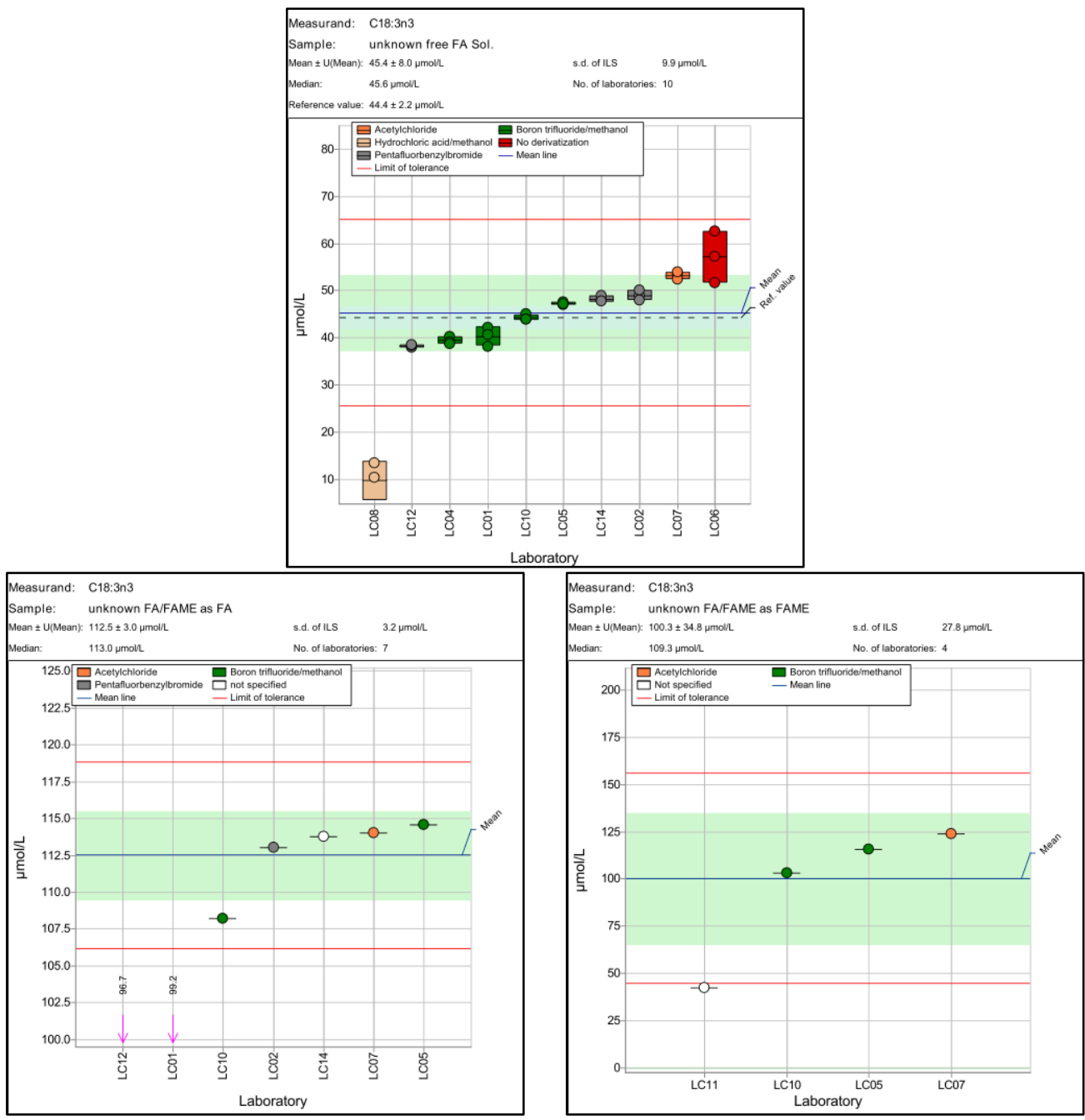


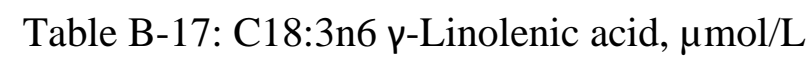

\begin{tabular}{|c|c|c|c|c|c|c|c|c|c|}
\hline & \multicolumn{5}{|c|}{ FFA Solutions } & \multicolumn{4}{|c|}{ FA/FAME Solution D } \\
\hline Lab & A & B & $\mathrm{C}$ & Avg & SD & & & As FA & $\mathrm{ME}$ \\
\hline LC01 & & & & & & & 52.5 & & \\
\hline LC02 & & & & & & & 63.0 & & \\
\hline LC05 & & & & & & & 56.8 & & 57.6 \\
\hline LC07 & & & & & & & 55.4 & & 61.9 \\
\hline LC09 & & & & & & & 47.9 & & \\
\hline LC10 & & & & & & & 53.9 & & 51.3 \\
\hline LC11 & & & & & & & & & 0.0 \\
\hline LC12 & & & & & & & 49.2 & & \\
\hline LC14 & & & & & & & 52.0 & & \\
\hline & & & & & & Avg & & Avg & 53.8 \\
\hline & & & & & & SD & & SD & 14 \\
\hline & & & & & & $\mathrm{N}$ & 8 & $\mathrm{~N}$ & 4 \\
\hline
\end{tabular}
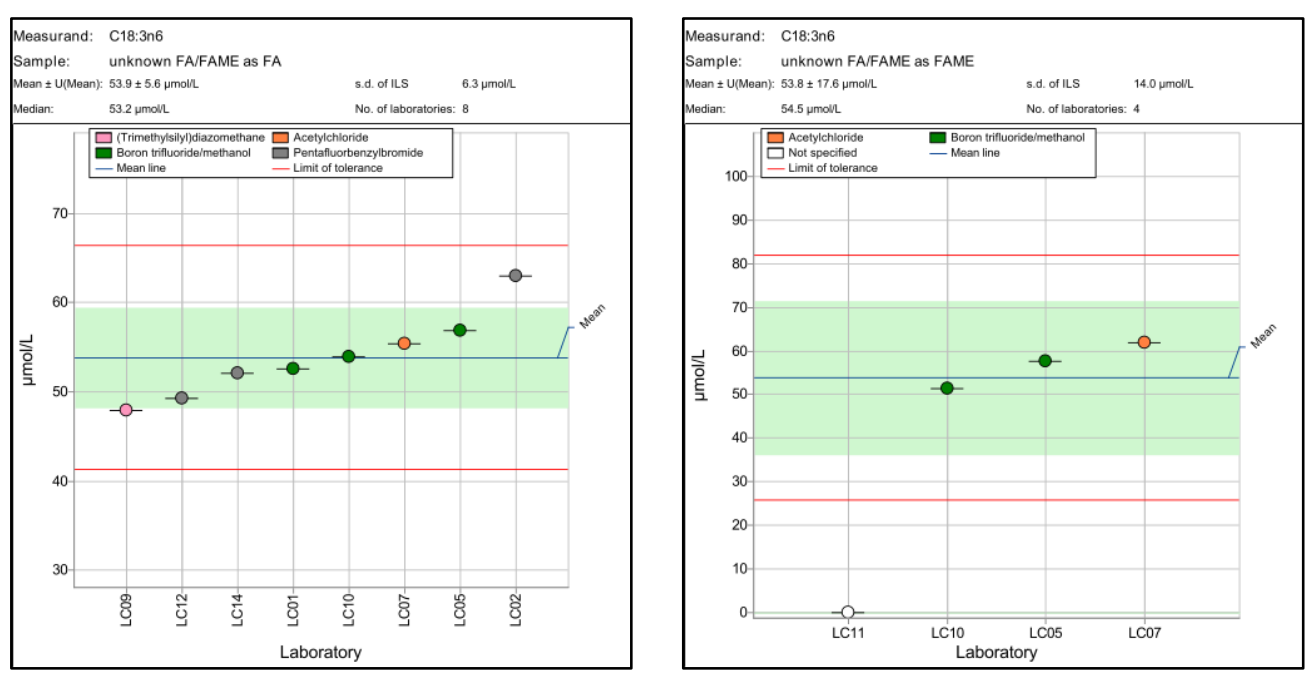
Table B-18: C20:0 Arachidic acid, $\mu \mathrm{mol} / \mathrm{L}$

\begin{tabular}{|c|c|c|c|c|c|c|c|c|c|}
\hline \multirow[b]{2}{*}{$\mathrm{Lab}$} & \multicolumn{5}{|c|}{ FFA Solutions } & \multicolumn{4}{|c|}{ FA/FAME Solution D } \\
\hline & A & B & $\mathrm{C}$ & Avg & SD & \multicolumn{2}{|c|}{ As FA } & \multicolumn{2}{|c|}{ As FAME } \\
\hline LC01 & & & & & & & 55.2 & & \\
\hline LC02 & & & & & & & 61.0 & & \\
\hline LC05 & & & & & & & 55.8 & & 56.0 \\
\hline LC07 & & & & & & & 50.8 & & 54.8 \\
\hline LC10 & & & & & & & 56.0 & & 53.1 \\
\hline LC11 & & & & & & & & & 47.5 \\
\hline LC12 & & & & & & & 49.5 & & \\
\hline \multirow[t]{4}{*}{ LC14 } & & & & & & & 45.7 & & \\
\hline & & & & & & Avg & 53.4 & Avg & 52.8 \\
\hline & & & & & & SD & 9.4 & $\mathrm{SD}$ & 3.8 \\
\hline & & & & & & $\mathrm{N}$ & 7 & $\mathrm{~N}$ & 4 \\
\hline
\end{tabular}
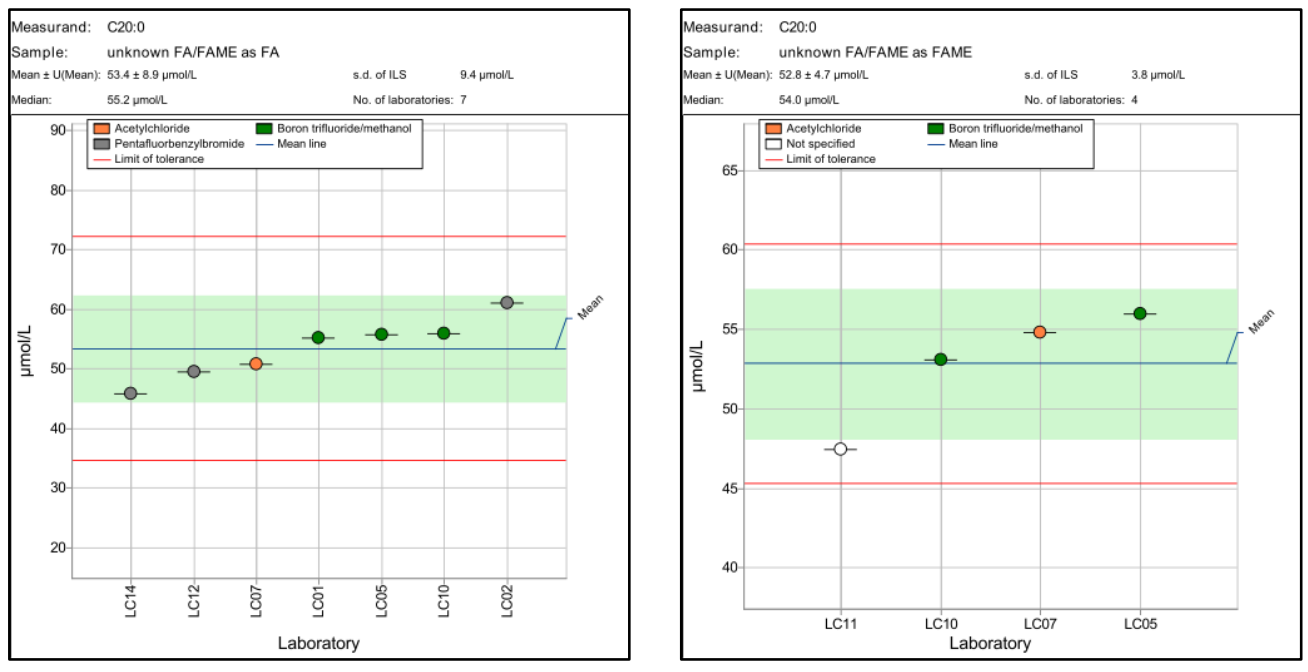
Table B-19: C20:1n9 11-Eicosenoic acid, $\mu \mathrm{mol} / \mathrm{L}$

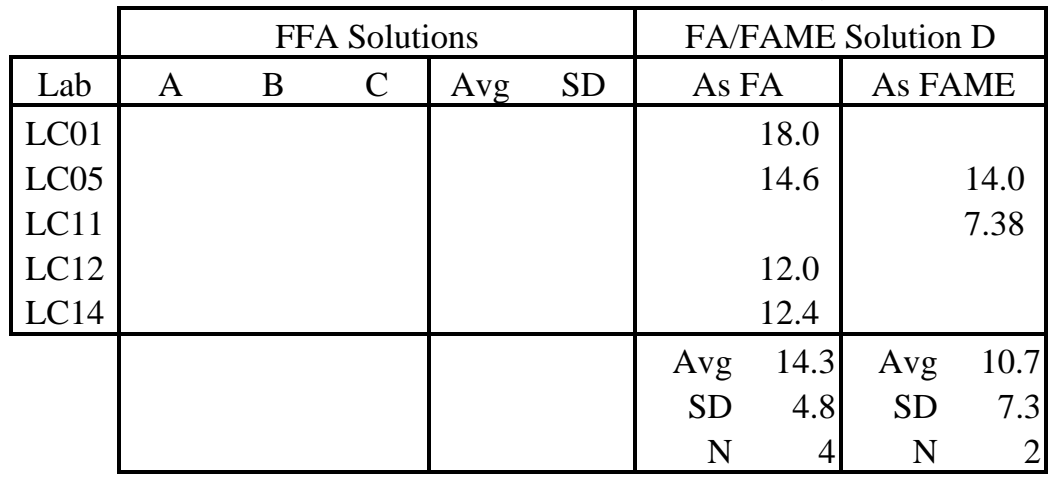
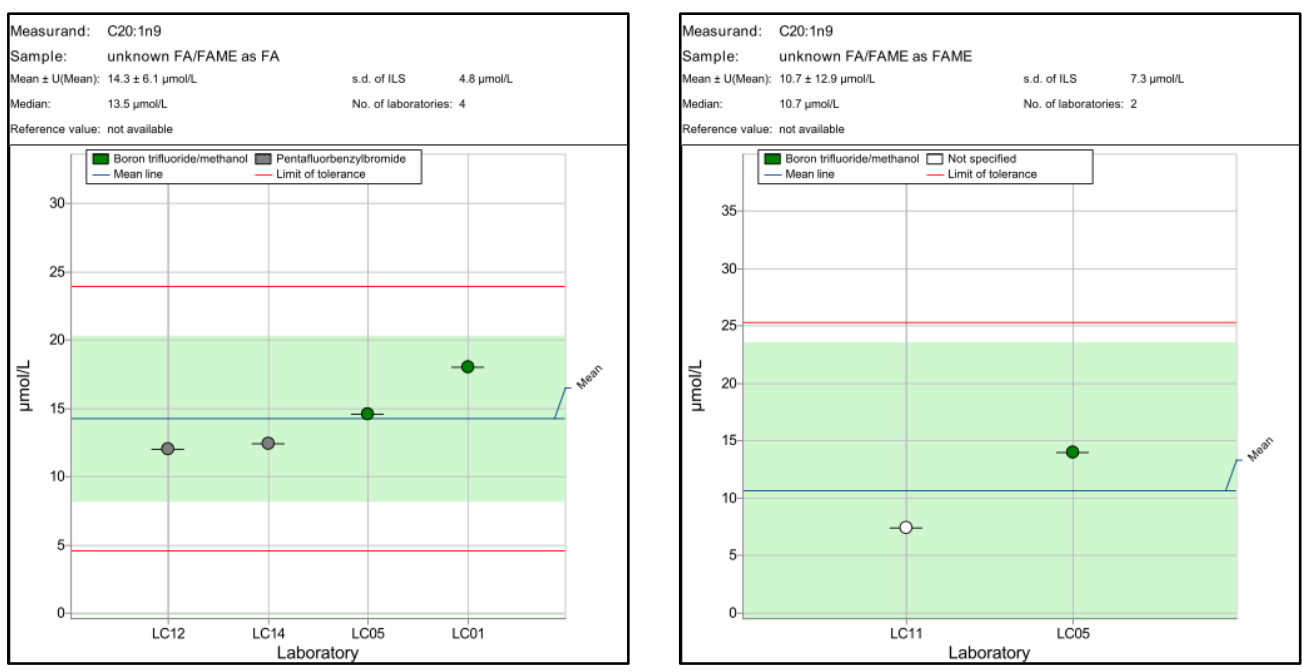
Table B-20: C20:2n6 11,14-Eicosadienoic acid, $\mu \mathrm{mol} / \mathrm{L}$

\begin{tabular}{|c|c|c|c|c|c|c|c|c|c|}
\hline & \multicolumn{5}{|c|}{ FFA Solutions } & \multicolumn{4}{|c|}{ FA/FAME Solution D } \\
\hline $\mathrm{Lab}$ & A & B & C & Avg & SD & \multicolumn{2}{|c|}{ As FA } & \multicolumn{2}{|c|}{ As FAME } \\
\hline LC01 & & & & & & & 15.3 & & \\
\hline LC05 & & & & & & & 13.6 & & 13.7 \\
\hline LC09 & & & & & & & 4.43 & & \\
\hline LC10 & & & & & & & 13.3 & & 12.7 \\
\hline LC11 & & & & & & & & & 6.70 \\
\hline LC12 & & & & & & & 11.8 & & \\
\hline \multirow[t]{4}{*}{ LC14 } & & & & & & & 12.0 & & \\
\hline & & & & & & Avg & 12.2 & Avg & \\
\hline & & & & & & SD & & SD & 5.0 \\
\hline & & & & & & $\mathrm{N}$ & 6 & $\mathrm{~N}$ & 3 \\
\hline
\end{tabular}
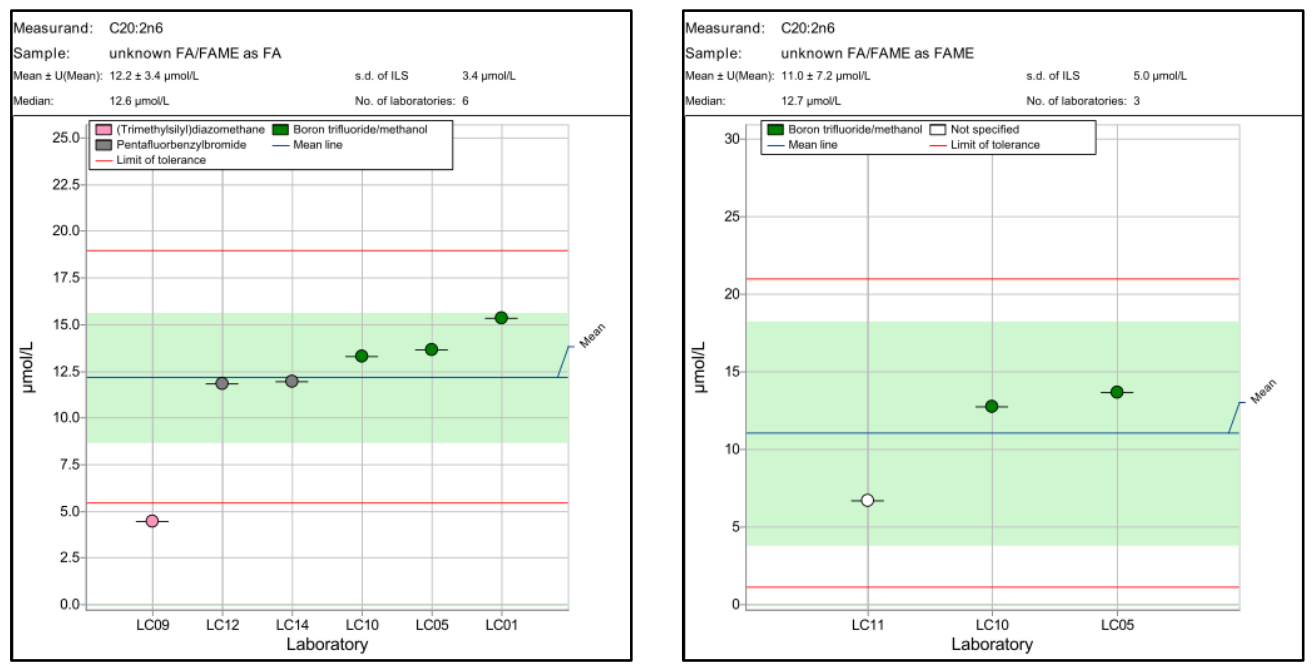
Table B-21: C20:3n6 homo- $\psi$-Linolenic acid, $\mu \mathrm{mol} / \mathrm{L}$

\begin{tabular}{|c|c|c|c|c|c|c|c|c|c|}
\hline & \multicolumn{5}{|c|}{ FFA Solutions } & \multicolumn{4}{|c|}{ FA/FAME Solution D } \\
\hline $\mathrm{Lab}$ & A & B & C & Avg & SD & As & & \multicolumn{2}{|c|}{ As FAME } \\
\hline LC01 & & & & & & & 72.1 & & \\
\hline LC02 & & & & & & & 58.0 & & \\
\hline LC05 & & & & & & & 72.2 & & 72.5 \\
\hline LC07 & & & & & & & 75.8 & & 83.5 \\
\hline LC09 & & & & & & & 32.7 & & \\
\hline LC10 & & & & & & & 68.5 & & 65.5 \\
\hline LC11 & & & & & & & & & 72.7 \\
\hline LC12 & & & & & & & 61.3 & & \\
\hline \multirow[t]{4}{*}{ LC14 } & & & & & & & 63.5 & & \\
\hline & & & & & & & & Avg & 73.6 \\
\hline & & & & & & $\mathrm{SD}$ & 9.7 & SD & 16 \\
\hline & & & & & & $\mathrm{N}$ & 8 & $\mathrm{~N}$ & 4 \\
\hline
\end{tabular}
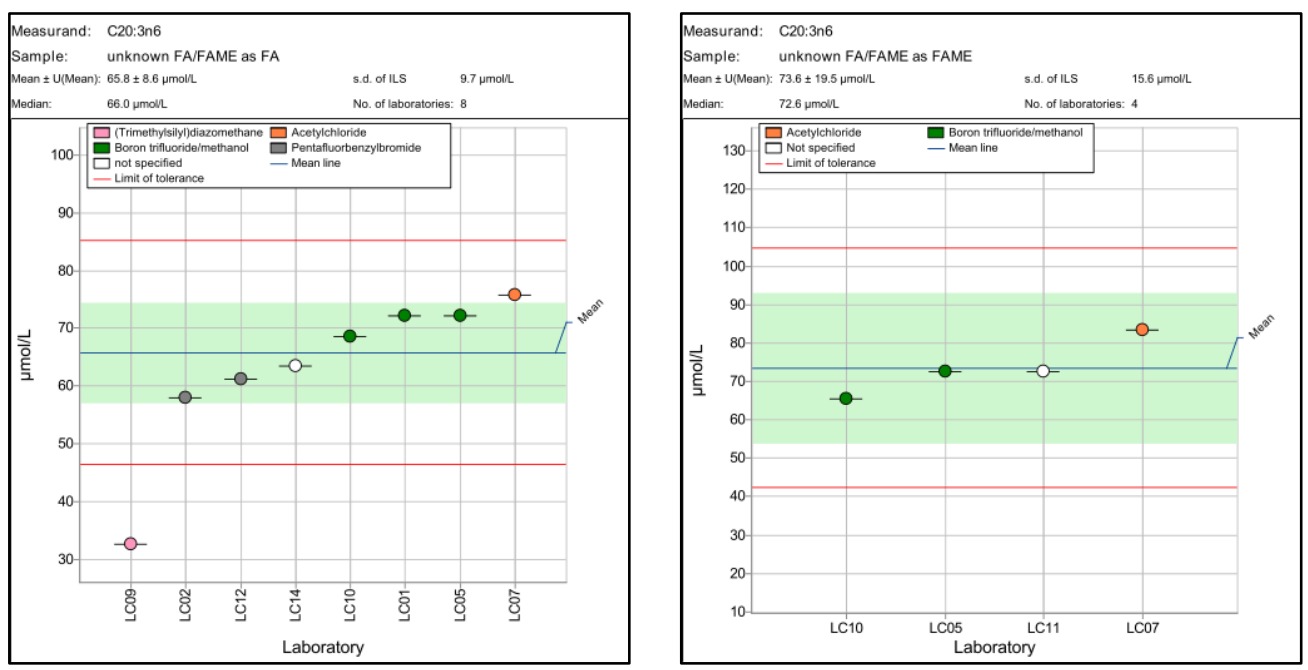
Table B-22: C20:4n6 Arachidonic acid, $\mu \mathrm{mol} / \mathrm{L}$

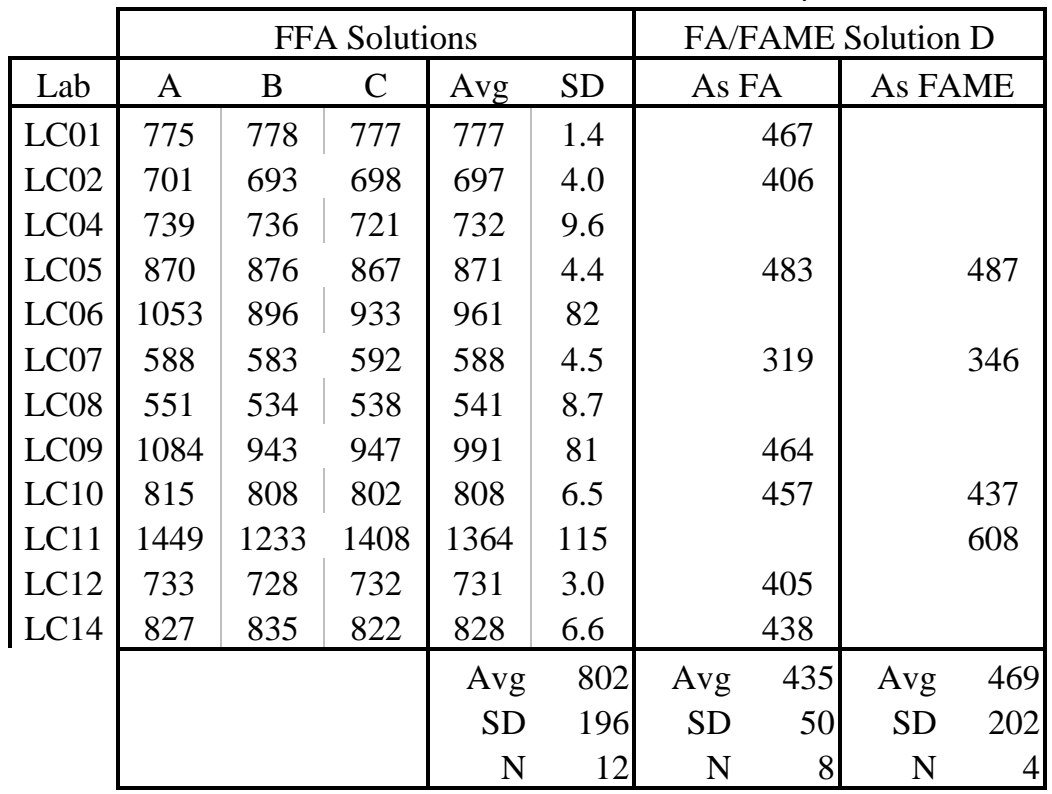
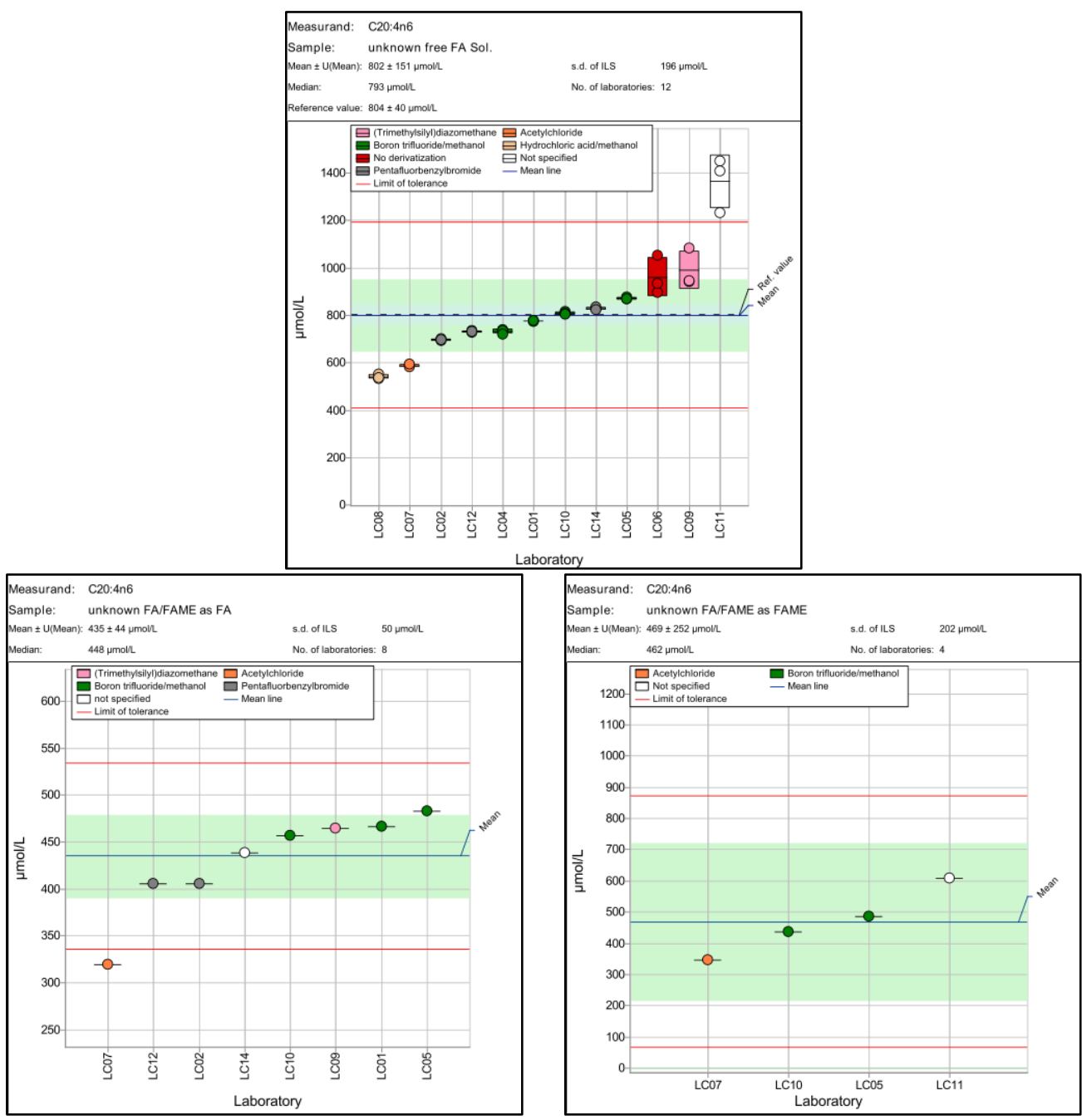
Table B-23: C20:5n3 Eicosapentaenoic acid, $\mu \mathrm{mol} / \mathrm{L}$

\begin{tabular}{|c|c|c|c|c|c|c|c|c|c|}
\hline \multirow[b]{2}{*}{$\mathrm{Lab}$} & \multicolumn{5}{|c|}{ FFA Solutions } & \multicolumn{4}{|c|}{ FA/FAME Solution D } \\
\hline & A & B & $\mathrm{C}$ & Avg & SD & As & & \multicolumn{2}{|c|}{ As FAME } \\
\hline LC01 & 32.9 & 34.1 & 39.7 & 35.6 & 3.7 & \multicolumn{2}{|c|}{184} & \multirow{4}{*}{\multicolumn{2}{|c|}{204}} \\
\hline LC02 & 40.0 & 39.0 & 40.0 & 39.7 & 0.58 & \multirow{2}{*}{\multicolumn{2}{|c|}{206}} & & \\
\hline LC04 & 32.0 & 32.2 & 31.3 & 31.8 & 0.44 & & & & \\
\hline LC05 & 38.1 & 38.0 & 37.2 & 37.8 & 0.47 & \multicolumn{2}{|c|}{202} & & \\
\hline LC07 & 44.3 & 45.3 & 46.3 & 45.3 & 1.0 & \multicolumn{2}{|c|}{225} & \multirow{2}{*}{\multicolumn{2}{|c|}{254}} \\
\hline LC08 & 23.5 & 23.3 & 20.3 & 22.4 & 1.8 & & & & \\
\hline LC09 & 19.4 & 17.2 & 17.1 & 17.9 & 1.3 & \multicolumn{2}{|r|}{143} & \multirow{4}{*}{\multicolumn{2}{|c|}{$\begin{array}{l}187 \\
247\end{array}$}} \\
\hline LC10 & 39.3 & 41.3 & 41.0 & 40.5 & 1.08 & & 196 & & \\
\hline LC11 & & & & & & & & & \\
\hline \multirow[t]{4}{*}{ LC12 } & 28.5 & 29.1 & 28.7 & 28.8 & 0.32 & & 172 & & \\
\hline & & & & Avg & 33.3 & Avg & 190 & Avg & 223 \\
\hline & & & & SD & 12 & SD & 36 & SD & 38 \\
\hline & & & & $\mathrm{N}$ & $y$ & $\mathrm{~N}$ & & $\mathrm{~N}$ & \\
\hline
\end{tabular}
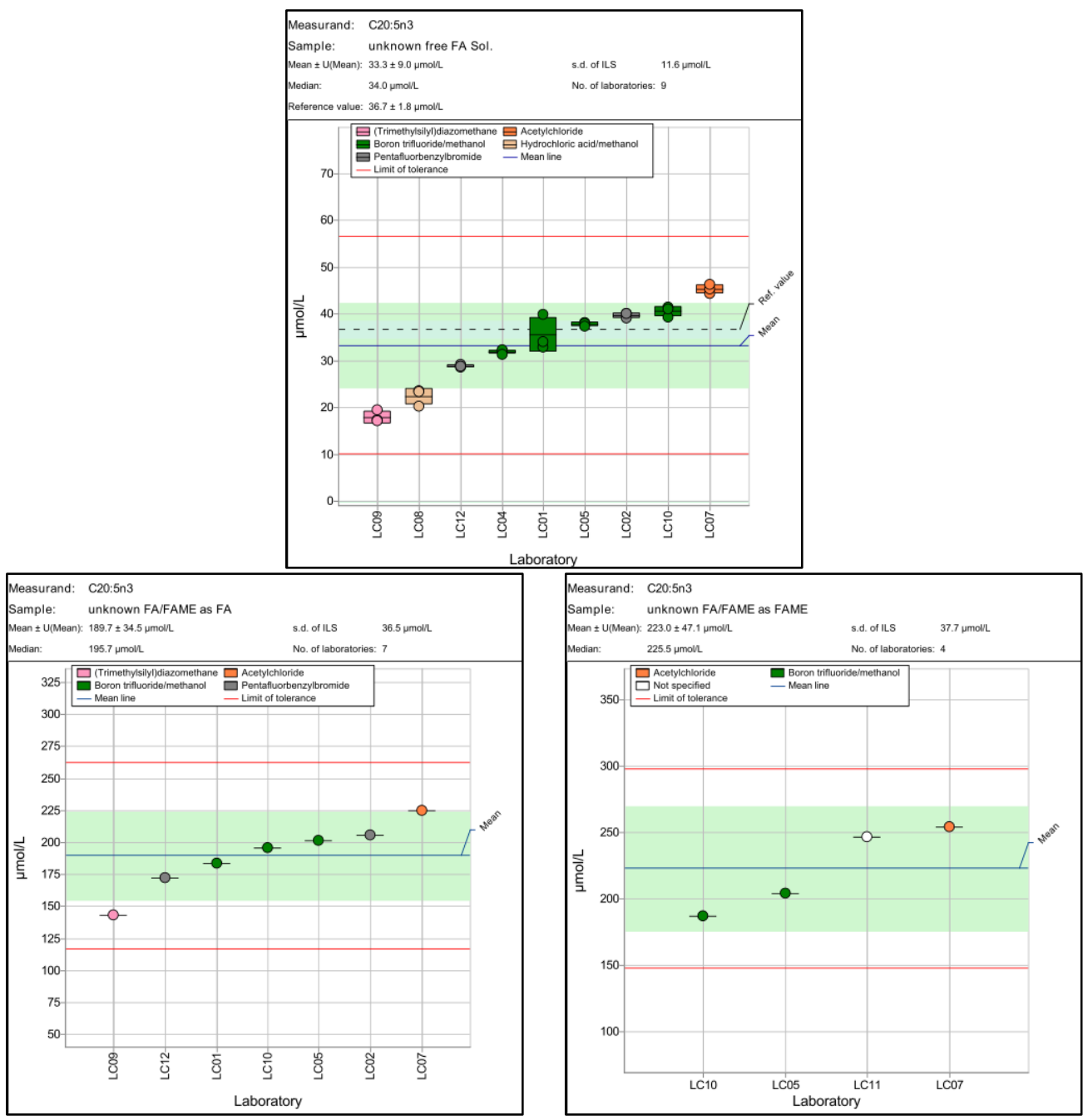
Table B-24: C22:0 Docosanoic acid, $\mu \mathrm{mol} / \mathrm{L}$

\begin{tabular}{|c|c|c|c|c|c|c|c|c|c|}
\hline & \multicolumn{5}{|c|}{ FFA Solutions } & \multicolumn{4}{|c|}{ FA/FAME Solution D } \\
\hline Lab & A & B & $\mathrm{C}$ & Avg & SD & \multicolumn{2}{|c|}{ As FA } & \multicolumn{2}{|c|}{ As FAME } \\
\hline LC01 & & & & & & & 58.2 & & \\
\hline LC02 & & & & & & & 55.1 & & \\
\hline LC05 & & & & & & & 54.7 & & 55.5 \\
\hline LC07 & & & & & & & 48.8 & & 55.0 \\
\hline LC10 & & & & & & & 51.5 & & 49.4 \\
\hline LC11 & & & & & & & & & 36.3 \\
\hline \multirow[t]{4}{*}{ LC12 } & & & & & & & 49.9 & & \\
\hline & & & & & & Avg & 53.0 & Avg & 49.0 \\
\hline & & & & & & $\mathrm{SD}$ & 6.2 & SD & 12 \\
\hline & & & & & & $\mathrm{N}$ & 6 & $\mathrm{~N}$ & 4 \\
\hline
\end{tabular}
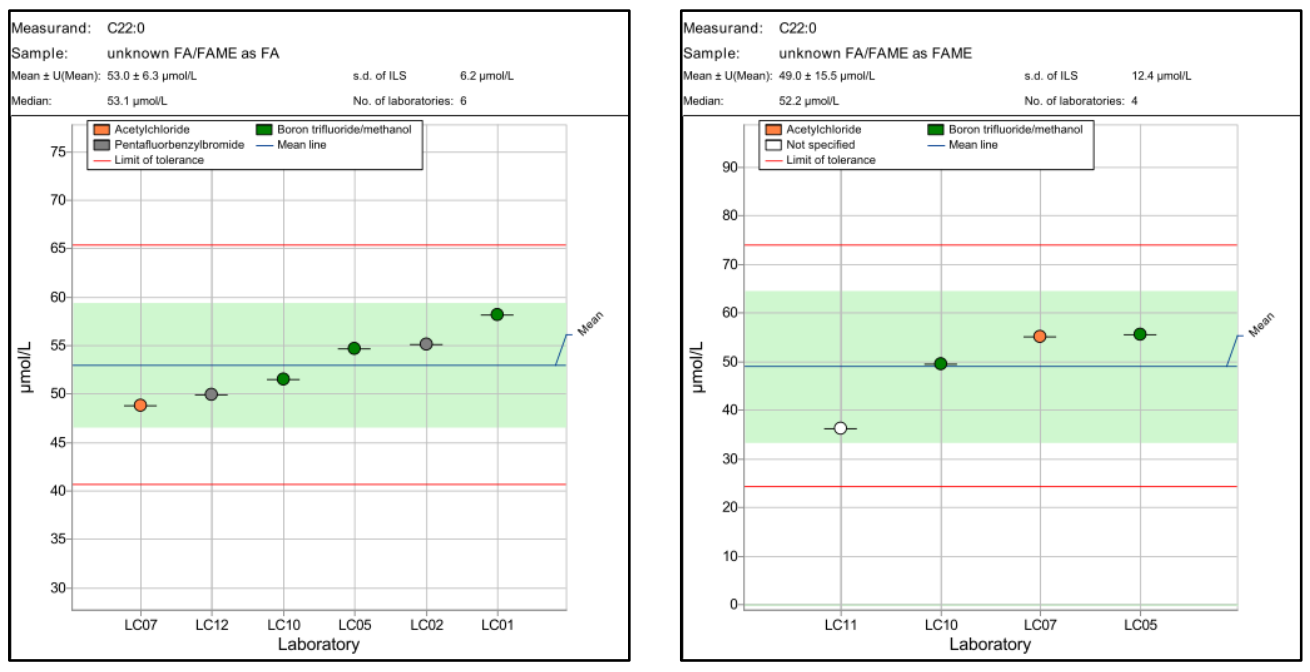
Table B-25: C22:1n9 Docosenoic acid, $\mu \mathrm{mol} / \mathrm{L}$

\begin{tabular}{|c|c|c|c|c|c|c|c|c|c|}
\hline & \multicolumn{5}{|c|}{ FFA Solutions } & \multicolumn{4}{|c|}{ FA/FAME Solution D } \\
\hline Lab & A & B & C & Avg & SD & \multicolumn{2}{|c|}{ As FA } & \multicolumn{2}{|c|}{ As FAME } \\
\hline LC01 & & & & & & & & & \\
\hline LC02 & & & & & & & 28.0 & & \\
\hline LC05 & & & & & & & 20.1 & & 14.5 \\
\hline LC10 & & & & & & & 16.9 & & 16.2 \\
\hline \multirow[t]{4}{*}{ LC11 } & & & & & & & & & 5.26 \\
\hline & & & & & & Avg & 21.7 & Avg & \\
\hline & & & & & & SD & & SD & 7.9 \\
\hline & & & & & & $\mathrm{N}$ & 3 & $\mathrm{~N}$ & \\
\hline
\end{tabular}
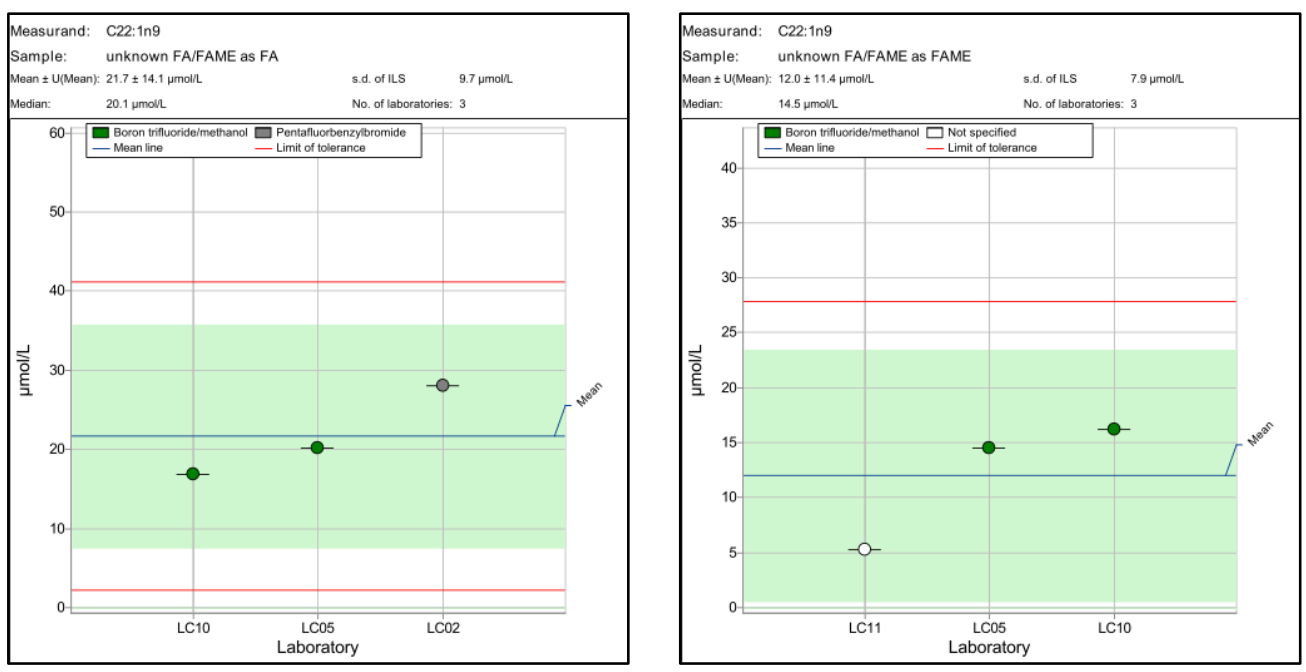
Table B-26: C22:4n6 Docosatetraenoic acid, $\mu \mathrm{mol} / \mathrm{L}$

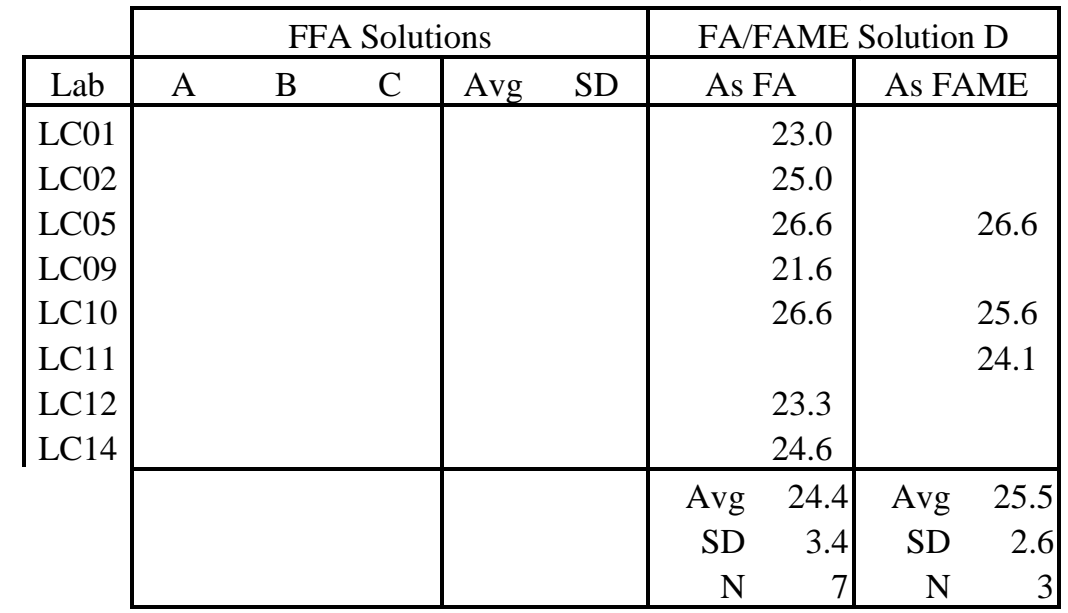
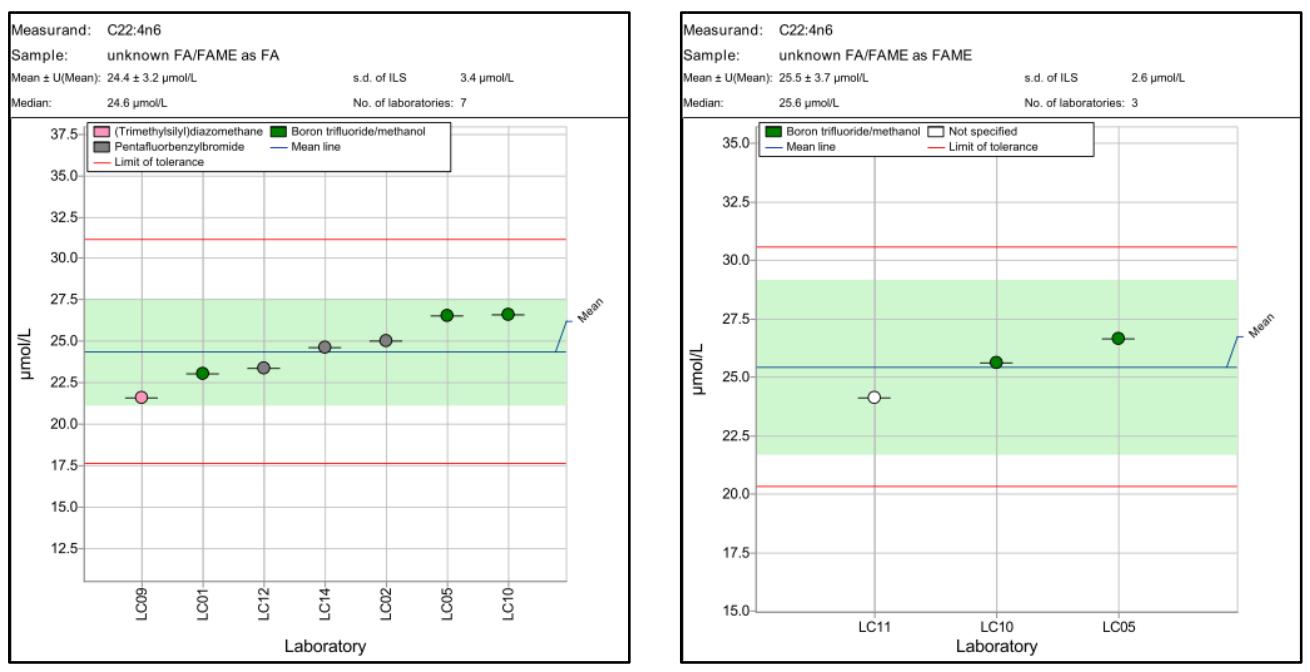
Table B-27: C22:5n3 Docosapentaenoic acid, $\mu \mathrm{mol} / \mathrm{L}$

\begin{tabular}{|c|c|c|c|c|c|c|c|c|c|}
\hline & \multicolumn{5}{|c|}{ FFA Solutions } & \multicolumn{4}{|c|}{ FA/FAME Solution D } \\
\hline Lab & A & B & $\mathrm{C}$ & Avg & SD & As & & As $\mathrm{F}$ & $\mathrm{ME}$ \\
\hline LC01 & 33.4 & 33.6 & 38.9 & 35.3 & 3.1 & & 53.5 & \multirow{5}{*}{\multicolumn{2}{|c|}{57.0}} \\
\hline LC02 & 29.0 & 30.0 & 30.0 & 29.7 & 0.58 & & 44.0 & & \\
\hline LC04 & 39.5 & 40.4 & 41.4 & 40.5 & 0.95 & & & & \\
\hline LC05 & 38.7 & 38.6 & 38.5 & 38.6 & 0.08 & & 56.1 & & \\
\hline LC09 & 42.1 & 35.4 & 35.3 & 37.6 & 3.9 & & 54.8 & & \\
\hline LC10 & 36.2 & 35.1 & 37.3 & 36.2 & 1.1 & & 57.8 & \multirow{4}{*}{\multicolumn{2}{|c|}{$\begin{array}{l}54.8 \\
48.1\end{array}$}} \\
\hline LC11 & & & & & & & & & \\
\hline LC12 & 33.4 & 32.9 & 33.7 & 33.3 & 0.40 & & 49.2 & & \\
\hline \multirow[t]{4}{*}{ LC14 } & 37.9 & 37.4 & 36.4 & 37.3 & 0.8 & & 52.1 & & \\
\hline & & & & Avg & 36.0 & Avg & 52.5 & Avg & \\
\hline & & & & SD & 4.7 & SD & & SD & 7.4 \\
\hline & & & & $\mathrm{N}$ & 8 & $\mathrm{~N}$ & 7 & $\mathrm{~N}$ & \\
\hline
\end{tabular}

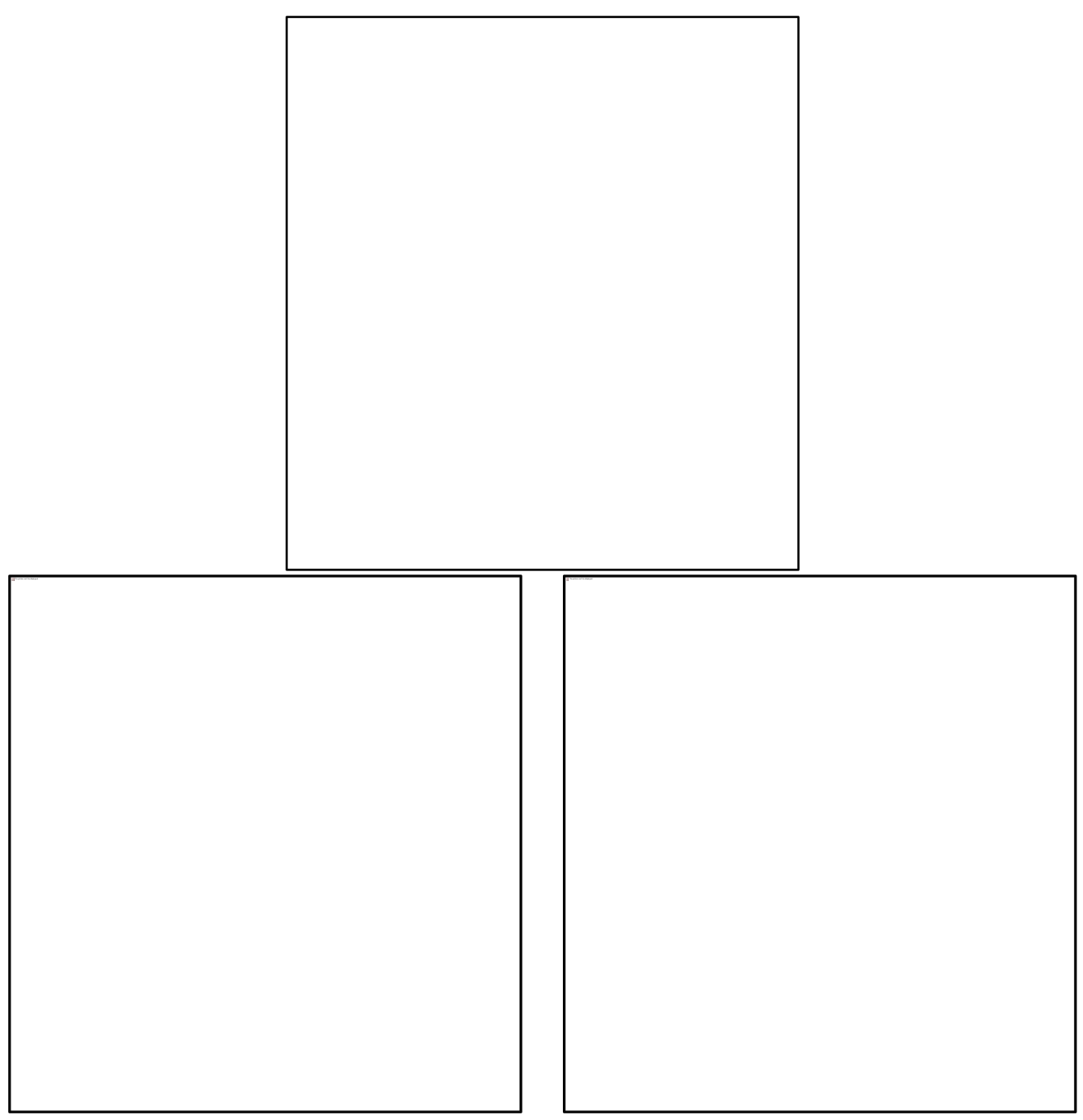


Table B-28: C22:5n6 Docosapentaenoic acid, $\mu \mathrm{mol} / \mathrm{L}$

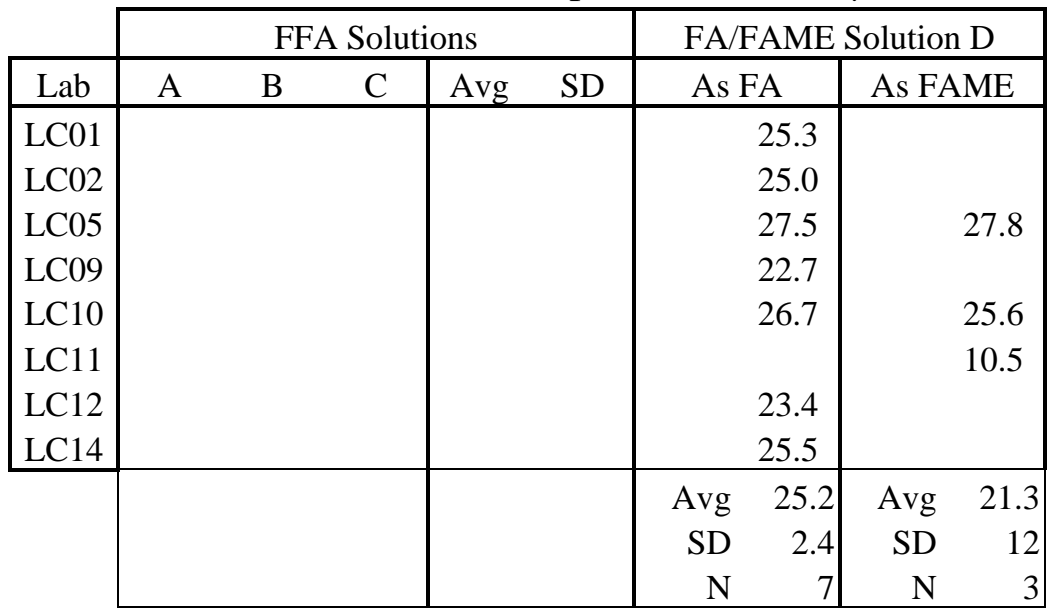
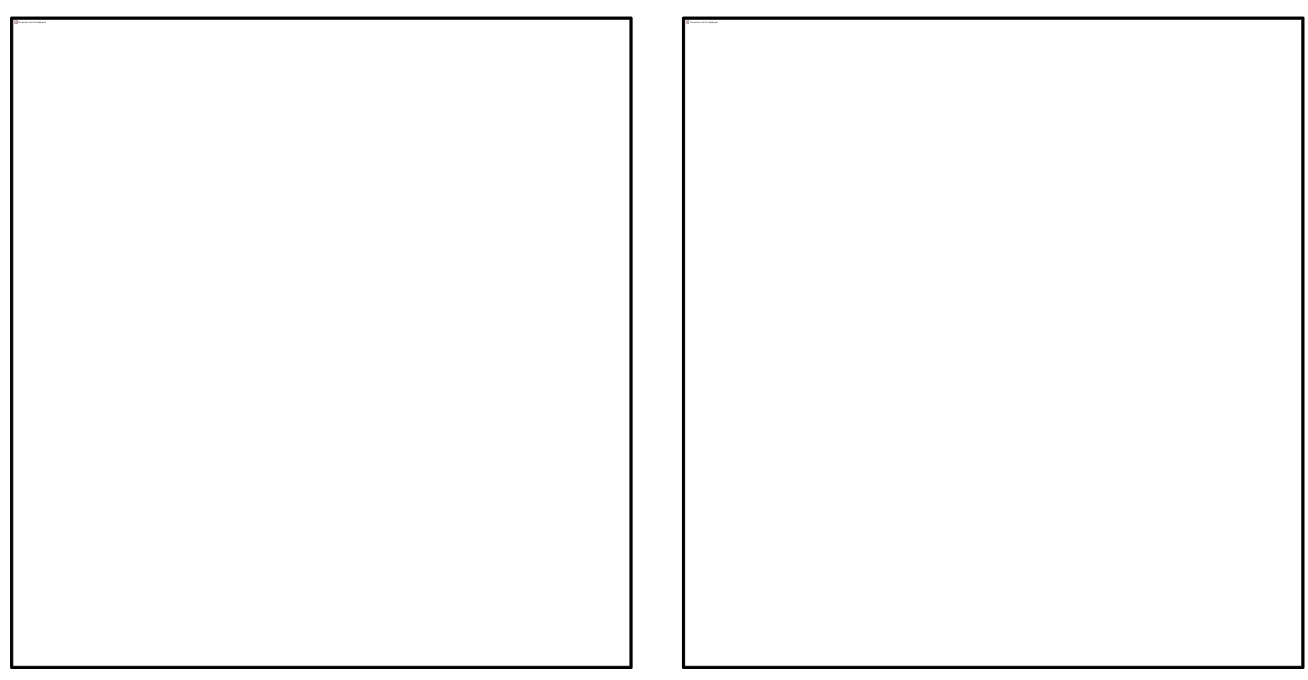
Table B-29: C22:6n3 Docosahexaenoic acid, $\mu \mathrm{mol} / \mathrm{L}$

\begin{tabular}{|c|c|c|c|c|c|c|c|c|}
\hline & \multicolumn{5}{|c|}{ FFA Solutions } & \multicolumn{3}{|c|}{ FA/FAME Solution D } \\
\hline Lab & A & B & $\mathrm{C}$ & Avg & SD & As FA & As $\mathrm{F}$ & $\mathrm{ME}$ \\
\hline LC01 & 92.7 & 92.6 & 91.3 & 92.2 & 0.77 & 260 & & \\
\hline LC02 & 87.0 & 85.0 & 87.0 & 86.3 & 1.15 & 240 & & \\
\hline LC04 & 82.5 & 84.0 & 80.0 & 82.2 & 2.0 & & & \\
\hline LC05 & 100 & 100 & 98.9 & 99.7 & 0.69 & 278 & & 281 \\
\hline LC07 & 116 & 116 & 119 & 117 & 1.7 & 304 & & 341 \\
\hline LC09 & 125 & 104 & 102 & 110 & 12 & 320 & & \\
\hline LC10 & 92.8 & 91.8 & 90.7 & 91.8 & 1.1 & 314 & & 301 \\
\hline LC11 & & & & & & & & 319 \\
\hline LC12 & 79.9 & 79.8 & 80.0 & 79.9 & 0.10 & 238 & & \\
\hline & & & & Avg & 94.9 & Avg & Avg & 310 \\
\hline & & & & SD & 16 & SD & SD & 45 \\
\hline & & & & $\mathrm{N}$ & 8 & $\mathrm{~N}$ & $\mathrm{~N}$ & 4 \\
\hline
\end{tabular}
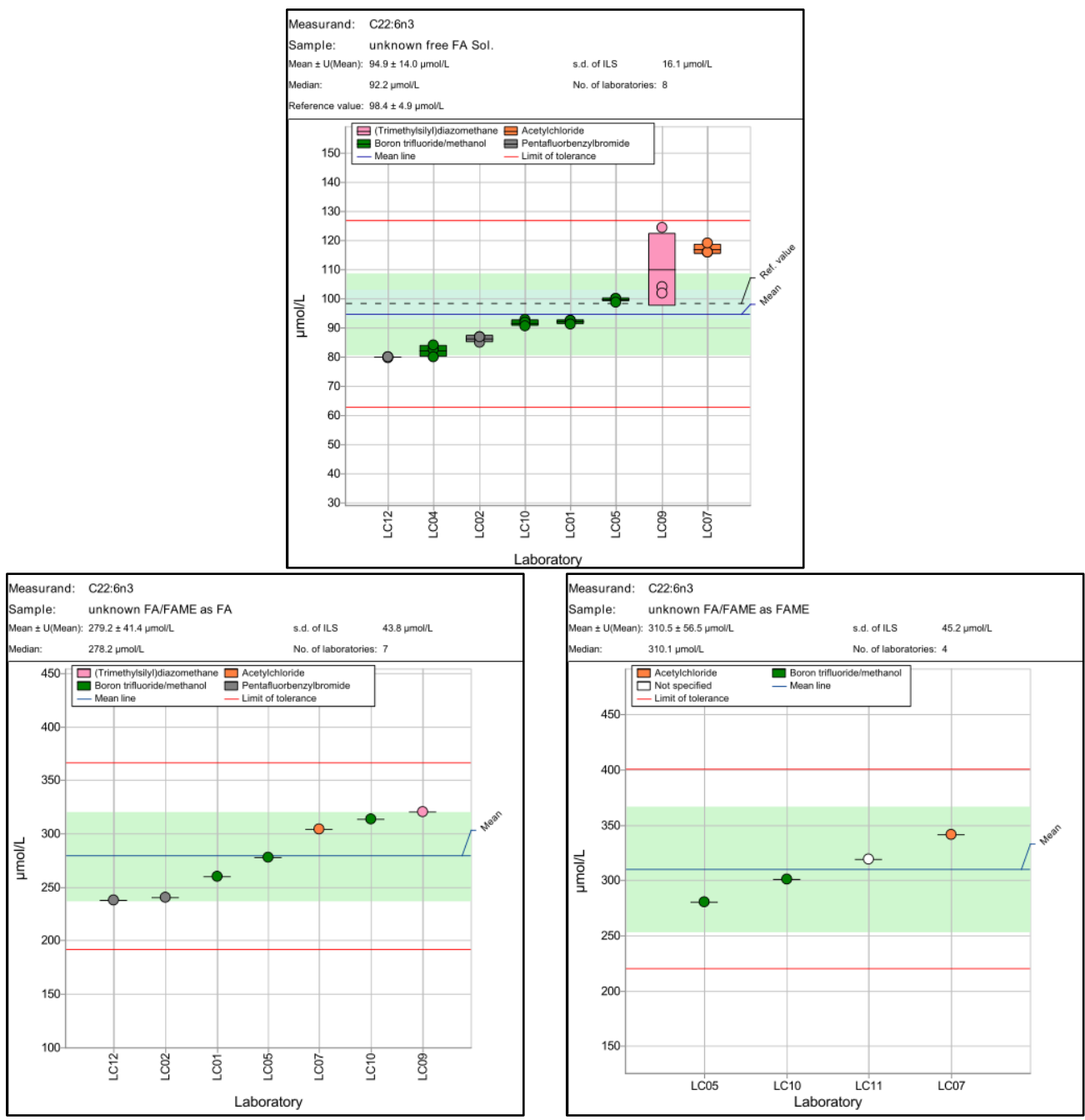
Table B-30: C24:0 Lignoceric acid, $\mu \mathrm{mol} / \mathrm{L}$

\begin{tabular}{|c|c|c|c|c|c|c|c|c|}
\hline & \multicolumn{4}{|c|}{ FFA Solutions } & \multicolumn{4}{|c|}{ FA/FAME Solution D } \\
\hline Lab & A & B & C & Avg S & \multicolumn{2}{|c|}{ As FA } & \multicolumn{2}{|c|}{ As FAME } \\
\hline LC01 & & & & & & 56.9 & & \\
\hline LC02 & & & & & & 53.6 & & \\
\hline LC05 & & & & & & 54.5 & & 55.5 \\
\hline LC11 & & & & & & & & 24.7 \\
\hline LC12 & & & & & & 49.9 & & \\
\hline \multirow[t]{4}{*}{ LC14 } & & & & & & 49.8 & & \\
\hline & & & & & Avg & 52.9 & Avg & 40.1 \\
\hline & & & & & $\mathrm{SD}$ & 5.5 & SD & 34 \\
\hline & & & & & $\mathrm{N}$ & 5 & $\mathrm{~N}$ & 2 \\
\hline
\end{tabular}
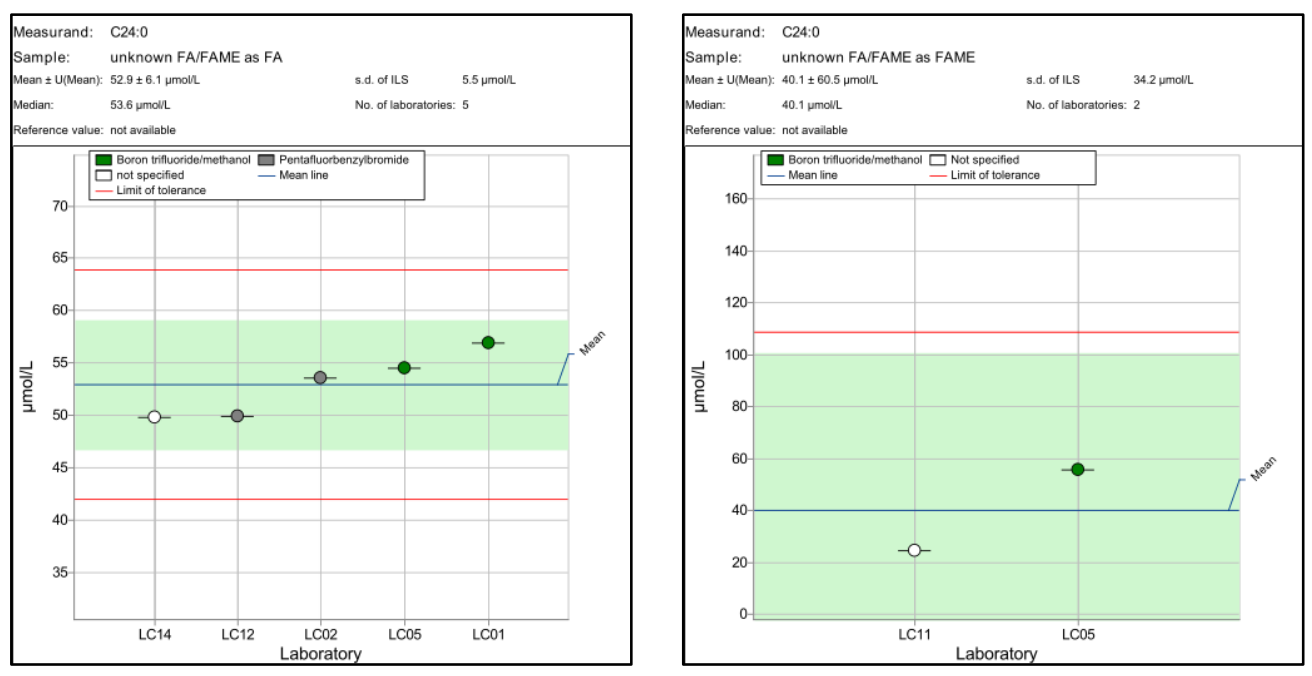
Table B-31: C24:1n9 Nervonic acid, $\mu \mathrm{mol} / \mathrm{L}$

\begin{tabular}{|c|c|c|c|c|c|c|c|c|c|}
\hline & \multicolumn{5}{|c|}{ FFA Solutions } & \multicolumn{4}{|c|}{ FA/FAME Solution D } \\
\hline Lab & A & B & C & Avg & SD & As & & As $\mathrm{F}$ & $\mathrm{ME}$ \\
\hline LC01 & & & & & & & 51.6 & & \\
\hline LC02 & & & & & & & 50.0 & & \\
\hline LC05 & & & & & & & 56.0 & & 56.6 \\
\hline LC07 & & & & & & & 52.0 & & 52.8 \\
\hline LC09 & & & & & & & 46.2 & & \\
\hline LC10 & & & & & & & 41.2 & & 39.7 \\
\hline LC11 & & & & & & & & & 24.5 \\
\hline LC12 & & & & & & & 47.1 & & \\
\hline LC14 & & & & & & & 50.8 & & \\
\hline & & & & & & Avg & 49.4 & Avg & \\
\hline & & & & & & SD & & SD & 29 \\
\hline & & & & & & $\mathrm{N}$ & 8 & $\mathrm{~N}$ & \\
\hline
\end{tabular}
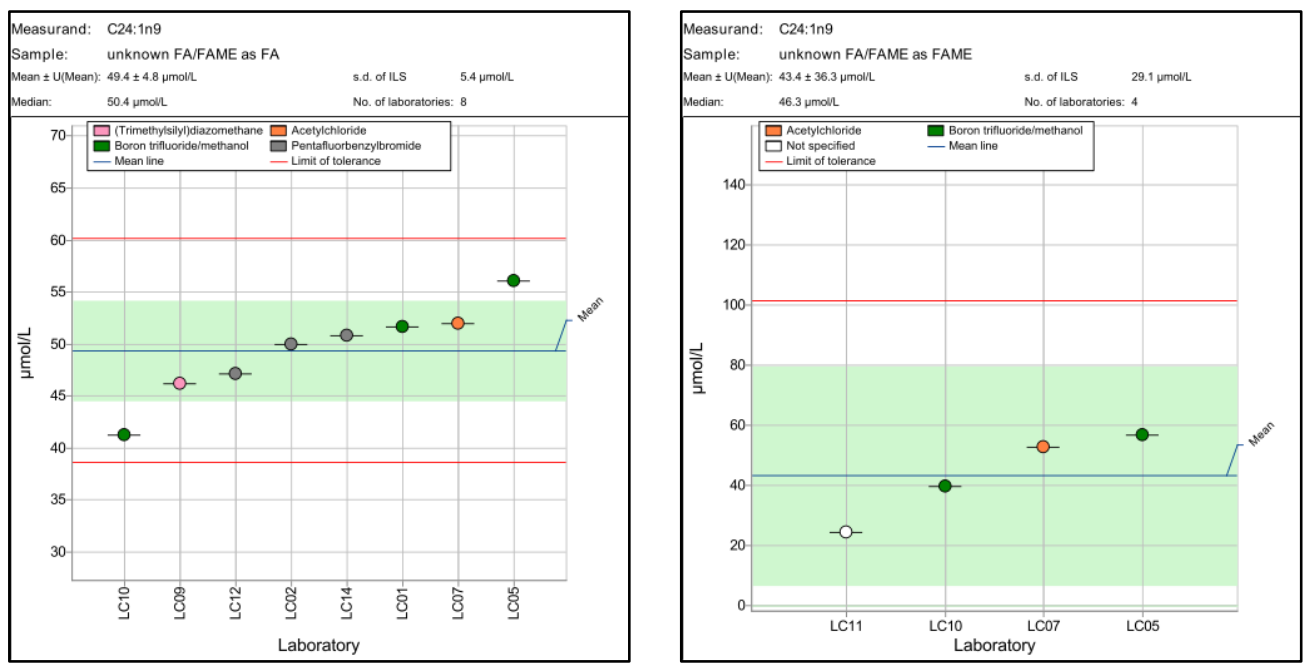


\section{Appendix C: Free Glycerol and Total Glycerides in 2017 Unknown Serum}

Avg

Lab

$\mathrm{N}$

Mean

Acronyms Used in Tables

$\mathrm{SD}$

Participant code

Number of quantitative results

Standard deviation

Table C-32: Free Glycerol, mmol/L

\begin{tabular}{|c|c|ccc|cc|}
\cline { 2 - 7 } \multicolumn{1}{c|}{} & & \multicolumn{4}{c|}{ 2017 Unknown } \\
\hline Lab & SRM 1950 & A & B & C & Avg & SD \\
\hline LC13 & 0.0538 & 0.0018 & 0.0016 & 0.0020 & 0.00183 & 0.000 \\
\hline
\end{tabular}

Table C-2: Total Glycerides (as triolein), mmol/L

\begin{tabular}{|c|c|c|c|c|c|c|}
\hline & & \multicolumn{5}{|c|}{2017 Unknown } \\
\hline Lab & SRM 1950 & A & B & $\mathrm{C}$ & Avg & SD \\
\hline NIST & $1.12 \pm 0.02$ & & & & & \\
\hline LC03 & 1.11 & 1.53 & 1.53 & 1.53 & 1.53 & 0.0035 \\
\hline LC13 & 1.10 & 1.54 & 1.55 & 1.55 & 1.54 & 0.0031 \\
\hline & Avg & & & & Avg & 1.54 \\
\hline & 0.014 & & & & SD & 0.03 \\
\hline & $\mathrm{N}$ & & & & $\mathrm{N}$ & 2 \\
\hline
\end{tabular}
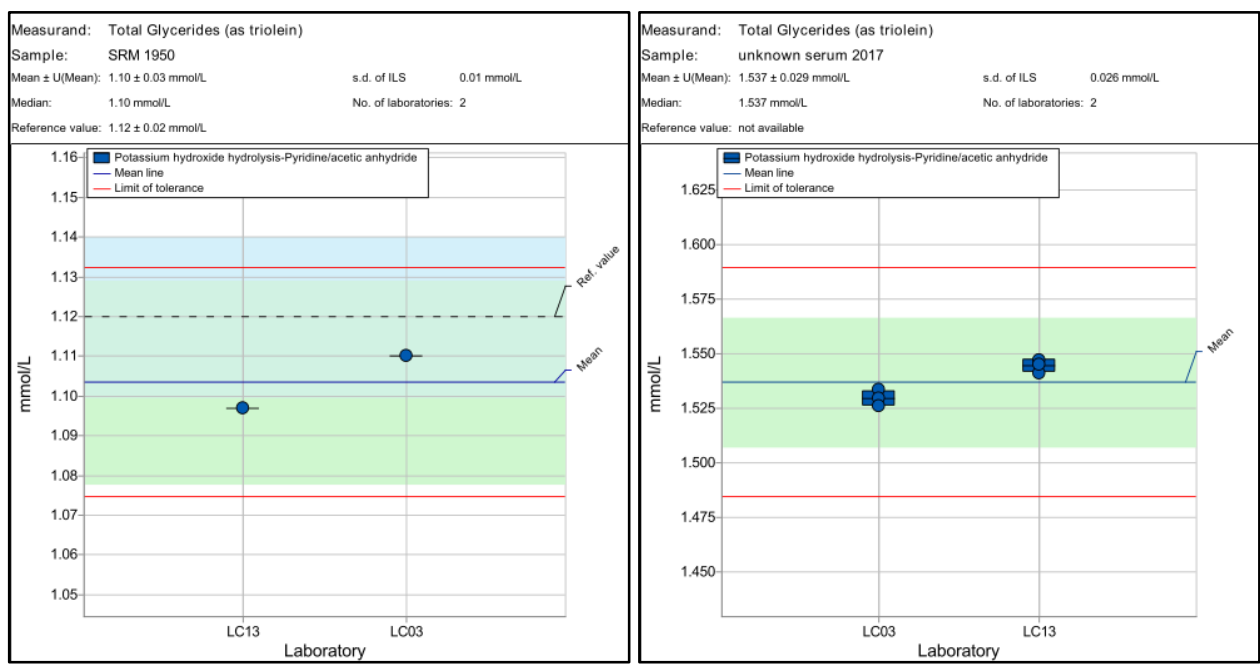


\section{Appendix D: Method Descriptions}

\section{List of Tables}

Table D-1: Method Description for LC01- Total FA Method......................................................... 89

Table D-2: Method Description for LC01-Free FA Method ............................................................. 90

Table D-3: Method Description for LC01-Free FA/FAME in Solutions Method........................... 91

Table D-4: Method Description for LC02-Total FA Method ................................................... 92

Table D-5: Method Description for LC02-Free FA/FAME in Solutions Method.......................... 93

Table D-6: Method Descriptions for LC03-Total Glycerides Method ........................................... 94

Table D-7: Method Descriptions for LC04-Total FA Method .......................................................... 95

Table D-8: Method Descriptions for LC04-Free FA/FAME in Solutions Method ........................ 96

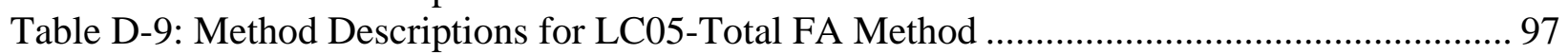

Table D-10: Method Descriptions for LC05-Free FA Method...................................................... 98

Table D-11: Method Descriptions for LC05-Free FA/FAME in Solutions Method ...................... 99

Table D-12: Method Descriptions for LC06-Free FA Method.................................................... 100

Table D-13: Method Descriptions for LC06-Free FA/FAME in Solutions Method ..................... 101

Table D-14: Method Descriptions for LC07: Total FA Method..................................................... 102

Table D-15: Method Descriptions for LC07: Free FA/FAME in Solutions Method ................... 103

Table D-16: Method Descriptions for LC08-Total FA Method ...................................................... 104

Table D-17: Method Descriptions for LC08-Free FA/FAME in Solutions Method .................... 105

Table D-18: Method Descriptions for LC09-Total FA Method ..................................................... 106

Table D-19: Method Descriptions for LC09-Free FA Method........................................................ 107

Table D-20: Method Descriptions for LC09-Free FA/FAME in Solutions Method ..................... 108

Table D-21: Method Descriptions for LC10-Total FA Method ...................................................... 109

Table D-22: Method Descriptions for LC10-Free FA/FAME in Solutions Method ..................... 110

Table D-23: Method Descriptions for LC11-Total FA Method ..................................................... 111

Table D-24: Method Descriptions for LC11-Free FA/FAME in Solutions Method .................... 112

Table D-25: Method Descriptions for LC12-Total FA Method ............................................... 113

Table D-26: Method Description for LC12-Free FA/FAME in Solutions. .................................. 114

Table D-27: Method Descriptions for LC13-Free Glycerol Method............................................. 115

Table D-28: Method Descriptions for LC13-Total Glycerides Method ........................................ 116

Table D-29: Method Descriptions for LC14-Total FA Method .................................................... 117

Table D-30: Method Descriptions for LC14-Free FA/FAME in Solutions Method .................... 118 
Table D-1: Method Description for LC01- Total FA Method

\begin{tabular}{|c|c|c|c|c|c|c|}
\hline & \multicolumn{6}{|c|}{ DESCRIPTION OF PROCEDURES USED FOR TOTAL (CONJUGATED AND FREE) FATTY ACIDS (if evaluated): } \\
\hline \multirow{3}{*}{ 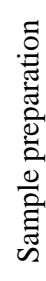 } & Volume of sample extracted (mL): & & A & B & C & $\mathrm{D}$ \\
\hline & \multirow{2}{*}{ Weights of samples extracted (g): } & Unknown Serum 2017 Samples & & & & \\
\hline & & SRM 1950, control & & & & \\
\hline \multirow{7}{*}{ 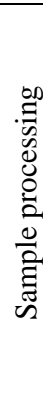 } & Hydrolysis method & \multicolumn{5}{|l|}{$\begin{array}{l}14 \% \text { BF3 in Methanol/Toluene/Methanol } \\
\text { heat 100C } 45 \text { min }\end{array}$} \\
\hline & Extraction method & \multicolumn{5}{|l|}{ LLE } \\
\hline & Extraction solvent & \multicolumn{5}{|l|}{ Hexane } \\
\hline & Extraction time & \multicolumn{5}{|l|}{60 seconds } \\
\hline & Extraction - other details & \multicolumn{5}{|l|}{ add water too } \\
\hline & \multicolumn{6}{|l|}{ Sample extract cleanup method } \\
\hline & Derivatization reagent & \multicolumn{5}{|l|}{$14 \% \mathrm{BF} 3$ in $\mathrm{MeOH}$} \\
\hline \multirow{6}{*}{ 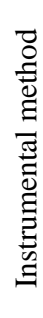 } & Analytical instrument & \multicolumn{5}{|l|}{ GC } \\
\hline & Column phase & \multicolumn{5}{|c|}{ Non-bonded; poly(biscyanopropyl siloxane) (SP-2560) } \\
\hline & Column length, $\mathrm{m}$ & \multicolumn{5}{|c|}{100.00} \\
\hline & Column i.d., mm & \multicolumn{5}{|l|}{$0 . .25$} \\
\hline & Column film thickness, $\mu \mathrm{m}$ & \multicolumn{5}{|l|}{0.20} \\
\hline & Injection method (split, splitless, etc.) & \multicolumn{5}{|l|}{ Split } \\
\hline & Quant Method: ES = external standards $(\mathrm{Y} / \mathrm{N})$ & \multicolumn{5}{|l|}{ Yes } \\
\hline & Number of ES used & \multicolumn{5}{|l|}{3 point standard curve } \\
\hline$\approx$ & Quant Method: IS = internal standards (Y/N) & \multicolumn{5}{|l|}{ Yes } \\
\hline 䔫 & Number of IS used & \multicolumn{5}{|l|}{1.00} \\
\hline च & IS used & \multicolumn{5}{|l|}{ C23:0 TG } \\
\hline $\bar{\sigma}$ & IS added PRIOR to extraction of sample (Y/N) & \multicolumn{5}{|l|}{ Yes } \\
\hline & Calibration model (linear, quad) & \multicolumn{5}{|l|}{ Linear } \\
\hline & Calibration range & \multicolumn{5}{|l|}{ different for each FA } \\
\hline$\dot{\mathscr{m}}$ & \multicolumn{6}{|l|}{ Additional information, method reference } \\
\hline
\end{tabular}


Table D-2: Method Description for LC01-Free FA Method

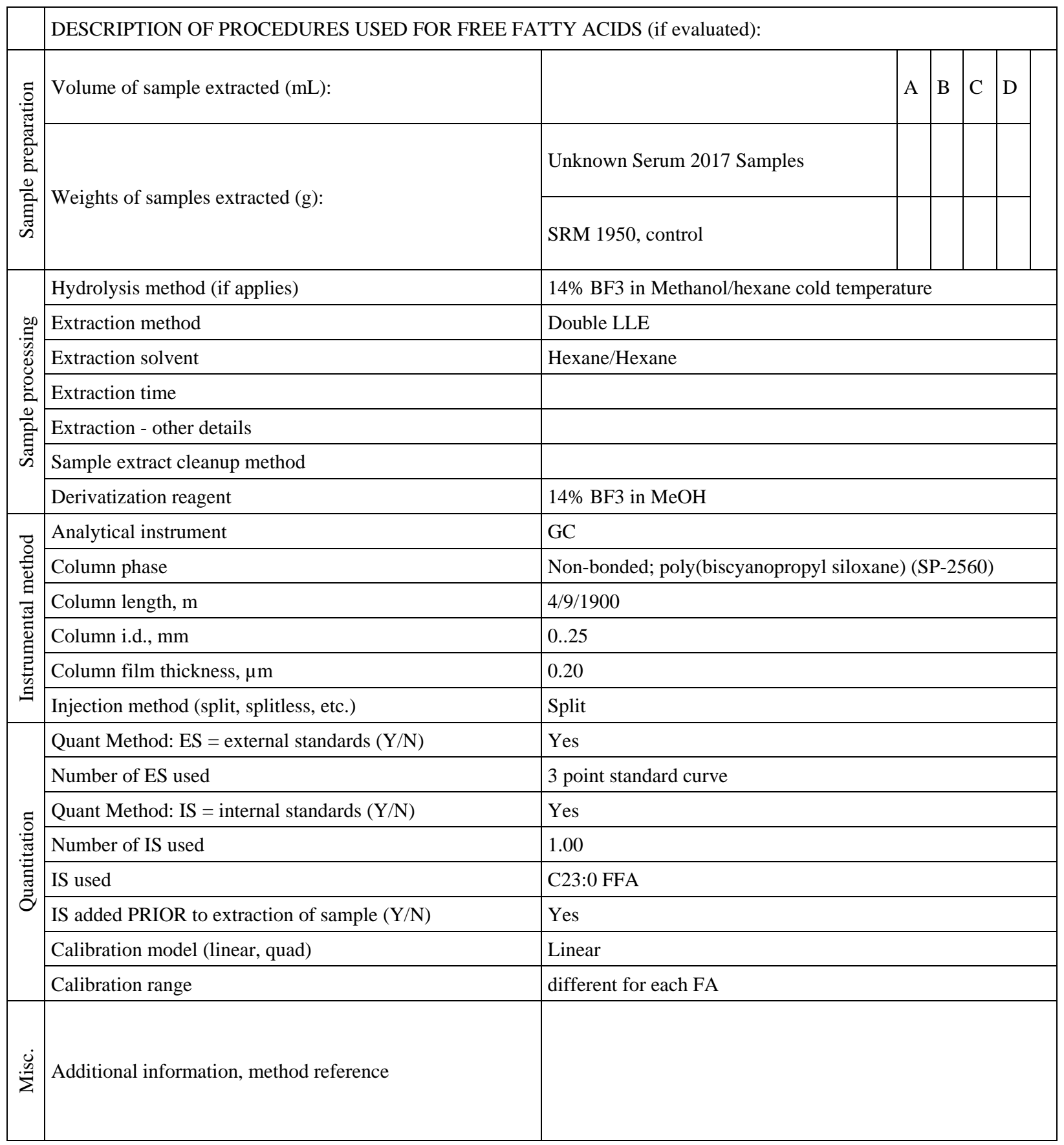


Table D-3: Method Description for LC01-Free FA/FAME in Solutions Method

\begin{tabular}{|c|c|c|c|c|c|c|}
\hline \multirow{3}{*}{ 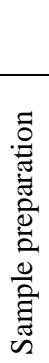 } & \multicolumn{6}{|c|}{ DESCRIPTION OF PROCEDURES USED FOR FREE FATTY ACID/FATTY ACID METHYL ESTER SOLUTIONS } \\
\hline & Volume of sample extracted (mL): & & $\begin{array}{l}\text { Unknown } \\
\text { Free FA Sol. } \\
\text { A }\end{array}$ & $\begin{array}{l}\text { Unknown } \\
\text { Free FA Sol. } \\
\text { B }\end{array}$ & $\begin{array}{l}\text { Unknown } \\
\text { Free FA Sol. } \\
\text { C }\end{array}$ & $\begin{array}{l}\text { Unknown } \\
\text { FAME/FA } \\
\text { Sol. D }\end{array}$ \\
\hline & Weights of samples extracted (g): & Solutions & & & & \\
\hline \multirow{7}{*}{ 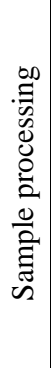 } & Hydrolysis method (if applies) & \multicolumn{5}{|c|}{ 14\% BF3 in Methanol/Toluene/Methanol - heat 100C 45 min } \\
\hline & Extraction method & \multicolumn{5}{|l|}{ LLE } \\
\hline & Extraction solvent & \multicolumn{5}{|l|}{ Hexane } \\
\hline & Extraction time & \multicolumn{5}{|c|}{60 seconds } \\
\hline & Extraction - other details & \multicolumn{5}{|c|}{ add water too } \\
\hline & Sample extract cleanup method & \multicolumn{5}{|c|}{ 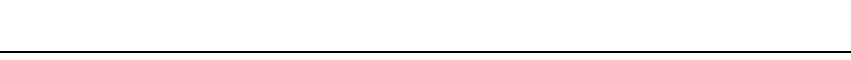 } \\
\hline & Derivatization reagent & \multicolumn{5}{|c|}{$14 \% \mathrm{BF} 3$ in $\mathrm{MeOH}$} \\
\hline \multirow{6}{*}{ 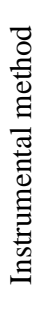 } & Analytical instrument & \multicolumn{5}{|l|}{ GC } \\
\hline & Column phase & \multicolumn{5}{|c|}{ Non-bonded; poly(biscyanopropyl siloxane) (SP-2560) } \\
\hline & Column length, $\mathrm{m}$ & \multicolumn{5}{|l|}{100.00} \\
\hline & Column i.d., mm & \multicolumn{5}{|l|}{$0 . .25$} \\
\hline & Column film thickness, $\mu \mathrm{m}$ & \multicolumn{5}{|l|}{0.20} \\
\hline & Injection method (split, splitless, etc.) & \multicolumn{5}{|l|}{ Split } \\
\hline \multirow{8}{*}{ 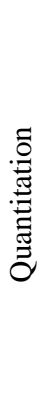 } & Quant Method: ES = external standards (Y/N) & \multicolumn{5}{|l|}{ Yes } \\
\hline & Number of ES used & \multicolumn{5}{|c|}{3 point standard curve } \\
\hline & Quant Method: IS = internal standards (Y/N) & \multicolumn{5}{|l|}{ Yes } \\
\hline & Number of IS used & \multicolumn{5}{|l|}{1.00} \\
\hline & IS used & \multicolumn{5}{|l|}{ C23:0 TG } \\
\hline & IS added PRIOR to extraction of sample (Y/N) & \multicolumn{5}{|l|}{ Yes } \\
\hline & Calibration model (linear, quad) & \multicolumn{5}{|l|}{ Linear } \\
\hline & Calibration range & \multicolumn{5}{|c|}{ different for each FA } \\
\hline 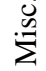 & Additional information, method reference & & & & & \\
\hline
\end{tabular}


Table D-4: Method Description for LC02-Total FA Method

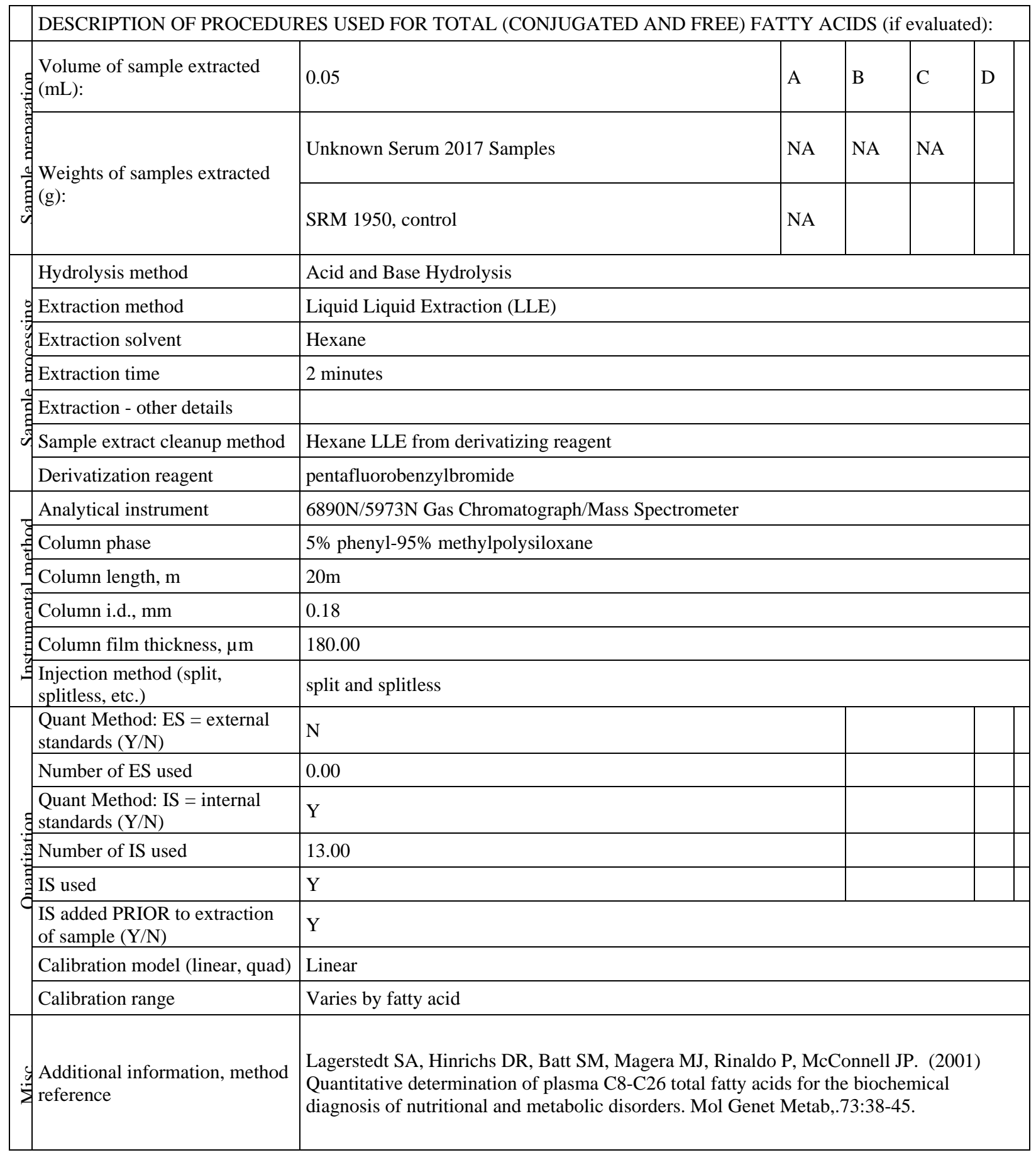


Table D-5: Method Description for LC02-Free FA/FAME in Solutions Method

\begin{tabular}{|c|c|c|c|c|c|c|}
\hline \multirow{4}{*}{ 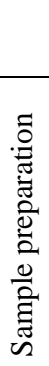 } & \multicolumn{6}{|c|}{ DESCRIPTION OF PROCEDURES USED FOR FREE FATTY ACID/FATTY ACID METHYL ESTER SOLUTIONS } \\
\hline & Volume of sample & & Unknown Free & Unknown Free & Unknown Free & Unknown \\
\hline & extracted (mL): & 0.05 & FA Sol. A & FA Sol. B & FA Sol. C & FAME/FA Sol. D \\
\hline & $\begin{array}{l}\text { Weights of samples } \\
\text { extracted (g): }\end{array}$ & Solutions & NA & NA & NA & NA \\
\hline \multirow{7}{*}{ 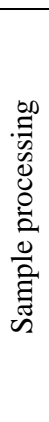 } & $\begin{array}{l}\text { Hydrolysis method (if } \\
\text { applies) }\end{array}$ & \multicolumn{5}{|c|}{ Acid and Base Hydrolysis } \\
\hline & Extraction method & \multicolumn{5}{|c|}{ Liquid Liquid Extraction (LLE) } \\
\hline & Extraction solvent & \multicolumn{5}{|l|}{ Hexane } \\
\hline & Extraction time & \multicolumn{5}{|l|}{2 minutes } \\
\hline & Extraction - other details & & & & & \\
\hline & \begin{tabular}{|l|}
$\begin{array}{l}\text { Sample extract cleanup } \\
\text { method }\end{array}$ \\
\end{tabular} & \multicolumn{5}{|c|}{ Hexane LLE from derivatizing reagent } \\
\hline & Derivatization reagent & \multicolumn{5}{|c|}{ pentafluorobenzylbromide } \\
\hline \multirow{6}{*}{ 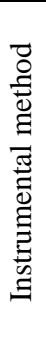 } & Analytical instrument & \multicolumn{5}{|c|}{ 6890N/5973N Gas Chromatograph/Mass Spectrometer } \\
\hline & Column phase & \multicolumn{5}{|c|}{ 5\% phenyl-95\% methylpolysiloxane } \\
\hline & Column length, $\mathrm{m}$ & \multicolumn{5}{|l|}{$20 \mathrm{~m}$} \\
\hline & Column i.d., mm & \multicolumn{5}{|l|}{0.18} \\
\hline & Column film thickness, $\mu \mathrm{m}$ & \multicolumn{5}{|l|}{180.00} \\
\hline & \begin{tabular}{|l|} 
Injection method (split, \\
splitless, etc.)
\end{tabular} & \multicolumn{5}{|c|}{ split and splitless } \\
\hline \multirow{8}{*}{ 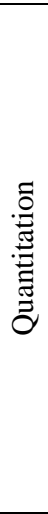 } & $\begin{array}{l}\text { Quant Method: ES = } \\
\text { external standards (Y/N) }\end{array}$ & \multicolumn{2}{|l|}{$\mathrm{N}$} & & & \\
\hline & Number of ES used & \multicolumn{2}{|l|}{0.00} & & & \\
\hline & \begin{tabular}{|l|} 
Quant Method: IS = \\
internal standards (Y/N) \\
\end{tabular} & \multicolumn{2}{|l|}{$\mathrm{Y}$} & & & \\
\hline & Number of IS used & \multicolumn{2}{|l|}{13.00} & & & \\
\hline & IS used & \multicolumn{2}{|l|}{$\mathrm{Y}$} & & & \\
\hline & \begin{tabular}{|l|} 
IS added PRIOR to \\
extraction of sample (Y/N)
\end{tabular} & \multicolumn{5}{|l|}{ Y } \\
\hline & $\begin{array}{l}\text { Calibration model (linear, } \\
\text { quad) }\end{array}$ & \multicolumn{5}{|l|}{ Linear } \\
\hline & Calibration range & \multicolumn{5}{|c|}{ Varies by fatty acid } \\
\hline 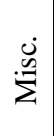 & $\begin{array}{l}\text { Additional information, } \\
\text { method reference }\end{array}$ & \multicolumn{5}{|c|}{$\begin{array}{l}\text { Lagerstedt SA, Hinrichs DR, Batt SM, Magera MJ, Rinaldo P, McConnell JP. (2001) } \\
\text { Quantitative determination of plasma C8-C26 total fatty acids for the biochemical diagnosis } \\
\text { of nutritional and metabolic disorders. Mol Genet Metab,.73:38-45. }\end{array}$} \\
\hline
\end{tabular}


Table D-6: Method Descriptions for LC03-Total Glycerides Method

\begin{tabular}{|c|c|c|c|c|c|c|}
\hline \multirow{4}{*}{ 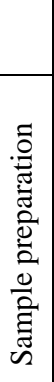 } & \multicolumn{6}{|c|}{ DESCRIPTION OF PROCEDURES USED FOR TOTAL GLYCERIDES (if evaluated): } \\
\hline & Volume of sample extracted (mL): & $0.8 \mathrm{ml}$ & A & B & $\mathrm{C}$ & $\mathrm{D}$ \\
\hline & \multirow{2}{*}{ Weights of samples extracted (g): } & Unknown Serum 2017 Samples & 0.8245 & 0.8251 & 0.8221 & \\
\hline & & SRM 1950, control & 0.8219 & & & \\
\hline \multirow{7}{*}{ 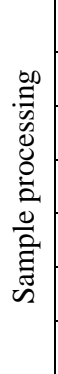 } & Hydrolysis method (if applies) & \multicolumn{5}{|l|}{ Ethanol/KOH solution for $1 \mathrm{~h}, 70 \mathrm{C}$} \\
\hline & Extraction method & \multicolumn{5}{|l|}{ liquid-liquid } \\
\hline & Extraction solvent & \multicolumn{5}{|l|}{ First extraction: ethyl acetate/water } \\
\hline & Extraction time & \multicolumn{5}{|l|}{$15 \mathrm{~min}$} \\
\hline & Extraction - other details & & & & & \\
\hline & Sample extract cleanup method & \multicolumn{5}{|c|}{ Followed by: $2 \mathrm{x}$ sodium bicarbonate and $1 \mathrm{x}$ di water clean up steps } \\
\hline & Derivatization reagent & \multicolumn{5}{|l|}{ acetic anhydride in pyridine } \\
\hline \multirow{6}{*}{ 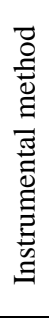 } & Analytical instrument & \multicolumn{5}{|l|}{ GC-MS } \\
\hline & Column phase & \multicolumn{5}{|l|}{ Zebron ZB-50 } \\
\hline & Column length, m & \multicolumn{5}{|l|}{30} \\
\hline & Column i.d., mm & \multicolumn{5}{|l|}{0.25} \\
\hline & Column film thickness, $\mu \mathrm{m}$ & \multicolumn{5}{|l|}{0.25} \\
\hline & Injection method (split, splitless, etc.) & \multicolumn{5}{|l|}{ splitless } \\
\hline & Quant Method: ES = external standards $(\mathrm{Y} / \mathrm{N})$ & \multicolumn{5}{|l|}{$\mathrm{N}$} \\
\hline & Number of ES used & \multicolumn{5}{|l|}{ NA } \\
\hline & Quant Method: IS = internal standards (Y/N) & \multicolumn{5}{|l|}{$\mathrm{Y}$} \\
\hline : & Number of IS used & \multicolumn{5}{|l|}{1} \\
\hline 节 & IS used & \multicolumn{5}{|l|}{ [13C3]-glycerol } \\
\hline$\vec{\sigma}$ & IS added PRIOR to extraction of sample (Y/N) & \multicolumn{5}{|l|}{$\mathrm{Y}$} \\
\hline & Calibration model (linear, quad) & \multicolumn{5}{|l|}{ linear } \\
\hline & Calibration range & \multicolumn{5}{|c|}{$\begin{array}{l}0.056-1.129 \mathrm{mmol} / \mathrm{L} \text { (samples are diluted } \mathrm{x} 3 \text {; therefore the actual } \\
\text { measurement range is } 0.169-3.388 \mathrm{mmol} / \mathrm{L} \text { ) }\end{array}$} \\
\hline 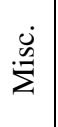 & Additional information, method reference & \multicolumn{5}{|c|}{$\begin{array}{l}\text { Reference Measurement Procedure for Total Glycerides by Isotope } \\
\text { Dilution GC-MS, (JCTLM C12RMP5) } \\
\text { Edwards S. et al., Clinical Chemistry, 2012, 58(4), 768-776 }\end{array}$} \\
\hline
\end{tabular}


Table D-7: Method Descriptions for LC04-Total FA Method

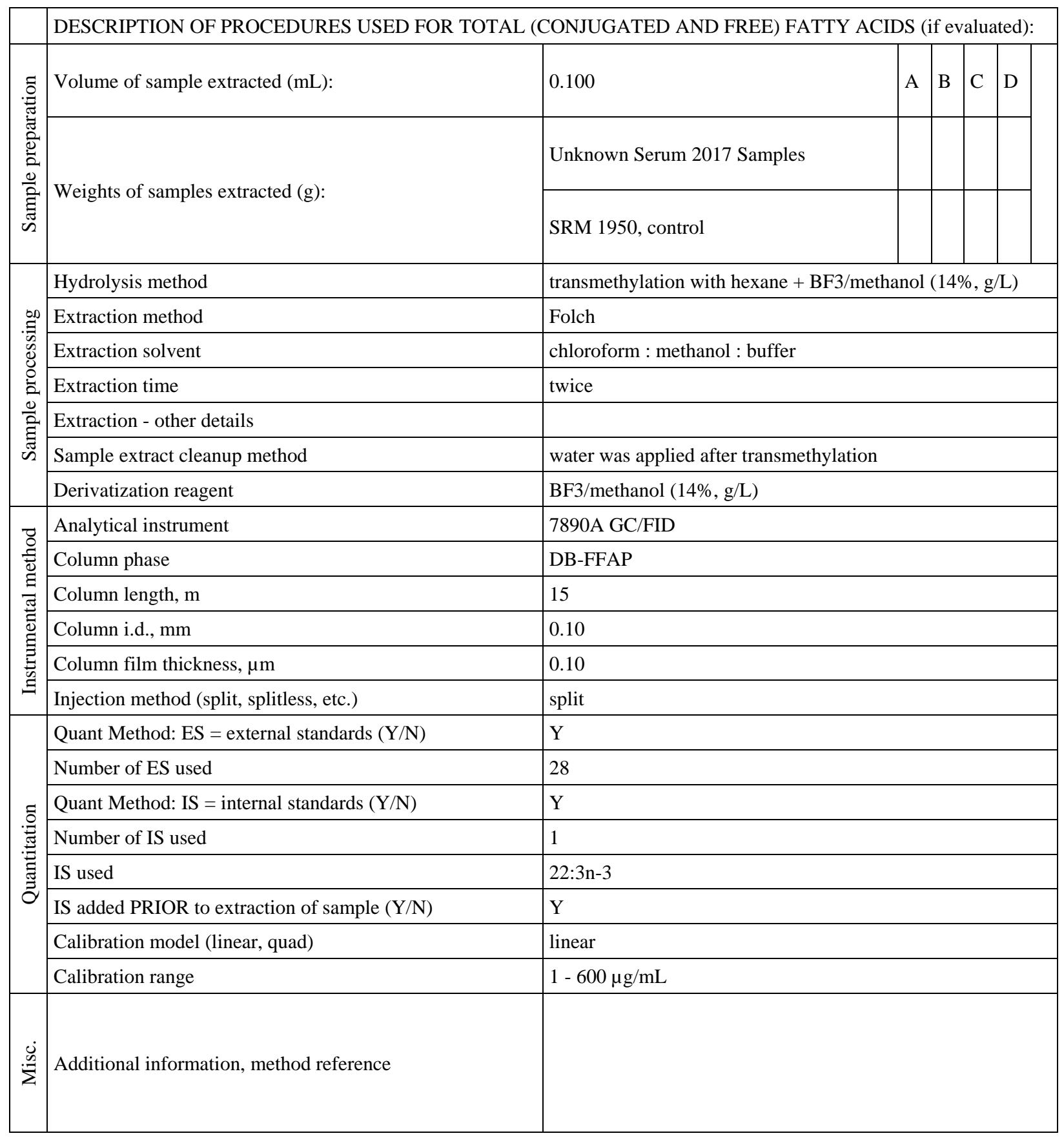


Table D-8: Method Descriptions for LC04-Free FA/FAME in Solutions Method

\begin{tabular}{|c|c|c|c|c|c|c|}
\hline \multirow{3}{*}{ 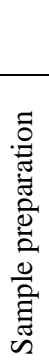 } & \multicolumn{6}{|c|}{ DESCRIPTION OF PROCEDURES USED FOR FREE FATTY ACID/FATTY ACID METHYL ESTER SOLUTIONS } \\
\hline & Volume of sample extracted (mL): & 0.100 & $\begin{array}{l}\text { Unknown } \\
\text { Free FA Sol. } \\
\text { A }\end{array}$ & $\begin{array}{l}\text { Unknown } \\
\text { Free FA Sol. } \\
\text { B }\end{array}$ & $\begin{array}{l}\text { Unknown } \\
\text { Free FA Sol. } \\
\text { C }\end{array}$ & $\begin{array}{l}\text { Unknown } \\
\text { FAME/FA } \\
\text { Sol. D }\end{array}$ \\
\hline & Weights of samples extracted (g): & Solutions & & & & \\
\hline \multirow{7}{*}{ 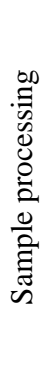 } & Hydrolysis method (if applies) & \multicolumn{5}{|l|}{ N/A } \\
\hline & Extraction method & \multicolumn{5}{|l|}{ N/A } \\
\hline & Extraction solvent & \multicolumn{5}{|l|}{ 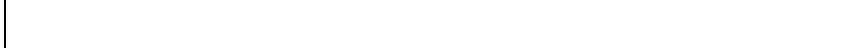 } \\
\hline & Extraction time & \\
\hline & Extraction - other details & \multicolumn{5}{|c|}{ 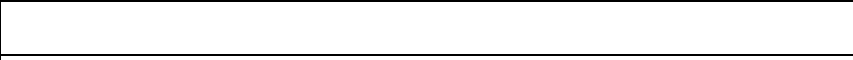 } \\
\hline & Sample extract cleanup method & \multicolumn{5}{|c|}{ 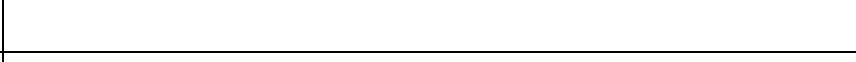 } \\
\hline & Derivatization reagent & \multicolumn{5}{|c|}{ BF3/methanol (14\%, g/L) } \\
\hline \multirow{6}{*}{ 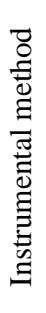 } & Analytical instrument & \multicolumn{5}{|c|}{ same as above } \\
\hline & Column phase & \multicolumn{5}{|c|}{ 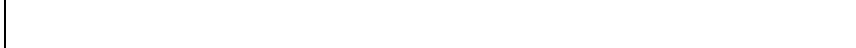 } \\
\hline & Column length, $\mathrm{m}$ & \\
\hline & Column i.d., mm & \\
\hline & Column film thickness, $\mu \mathrm{m}$ & \\
\hline & Injection method (split, splitless, etc.) & \multicolumn{5}{|c|}{ 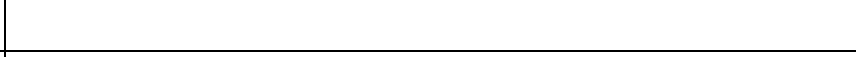 } \\
\hline \multirow{8}{*}{ 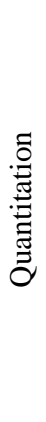 } & Quant Method: ES = external standards (Y/N) & \multicolumn{5}{|c|}{ same as above } \\
\hline & Number of ES used & & & & & \\
\hline & Quant Method: IS = internal standards (Y/N) & & & & & \\
\hline & Number of IS used & \\
\hline & IS used & \\
\hline & IS added PRIOR to extraction of sample (Y/N) & \multicolumn{5}{|l|}{ 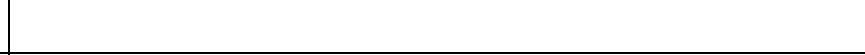 } \\
\hline & Calibration model (linear, quad) & \\
\hline & Calibration range & \\
\hline 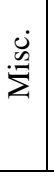 & Additional information, method reference & \\
\hline
\end{tabular}


Table D-9: Method Descriptions for LC05-Total FA Method

\begin{tabular}{|c|c|c|c|c|c|c|}
\hline & \multicolumn{6}{|c|}{ DESCRIPTION OF PROCEDURES USED FOR TOTAL (CONJUGATED AND FREE) FATTY ACIDS (if evaluated): } \\
\hline \multirow{3}{*}{ 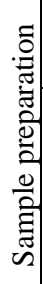 } & Volume of sample extracted (mL): & 0.10 & A & B & $\mathrm{C}$ & $\mathrm{D}$ \\
\hline & \multirow{2}{*}{ Weights of samples extracted (g): } & Unknown Serum 2017 Samples & 0.106 & 0.106 & 0.106 & \\
\hline & & SRM 1950, control & 0.107 & & & \\
\hline \multirow{7}{*}{ 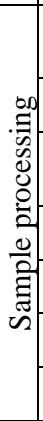 } & Hydrolysis method & \multicolumn{5}{|c|}{$\begin{array}{l}\text { NA, direct transesterification of lipid extracts with methylating } \\
\text { reagents }\end{array}$} \\
\hline & Extraction method & \multicolumn{5}{|l|}{ Modified Folch } \\
\hline & Extraction solvent & \multicolumn{5}{|c|}{$\begin{array}{l}\text { 3mL of 2:1:0.5 chloroform:methanol:0.2M sodium phosphate buffer, } \\
\text { C22:3n-3 free fatty acid internal standard }\end{array}$} \\
\hline & Extraction time & \multicolumn{5}{|c|}{30 minutes } \\
\hline & Extraction - other details & \multicolumn{5}{|l|}{ NA } \\
\hline & Sample extract cleanup method & \multicolumn{5}{|c|}{ FAME cleanup with $1 \mathrm{~mL}$ ddH2O and $1 \mathrm{~mL}$ hexane } \\
\hline & Derivatization reagent & \multicolumn{5}{|c|}{$14 \%$ boron trifluoride in methanol } \\
\hline \multirow{6}{*}{ 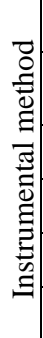 } & Analytical instrument & \multicolumn{5}{|l|}{ Varian 3900 Gas Chromatograph } \\
\hline & Column phase & \multicolumn{5}{|c|}{$\begin{array}{l}\text { DB-FFAP nitroterephthalic acid modified polyethylene glycol } \\
\text { capillary column }\end{array}$} \\
\hline & Column length, $\mathrm{m}$ & \multicolumn{5}{|c|}{15.00} \\
\hline & Column i.d., mm & \multicolumn{5}{|l|}{0.10} \\
\hline & Column film thickness, $\mu \mathrm{m}$ & \multicolumn{5}{|l|}{0.10} \\
\hline & Injection method (split, splitless, etc.) & \multicolumn{5}{|l|}{ Split injection, 1:100 } \\
\hline & Quant Method: ES = external standards (Y/N) & \multicolumn{5}{|l|}{$\mathrm{Y}$} \\
\hline & Number of ES used & \multicolumn{5}{|l|}{39.00} \\
\hline & Quant Method: IS = internal standards (Y/N) & \multicolumn{5}{|l|}{$\mathrm{Y}$} \\
\hline 苛 & Number of IS used & \multicolumn{5}{|l|}{1.00} \\
\hline 节 & IS used & \multicolumn{5}{|l|}{ C 22:3n-3 free fatty acid } \\
\hline$\Xi$ & IS added PRIOR to extraction of sample (Y/N) & \multicolumn{5}{|l|}{$\mathrm{Y}$} \\
\hline & Calibration model (linear, quad) & \multicolumn{5}{|c|}{ Linear } \\
\hline & Calibration range & \multicolumn{5}{|c|}{$\begin{array}{l}\text { 0.0016ug/mL solvent - } 75 \mathrm{ug} / \mathrm{mL} \text { solvent for each fatty acid, using } \\
\text { GLC-462 }\end{array}$} \\
\hline 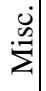 & \multicolumn{6}{|l|}{ Additional information, method reference } \\
\hline
\end{tabular}


Table D-10: Method Descriptions for LC05-Free FA Method

\begin{tabular}{|c|c|c|c|c|c|c|}
\hline \multirow{4}{*}{ 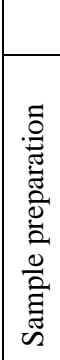 } & \multicolumn{6}{|c|}{ DESCRIPTION OF PROCEDURES USED FOR FREE FATTY ACIDS (if evaluated): } \\
\hline & Volume of sample extracted (mL): & 0.10 & A & B & C & $\mathrm{D}$ \\
\hline & \multirow{2}{*}{ Weights of samples extracted (g): } & Unknown Serum 2017 Samples & 0.107 & 0.106 & 0.107 & \\
\hline & & SRM 1950, control & 0.107 & & & \\
\hline \multirow{7}{*}{ 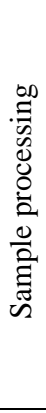 } & Hydrolysis method (if applies) & \multicolumn{5}{|l|}{ NA } \\
\hline & Extraction method & \multicolumn{5}{|c|}{ Modified Folch } \\
\hline & Extraction solvent & \multicolumn{5}{|c|}{$\begin{array}{l}\text { 3mL of 2:1:0.5 chloroform:methanol:0.2M sodium phosphate buffer, } \\
\text { C22:3n-3 free fatty acid internal standard }\end{array}$} \\
\hline & Extraction time & \multicolumn{5}{|l|}{30 minutes } \\
\hline & Extraction - other details & \multicolumn{5}{|l|}{ NA } \\
\hline & Sample extract cleanup method & \multicolumn{5}{|c|}{ FAME cleanup with $1 \mathrm{~mL}$ ddH2O and $1 \mathrm{~mL}$ hexane } \\
\hline & Derivatization reagent & \multicolumn{5}{|c|}{$\begin{array}{l}\text { 14\% boron trifluoride in methanol after FFA separation by thin layer } \\
\text { chromatography and extraction using chloroform/methanol }\end{array}$} \\
\hline \multirow{6}{*}{ 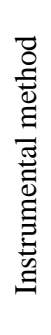 } & Analytical instrument & \multicolumn{5}{|l|}{ Varian 3900 Gas Chromatograph } \\
\hline & Column phase & \multicolumn{5}{|c|}{$\begin{array}{l}\text { DB-FFAP nitroterephthalic acid modified polyethylene glycol capillary } \\
\text { column }\end{array}$} \\
\hline & Column length, m & \multicolumn{5}{|c|}{15.00} \\
\hline & Column i.d., mm & \multicolumn{5}{|l|}{0.10} \\
\hline & Column film thickness, $\mu \mathrm{m}$ & \multicolumn{5}{|l|}{0.10} \\
\hline & Injection method (split, splitless, etc.) & \multicolumn{5}{|l|}{ Split injection, 1:20 } \\
\hline \multirow{8}{*}{ 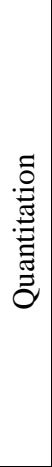 } & Quant Method: ES = external standards $(\mathrm{Y} / \mathrm{N})$ & \multicolumn{5}{|l|}{ Y } \\
\hline & Number of ES used & \multicolumn{5}{|l|}{39.00} \\
\hline & Quant Method: IS = internal standards $(\mathrm{Y} / \mathrm{N})$ & \multicolumn{5}{|l|}{ Y } \\
\hline & Number of IS used & \multicolumn{5}{|l|}{1.00} \\
\hline & IS used & \multicolumn{5}{|l|}{ C 22:3n-3 free fatty acid } \\
\hline & $\begin{array}{l}\text { IS added PRIOR to extraction of sample } \\
\text { (Y/N) }\end{array}$ & \multicolumn{5}{|l|}{ Y } \\
\hline & Calibration model (linear, quad) & \multicolumn{5}{|l|}{ Linear } \\
\hline & Calibration range & \multicolumn{5}{|c|}{$\begin{array}{l}0.0016 \mathrm{ug} / \mathrm{mL} \text { solvent - } 75 \mathrm{ug} / \mathrm{mL} \text { solvent for each fatty acid, using GLC- } \\
462\end{array}$} \\
\hline$\stackrel{\leftrightarrow}{\stackrel{0}{2}}$ & Additional information, method reference & \multicolumn{5}{|c|}{$\begin{array}{l}\text { Thin layer chromatography was performed using a mobile phase consisting } \\
\text { of 60:40:2 heptane:anhydrous diethyl ether:glacial acetic acid, used a } \\
\text { mixture of external reference standards for visualization under UV light, } \\
\text { samples were scraped and lipids were extracted from silica shavings using } \\
\text { 3mL 2:1 chloroform:methanol. Free fatty acid extracts were then } \\
\text { methylated and analyzed by GC. }\end{array}$} \\
\hline
\end{tabular}


Table D-11: Method Descriptions for LC05-Free FA/FAME in Solutions Method

\begin{tabular}{|c|c|c|c|c|c|c|}
\hline \multirow{3}{*}{ 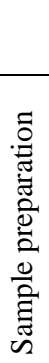 } & \multicolumn{6}{|c|}{ DESCRIPTION OF PROCEDURES USED FOR FREE FATTY ACID/FATTY ACID METHYL ESTER SOLUTIONS: } \\
\hline & Volume of sample extracted (mL): & 0.10 & $\begin{array}{l}\text { Unknown } \\
\text { Free FA Sol. } \\
\text { A }\end{array}$ & $\begin{array}{l}\text { Unknown } \\
\text { Free FA Sol. } \\
\text { B }\end{array}$ & $\begin{array}{l}\text { Unknown } \\
\text { Free FA Sol. } \\
\text { C }\end{array}$ & $\begin{array}{l}\text { Unknown } \\
\text { FAME/FA } \\
\text { Sol. D }\end{array}$ \\
\hline & Weights of samples extracted (g): & Solutions & 100uL & $100 \mathrm{uL}$ & $100 \mathrm{uL}$ & $100 \mathrm{uL}$ \\
\hline \multirow{7}{*}{ 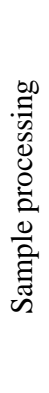 } & Hydrolysis method (if applies) & \multicolumn{5}{|l|}{ NA } \\
\hline & Extraction method & \multicolumn{5}{|c|}{$\begin{array}{l}\text { NA, FFA and FAME solutions were derivatized to generate FAME after } \\
\text { aliquoting samples and evaporating toluene under N2 gas }\end{array}$} \\
\hline & Extraction solvent & \multicolumn{5}{|c|}{ NA } \\
\hline & Extraction time & \multicolumn{5}{|l|}{ NA } \\
\hline & Extraction - other details & \multicolumn{5}{|l|}{ NA } \\
\hline & Sample extract cleanup method & \multicolumn{5}{|c|}{ FAME cleanup with $1 \mathrm{~mL}$ ddH2O and $1 \mathrm{~mL}$ hexane } \\
\hline & Derivatization reagent & \multicolumn{5}{|c|}{$14 \%$ boron trifluoride in methanol } \\
\hline \multirow{6}{*}{ 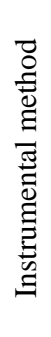 } & Analytical instrument & \multicolumn{5}{|c|}{ Varian 3900 Gas Chromatograph } \\
\hline & Column phase & \multicolumn{5}{|c|}{$\begin{array}{l}\text { DB-FFAP nitroterephthalic acid modified polyethylene glycol capillary } \\
\text { column }\end{array}$} \\
\hline & Column length, $\mathrm{m}$ & \multicolumn{5}{|c|}{15.00} \\
\hline & Column i.d., mm & \multicolumn{5}{|l|}{0.10} \\
\hline & Column film thickness, $\mu \mathrm{m}$ & \multicolumn{5}{|l|}{0.10} \\
\hline & Injection method (split, splitless, etc.) & \multicolumn{5}{|c|}{ Split injection, 1:100 } \\
\hline & $\begin{array}{l}\text { Quant Method: ES = external standards } \\
(\mathrm{Y} / \mathrm{N})\end{array}$ & \multicolumn{5}{|l|}{ Y } \\
\hline & Number of ES used & \multicolumn{5}{|l|}{39.00} \\
\hline ๘ & $\begin{array}{l}\text { Quant Method: IS = internal standards } \\
(\mathrm{Y} / \mathrm{N})\end{array}$ & \multicolumn{5}{|l|}{$\mathrm{Y}$} \\
\hline 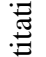 & Number of IS used & \multicolumn{5}{|l|}{1.00} \\
\hline 䒿 & IS used & \multicolumn{5}{|c|}{ C 22:3n-3 free fatty acid } \\
\hline$\sigma$ & $\begin{array}{l}\text { IS added PRIOR to extraction of sample } \\
(\mathrm{Y} / \mathrm{N})\end{array}$ & \multicolumn{5}{|l|}{$\mathrm{Y}$} \\
\hline & Calibration model (linear, quad) & \multicolumn{5}{|l|}{ Linear } \\
\hline & Calibration range & \multicolumn{5}{|c|}{ 0.0016ug/mL solvent - $75 \mathrm{ug} / \mathrm{mL}$ solvent for each fatty acid, using GLC-462 } \\
\hline 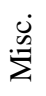 & Additional information, method reference & \multicolumn{5}{|c|}{$\begin{array}{l}\text { Samples were not weighed to determine density because of high volatility of } \\
\text { sample solvent, scale did not stabilize }\end{array}$} \\
\hline
\end{tabular}


Table D-12: Method Descriptions for LC06-Free FA Method

\begin{tabular}{|c|c|c|c|c|c|c|}
\hline & \multicolumn{6}{|c|}{ DESCRIPTION OF PROCEDURES USED FOR FREE FATTY ACIDS (if evaluated): } \\
\hline \multirow{3}{*}{ 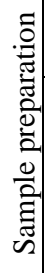 } & Volume of sample extracted (mL): & & A & B & $\mathrm{C}$ & D \\
\hline & & Unknown Serum 2017 Samples & 0.25268 & 0.25571 & 0.25745 & \\
\hline & Weights of samples extracted (g): & SRM 1950, control & 0.25 & & & \\
\hline \multirow{7}{*}{ 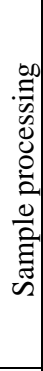 } & Hydrolysis method (if applies) & \multicolumn{5}{|l|}{ None } \\
\hline & Extraction method & \multicolumn{5}{|l|}{ 2, $2 \mathrm{~mL}$, liquid/liquid extractions. } \\
\hline & Extraction solvent & \multicolumn{5}{|l|}{ ethyl acetate } \\
\hline & Extraction time & \multicolumn{5}{|l|}{1 min vortex } \\
\hline & \multicolumn{6}{|l|}{ Extraction - other details } \\
\hline & Sample extract cleanup method & \multicolumn{5}{|l|}{ none } \\
\hline & Derivatization reagent & \multicolumn{5}{|l|}{ none } \\
\hline \multirow{6}{*}{ 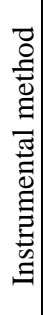 } & Analytical instrument & \multicolumn{5}{|l|}{ Agilent 5975C } \\
\hline & Column phase & \multicolumn{5}{|l|}{ ZB-FFAP } \\
\hline & Column length, m & \multicolumn{5}{|l|}{60} \\
\hline & Column i.d., mm & \multicolumn{5}{|l|}{0.25} \\
\hline & Column film thickness, $\mu \mathrm{m}$ & \multicolumn{5}{|l|}{0.25} \\
\hline & Injection method (split, splitless, etc.) & \multicolumn{5}{|l|}{ on-column } \\
\hline & Quant Method: ES = external standards (Y/N) & \multicolumn{5}{|l|}{$\mathrm{N}$} \\
\hline & Number of ES used & \multicolumn{5}{|l|}{ NA } \\
\hline 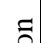 & Quant Method: IS = internal standards (Y/N) & \multicolumn{5}{|l|}{$\mathrm{Y}$} \\
\hline : & Number of IS used & \multicolumn{5}{|l|}{3.00} \\
\hline 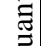 & IS used & \multicolumn{5}{|c|}{ myristic-d27, palmitic-d31, and steric-d35 } \\
\hline 0 & IS added PRIOR to extraction of sample (Y/N) & \multicolumn{5}{|l|}{$\mathrm{Y}$} \\
\hline & Calibration model (linear, quad) & \multicolumn{5}{|l|}{ single point } \\
\hline \multicolumn{7}{|c|}{ Calibration range } \\
\hline$\dot{\mathscr{n}}$ & Additional information, method reference & & & & & \\
\hline
\end{tabular}


Table D-13: Method Descriptions for LC06-Free FA/FAME in Solutions Method

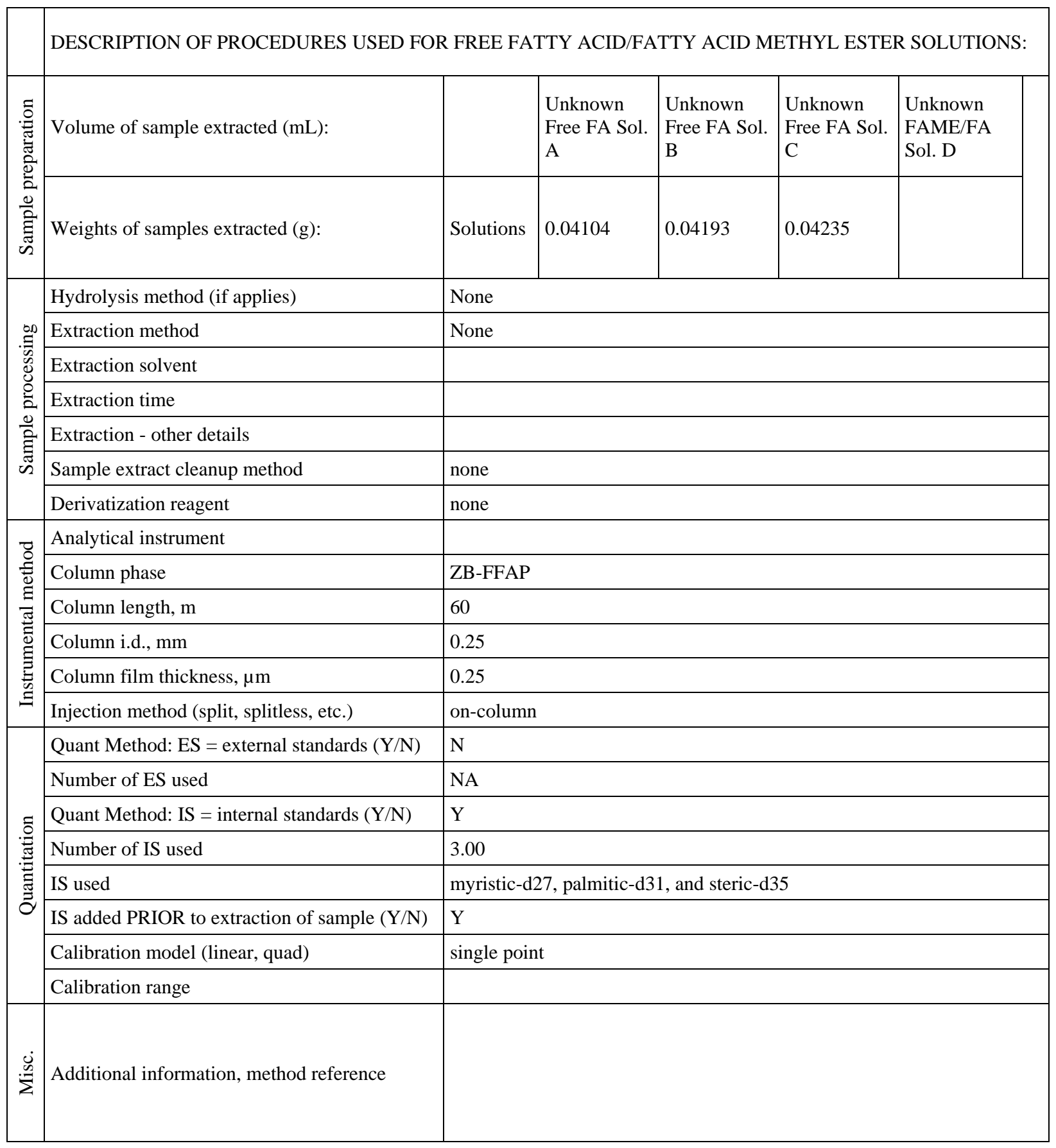


Table D-14: Method Descriptions for LC07: Total FA Method

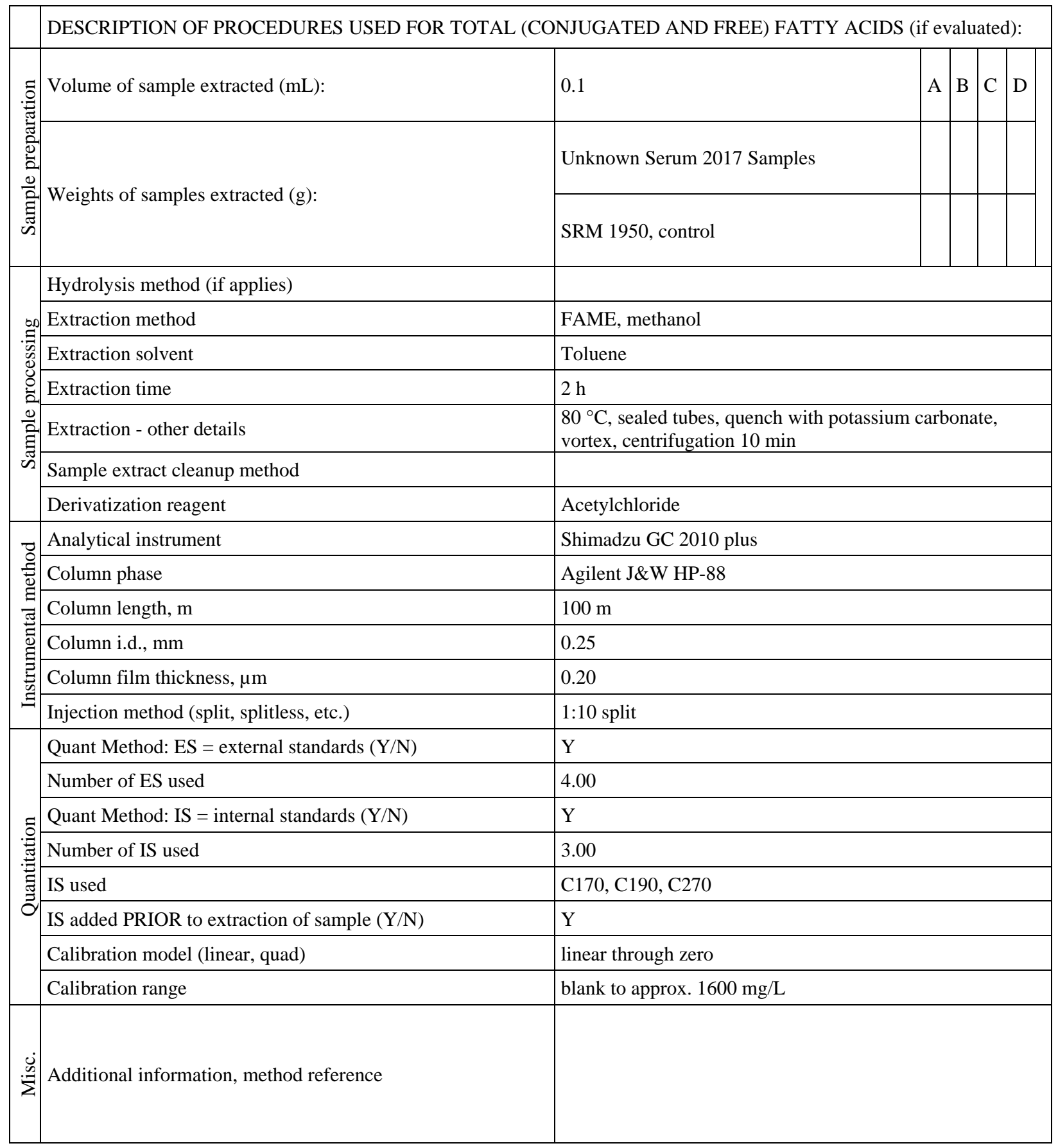


Table D-15: Method Descriptions for LC07: Free FA/FAME in Solutions Method

\begin{tabular}{|c|c|c|c|c|c|c|}
\hline \multirow{3}{*}{ 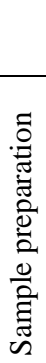 } & \multicolumn{6}{|c|}{ DESCRIPTION OF PROCEDURES USED FOR FREE FATTY ACID/FATTY ACID METHYL ESTER SOLUTIONS: } \\
\hline & Volume of sample extracted (mL): & 0.10 & $\begin{array}{l}\text { Unknown } \\
\text { Free FA Sol. } \\
\text { A }\end{array}$ & $\begin{array}{l}\text { Unknown } \\
\text { Free FA Sol. } \\
\text { B }\end{array}$ & $\begin{array}{l}\text { Unknown } \\
\text { Free FA Sol. } \\
\text { C }\end{array}$ & $\begin{array}{l}\text { Unknown } \\
\text { FAME/FA } \\
\text { Sol. D }\end{array}$ \\
\hline & Weights of samples extracted (g): & Solutions & & & & \\
\hline \multirow{7}{*}{ 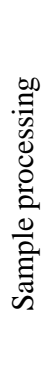 } & Hydrolysis method (if applies) & & & & & \\
\hline & Extraction method & \multicolumn{5}{|c|}{ FAME, methanol } \\
\hline & Extraction solvent & \multicolumn{5}{|l|}{ Toluene } \\
\hline & Extraction time & \multicolumn{5}{|c|}{$2 \mathrm{~h}$} \\
\hline & Extraction - other details & \multicolumn{5}{|c|}{$\begin{array}{l}80^{\circ} \mathrm{C} \text {, sealed tubes, quench with potassium carbonate, vortex, } \\
\text { centrifugation } 10 \mathrm{~min}\end{array}$} \\
\hline & \multicolumn{6}{|l|}{ Sample extract cleanup method } \\
\hline & Derivatization reagent & \multicolumn{5}{|c|}{ Acetylchloride } \\
\hline \multirow{6}{*}{ 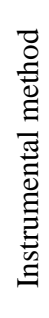 } & Analytical instrument & \multicolumn{5}{|c|}{ Shimadzu GC 2010 plus } \\
\hline & Column phase & \multicolumn{5}{|c|}{ Agilent J\&W HP-88 } \\
\hline & Column length, $\mathrm{m}$ & \multicolumn{5}{|l|}{$100 \mathrm{~m}$} \\
\hline & Column i.d., mm & \multicolumn{5}{|l|}{0.25} \\
\hline & Column film thickness, $\mu \mathrm{m}$ & \multicolumn{5}{|l|}{0.20} \\
\hline & Injection method (split, splitless, etc.) & \multicolumn{5}{|l|}{ 1:10 split } \\
\hline & Quant Method: ES = external standards (Y/N) & \multicolumn{5}{|l|}{$\mathrm{Y}$} \\
\hline & Number of ES used & \multicolumn{5}{|l|}{4.00} \\
\hline$\approx$ & Quant Method: IS = internal standards (Y/N) & \multicolumn{5}{|l|}{$\mathrm{Y}$} \\
\hline 全 & Number of IS used & \multicolumn{5}{|l|}{3.00} \\
\hline 莺 & IS used & \multicolumn{5}{|c|}{ C170, C190, C270 } \\
\hline$\sigma$ & IS added PRIOR to extraction of sample (Y/N) & \multicolumn{5}{|l|}{$\mathrm{Y}$} \\
\hline & Calibration model (linear, quad) & \multicolumn{5}{|c|}{ linear through zero } \\
\hline & Calibration range & \multicolumn{5}{|c|}{ blank to approx. $1600 \mathrm{mg} / \mathrm{L}$} \\
\hline 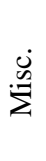 & Additional information, method reference & \multicolumn{5}{|c|}{$\begin{array}{l}\text { We spike our standards into BSA/NaCl ("fake" serum). Therefore, } \\
\text { density is not a variable as this "fake" serum has a density of } 1,02 \text {. Even } \\
\text { though the standards are already FAMEs, they are subjected to a } \\
\text { complete sample workup. }\end{array}$} \\
\hline
\end{tabular}


Table D-16: Method Descriptions for LC08-Total FA Method

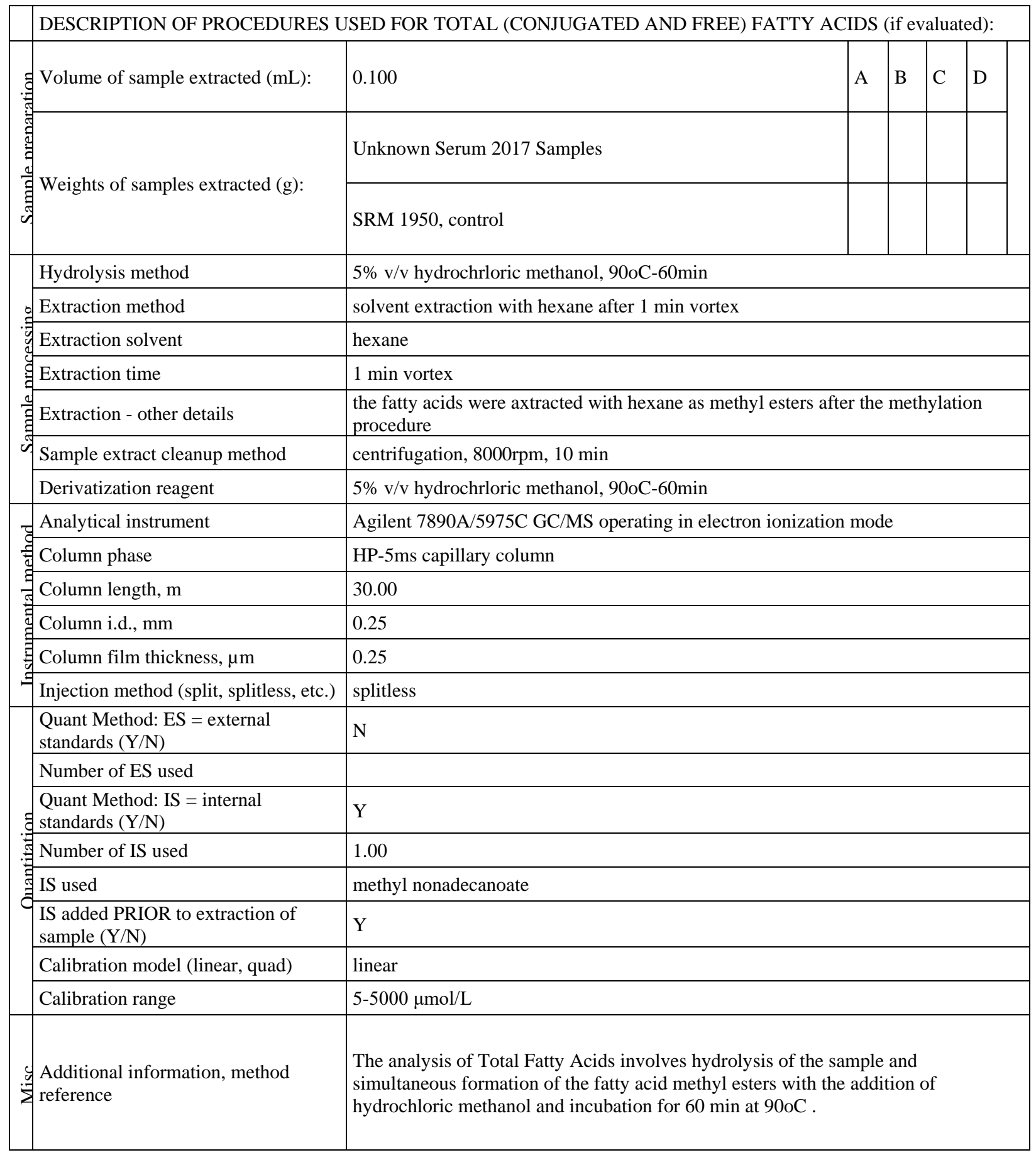


Table D-17: Method Descriptions for LC08-Free FA/FAME in Solutions Method

\begin{tabular}{|c|c|c|c|c|c|c|}
\hline \multirow{3}{*}{ 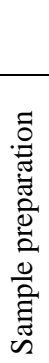 } & \multicolumn{6}{|c|}{ DESCRIPTION OF PROCEDURES USED FOR FREE FATTY ACID/FATTY ACID METHYL ESTER SOLUTIONS: } \\
\hline & Volume of sample extracted (mL): & 0.100 & $\begin{array}{l}\text { Unknown } \\
\text { Free FA Sol. } \\
\text { A }\end{array}$ & $\begin{array}{l}\text { Unknown } \\
\text { Free FA Sol. } \\
\text { B }\end{array}$ & $\begin{array}{l}\text { Unknown } \\
\text { Free FA Sol. } \\
\text { C }\end{array}$ & $\begin{array}{l}\text { Unknown } \\
\text { FAME/FA } \\
\text { Sol. D }\end{array}$ \\
\hline & Weights of samples extracted (g): & Solutions & & & & \\
\hline \multirow{7}{*}{ 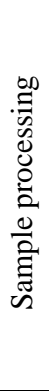 } & Hydrolysis method (if applies) & \multicolumn{5}{|c|}{$5 \% \mathrm{v} / \mathrm{v}$ hydrochrloric methanol, 90oC-60min } \\
\hline & Extraction method & \multicolumn{5}{|c|}{ solvent extraction with hexane after 1 min vortex } \\
\hline & Extraction solvent & \multicolumn{5}{|l|}{ hexane } \\
\hline & Extraction time & \multicolumn{5}{|c|}{1 min vortex } \\
\hline & Extraction - other details & \multicolumn{5}{|c|}{$\begin{array}{l}\text { the fatty acids were axtracted with hexane as methyl esters after the } \\
\text { methylation procedure }\end{array}$} \\
\hline & Sample extract cleanup method & \multicolumn{5}{|c|}{ centrifugation, 8000rpm, $10 \mathrm{~min}$} \\
\hline & Derivatization reagent & \multicolumn{5}{|c|}{$5 \% \mathrm{v} / \mathrm{v}$ hydrochrloric methanol, 90oC-60min } \\
\hline \multirow{6}{*}{ 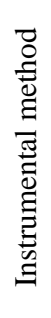 } & Analytical instrument & \multicolumn{5}{|c|}{ Agilent 7890A/5975C GC/MS operating in electron ionization mode } \\
\hline & Column phase & \multicolumn{5}{|c|}{ HP-5ms capillary column } \\
\hline & Column length, $\mathrm{m}$ & \multicolumn{5}{|l|}{30.00} \\
\hline & Column i.d., mm & \multicolumn{5}{|l|}{0.25} \\
\hline & Column film thickness, $\mu \mathrm{m}$ & \multicolumn{5}{|l|}{0.25} \\
\hline & Injection method (split, splitless, etc.) & \multicolumn{5}{|l|}{ splitless } \\
\hline \multirow{8}{*}{ 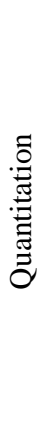 } & Quant Method: ES = external standards (Y/N) & \multicolumn{5}{|l|}{$\mathrm{N}$} \\
\hline & Number of ES used & & & & & \\
\hline & Quant Method: IS = internal standards $(\mathrm{Y} / \mathrm{N})$ & \multicolumn{5}{|l|}{ Y } \\
\hline & Number of IS used & \multicolumn{5}{|l|}{1.00} \\
\hline & IS used & \multicolumn{5}{|c|}{ methyl nonadecanoate } \\
\hline & IS added PRIOR to extraction of sample (Y/N) & \multicolumn{5}{|l|}{$\mathrm{Y}$} \\
\hline & Calibration model (linear, quad) & \multicolumn{5}{|l|}{ linear } \\
\hline & Calibration range & \multicolumn{5}{|c|}{$5-5000 \mu \mathrm{mol} / \mathrm{L}$} \\
\hline$\stackrel{\leftrightarrow}{\stackrel{n}{2}}$ & Additional information, method reference & \multicolumn{5}{|c|}{$\begin{array}{l}\text { We measured the free FA in the three solutions in the Fatty Acid methyl } \\
\text { ester form. }\end{array}$} \\
\hline
\end{tabular}


Table D-18: Method Descriptions for LC09-Total FA Method

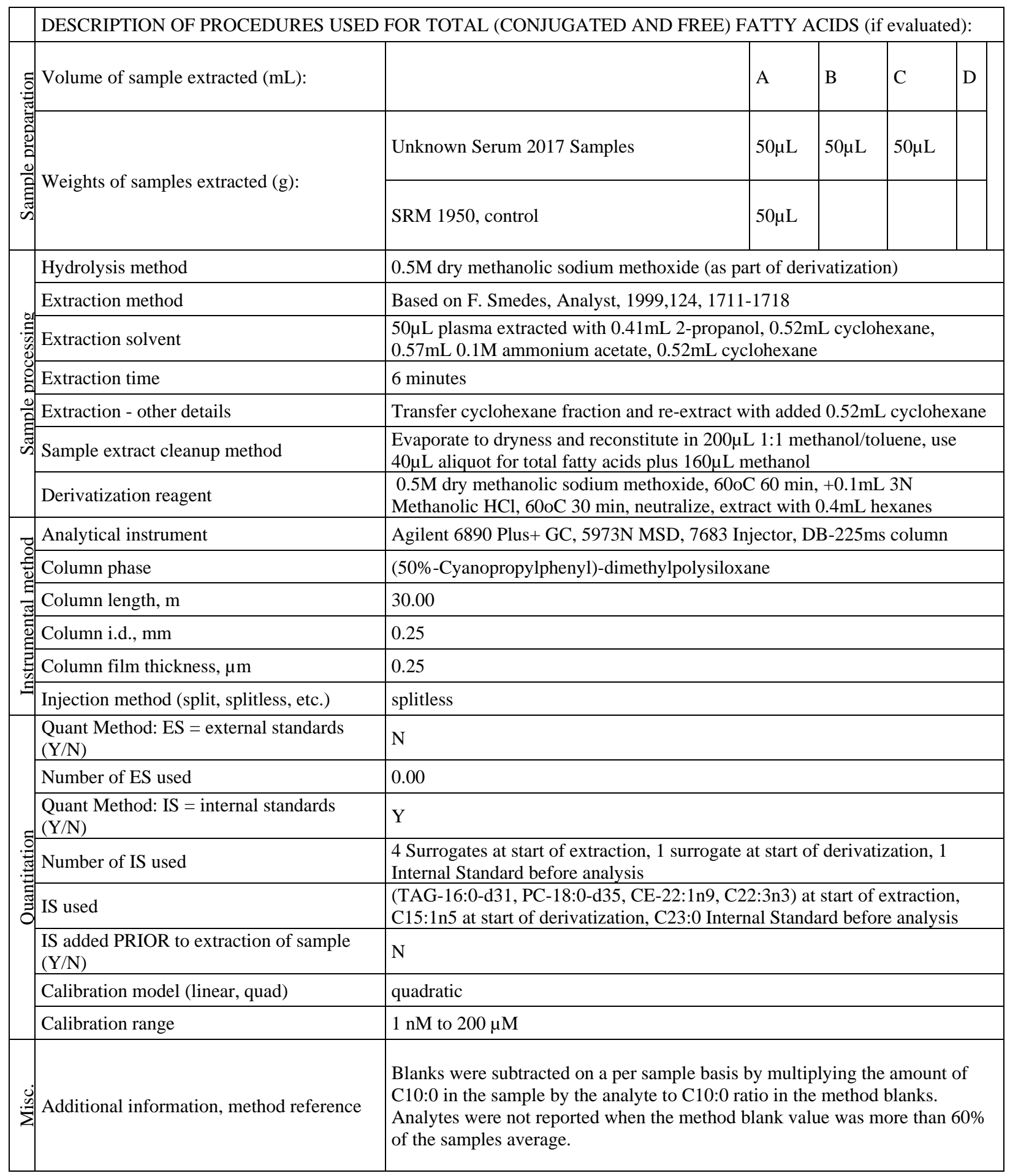


Table D-19: Method Descriptions for LC09-Free FA Method

\begin{tabular}{|c|c|c|c|c|c|c|}
\hline \multirow{4}{*}{ 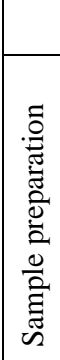 } & \multicolumn{6}{|c|}{ DESCRIPTION OF PROCEDURES USED FOR FREE FATTY ACIDS (if evaluated): } \\
\hline & Volume of sample extracted (mL): & & A & B & C & $\mathrm{D}$ \\
\hline & \multirow{2}{*}{ Weights of samples extracted (g): } & Unknown Serum 2017 Samples & $50 \mu \mathrm{L}$ & $50 \mu \mathrm{L}$ & $50 \mu \mathrm{L}$ & \\
\hline & & SRM 1950, control & $50 \mu \mathrm{L}$ & & & \\
\hline \multirow{7}{*}{ 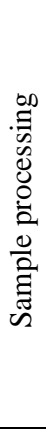 } & Hydrolysis method (if applies) & \multicolumn{5}{|l|}{ none } \\
\hline & Extraction method & \multicolumn{5}{|c|}{ Based on F. Smedes, Analyst, 1999,124, 1711-1718 } \\
\hline & Extraction solvent & \multicolumn{5}{|c|}{$\begin{array}{l}50 \mu \mathrm{L} \text { plasma extracted with } 0.41 \mathrm{~mL} \text { 2-propanol, } 0.52 \mathrm{~mL} \text { cyclohexane, } 0.57 \mathrm{~mL} \\
0.1 \mathrm{M} \text { ammonium acetate, } 0.52 \mathrm{~mL} \text { cyclohexane }\end{array}$} \\
\hline & Extraction time & \multicolumn{5}{|l|}{6 minutes } \\
\hline & Extraction - other details & \multicolumn{5}{|c|}{ Transfer cyclohexane fraction and re-extract with added $0.52 \mathrm{~mL}$ cyclohexane } \\
\hline & Sample extract cleanup method & \multicolumn{5}{|c|}{$\begin{array}{l}\text { Evaporate to dryness and reconstitute in } 200 \mu \mathrm{L} 1: 1 \text { methanol/toluene, use } \\
160 \mu \mathrm{L} \text { aliquot for total fatty acids plus } 120 \mu \mathrm{L} \text { methanol \& } 20 \mu \mathrm{L} \text { toluene }\end{array}$} \\
\hline & Derivatization reagent & \multicolumn{5}{|c|}{$\begin{array}{l}45 \mu \mathrm{L} \text { (trimethylsilyl)diazomethane (in nhexanes) for } 30 \text { minutes, evaporate to } \\
\text { dryness, reconstitute in } 200 \mu \mathrm{L} \text { hexanes }\end{array}$} \\
\hline \multirow{6}{*}{ 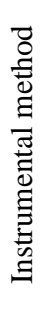 } & Analytical instrument & \multicolumn{5}{|c|}{ Agilent 6890 Plus+ GC, 5973N MSD, 7683 Injector, DB-225ms column } \\
\hline & Column phase & \multicolumn{5}{|c|}{ (50\%-Cyanopropylphenyl)-dimethylpolysiloxane } \\
\hline & Column length, m & \multicolumn{5}{|l|}{30.00} \\
\hline & Column i.d., mm & \multicolumn{5}{|l|}{0.25} \\
\hline & Column film thickness, $\mu \mathrm{m}$ & \multicolumn{5}{|l|}{0.25} \\
\hline & Injection method (split, splitless, etc.) & \multicolumn{5}{|l|}{ splitless } \\
\hline \multirow{8}{*}{ 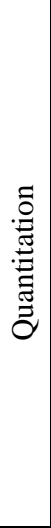 } & $\begin{array}{l}\text { Quant Method: ES = external standards } \\
\text { (Y/N) }\end{array}$ & \multicolumn{5}{|l|}{$\mathrm{N}$} \\
\hline & Number of ES used & \multicolumn{5}{|l|}{0.00} \\
\hline & $\begin{array}{l}\text { Quant Method: IS = internal standards } \\
(\mathrm{Y} / \mathrm{N})\end{array}$ & \multicolumn{5}{|l|}{$\mathrm{Y}$} \\
\hline & Number of IS used & \multicolumn{5}{|c|}{$\begin{array}{l}4 \text { Surrogates at start of extraction, } 1 \text { surrogate at start of derivatization, } 1 \\
\text { Internal Standard before analysis }\end{array}$} \\
\hline & IS used & \multicolumn{5}{|c|}{$\begin{array}{l}\text { (TAG-16:0-d31, PC-18:0-d35, CE-22:1n9, C22:3n3) at start of extraction, } \\
\text { C15:1n5 at start of derivatization, C23:0 Internal Standard before analysis }\end{array}$} \\
\hline & $\begin{array}{l}\text { IS added PRIOR to extraction of sample } \\
(\mathrm{Y} / \mathrm{N})\end{array}$ & \multicolumn{5}{|c|}{ 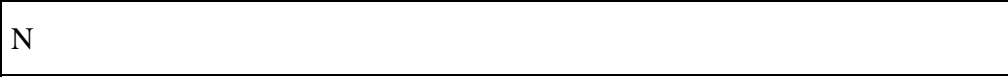 } \\
\hline & Calibration model (linear, quad) & \multicolumn{5}{|l|}{ quadratic } \\
\hline & Calibration range & \multicolumn{5}{|l|}{$1 \mathrm{nM}$ to $200 \mu \mathrm{M}$} \\
\hline ن. & Additional information, method reference & \multicolumn{5}{|c|}{$\begin{array}{l}\text { Blanks were subtracted on a per sample basis by multiplying the amount of } \\
\text { C10:0 in the sample by the analyte to C10:0 ratio in the method blanks. } \\
\text { Analytes were not reported when the method blank value was more than } 60 \% \\
\text { of the samples average. }\end{array}$} \\
\hline
\end{tabular}


Table D-20: Method Descriptions for LC09-Free FA/FAME in Solutions Method

\begin{tabular}{|c|c|c|c|c|c|c|}
\hline \multirow{3}{*}{ 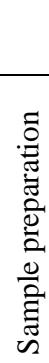 } & \multicolumn{6}{|c|}{ DESCRIPTION OF PROCEDURES USED FOR FREE FATTY ACID/FATTY ACID METHYL ESTER SOLUTIONS: } \\
\hline & Volume of sample extracted $(\mathrm{mL})$ : & & $\begin{array}{l}\text { Unknown } \\
\text { Free FA Sol. } \\
\text { A }\end{array}$ & $\begin{array}{l}\text { Unknown } \\
\text { Free FA Sol. } \\
\text { B }\end{array}$ & $\begin{array}{l}\text { Unknown } \\
\text { Free FA Sol. } \\
\text { C }\end{array}$ & $\begin{array}{l}\text { Unknown } \\
\text { FAME/FA } \\
\text { Sol. D }\end{array}$ \\
\hline & Weights of samples extracted (g): & Solutions & $40 \mu \mathrm{L}$ & $40 \mu \mathrm{L}$ & $40 \mu \mathrm{L}$ & $40 \mu \mathrm{L}$ \\
\hline \multirow{7}{*}{ 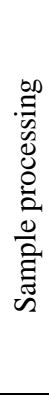 } & Hydrolysis method (if applies) & \multicolumn{5}{|l|}{ none } \\
\hline & Extraction method & \multicolumn{5}{|l|}{ none } \\
\hline & Extraction solvent & \multicolumn{5}{|l|}{ none } \\
\hline & Extraction time & \multicolumn{5}{|l|}{ N/A } \\
\hline & Extraction - other details & \multicolumn{5}{|l|}{ N/A } \\
\hline & Sample extract cleanup method & \multicolumn{5}{|c|}{ Dilute $40 \mu \mathrm{L}$ sample (in toluene) with $60 \mu \mathrm{L}$ toluene and $200 \mu \mathrm{L}$ methanol } \\
\hline & Derivatization reagent & \multicolumn{5}{|c|}{$\begin{array}{l}45 \mu \mathrm{L} \text { (trimethylsilyl)diazomethane (in nhexanes), derivatize for } 30 \text { minutes, } \\
\text { evaporate to dryness, reconstitute in } 200 \mu \mathrm{L} \text { hexanes }\end{array}$} \\
\hline \multirow{6}{*}{ 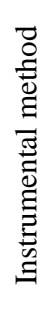 } & Analytical instrument & \multicolumn{5}{|c|}{ Agilent 6890 Plus+ GC, 5973N MSD, 7683 Injector, DB-225ms column } \\
\hline & Column phase & \multicolumn{5}{|c|}{ (50\%-Cyanopropylphenyl)-dimethylpolysiloxane } \\
\hline & Column length, $\mathrm{m}$ & \multicolumn{5}{|l|}{30.00} \\
\hline & Column i.d., mm & \multicolumn{5}{|l|}{0.25} \\
\hline & Column film thickness, $\mu \mathrm{m}$ & \multicolumn{5}{|l|}{0.25} \\
\hline & Injection method (split, splitless, etc.) & \multicolumn{5}{|l|}{ splitless } \\
\hline & $\begin{array}{l}\text { Quant Method: ES = external standards } \\
(\mathrm{Y} / \mathrm{N})\end{array}$ & \multicolumn{5}{|l|}{$\mathrm{N}$} \\
\hline & Number of ES used & \multicolumn{5}{|l|}{0.00} \\
\hline & $\begin{array}{l}\text { Quant Method: IS = internal standards } \\
(\mathrm{Y} / \mathrm{N})\end{array}$ & \multicolumn{5}{|l|}{$\mathrm{Y}$} \\
\hline 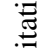 & Number of IS used & \multicolumn{5}{|c|}{5 Surrogates at start of derivatization, 1 Internal Standard before analysis } \\
\hline 䓌 & IS used & \multicolumn{5}{|c|}{$\begin{array}{l}\text { (TAG-16:0-d31, PC-18:0-d35, CE-22:1n9, C22:3n3 and C15:1n5) at start of } \\
\text { derivatization, C23:0 Internal Standard before analysis }\end{array}$} \\
\hline & $\begin{array}{l}\text { IS added PRIOR to extraction of sample } \\
(\mathrm{Y} / \mathrm{N})\end{array}$ & \multicolumn{5}{|c|}{ 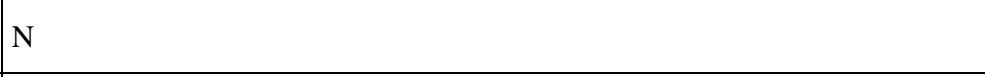 } \\
\hline & Calibration model (linear, quad) & \multicolumn{5}{|l|}{ quadratic } \\
\hline & Calibration range & \multicolumn{5}{|c|}{$1 \mathrm{nM}$ to $200 \mu \mathrm{M}$} \\
\hline 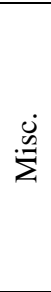 & Additional information, method reference & \multicolumn{5}{|c|}{$\begin{array}{l}\text { Blanks were subtracted on a per sample basis by multiplying the amount of } \\
\text { C10:0 in the sample by the analyte to C10:0 ratio in the method blanks. } \\
\text { Analytes were not reported when the method blank value was more than } 60 \% \\
\text { of the samples average. The values for C18:0, C18:1n7, and C18:1n9 were } \\
\text { above the highest standard in the calibration curve (144\%, } 142 \% \text {, and } 290 \% \\
\text { of the top standard respectively). All measurements were done as samples } \\
\text { derivatized to FAME's so FAME values could not be determined separately } \\
\text { from FAs in the samples. }\end{array}$} \\
\hline
\end{tabular}


Table D-21: Method Descriptions for LC10-Total FA Method

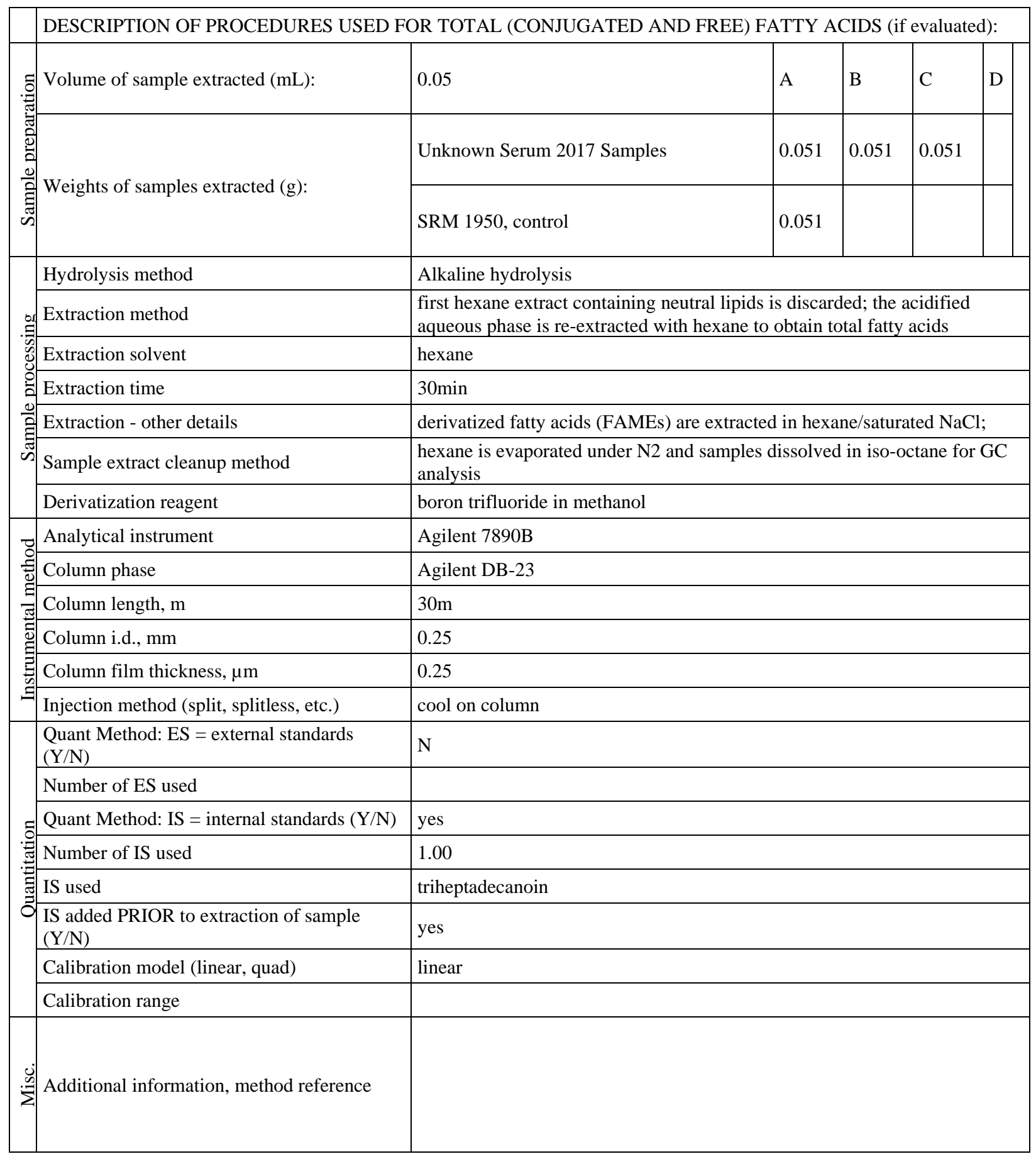


Table D-22: Method Descriptions for LC10-Free FA/FAME in Solutions Method

\begin{tabular}{|c|c|c|c|c|c|c|}
\hline & \multicolumn{6}{|c|}{ DESCRIPTION OF PROCEDURES USED FOR FREE FATTY ACID/FATTY ACID METHYL ESTER SOLUTIONS: } \\
\hline \multirow{2}{*}{ 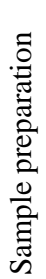 } & Volume of sample extracted $(\mathrm{mL})$ : & 0.05 & $\begin{array}{l}\text { Unknown } \\
\text { Free FA Sol. } \\
\text { A }\end{array}$ & $\begin{array}{l}\text { Unknown } \\
\text { Free FA Sol. } \\
\text { B }\end{array}$ & $\begin{array}{l}\text { Unknown } \\
\text { Free FA Sol. } \\
\text { C }\end{array}$ & $\begin{array}{l}\text { Unknown } \\
\text { FAME/FA } \\
\text { Sol. D }\end{array}$ \\
\hline & Weights of samples extracted (g): & Solutions & 0.04 & 0.04 & 0.04 & 0.04 \\
\hline \multirow{7}{*}{ 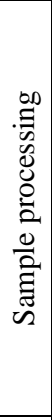 } & Hydrolysis method (if applies) & \multicolumn{5}{|c|}{ Alkaline hydrolysis } \\
\hline & Extraction method & \multicolumn{5}{|c|}{$\begin{array}{l}\text { first hexane extract containing neutral lipids is discarded; the acidified aqueous } \\
\text { phase is re-extracted with hexane to obtain total fatty acids }\end{array}$} \\
\hline & Extraction solvent & \multicolumn{5}{|l|}{ hexane } \\
\hline & Extraction time & \multicolumn{5}{|l|}{$30 \mathrm{~min}$} \\
\hline & Extraction - other details & \multicolumn{5}{|c|}{ derivatized fatty acids (FAMEs) are extracted in hexane/saturated $\mathrm{NaCl}$; } \\
\hline & Sample extract cleanup method & \multicolumn{5}{|c|}{$\begin{array}{l}\text { hexane is evaporated under N2 and samples dissolved in iso-octane for GC } \\
\text { analysis }\end{array}$} \\
\hline & Derivatization reagent & \multicolumn{5}{|c|}{ boron trifluoride in methanol } \\
\hline \multirow{6}{*}{ 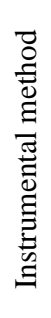 } & Analytical instrument & \multicolumn{5}{|c|}{ Agilent 7890B } \\
\hline & Column phase & \multicolumn{5}{|c|}{ Agilent DB-23 } \\
\hline & Column length, m & \multicolumn{5}{|l|}{$30 \mathrm{~m}$} \\
\hline & Column i.d., mm & \multicolumn{5}{|l|}{0.25} \\
\hline & Column film thickness, $\mu \mathrm{m}$ & \multicolumn{5}{|l|}{0.25} \\
\hline & Injection method (split, splitless, etc.) & \multicolumn{5}{|c|}{ cool on column } \\
\hline \multirow{8}{*}{ 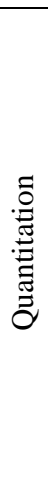 } & $\begin{array}{l}\text { Quant Method: ES = external standards } \\
(\mathrm{Y} / \mathrm{N})\end{array}$ & \multicolumn{5}{|l|}{$\mathrm{N}$} \\
\hline & Number of ES used & & & & & \\
\hline & $\begin{array}{l}\text { Quant Method: IS = internal standards } \\
\text { (Y/N) }\end{array}$ & \multicolumn{5}{|l|}{ yes } \\
\hline & Number of IS used & \multicolumn{5}{|l|}{1.00} \\
\hline & IS used & \multicolumn{5}{|c|}{ triheptadecanoin } \\
\hline & $\begin{array}{l}\begin{array}{l}\text { IS added PRIOR to extraction of sample } \\
(\mathrm{Y} / \mathrm{N})\end{array} \\
\end{array}$ & \multicolumn{5}{|l|}{ yes } \\
\hline & Calibration model (linear, quad) & \multicolumn{5}{|l|}{ linear } \\
\hline & Calibration range & \multicolumn{5}{|l|}{ 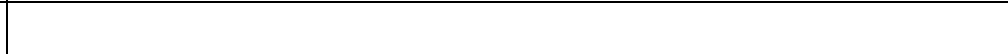 } \\
\hline 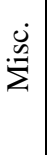 & $\begin{array}{l}\text { Additional information, method } \\
\text { reference }\end{array}$ & \\
\hline
\end{tabular}


Table D-23: Method Descriptions for LC11-Total FA Method

\begin{tabular}{|c|c|c|c|c|c|c|}
\hline & \multicolumn{6}{|c|}{ DESCRIPTION OF PROCEDURES USED FOR TOTAL (CONJUGATED AND FREE) FATTY ACIDS (if evaluated): } \\
\hline \multirow{3}{*}{ 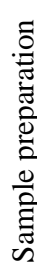 } & Volume of sample extracted (mL): & 0.20 & A & B & C & $\mathrm{D}$ \\
\hline & \multirow{2}{*}{ Weights of samples extracted (g): } & Unknown Serum 2017 Samples & 0.20 & 0.20 & 0.20 & \\
\hline & & SRM 1950, control & 0.20 & & & \\
\hline \multirow{7}{*}{ v } & Hydrolysis method & \multicolumn{5}{|l|}{ Liu et al 2010} \\
\hline & Extraction method & \multicolumn{5}{|l|}{ Solvent extraction } \\
\hline & Extraction solvent & \multicolumn{5}{|l|}{ Hexane:2-proponol (3:2, v/v) } \\
\hline & Extraction time & & & & & \\
\hline & Extraction - other details & & & & & \\
\hline & Sample extract cleanup method & \multicolumn{5}{|l|}{ Phase separartion and SPE catridge cleanup } \\
\hline & Derivatization reagent & & & & & \\
\hline \multirow{4}{*}{ 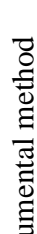 } & Analytical instrument & \multicolumn{5}{|l|}{ Thermo Trace GC-DSQ system } \\
\hline & Column phase & \multicolumn{5}{|c|}{$\begin{array}{l}\text { Rxi-5MS-coated 5\%diphenyl/95\%dimethyl polysiloxane capillary } \\
\text { column }\end{array}$} \\
\hline & Column length, $\mathrm{m}$ & \multicolumn{5}{|l|}{$1 / 30 / 1900$} \\
\hline & Column i.d., mm & \multicolumn{5}{|l|}{$1 / 0 / 1900$} \\
\hline \multirow[t]{2}{*}{$\underline{\Xi}$} & Column film thickness, $\mu \mathrm{m}$ & \multicolumn{5}{|l|}{0.25} \\
\hline & Injection method (split, splitless, etc.) & \multicolumn{5}{|l|}{ splitless } \\
\hline & Quant Method: ES = external standards (Y/N) & \multicolumn{5}{|l|}{$\mathrm{N}$} \\
\hline & Number of ES used & \multicolumn{5}{|l|}{ NA } \\
\hline$\approx$ & Quant Method: IS = internal standards (Y/N) & \multicolumn{5}{|l|}{ Y } \\
\hline 馬 & Number of IS used & \multicolumn{5}{|l|}{ one } \\
\hline สี & IS used & \multicolumn{5}{|l|}{ Methyl tridecanoate } \\
\hline 0 & IS added PRIOR to extraction of sample (Y/N) & \multicolumn{5}{|l|}{ Yes } \\
\hline & \multicolumn{6}{|l|}{ Calibration model (linear, quad) } \\
\hline \multicolumn{7}{|c|}{ Calibration range } \\
\hline$\sum^{2}$ & Additional information, method reference & \multicolumn{5}{|l|}{ Liu et al 2010} \\
\hline
\end{tabular}


Table D-24: Method Descriptions for LC11-Free FA/FAME in Solutions Method

\begin{tabular}{|c|c|c|c|c|c|c|}
\hline \multicolumn{7}{|c|}{ DESCRIPTION OF PROCEDURES USED FOR FREE FATTY ACID/FATTY ACID METHYL ESTER SOLUTIONS: } \\
\hline \multirow{2}{*}{ 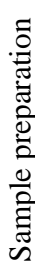 } & Volume of sample extracted $(\mathrm{mL})$ : & & $\begin{array}{l}\text { Unknown } \\
\text { Free FA Sol. } \\
\text { A }\end{array}$ & $\begin{array}{l}\text { Unknown } \\
\text { Free FA Sol. } \\
\text { B }\end{array}$ & $\begin{array}{l}\text { Unknown } \\
\text { Free FA Sol. } \\
\text { C }\end{array}$ & $\begin{array}{l}\text { Unknown } \\
\text { FAME/FA } \\
\text { Sol. D }\end{array}$ \\
\hline & Weights of samples extracted (g): & Solutions & 0.20 & 0.20 & 0.20 & $1 / 0 / 1900$ \\
\hline \multirow{7}{*}{ 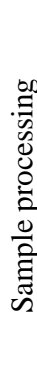 } & Hydrolysis method (if applies) & & & & & \\
\hline & Extraction method & \multicolumn{5}{|c|}{ Solvent extraction } \\
\hline & Extraction solvent & \multicolumn{5}{|c|}{ Hexane:2-proponol (3:2, v/v) } \\
\hline & Extraction time & & & & & \\
\hline & Extraction - other details & & & & & \\
\hline & Sample extract cleanup method & Phase sep & rartion and SPE & catridge cleant & & \\
\hline & Derivatization reagent & & & & & \\
\hline \multirow{6}{*}{ 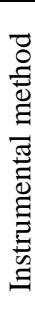 } & Analytical instrument & \multicolumn{5}{|c|}{ Thermo Trace GC-DSQ system } \\
\hline & Column phase & \multicolumn{5}{|c|}{ Rxi-5MS-coated 5\%diphenyl/95\%dimethyl polysiloxane capillary column } \\
\hline & Column length, $\mathrm{m}$ & \multicolumn{5}{|l|}{$1 / 30 / 1900$} \\
\hline & Column i.d., mm & \multicolumn{5}{|l|}{$1 / 0 / 1900$} \\
\hline & Column film thickness, $\mu \mathrm{m}$ & \multicolumn{5}{|l|}{0.25} \\
\hline & Injection method (split, splitless, etc.) & \multicolumn{5}{|l|}{ splitless } \\
\hline & $\begin{array}{l}\text { Quant Method: ES = external standards } \\
\text { (Y/N) }\end{array}$ & \multicolumn{5}{|l|}{$\mathrm{N}$} \\
\hline & Number of ES used & \multicolumn{5}{|l|}{ NA } \\
\hline$\approx$ & Quant Method: IS = internal standards $(\mathrm{Y} / \mathrm{N})$ & \multicolumn{5}{|l|}{$\mathrm{Y}$} \\
\hline 泀 & Number of IS used & \multicolumn{5}{|l|}{ one } \\
\hline 艺 & IS used & \multicolumn{5}{|c|}{ Methyl tridecanoate } \\
\hline$\sigma$ & $\begin{array}{l}\text { IS added PRIOR to extraction of sample } \\
(\mathrm{Y} / \mathrm{N})\end{array}$ & \multicolumn{5}{|l|}{ Yes } \\
\hline & \multicolumn{6}{|l|}{ Calibration model (linear, quad) } \\
\hline \multicolumn{7}{|c|}{ Calibration range } \\
\hline 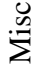 & Additional information, method reference & \multicolumn{5}{|c|}{ Liu et al 2010} \\
\hline
\end{tabular}


Table D-25: Method Descriptions for LC12-Total FA Method

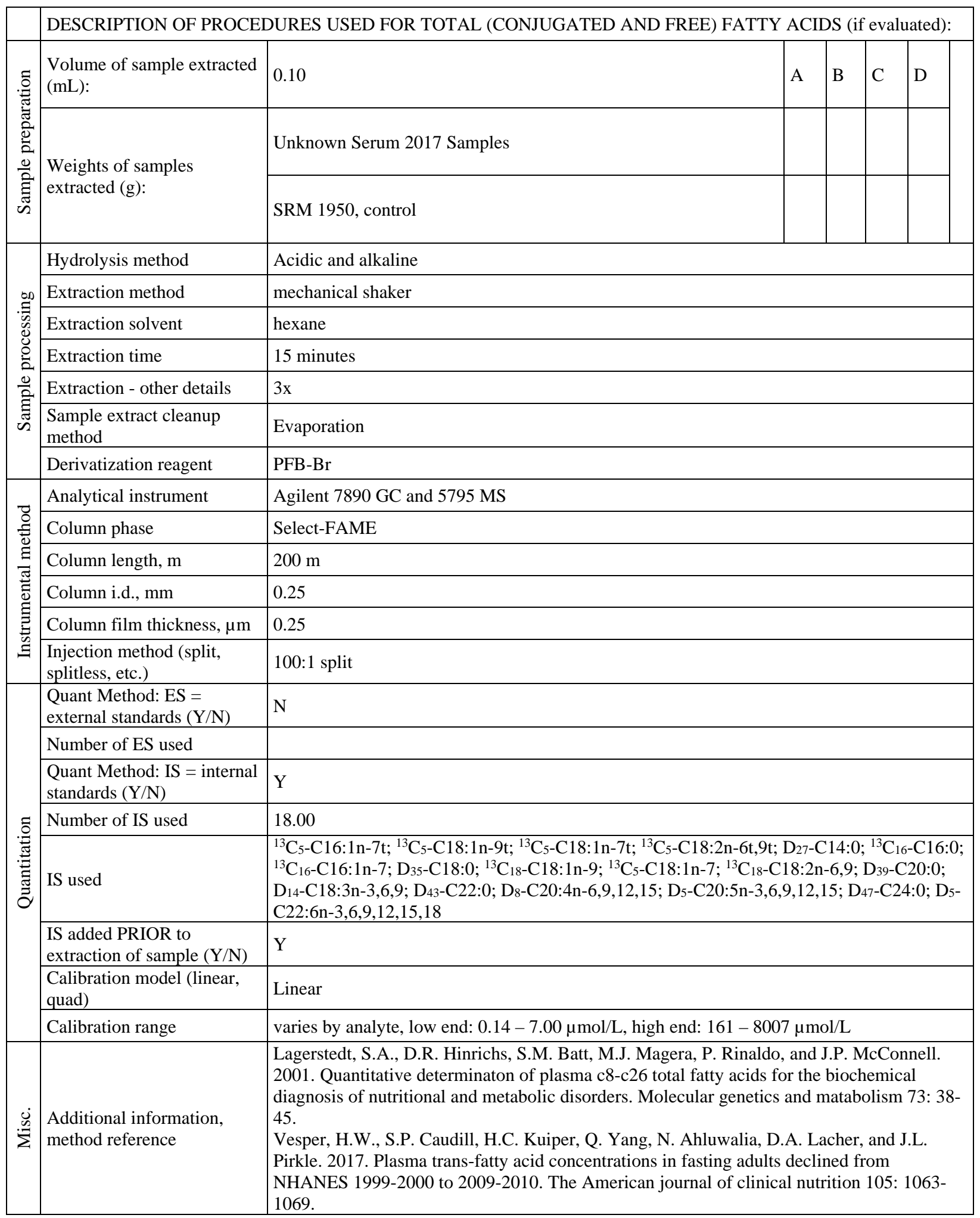


Table D-26: Method Description for LC12-Free FA/FAME in Solutions.

\begin{tabular}{|c|c|c|c|c|c|c|}
\hline \multirow{3}{*}{ 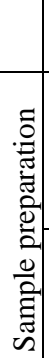 } & \multicolumn{6}{|c|}{ DESCRIPTION OF PROCEDURES USED FOR FREE FATTY ACID/FATTY ACID METHYL ESTER SOLUTIONS } \\
\hline & Volume of sample extracted (mL): & & $\begin{array}{l}\text { Unknown } \\
\text { Free FA Sol. } \\
\text { A }\end{array}$ & $\begin{array}{l}\text { Unknown } \\
\text { Free FA Sol. } \\
\text { B }\end{array}$ & $\begin{array}{l}\text { Unknown } \\
\text { Free FA Sol. } \\
\text { C }\end{array}$ & $\begin{array}{l}\text { Unknown } \\
\text { FAME/FA } \\
\text { Sol. D }\end{array}$ \\
\hline & Weights of samples extracted (g): & Solutions & & & & \\
\hline \multirow{7}{*}{ 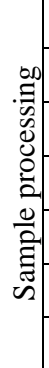 } & Hydrolysis method (if applies) & & & & & \\
\hline & Extraction method & & & & & \\
\hline & Extraction solvent & & & & & \\
\hline & Extraction time & & & & & \\
\hline & Extraction - other details & & & & & \\
\hline & Sample extract cleanup method & & & & & \\
\hline & Derivatization reagent & & & & & \\
\hline \multirow{6}{*}{ 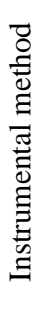 } & Analytical instrument & & & & & \\
\hline & Column phase & & & & & \\
\hline & Column length, $\mathrm{m}$ & & & & & \\
\hline & Column i.d., mm & & & & & \\
\hline & Column film thickness, $\mu \mathrm{m}$ & & & & & \\
\hline & Injection method (split, splitless, etc.) & & & & & \\
\hline \multirow{8}{*}{ 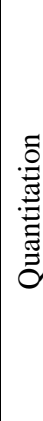 } & Quant Method: ES = external standards (Y/N) & & & & & \\
\hline & Number of ES used & & & & & \\
\hline & Quant Method: IS = internal standards (Y/N) & & & & & \\
\hline & Number of IS used & & & & & \\
\hline & IS used & & & & & \\
\hline & IS added PRIOR to extraction of sample (Y/N) & & & & & \\
\hline & Calibration model (linear, quad) & & & & & \\
\hline & Calibration range & & & & & \\
\hline 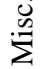 & Additional information, method reference & \multicolumn{5}{|c|}{ Same procedure as for total fatty acids } \\
\hline
\end{tabular}


Table D-27: Method Descriptions for LC13-Free Glycerol Method

\begin{tabular}{|c|c|c|c|c|c|c|}
\hline \multirow{4}{*}{ 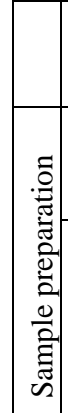 } & \multicolumn{6}{|c|}{ DESCRIPTION OF PROCEDURES USED FOR FREE GLYCEROL (if evaluated): } \\
\hline & Volume of sample extracted (mL): & $100 \mathrm{~mL}$ & A & B & C & $\mathrm{D}$ \\
\hline & \multirow{2}{*}{ Weights of samples extracted (g): } & Unknown Serum 2017 Samples & 0.00182 & 0.00164 & 0.00203 & \\
\hline & & SRM 1950, control & 0.05376 & & & \\
\hline \multirow{7}{*}{ 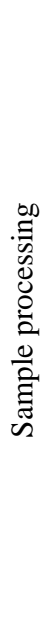 } & \multicolumn{6}{|l|}{ Hydrolysis method (if applies) } \\
\hline & Extraction method & \multicolumn{5}{|l|}{ 80v/v\% Ethanol precipitation } \\
\hline & Extraction solvent & \multicolumn{5}{|l|}{ Ethanol } \\
\hline & Extraction time & \multicolumn{5}{|l|}{30 minutes } \\
\hline & Extraction - other details & \multicolumn{5}{|c|}{$\begin{array}{l}\text { 200uL of samples were admixed } 1600 \mathrm{uL} \text { ethanol contained with } \\
200 \mathrm{uL} \text { of the internal standard solution using an automatic pipettor. } \\
\text { The mixtures were vortexed and stand for } 30 \mathrm{~min} \text { at room temperature } \\
\text { followed by centrifugation ( } 3000 \mathrm{~g} \text { x } 15 \mathrm{~min}) \text {. }\end{array}$} \\
\hline & Sample extract cleanup method & \multicolumn{5}{|c|}{$\begin{array}{l}\text { The supernatant of ethanol precipitations were derivatized with a } 3: 1 \\
\text { mixture of pyridine and acetic anhydride at } 70{ }^{\circ} \mathrm{C} \text { for } 60 \mathrm{~min} \text { at the } \\
\text { heating block. } \\
\text { And the derivatized samples were treated by water, hydrogen chloride } \\
(6 \mathrm{~N}) \text { and Sodium bicarbonate splution ( } 8 \% \text { ) to remove excess pyridine, } \\
\text { acetic anhydride and by-product acetic acid. }\end{array}$} \\
\hline & Derivatization reagent & \multicolumn{5}{|c|}{ 3:1 mixture of pyridine and acetic anhydride } \\
\hline \multirow{6}{*}{ 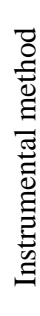 } & Analytical instrument & \multicolumn{5}{|c|}{ the quadrupole mass spectrometer JMS-Q1050GC (JEOL Ltd.) } \\
\hline & Column phase & \multicolumn{5}{|c|}{ DB17-ms (50\% phenyl) - methyl-polysiloxane } \\
\hline & Column length, m & \multicolumn{5}{|l|}{$30 \mathrm{~m}$} \\
\hline & Column i.d., mm & \multicolumn{5}{|l|}{0.25} \\
\hline & Column film thickness, $\mu \mathrm{m}$ & \multicolumn{5}{|l|}{0.25} \\
\hline & Injection method (split, splitless, etc.) & \multicolumn{5}{|l|}{ splitless } \\
\hline \multirow{8}{*}{ 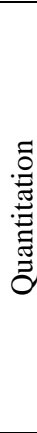 } & Quant Method: ES = external standards $(\mathrm{Y} / \mathrm{N})$ & \multicolumn{5}{|l|}{$\mathrm{N}$} \\
\hline & \multicolumn{6}{|l|}{ Number of ES used } \\
\hline & Quant Method: IS = internal standards (Y/N) & \multicolumn{5}{|l|}{$\mathrm{Y}$} \\
\hline & Number of IS used & \multicolumn{5}{|l|}{1.00} \\
\hline & IS used & \multicolumn{5}{|l|}{ 13C2-glycerol } \\
\hline & IS added PRIOR to extraction of sample (Y/N) & \multicolumn{5}{|l|}{$\mathrm{Y}$} \\
\hline & Calibration model (linear, quad) & \multicolumn{5}{|l|}{ linear } \\
\hline & \multicolumn{6}{|l|}{ Calibration range } \\
\hline 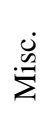 & Additional information, method reference & \multicolumn{5}{|c|}{$\begin{array}{l}\text { Clinical chemistry 58(4) 768-776 } 2012 \\
\text { Reference Measurement Procedure for Total Glycerides by Isotope } \\
\text { Dilution GC-MS }\end{array}$} \\
\hline
\end{tabular}


Table D-28: Method Descriptions for LC13-Total Glycerides Method

\begin{tabular}{|c|c|c|c|c|c|c|}
\hline \multirow{4}{*}{ 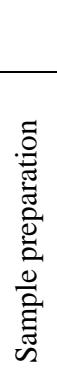 } & \multicolumn{6}{|c|}{ DESCRIPTION OF PROCEDURES USED FOR TOTAL GLYCERIDES (if evaluated): } \\
\hline & Volume of sample extracted (mL): & $100 \mathrm{~mL}$ & A & B & $\mathrm{C}$ & $\mathrm{D}$ \\
\hline & \multirow{2}{*}{ Weights of samples extracted (g): } & Unknown Serum 2017 Samples & 1.541 & 1.547 & 1.545 & \\
\hline & & SRM 1950, control & 1.097 & & & \\
\hline \multirow{7}{*}{ 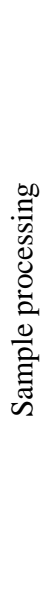 } & Hydrolysis method (if applies) & \multicolumn{5}{|c|}{ 0.3mol/L KOH in ethanol , 60 degree $\mathrm{C}, 60$ minutes } \\
\hline & Extraction method & \\
\hline & Extraction solvent & \multirow{3}{*}{\multicolumn{5}{|c|}{$\begin{array}{l}\text { Two hundred microliter of samples that have been contained with } \\
\text { 200uL of } 1,3-13 \mathrm{C} 2 \text {-glycerol internel standard solution were } \\
\text { hydrolyzed in } 0.30 \mathrm{~mol} / \mathrm{L} \text { alcoholic } \mathrm{KOH} \text { at } 60^{\circ} \mathrm{C} \text { for } 1 \mathrm{~h} \text { followed } \\
\text { by the drying under nitrogen using the Turbovap LV evaporator. }\end{array}$}} \\
\hline & Extraction time & & & & & \\
\hline & Extraction - other details & & & & & \\
\hline & Sample extract cleanup method & \multicolumn{5}{|c|}{$\begin{array}{l}\text { The dried residues were derivatized with a } 3: 1 \text { mixture of pyridine } \\
\text { and acetic anhydride at } 70{ }^{\circ} \mathrm{C} \text { for } 60 \mathrm{~min} \text { at the heating block. } \\
\text { And the derivatized samples were treated by water, hydrogen } \\
\text { chloride }(6 \mathrm{~N}) \text { and Sodium bicarbonate splution ( } 8 \% \text { ) to remove } \\
\text { excess pyridine, acetic anhydride and by-product acetic acid. }\end{array}$} \\
\hline & Derivatization reagent & \multicolumn{5}{|c|}{ 3:1 mixture of pyridine and acetic anhydride } \\
\hline \multirow{6}{*}{ 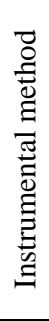 } & Analytical instrument & \multicolumn{5}{|c|}{ the quadrupole mass spectrometer JMS-Q1050GC (JEOL Ltd.) } \\
\hline & Column phase & \multicolumn{5}{|c|}{ DB17-ms (50\% phenyl) - methyl-polysiloxane } \\
\hline & Column length, m & \multicolumn{5}{|l|}{$30 \mathrm{~m}$} \\
\hline & Column i.d., mm & \multicolumn{5}{|l|}{0.25} \\
\hline & Column film thickness, $\mu \mathrm{m}$ & \multicolumn{5}{|l|}{0.25} \\
\hline & Injection method (split, splitless, etc.) & \multicolumn{5}{|l|}{ splitless } \\
\hline \multirow{8}{*}{ 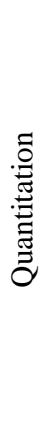 } & Quant Method: ES = external standards (Y/N) & \multicolumn{5}{|l|}{$\mathrm{N}$} \\
\hline & Number of ES used & & & & & \\
\hline & Quant Method: IS = internal standards (Y/N) & \multicolumn{5}{|l|}{$\mathrm{Y}$} \\
\hline & Number of IS used & \multicolumn{5}{|l|}{1.00} \\
\hline & IS used & \multicolumn{5}{|l|}{ 13C2-glycerol } \\
\hline & IS added PRIOR to extraction of sample (Y/N) & \multicolumn{5}{|l|}{$\mathrm{Y}$} \\
\hline & Calibration model (linear, quad) & \multicolumn{5}{|l|}{ linear } \\
\hline & Calibration range & \\
\hline 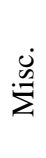 & Additional information, method reference & \multicolumn{5}{|c|}{$\begin{array}{l}\text { Reference : } \\
\text { Clinical chemistry 58(4) 768-776 } 2012 \\
\text { Reference Measurement Procedure for Total Glycerides by Isotope } \\
\text { Dilution GC-MS }\end{array}$} \\
\hline
\end{tabular}


Table D-29: Method Descriptions for LC14-Total FA Method

\begin{tabular}{|c|c|c|c|c|c|c|}
\hline & \multicolumn{6}{|c|}{ DESCRIPTION OF PROCEDURES USED FOR TOTAL (CONJUGATED AND FREE) FATTY ACIDS (if evaluated): } \\
\hline \multirow{3}{*}{ 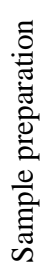 } & Volume of sample extracted (mL): & & A & B & C & $\mathrm{D}$ \\
\hline & \multirow{2}{*}{ Weights of samples extracted (g): } & Unknown Serum 2017 Samples & 0.10 & 0.10 & 0.10 & \\
\hline & & SRM 1950, control & 0.10 & & & \\
\hline \multirow{7}{*}{ 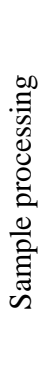 } & Hydrolysis method & \multicolumn{5}{|l|}{ Acid/Base } \\
\hline & Extraction method & \multicolumn{5}{|c|}{$\begin{array}{l}\text { Liquid Phase Extraction usuing automated liquid handler } \\
\text { (Hamilton) }\end{array}$} \\
\hline & Extraction solvent & \multicolumn{5}{|c|}{ Hexane } \\
\hline & Extraction time & \multicolumn{5}{|l|}{$\sim 1.5 \mathrm{hrs}$} \\
\hline & Extraction - other details & & & & & \\
\hline & Sample extract cleanup method & & & & & \\
\hline & Derivatization reagent & Pentafluorobenzyl Bromide (PF & & & & \\
\hline \multirow{6}{*}{ 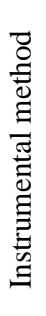 } & Analytical instrument & \multicolumn{5}{|c|}{ Agilent 7890A GC oven + 5975C MS } \\
\hline & Column phase & \multicolumn{5}{|l|}{ TG-Polar } \\
\hline & Column length, $\mathrm{m}$ & \multicolumn{5}{|l|}{ 2/29/1900 } \\
\hline & Column i.d., mm & \multicolumn{5}{|l|}{0.25} \\
\hline & Column film thickness, $\mu \mathrm{m}$ & \multicolumn{5}{|l|}{0.20} \\
\hline & Injection method (split, splitless, etc.) & \multicolumn{5}{|l|}{ split } \\
\hline \multirow{8}{*}{ 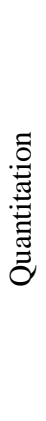 } & Quant Method: ES = external standards (Y/N) & \multicolumn{5}{|l|}{$\mathrm{N}$} \\
\hline & \multicolumn{6}{|l|}{ Number of ES used } \\
\hline & Quant Method: IS = internal standards $(\mathrm{Y} / \mathrm{N})$ & \multicolumn{5}{|l|}{$\mathrm{Y}$} \\
\hline & Number of IS used & \multicolumn{5}{|l|}{18.00} \\
\hline & IS used & & & & & \\
\hline & IS added PRIOR to extraction of sample $(\mathrm{Y} / \mathrm{N})$ & \multicolumn{5}{|l|}{$\mathrm{Y}$} \\
\hline & Calibration model (linear, quad) & \multicolumn{5}{|l|}{ analyte dependant } \\
\hline & Calibration range & & & & & \\
\hline 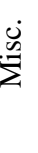 & \multicolumn{6}{|l|}{ Additional information, method reference } \\
\hline
\end{tabular}


Table D-30: Method Descriptions for LC14-Free FA/FAME in Solutions Method

\begin{tabular}{|c|c|c|c|c|c|c|}
\hline \multirow{3}{*}{ 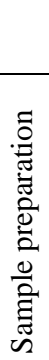 } & \multicolumn{6}{|c|}{ DESCRIPTION OF PROCEDURES USED FOR FREE FATTY ACID/FATTY ACID METHYL ESTER SOLUTION } \\
\hline & Volume of sample extracted (mL): & & $\begin{array}{l}\text { Unknown } \\
\text { Free FA Sol. } \\
\text { A }\end{array}$ & $\begin{array}{l}\text { Unknown } \\
\text { Free FA Sol. } \\
\text { B }\end{array}$ & $\begin{array}{l}\text { Unknown } \\
\text { Free FA Sol. } \\
\text { C }\end{array}$ & $\begin{array}{l}\text { Unknown } \\
\text { FAME/FA } \\
\text { Sol. D }\end{array}$ \\
\hline & Weights of samples extracted (g): & Solutions & 0.08045 & 0.07918 & 0.07904 & 0.07968 \\
\hline \multirow{7}{*}{ 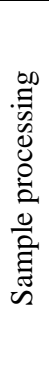 } & Hydrolysis method (if applies) & \multicolumn{5}{|l|}{ Acid/Base } \\
\hline & Extraction method & \multicolumn{5}{|c|}{ Liquid Phase Extraction usuing automated liquid handler (Hamilton) } \\
\hline & Extraction solvent & \multicolumn{5}{|l|}{ Hexane } \\
\hline & Extraction time & \multicolumn{5}{|l|}{$\sim 1.5 \mathrm{hrs}$} \\
\hline & Extraction - other details & & & & & \\
\hline & Sample extract cleanup method & & & & & \\
\hline & Derivatization reagent & \multicolumn{5}{|c|}{ Pentafluorobenzyl Bromide (PFBBr) } \\
\hline \multirow{6}{*}{ 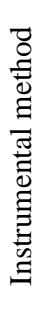 } & Analytical instrument & \multicolumn{5}{|c|}{ Agilent 7890A GC oven + 5975C MS } \\
\hline & Column phase & \multicolumn{5}{|l|}{ TG-Polar } \\
\hline & Column length, $\mathrm{m}$ & \multicolumn{5}{|l|}{ 2/29/1900 } \\
\hline & Column i.d., mm & \multicolumn{5}{|l|}{0.25} \\
\hline & Column film thickness, $\mu \mathrm{m}$ & \multicolumn{5}{|l|}{0.20} \\
\hline & Injection method (split, splitless, etc.) & \multicolumn{5}{|l|}{ split } \\
\hline \multirow{8}{*}{ 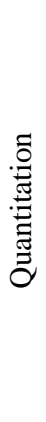 } & Quant Method: ES = external standards (Y/N) & \multicolumn{5}{|l|}{$\mathrm{N}$} \\
\hline & Number of ES used & \\
\hline & Quant Method: IS = internal standards $(\mathrm{Y} / \mathrm{N})$ & \multicolumn{5}{|l|}{$\mathrm{Y}$} \\
\hline & Number of IS used & \multicolumn{5}{|l|}{18.00} \\
\hline & IS used & \\
\hline & IS added PRIOR to extraction of sample (Y/N) & \multicolumn{5}{|l|}{$\mathrm{Y}$} \\
\hline & Calibration model (linear, quad) & \multicolumn{5}{|c|}{ analyte dependant } \\
\hline & Calibration range & \multicolumn{5}{|c|}{ 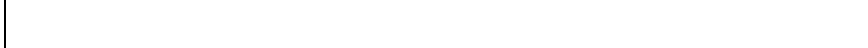 } \\
\hline$\sum_{\substack{0 \\
\sum}}^{ن}$ & Additional information, method reference & \\
\hline
\end{tabular}

